\title{
Intramembrane Signal Transduction and Cell Envelope Stress Response
} in Bacillus subtilis

\author{
Dissertation \\ zur Erlangung des Doktorgrades \\ der Mathematisch-Naturwissenschaftlichen Fakultäten \\ der Georg-August-Universität zu Göttingen
}

\author{
vorgelegt von \\ Sina Jordan \\ aus Essen
}

Göttingen 2007 
D 7

Referent: Prof. Dr. Jörg Stülke

Korreferent: PD Dr. Michael Hoppert

Tag der mündlichen Prüfung: 01.11.2007 


\section{Danksagung}

Der grösste Dank gilt natürlich meinem Doktorvater Dr. Thorsten Mascher! Also Thorsten, an dieser Stelle nochmal vielen Dank für das interessante Thema, die umfassende Betreuung und das supernette Arbeitsklima. Ich hab mich in Deinem Labor sehr wohl gefühlt und hatte eine Menge Spass! Du hast mich immer unterstützt und mir das Gefühl gegeben, gute Arbeit zu leisten. Es war schön, in einem so motivierten Umfeld arbeiten zu dürfen!

Ein herzliches Dankeschön geht an Prof. Dr. Jörg Stülke für die Übernahme des Referates und die Möglichkeit, in seiner Abteilung arbeiten zu dürfen.

Für die Übernahme des Korreferats danke ich PD Dr. Michael Hoppert.

Ausserdem möchte ich mich bei Dr. Leendert Hamoen für die Möglichkeit bedanken, in seiner Arbeitsgruppe in Newcastle am Fluoreszenzmikroskop arbeiten zu dürfen.

Ein besonderer Dank geht natürlich auch an Bärbel Herbst für die Bereitstellung diverser Arbeitsmaterialien. Du hast mir das Arbeiten im Labor sehr erleichtert!

Den Mitarbeitern der GenoMIK möchte ich für die Durchführung unzähliger Sequenzierungen danken.

Ich habe das Glück gehabt, mit sehr vielen unwahrscheinlich netten Kollegen arbeiten zu dürfen. Da steht an erster Stelle natürlich Falk Kalamorz! Falk, ich danke Dir ganz herzlich für die schöne gemeinsame Zeit während meiner Doktorarbeit. Dabei möchte ich mich ganz besonders für die hunderte perfekt gekochten Kaffees bedanken, ohne die ich wahrscheinlich nicht überlebt hätte. Auch die (fast) täglichen eMails während meiner Zeit in Newcastle haben mir sehr geholfen, nicht an Einsamkeit zu sterben. Deshalb ein dickes Danke für alle lebensrettenden Taten während der letzten 3 Jahre!!!

Nun zum "Girls Camp": Während ihres GP II und der anschliessenden Diplomarbeit sind mir Tina Wecke, Eva Rietkötter, Diana Hoyer und Anja Junker gute Freundinnen gewesen. Ausserdem sind Ihre Ergebnisse zum Teil mit in diese Arbeit eingeflossen.

Tina, wo soll ich da anfangen? Wir konnten über nahezu alles reden, und das haben wir ja auch getan. Angefangen bei der gemeinsamen Leidenschaft für Pferde, über Beziehungen bis hin zu ausgedehnten Lästereien. Mir hat diese Zeit viel Spass gemacht, ich werde das sehr vermissen!

Eva, auch wir haben uns super verstanden, Du warst mir ein würdiger Gegner ;-) . Zu jeder Zeit einen trockenen Spruch auf den Lippen, das ist schon bemerkenswert. Nicht zu vergessen Deine herausragende Leistung am Kickertisch! 
Diana, ich habe die Zusammenarbeit mit Dir wirklich genossen. Schon seit Deinem GP II haben wir uns gut verstanden und ich bedanke mich sehr für die nette Zusammenarbeit! Ausserdem wünsche ich Dir und dem Baby alles Gute und viel Erfolg bei Deiner Doktorarbeit!

Anja, wir hatten eine lustige Zeit und sehr viel Spass im Labor. Auch den Cocktailabend werde ich nicht vergessen! Also hier von einer Zicke zur anderen: Danke für die schöne Zeit! Bastian Dörrbecker, Maria Martinec und Karen Schrecke haben während ihres GP II Ergebnisse erziehlt, die mit in diese Arbeit eingeflossen sind. Dafür vielen Dank!

Basti, es hat immer sehr viel Spass gemacht, mit Dir zu arbeiten, zu reden oder zu kickern. Ich wünsche Dir, dass Du bei Deiner Doktorarbeit so nette Kollegen findest wie ich!

Auch bei Claudine Hames und Sebastian Hübner möchte ich mich bedanken. Ich bin gerne mit Euch essen gegangen, hatte Spass beim pokern und war froh über die Schlafgelegenheit, die Ihr mir immer angeboten habt. Lasst Euch nicht unterkriegen, auch Ihr habt es auch bald geschafft!

Ganz lieben Dank ins ferne Newcastle an Sven Halbedel, der sich dort gut um mich gekümmert und seine Bench mit mir geteilt hat.

Auch allen anderen Mitgliedern der Abteilung Danke für die schöne Zeit, auch wenn ihr nicht namentlich erwähnt worden seid.

Ohne meine Eltern hätte ich das alles natürlich nie geschafft. Danke, dass Ihr mich wieder für einige Zeit bei Euch aufgenommen und mir immer den Rücken gestärkt habt! Für die ständige Unterstützung, die Betreuung von Sheila und die tausend anderen Dinge, die Ihr für mich tut, bin ich Euch wirklich sehr dankbar!

Meinem Mann Michael möchte ich ganz besonders danken. Michi, Du stehst immer hinter mir, hilfst mir, gibst mir Kraft, bestärkst mich in meinen Entscheidungen (auch wenn diese uns räumlich trennen), tröstest mich und tust noch tausend andere Dinge, die Dich zum wichtigsten Menschen in meinem Leben machen, und das nun schon seit 13 Jahren. Ich liebe Dich über alles! 


\section{Content}

Content I

List of abbrevations II

List of publications

Summary 1

Zusammenfassung 2

1. Introduction

A. Cell-envelope stress response in Bacillus subtilis and other Firmicutes bacteria $\quad 3$

B. Aims of this work $\quad 28$

2. Regulation of LiaRS-dependent gene expression in Bacillus subtilis:

Identification of inhibitor proteins, regulator binding sites and target genes of a conserved cell envelope stress-sensing two-component system

3. LiaRS-dependent gene expression is embedded in transition state regulation in Bacillus subtilis

4. Discussion

5. References

6. Supplementary material 


\section{List of abbreviations}

AAA+ $\quad$ ATPase associated with various cellular activities

ABC ATP binding cassette

B. Bacillus

Bac bacitracin

bp base pair

CAMP cationic antimicrobial peptide

cAMP cyclic adenosine monophosphate

CES cell envelope stress

CESR cell envelope stress response

cGMP cyclic guanosine monophosphate

$\mathrm{cm} \quad$ chloramphenicol (resistance cassette)

DNA deoxyribonucleic acid

E. Escherichia

ECF extracytoplasmic function

e.g. for example (exempli gratia)

Fig. figure

FMN flavine mononucleotide

GAF cyclic GMP, adenylyl cyclase, FhlA

GFP green fluorescent protein

h hour

HAMP histidine kinase, adenylyl cyclase, methyl-accepting chemotaxisprotein, phosphatase

HK histidine kinase

i. e. $\quad$ that is (it est)

IMHK intramembrane-sensing histidine kinase

LB Luria Bertani

min. minute

MLS Erythromycin/Lincomycin (resistance cassette)

$\mathrm{NAD}(\mathrm{P}) \mathrm{H}$ nicotinamide adenine dinucleotide phosphate, reduced form

ONPG 2-nitrophenyl- $\beta$-D-galactopyranoside

PBP penicillin-binding protein

PCR polymerase chain reaction 
pmf proton motiv force

RR response regulator

RT reverse transcription

SD Shine-Dalgarno (sequence)

sec. $\quad$ seconds

spec spectinomycin (resistance cassette)

TA teichoic acid

Tab. table

TCS two-component system

tet tetracyclin (resistance cassette)

TMR transmembrane region

UDP uridine 5'-diphosphate

wt wildtype

X-Gal 5-Bromo-4-chloro-3-indolyl- $\beta$-D-galactopyranoside 


\section{List of publications}

Jordan, S., Junker, A., Helmann, J. D., and Mascher, T. 2006. Regulation of LiaRSdependent gene expression in Bacillus subtilis: Identification of inhibitor proteins, regulator binding sites and target genes of a conserved cell envelope stress-sensing twocomponent system. Journal ofBacteriology 188: 5153-5166

Jordan, S., Rietkötter, E., Strauch, M. A., Kalamorz, F., Butcher, B. G., Helmann, J. D., and Mascher, T. 2007. LiaRS-dependent gene expression is embedded in transition state regulation in Bacillus subtilis. Microbiology 153: 2530-2540

Jordan, S., Hutchings, M. I., and Mascher, T. Cell envelope stress response in Grampositive bacteria. FEMS Microbiology Reviews: submitted 


\section{Summary}

The bacterial cell envelope is the first and major line of defence against threats from the environment. It is an essential and vulnerable structure that gives the cell its shape and counteracts the high internal osmotic pressure. It also provides an important sensory interface and molecular sieve, mediating both information flow and controlled transport of solutes. The cell envelope is also the target for numerous antibiotics. Therefore, the monitoring and maintaining of cell envelope integrity in the presence of envelope perturbating agents and conditions is crucial for survival. In Bacillus subtilis a complex regulatory network, consisting of 7 signal transducing systems, orchestrates the cell envelope stress response. Two forms of regulatory systems can be found: ECF- $\sigma$ factors and two component systems (TCS). One of these TCS is the LiaRS system that responds to cell wall antibiotics that interfere with the undecaprenol cycle and to perturbation of the cytoplasmic membrane. It is encoded by the last two genes of the liaIHGFSR locus. Without cell envelope stress, the last 4 genes are constitutively expressed from a weak promoter upstream of liaG to ensure the presence of the two-component system in the cell.

The activated response regulator LiaR induces the expression of the lia operon. LiaR binds to a palindromic sequence with a 7-4-7 inverted repeat, which is located $75 \mathrm{bp}$ upstream of the liaI start codon. In addition to the liaI promoter $\left(\mathrm{P}_{\text {lial }}\right)$, the promoter of the $y h c Y Z-y h d A$ operon and of the $y d h E$ gene could be identified as target promoters of LiaR in B. subtilis.

A systematic deletion analysis of the lia operon revealed that LiaF is a negative regulator of LiaR-dependent gene expression: a non-polar liaF deletion leads to constitutive activation of all three characterized LiaR-dependent promoters. The liaF gene is conserved in both sequence and genomic context in the Firmicutes group of Gram-positive bacteria, located directly upstream of liaSR orthologs. Therefore, LiaF together with LiaRS forms a threecomponent system.

In the transition to stationary phase, $\mathrm{P}_{\text {lial }}$ is induced without exogenous stimuli. This induction is LiaR-dependent and additionally regulated by AbrB and Spo0A. During logarithmic growth the transition state regulator AbrB binds to the liaI promoter and prevents expression of the lia operon. Spo0A regulates $\mathrm{P}_{\text {lial }}$ induction in transition state indirectly by repressing the expression of AbrB. 
Die bakterielle Zellhülle verleiht dem Zellkörper Form und Stabilität, wirkt dem starken osmotischen Innendruck entgegen und stellt eine Permeabilitätsbarriere da. Sie wirkt als molekulares Sieb und bietet der Zelle als äußerste Schicht Schutz gegen schädliche Umwelteinflüsse. Außerdem ist die Zellhülle aber auch die Verbindung zwischen einem Bakterium und seiner Umwelt. Viele Antibiotika greifen die Zellhülle oder ihre Synthese an. Deshalb ist ein ständiges Überwachen der Zellhüllintegrität für ein Bakterium lebenswichtig. Die Zellhüllstress-Antwort in Bacillus subtilis wird durch sieben signaltransduzierende Systeme vermittelt, zu denen drei ECF- $\sigma$-Faktoren und vier Zweikomponentensysteme gehören. Eines dieser Zweikomponentensysteme ist das LiaRS-System, welches auf Zellwandantibiotika, die den Lipid II-Zyklus hemmen, und auf Zerstörung der Cytoplasmamembran reagiert. Es wird von den letzten beiden Genen des liaIHGFSR Lokus kodiert. Ohne Zellhüllstress werden die letzten vier Gene des Operons von einem schwachen konstitutiven Promotors vor liaG exprimiert, wodurch das Vorhandensein des Zweikomponentensystems in der Zelle sichergestellt wird.

Der aktivierte Response Regulator LiaR induziert die Expression des lia Operons, indem er an eine palindromische Sequenz mit einem 7-4-7 Motiv, die sich 75 bp vor dem lial-Startkodon befindet, bindet. Zusätzlich zum liaI Promotor $\left(\mathrm{P}_{\text {lial }}\right)$ konnten die Promotoren des yhcYZ-yhdA Operon und des $y d h E$ Gens als Zielpromotoren von LiaR in B. subtilis identifiziert werden.

Eine systematische Deletionsanalyse des lia Operons zeigte, dass LiaF als negativer Regulator der LiaR-abhängigen Genexpression fungiert: eine nicht-polare Deletion von liaF führte zu konstitutiver Aktivierung aller drei charakterisierter LiaR-abhängigen Promotoren. Sowohl Sequenz als auch genomischer Kontext des liaF-Gens sind in der Gruppe der Firmicuten konserviert, wobei liaF stets stromaufwärts der liaSR-Orthologen lokalisiert ist. Daher bildet LiaF zusammen mit LiaRS ein Dreikomponenten-System.

Während des Übergangs zur stationären Wachstumsphase (transition state) wird $\mathrm{P}_{\text {lial }}$ ohne exogene Stimuli induziert. Diese Induktion ist LiaR-abhängig und wird zusätzlich von den Proteinen AbrB und Spo0A reguliert. Während der logarhythmischen Wachstumsphase ist der transition state Regulator $\mathrm{AbrB}$ an den liaI Promotor gebunden und verhindert so die Expression des lia Operons. Spo0A reguliert die Induktion von $\mathrm{P}_{\text {liaI }}$ indirekt, indem es die Expression von $\mathrm{AbrB}$ verhindert. 


\section{Chapter 1:}

\section{Introduction}

A. Cell Envelope Stress Response in Bacillus subtilis and Other Firmicutes Bacteria

This chapter is an excerpt of the following publication:

Jordan, S., Hutchings, M. I., and Mascher, T. Cell envelope stress response in Grampositive bacteria. FEMS Microbiology Reviews: submitted

Author contributions:

Sina Jordan performed the comparative genomic analysis and draw the figures. The review was written by Sina Jordan, Matthew I. Hutchings and Thorsten Mascher. 


\section{Introduction}

Life in the microbial world is characterized by fierce competition, nutritional hardship, and often life-threatening changes of external (i.e. physicochemical) parameters. Adaptive responses of a bacterium to its environment are therefore one defining cornerstone of microbial life in its natural context, irrespective of the individual life style or habitat. Such adaptations require the sensitive monitoring of numerous environmental parameters (input) to orchestrate the activity of intricate and complex regulatory systems that initiate or re-adjust adequate cellular responses (output) in a continuous balancing act between costs and gain. No surprise then that we find efficient stress response systems - aimed to maintain the functionality and integrity of the cell under all circumstances - embedded in the genomic blueprint of almost any bacterium studied to date (Storz and Hengge-Aronis, 2000).

The Gram-positive cell envelope. One of the crucial cellular structures is the cell envelope and its integrity has to be ensured, at all times and any costs. A detailed description of the biosynthesis and chemical composition of the Gram-positive cell envelope is beyond the scope of this review, and readers are referred to a number of excellent reviews on this topic (Archibald et al., 1993; Delcour et al., 1999; Foster and Popham, 2002). Suffice it to say, the Gram-positive cell envelope only consists of two functional layers (compared to three in Gram-negative bacteria) that enclose the cellular contents: a cytoplasmic membrane, surrounded by a thick cell wall. It lacks an outer membrane (and therefore a periplasmic space sensu stricto, see below). The Gram-positive peptidoglycan sacculus - in contrast to its single-layered Gram-negative counterpart - is a three-dimensional multi-layered net-like structure of about $50 \mathrm{~nm}$ thickness that can withstand high turgor pressures (up to $20 \mathrm{~atm}$, i.e. more than a racing bike tire!). Due to the combination of rigid sugar chains perpendicularly crosslinked with flexible peptide bridges, the mesh of this net is a strong, but also elastic stress-bearing structure (Delcour et al., 1999; Höltje, 1998). It is a highly dynamic supermolecule that undergoes permanent biosynthesis, assembly, maturation, disassembly, and recycling, to allow maintenance of cell shape, cellular growth and division at the same time (Archibald et al., 1993).

The Gram-positive peptidoglycan sacculus is interspersed with almost equal amounts of teichoic acids (TAs) that can either be tethered to the membrane (lipoteichoic acids) or covalently linked to the sugar backbone of the peptidoglycan sacculus (wall TAs) (Delcour et al., 1999; Foster and Popham, 2002). TAs are important components of the Gram-positive 
cell wall, and play a crucial role in defining the physicochemical properties of the envelope: They are poly-anionic, phosphate-rich linear polymers mainly responsible for the overall negative net charge of the Gram-positive cell surface (Bhavsar et al., 2004). TAs - as peptidoglycan - were generally viewed as essential biopolymers of Gram-positive bacteria. But recent studies in both Staphylococcus aureus and Bacillus subtilis - while clearly verifying the fundamental role of TAs for the overall cell integrity and fitness (Weidenmaier et al., 2004; Weidenmaier et al., 2005) - challenge this assumption by demonstrating the dispensability of wall TAs, at least under laboratory conditions (D'Elia et al., 2006a; D'Elia et $a l .$, 2006b). In contrast, recent work on lipoteichoic acid biosynthesis in S. aureus clearly indicates the essentiality of this biopolymer (Gründling and Schneewind, 2007).

In addition to these basic features of a Gram-positive cell wall, a number of additional cell envelope structures are present in many bacteria, and often play an important role for virulence, antibiotic resistance, colonization, and multicellular differentiation. These structures include extracellular polysaccharide capsules and biofilm matrices (Branda et al., 2005; Miyake and Iijima, 2004; O'Riordan and Lee, 2004), as well as proteinaceous S-layers (Schäffer and Messner, 2005; Sleytr et al., 2007). Moreover, the cell walls of mycobacteria and corynebacteria show a unique architecture that includes additional layers, consisting of arabinogalactans and mycolic acid, that surround the cell wall and renders these pathogens impenetrable for many antibiotics (Dover et al., 2004).

Many central questions on the mechanism and control of cell envelope homeostasis including aspects of its biosynthesis (such as the Lipid II cycle), turnover and overall architecture - still remain largely unanswered, despite its fundamental cellular role and decades of research. The three-dimensional architecture of the sacculus is a matter of an ongoing (and inspiring) debate between two competing - and mutually exclusive - models: peptidoglycan sheets versus scaffolds (Dmitriev et al., 2005; Vollmer and Höltje, 2004). Another area of controversy is the presence of a periplasmic space, which is generally viewed as a hallmark feature restricted to Gram-negative bacteria. Contradictory to that, recent advances in electron microscopic techniques indicated the presence of a periplasmic-like space of about $20 \mathrm{~nm}$ width in Gram-positive bacteria, located between the membrane and the cell wall (Matias and Beveridge, 2005, 2006). These findings emphasize the limitations in our understanding of the Gram-positive cell envelope architecture.

The cell wall - in addition to its roles as a shape-giving structure, an exoskeleton (to protect the cell from its environment and counteract the turgor pressure), and molecular sieve - is also discussed as a potent endotoxin in bacterial sepsis (Myhre et al., 2006). Moreover, the 
envelope also acts as a diffusion barrier (allowing and necessitating selective transport), and a communication interface (mediating information exchange) between the cell and its surrounding. The latter functions are primarily provided by the cytoplasmic membrane and its embedded proteins.

Antibiotics targeting the cell envelope. Because of its many crucial functions, the cell envelope is a prime target for numerous antibiotics, including many with high clinical relevance, that interfere with virtually any step in its biosynthesis (Koch, 2003; Lazar and Walker, 2002; Silver, 2003, 2006; Walsh, 2003). Only some of the more common cell wall antibiotics important in the context of this review, as well as their respective major bacterial resistance mechanisms, will be introduced very briefly in the following paragraphs. Their site of interference with cell wall biosynthesis is schematically summarized in Fig. 1.1. All cell wall antibiotics either directly inhibit the enzymatic activity mediating a cell wall reaction (shown in red in Fig. 1.1), or sequester the substrate of a given step (indicated in green in Fig. 1.1) (Silver, 2006).

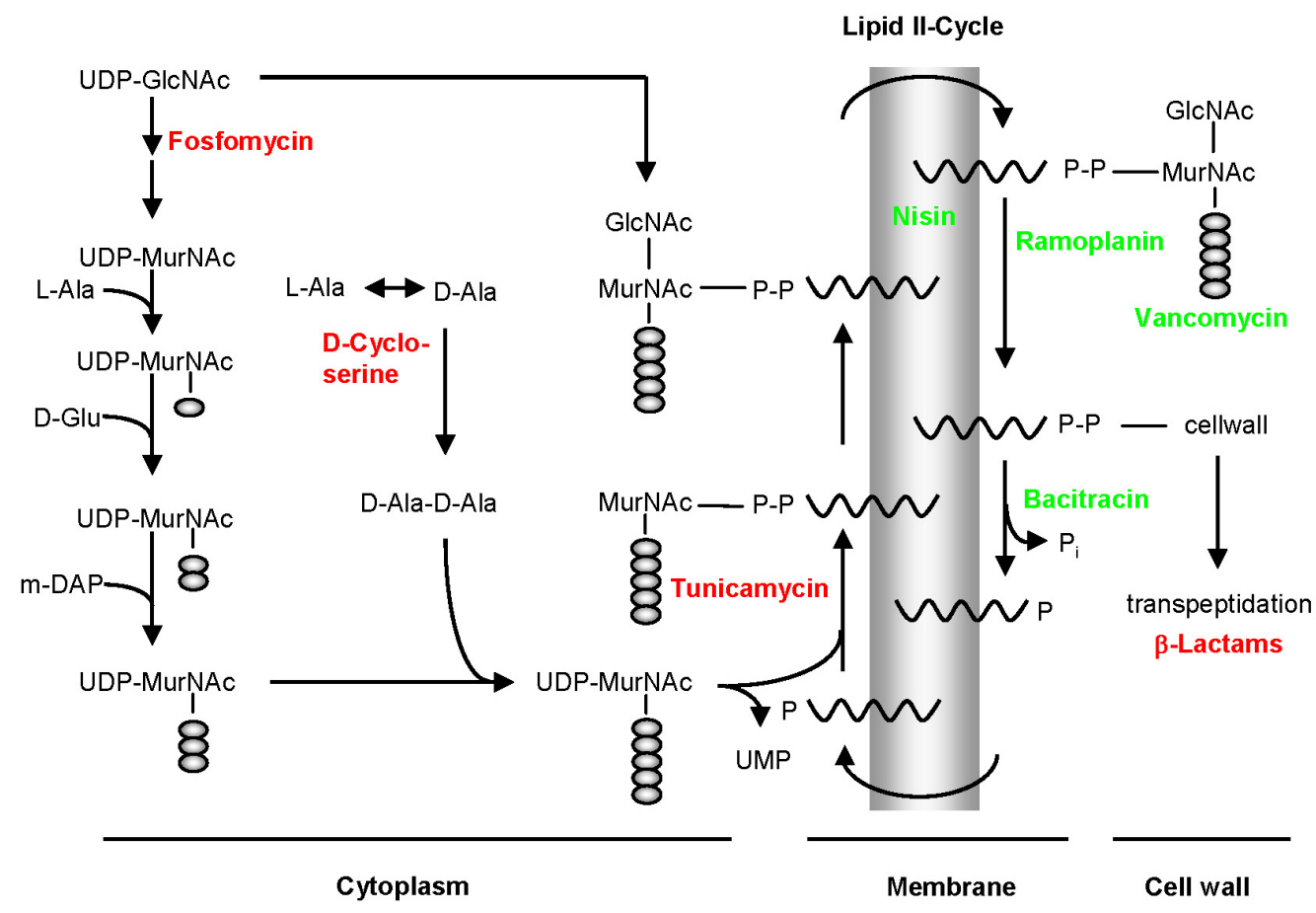

Figure 1.1. Cell wall biosynthesis and its inhibition by antibiotics. Important steps in cell wall biosynthesis are schematically depicted, and their cellular location is indicated below. Some cell wall antibiotics relevant for this review are given and placed next to the step they inhibit. Antibiotics in green sequester the substrate of the given step; those in red inhibit the corresponding enzyme. See text for details on their action. This figure was originally based in parts on (Silver, 2003), with modifications. 
Fosfomycin inhibits the first step committed step, the formation of UDP-N-acetyl-muramic acid from UDP-N-acetyl-glucosamine. It functions as an inactivating structural analogue of phosphoenol pyruvate, the co-substrate of the MurA-catalyzed reaction (Kahan et al., 1974). Resistance either arises by spontaneous mutations in the transport pathways (Horii et al., 1999), or is conferred by glutathione/metallothiol transferases that enzymatically inactivate fosfomycin (Bernat et al., 1997; Cao et al., 2001; Suarez and Mendoza, 1991).

D-cycloserine prevents the completion of the pentapetide side chain, the canonical crosslinking agent of the peptidoglycan network. It inhibits both the D-alanine racemase, which converts L-alanine to D-alanine, and the D-alanine/D-alanine ligase, which catalyzes the formation of the corresponding dipeptide (Lambert and Neuhaus, 1972; Neuhaus and Lynch, 1964). Resistance can either be achieved by overexpression of the target proteins or by an efflux pump (Feng and Barletta, 2003; Matsuo et al., 2003).

A number of antibiotics, including lantibiotics, ramoplanin, vancomycin, or bacitracin, interfere with the Lipid II cycle, the bottleneck of cell wall biosynthesis (Breukink and de Kruijff, 2006). Lipid II is the basic peptidoglycan building block, N-acetyl-glucosamine/Nacetyl muramic acid-pentapeptide covalently linked to the lipid carrier undecaprenol through a pyrophosphate ester bridge (Delcour et al., 1999).

Lantibiotics (such as nisin) are polycyclic peptide-derived antimicrobial agents that are ribosomally synthesized and posttranslationally modified to their active forms. They contain the unusual amino acid lanthionine as their name-giving feature and "highjack" Lipid II as a docking molecule to form pores, ultimately resulting in cell lysis (Breukink and de Kruijff, 2006; Chatterjee et al., 2005). The primary resistance mechanism against these positively charged peptide antibiotics is lowering of the overall negative net charge of the Gram-positive cell wall by $\mathrm{D}$-alanine incorporation into the poly-anionic TAs. Some cases of enzymatic degradation or modification of the lantibiotic have also been reported (Breukink and de Kruijff, 2006).

The overall positive charge and pore formation as the mode of antimicrobial action are shared by the wider family of so-called cationic antimicrobial peptides (CAMPs), which represent an important defense mechanism of the human immune system and have gained a lot of attention in recent years due to their potential as future therapeutics (Brogden, 2005; Giuliani et al., 2007; Hancock and Sahl, 2006).

Ramoplanin, a non-ribosomally synthesized lipoglycodepsipeptide antibiotic (Walker et al., 2005), inhibits the transglycosylation step of peptidoglycan biosynthesis by binding Lipid II 
at the extracellular surface of the cytoplasmic membrane (Hu et al., 2003). No resistance has been reported, so far (Breukink and de Kruijff, 2006).

Vancomycin and other glycopeptide antibiotics, such as teicoplanin, block glycan polymerization and cross-linking by tightly binding to the D-alanyl-D-alanine dipeptide terminus of Lipid II and nascent peptidoglycan (Kahne et al., 2005). Resistance is gained by reprogramming cell wall biosynthesis, incorporating alternative peptide termini, such as Dalanyl-D-lactate, instead of D-Ala-D-Ala (Healy et al., 2000; Walsh et al., 1996).

Bacitracin is a cyclic non-ribosomally synthesized dodecylpeptide antibiotic that requires the coordination of a divalent metal ion for its biological activity (Ming and Epperson, 2002). It binds very tightly to undecaprenyl pyrophosphate, thereby preventing the recycling of the lipid carrier by dephosphorylation (Stone and Strominger, 1971; Storm and Strominger, 1973). Four different mechanisms of bacitracin resistance have been described so far: (1) expression of bacitracin-specific ABC transporters (Mascher et al., 2003; Ohki et al., 2003a; Podlesek et al., 1995), (2) de novo synthesis of undecaprenyl phosphate (Cain et al., 1993; Chalker et al., 2000), (3) expression of alternative undecaprenyl pyrophosphate phosphatases (Bernard et al., 2005; Cao and Helmann, 2002; Ohki et al., 2003b), or (4) exopolysaccharide production (Pollock et al., 1994; Tsuda et al., 2002).

Penicillin and other $\beta$-lactams covalently modify the active site of transpeptidases (which are therefore called penicillin-binding proteins, or PBPs), by mimicking the D-alanyl-D-alanine terminus of the pentapeptide side chain (Strominger and Tipper, 1965). Resistance can be achieved by one of three known mechanisms (Poole, 2004; Wilke et al., 2005): (1) biosynthesis of $\beta$-lactamases that inactivate the antibiotic (Ghuysen, 1991), (2) expression of mutated $p b p$ alleles, so-called mosaic genes, encoding low-affinity PBPs that maintain their physiological function, but show a decreased $\beta$-lactam binding (Dowson et al., 1994; Hakenbeck, 1999), or (3) removal of the antibiotic from its site of action by $\beta$-lactam-specific efflux pumps (Poole, 2005).

The majority of classical antibiotics are produced by microorganisms of the soil biosphere (Berdy, 2005), such as bacilli (Butcher and Helmann, 2006; Stein, 2005), fungi (Anke, 1997), and most notably the actinomycetes (Champness, 2000; McNeil and Brown, 1994) and presumably to inhibit the growth of competitors. Stress responses and the development of counter strategies, including efficient resistance mechanisms, are therefore widespread survival strategies amongst soil bacteria to succeed in this habitat (D'Costa et al., 2006). Likewise, pathogenic bacteria encounter antimicrobials as part of the host's defense system, which also lead to the evolution of adequate stress responses and resistance mechanisms for 
survival. Only very recently, evolutionarily speaking, were pathogenic bacteria suddenly also challenged with antibiotics from the soil biosphere, in the form of novel 'magic bullets' for clinical antimicrobial therapy that were initially thought to eradicate the problem of lifethreatening bacterial infections once and for all. As we know now, antibiotic-resistant bacteria emerged faster than novel antimicrobials can be developed and approved for clinical use (Vicente et al., 2006). The evolution of novel traits of antibiotic resistance mechanisms in human pathogens and their commensal microbial brethren, through a combination of spontaneous beneficial mutations and horizontal gene transfer, happened at breathtaking speed, and sometimes included the transfer of whole functional modules consisting of sensitive antibiotic detection systems together with their respective target genes mediating resistance. This is most notably illustrated by the spread of vancomycin resistance amongst clinical isolates of enterococci and staphylococci (Palumbi, 2001; Walsh and Howe, 2002). Such traits, in the context of cell wall antibiotics, are one central aspect of bacterial CESR.

\section{Cell envelope stress (response)}

The physiological role of the cell envelope in combination with the presence of agents and/or conditions that can alter or even destroy this essential cellular structure necessitate that its integrity is closely monitored. The corresponding signal transducing regulatory systems respond to alterations and dysfunctions of the envelope and induce appropriate countermeasures to repair damage and secure functionality.

Before giving a comprehensive overview on this stress response and the regulatory systems mediating it, we first need to define the term. In contrast to "intuitive" stresses such as heat or osmotic shock, it is not easy to put a finger on cell envelope stress. Obviously, many stress conditions, including those mentioned above, affect the integrity of the cell envelope one way or another, without being referred to as cell envelope stress. The Gram-negative definition "Sensing and responding to damaged proteins in the extracytoplasmic compartments, collectively known as the cell envelope." (Ruiz and Silhavy, 2005) - is neither applicable nor helpful, due to the fundamental differences in cell envelope architecture between Gramnegative and -positive bacteria. At present, the best definition we can offer is derived from the approach by which CESRs and most of the regulatory systems involved have been identified and studied.

With very few exceptions, the model signaling systems orchestrating the Gram-positive envelope stress response were initially identified by one of three approaches. (1) They turned out to be responsible for an antibiotic resistance phenotype in (spontaneous) mutants, as 
exemplified by the CiaRH system of Streptococcus pneumoniae (Guenzi et al., 1994). (2) They were identified in the course of global gene expression (DNA microarray) studies to characterize the response of an organism when challenge with a cell wall antibiotic. This approach was used to decipher the complex regulatory network orchestrating CESR in the Gram-positive model bacterium B. subtilis (Cao et al., 2002b; Mascher et al., 2003; Pietiäinen et al., 2005). (3) Their potential role was identified during phenotyping of systematic mutational libraries to elucidate the role of signal transducing systems in a given organism (Hancock and Perego, 2004).

For this review, we will use the term CESR for Gram-positive bacteria based on the following definition: "The cell envelope stress response of a Gram-positive bacterium consists of those signal transducing regulatory systems (and their regulons) that are involved in sensing and responding to the presence of cell wall antibiotics and other envelope perturbating conditions."

Obviously, such a narrow definition is prone to generating blind spots. Some of the resulting gaps can be closed by comparative approaches, based on the overlap between different stress responses. We are aware of the potential pitfalls of such a definition. We therefore offer it as a suggestion to be evaluated, challenged and subsequently improved by alternative experimental approaches. The analysis of conditional lethal or overexpressing mutants in cell wall biosynthesis genes by transcriptomics, as exemplified by recent work on the cell wall stress stimulon of $S$. aureus (McAleese et al., 2006; Sobral et al., 2007) is one very promising example of such an alternative approach that harbors great potential towards that goal.

\section{Regulatory principles orchestrating CESR in Gram-positive and -negative bacteria.}

While the architecture of the cell envelope - and therefore the definition of the corresponding stress - differs greatly between Gram-positive and -negative bacteria, the regulatory principles orchestrating the corresponding stress responses are remarkably similar. CESR in the Gram-negative model organism Escherichia coli and related bacteria is well investigated and has been reviewed recently (Raivio, 2005; Rowley et al., 2006; Ruiz and Silhavy, 2005). It is orchestrated by one alternative $\sigma$ factor, three TCS and the phage shock protein response. For in-depth information on these systems, readers are referred to the cited review articles.

The essential extracytoplasmic function (ECF) $\sigma$ factor $\sigma^{\mathrm{E}}$, has been intensively studied in $E$. coli. It is induced in the presence of misfolded (outer membrane) proteins in the periplasm which can accumulate during growth at elevated temperatures (Ades, 2004; Alba and Gross, 2004; Rowley et al., 2006). It controls a large regulon including proteins that act directly on 
misfolded periplasmic proteins or are involved in the synthesis of lipopolysacchrides (Rhodius et al., 2006).

The CpxAR TCS is activated by elevated external $\mathrm{pH}$, misfolded periplasmic proteins or changes in the lipid composition of the inner membrane (Ruiz and Silhavy, 2005). It is subject to a negative feedback regulation exerted by the periplasmic protein CpxP (Buelow and Raivio, 2005; Fleischer et al., 2007). Its regulon consists of more than 100 proteins, and partly overlaps with that of $\sigma^{\mathrm{E}}$ (De Wulf et al., 2002).

The RcsBC TCS, together with the unstable auxiliary regulator RcsA, represents a complex phosphorelay system that is involved in regulating capsule biosynthesis, biofilm formation, and the expression of additional periplasmic and membrane proteins (Majdalani and Gottesman, 2005).

Little is known about BaeRS, the third envelope stress-sensing TCS of E. coli. It protects the cell from perturbations of the envelope caused by the presence of indole or misfolded proteins, acting in conjunction with the Cpx system (Raffa and Raivio, 2002). Furthermore, it regulates the expression of a multidrug-efflux pump, thereby conferring resistance to antimicrobial compounds, including $\beta$-lactam antibiotics (Hirakawa et al., 2003; Nagakubo et al., 2002).

The physiological role of the PspA-mediated phage-shock protein response is less well understood. It is induced by various stress conditions such as filamentous phage infection (hence the name), heat shock, osmotic shock, exposure to organic solvents and proton ionophores as well as long incubation under alkaline conditions. Strains defective in the Psp system show only minor physiological aberrations, for instance poor stationary phase survival, increased motility, slower protein secretion, and some defects in maintaining the membrane potential (i.e. proton motif force) when stressed (Darwin, 2005; Model et al., 1997).

The same regulatory principles orchestrate the Gram-positive CESR, as outlined schematically in Fig. 1.2. Since envelope stress occurs outside the cytoplasm, all systems are comprised of transmembrane sensory components that detect their stimulus in the extracellular space. As for the Gram-negative bacteria, TCS and ECF $\sigma$ factors are at the core of the Gram-positive envelope stress response. Both systems are functionally analogous in that they consist of two proteins, a membrane-anchored sensory component (sensor histidine kinase or anti- $\sigma$ factor, respectively), and a cytoplasmic transcriptional regulator (response regulator or ECF $\sigma$ factor, respectively). In both cases, the regulator is usually kept inactive in the absence of inducing conditions. Upon perceiving envelope stress by the sensory 
component, the regulators become activated and direct (normally up-regulate) the expression of their target genes. The two regulatory principles differ in the mechanism by which the sensor and regulator proteins communicate with one another.

In the case of TCS (Fig. 1.2, middle), activation of the response regulator (RR) by its cognate sensor histidine kinase $(\mathrm{HK})$ is based on the transfer of a phosphoryl group from a donor phospho-histidine in the C-terminal transmitter domain of the HK to an acceptor aspartate in the N-terminal receiver domain of the RR (Parkinson, 1993).

In contrast, communication between the anti- $\sigma$ factor and its corresponding ECF $\sigma$ factor is based on direct protein-protein interactions. In the absence of stress conditions, the anti- $\sigma$ factor tightly binds the ECF $\sigma$ factor, thereby keeping it inactive (Fig. 1.2, left). Under inducing conditions, the ECF $\sigma$ factor is released, either by a conformational change or regulated proteolysis of the anti- $\sigma$ factor. The $\sigma$ factor becomes available for recruitment by RNA polymerase core enzyme to redirect transcription initiation to its specific target promoters, ultimately resulting in the upregulation of its regulon (Helmann, 2002).

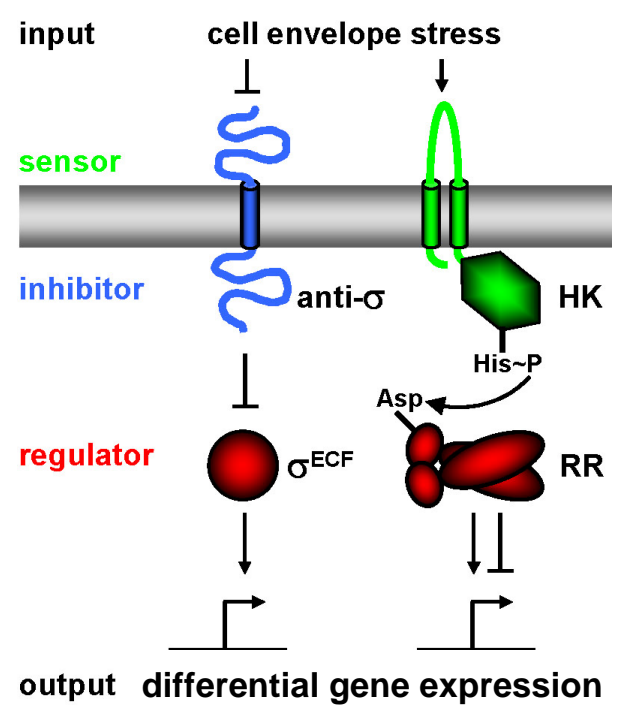

Figure 1.2. Regulatory principles orchestrating cell envelope stress response in Bacillus subtilis. Left: ECF $\sigma$ factors, right: two-component systems $(\mathrm{HK}=$ histidine kinase; $\mathrm{RR}=$ response regulator). Sensor proteins are shown in green, inhibitor proteins in blue, transcriptional regulators in red. Arrows indicate activation, T-shaped lines repression. See text for details.

A distinct phage-shock protein system is absent in Gram-positive bacteria. Instead, its core component, the PspA protein, seems to be embedded in TCS-mediated envelope stress response, at least in the genera Bacillus and Listeria (Jordan et al., 2006). 


\section{The regulatory network orchestrating cell envelope stress response in}

\section{Bacillus subtilis: a case study}

Over the last several years, a detailed picture of the CESR emerged for B. subtilis. Many of the underlying studies addressed the response of this Gram-positive model bacterium to cell wall antibiotics by applying transcriptomics approaches to identify the corresponding stimulons (= all genes that are differentially expressed, usually up-regulated, in the presence of a specific stimulus). Subsequent work identified the regulatory systems that orchestrate this response, thereby dissecting the stimulons into discrete regulons (Fig. 1.3). Initial work from the Helmann laboratory aimed to identify inducers of the ECF $\sigma$ factor $\sigma^{\mathrm{W}}$ amongst cell envelope perturbating agents (Cao et al., 2002b). Vancomycin was identified as the strongest stimulus and used for subsequent in-depth transcriptional profiling. In addition to $\sigma^{\mathrm{W}}$, three other ECF $\sigma$ factors were found to be induced by vancomycin, namely $\sigma^{\mathrm{M}}, \sigma^{\mathrm{V}}$, and $\sigma^{\mathrm{Y}}$, the last two being induced only weakly (Cao et al., 2002b).

Another study from the same group analyzed the bacitracin stress response, which is orchestrated by an even larger number of signal transducing systems (Mascher et al., 2003). In addition to $\sigma^{\mathrm{M}}$ and the $\sigma^{\mathrm{B}}$-mediated general stress response (Price, 2002), three TCS respond to the extracellular presence of bacitracin. The LiaRS TCS is strongly induced by both vancomycin and bacitracin. The paralogous TCSs BceRS and YvcPQ both specifically regulate the expression of an $\mathrm{ABC}$ transporter, which - in case of the Bce system - confers high level bacitracin resistance (Mascher et al., 2003; Ohki et al., 2003a). A second bacitracin resistance determinant, the undecaprenyl pyrophosphate phosphatase BcrC (Bernard et al., 2005 ) under the dual control of two ECF $\sigma$ factors, $\sigma^{\mathrm{M}}$, and $\sigma^{\mathrm{X}}$ (Cao and Helmann, 2002; Ohki et al., 2003b), was also induced by bacitracin.

More recently, the CESR of B. subtilis was exploited further with regard to $\beta$-lactams, Dcycloserine, fosfomycin, and CAMPs (Hutter et al., 2004; Pietiäinen et al., 2005). The transcriptional profiles of the first three compounds were part of a broader panel aimed to apply stimulon patterns for predicting the mechanism of action of unknown compounds, and not analyzed in detail (Hutter et al., 2004). In contrast, the work on CAMPs provided further insights and helped to complete the picture of the regulatory network orchestrating CESR in B. subtilis. Challenges with two naturally occurring cationic peptides, human LL-37 and porcine PG-1, and a synthetic analog, poly-L-lysine, provoked distinct response patterns, orchestrated by three ECF $\sigma$ factors $\left(\sigma^{\mathrm{M}}, \sigma^{\mathrm{W}}\right.$, and $\left.\sigma^{\mathrm{X}}\right)$, the LiaRS TCS and another BceRS homolog, the YxdJK TCS (Pietiäinen et al., 2005). The latter specifically responded to LL-37 only, without conferring resistance against this compound. This study also indicated a 
significant and surprising amount of cross-dependency between TCS- and ECF-mediated responses, even though no direct regulatory overlap has ever been observed. Deletion of ECF $\sigma$ factors strongly reduced the TCS-mediated response to CAMPs. This observation is reminiscent of the results obtained earlier for the alkaline shock response, where a similar link between $\sigma^{\mathrm{W}}$ and the LiaRS-dependent gene expression was already observed (Wiegert et al., 2001). The reason for this interference in signal transduction is unclear at the moment.

ECF $\sigma$ factors involved in orchestrating CESR of B. subtilis. The ECF (or Group 4) $\sigma$ factors belong to the $\sigma^{70}$ family of bacterial $\sigma$ factors (Helmann, 2002; Lonetto et al., 1994). They share a number of common features: (i) They are small proteins, harboring only two of the four conserved regions of the primary $\sigma$ factors, namely region 2 and region 4; (ii) They recognize promoters with a highly conserved 'AAC' motif in the -35 region; (iii) They are usually cotranscribed with their cognate anti- $\sigma$ factor, a transmembrane protein; (iv) They are often involved in regulating functions associated with some aspect of the cell envelope or transport processes (Butcher et al., 2007; Helmann, 2002). The genome of B. subtilis encodes seven ECF $\sigma$ factors (Helmann and Moran, 2002), of which three $\left(\sigma^{\mathrm{M}}, \sigma^{\mathrm{W}}\right.$, and $\left.\sigma^{\mathrm{X}}\right)$ play a role in orchestrating CESR.

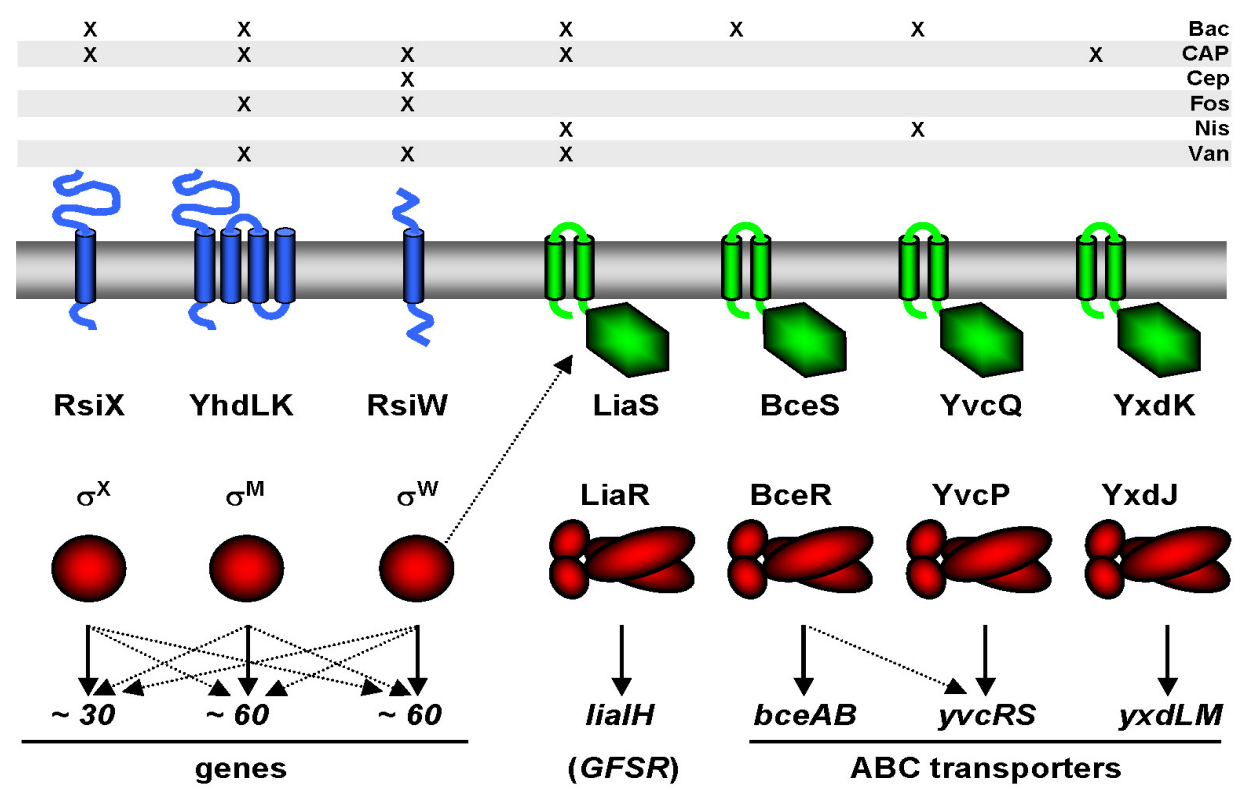

Figure 1.3. The regulatory network of cell envelope stress response in Bacillus subtilis. The same symbols and color-code was applied as in Fig. 1. Dotted lines indicate cross-regulation. The antibiotic specificity for each system is shown above. $\mathrm{Bac}=$ bacitracin; $\mathrm{CAP}=$ cationic antimicrobial peptides (note that individual peptides will only induce a certain subset of the regulators indicated); Cep = cephalosporin $\mathrm{C}$; Fos = fosfomycin; Nis = nisin; Van = vancomycin. It should be noted that because of the known regulatory overlap between ECF $\sigma$ factors, an clear assignment of the inducer spectrum, is not as unambiguously possible as indicated in this figure. See text for details. 
$\sigma^{\mathbf{W}}$ is the best-understood ECF $\sigma$ factor in B. subtilis (Helmann, 2006). It is induced by a number of cell wall antibiotics, such as vancomycin, cephalosporin C, and the CAMPs LL-37 and PG-1, but also by alkaline shock (Cao et al., 2002b; Pietiäinen et al., 2005; Wiegert et al., 2001). Promoter consensus search, in combination with in vivo and in vitro approaches identified $\sim 30$ target promoters (controlling about 60 genes) that are expressed (at least partially) in a $\sigma^{\mathrm{W}}$-dependent manner (Cao et al., 2002a; Huang et al., 1999). It was postulated that $\sigma^{\mathrm{W}}$ controls an 'antibiosis' regulon, based on the inducer spectrum and the putative function of many of its target genes (Helmann, 2002). This hypothesis was subsequently confirmed by studies demonstrating that $\sigma^{\mathrm{W}}$-controlled genes confirm resistance against fosfomycin (Cao et al., 2001), as well as a number of antimicrobial compounds synthesized by closely related Bacillus species (Butcher and Helmann, 2006). Moreover, $\sigma^{\mathrm{W}}$ also provides resistance to the antimicrobial protein SdpC (Butcher and Helmann, 2006), which is produced by sporulating B. subtilis cells to lyse non-sporulating sibling cells in a process termed cannibalism (Ellermeier et al., 2006; Gonzalez-Pastor et al., 2003).

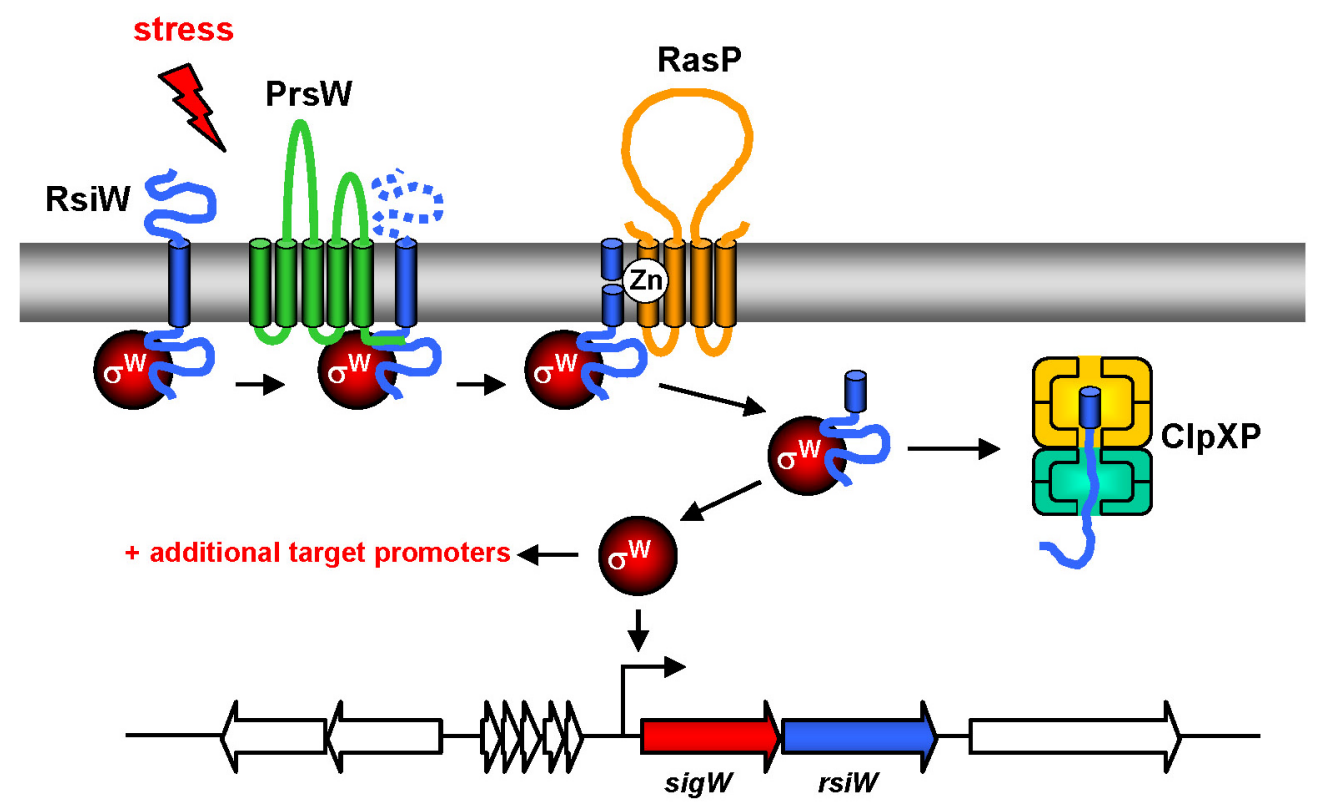

Figure1. 4. Signal transduction mediated by ECF $\sigma$ factors. The proteolytic cascade leading to anti- $\sigma$ factor degredation and ECF $\sigma$ factor activation is illustrated. For color-code, see legend to figure 1. The stimulus is represented by the red arrow. Additional proteins are shown in yellow. See text for further details. This figure is based on a presentation kindly provided by Thomas Wiegert.

The sig $W$ gene is co-transcribed with $r s i W$, encoding the cognate membrane-anchored anti- $\sigma$ factor. A direct protein-protein interaction between $\sigma^{\mathrm{W}}$ and RsiW was verified by yeast twohybrid analysis (Yoshimura et al., 2004). Like E. coli $\sigma^{\mathrm{E}}, \sigma^{\mathrm{W}}$ activation is based on the 
regulated proteolytic degradation of its cognate anti- $\sigma$ factor RsiW. Three consecutive proteolytic steps cleave first the extracytoplasmic, then the membrane-spanning, and finally the cytoplasmic domains of RsiW, respectively (Fig. 1.4). This proteolytic cascade is initiated by PrsW-dependent site-1 cleavage (Ellermeier and Losick, 2006; Heinrich and Wiegert, 2006). PrsW, a membrane-anchored novel protease with 5 transmembrane helices is therefore the prime candidate for the real sensory module in $\sigma^{\mathrm{W}}$-dependent signaling. Subsequently, site-2 cleavage by RasP-mediated regulated intramembrane proteolysis (homologous to $E$. coli RseP) generates a soluble N-terminal fragment of RsiW (Schöbel et al., 2004). The latter is degraded by the cytoplasmic ClpXP proteolytic complex (Zellmeier et al., 2006), thereby ultimately releasing the active $\sigma$ factor, again similar to the mode of $\sigma^{\mathrm{E}}$-activation in $E$. coli (Ades, 2004).

$\sigma^{\mathbf{x}}$ was the first ECF $\sigma$ factor to be studied in detail in B. subtilis. Its gene is co-transcribed with $r$ siX, encoding the corresponding anti- $\sigma$ factor, and primarily expressed in logarithmic and early stationary phase (Huang et al., 1997). Interaction between the $\sigma$ factor:anti- $\sigma$ pair could be demonstrated by yeast two-hybrid system (Yoshimura et al., 2004). Its regulon consists of $~ 30$ genes, organized in 15 transcriptional units (Cao and Helmann, 2004). So far, the major physiological role of $\sigma^{\mathrm{X}}$ is the modulation of the overall envelope net charge, due to the regulation of the dltABCDE and the pssA-ybfM-psd operons. The products of the $d l t$ operon introduce positively charged amino groups into TAs, thereby lowering the overall negative net charge of the cell wall (Perego et al., 1995). A comparable role is exhibited by PssA/Psd, which together catalyze the synthesis of the neutral lipid phosphatidylethanolamine. Since the cytoplasmic membrane has a negative net charge due to the abundance of anionic phospholipids, increased incorporation of neutral lipids therefore also lowers the overall negative net charge (Cao and Helmann, 2004). Altering the overall net charge of the cell envelope has been shown to affect both autolysis and resistance to CAMPs. Consequently, a sig $X$ mutant has an increased rate of autolysis and is more sensitive to CAMPs (Cao and Helmann, 2004).

$\sigma^{\mathbf{M}}$ is also co-transcribed with its negative regulators, encoded by $y h d L$ and $y h d K$ that together form the anti- $\sigma$ complex (Horsburgh and Moir, 1999): a direct protein-protein interaction between $\sigma^{\mathrm{M}}$ and the $\mathrm{N}$-terminal fragment of YhdL could be demonstrated, as well as specific interactions between the two membrane proteins YhdL and YhdK (Yoshimura et al., 2004). The sigM-yhdLK operon is maximally expressed in early to mid-logarithmic growth phase (Horsburgh and Moir, 1999). It is induced by cell wall antibiotics, such as bacitracin, vancomycin or fosfomycin, but also by acidic $\mathrm{pH}$, heat, ethanol, and superoxide stress 
(Thackray and Moir, 2003), and confers resistance to bacitracin, and high salinity (Cao and Helmann, 2002; Horsburgh and Moir, 1999; Mascher et al., 2003). In the B. subtilis strain $\mathrm{W} 23, \sigma^{\mathrm{M}}$ is induced by phosphate depletion and involved in TA biosynthesis (Minnig et al., 2005). A study of $12 \sigma^{\mathrm{M}}$ target promoters in the reference strain $\mathrm{W} 168$ has been published recently (Jervis et al., 2007), but preliminary in vitro data identified many more target genes (John Helmann, personal communication).

A significant amount of regulatory overlap between the target genes of all three ECF $\sigma$ factors has been demonstrated both in vivo and in vitro, with many promoters being recognized by two, or even all three ECF $\sigma$ factors (Cao and Helmann, 2002; Huang et al., 1998; Qiu and Helmann, 2001). It can be envisioned that target gene discrimination (from the available pool of genes preceded by an ECF-type promoter) is therefore a combined result of the timing of expression of individual ECF $\sigma$ factors during the life cycle and the presence of specific inducing conditions, rather than promoter selectivity based on sequence preference alone. This overlap is not only restricted to the expression of individual target genes, but also manifests itself in the regulation of complex phenotypes, such as overall cell envelope integrity, pellicle formation and colony morphology (Mascher et al., 2007). Moreover, in $B$. subtilis W23 the concerted action of $\sigma^{\mathrm{X}}$ and $\sigma^{\mathrm{M}}$ is required for septum formation and cell wall biosynthesis (Minnig et al., 2003). Both ECF $\sigma$ factors together regulate the synthesis of wall TAs, which consist of poly(ribitol phosphate) in this strain, in contrast to the poly(glycerol phosphate)-containing TAs of the sequenced reference strain B. subtilis W168 (Lazarevic et al., 2002).

Cell envelope stress-sensing TCS of $\boldsymbol{B}$. subtilis. The transcriptomics approaches described above identified four TCS that respond to some aspect of cell envelope stress in B. subtilis, with three being induced by bacitracin alone. During the in-depth analysis of the regulatory network orchestrating the bacitracin response, it was noticed that all TCS involved share some overall similarities with regard to the domain architecture of their HKs. These membraneanchored sensor kinases are characterized by a very short $\mathrm{N}$-terminal input domain, consisting solely of two deduced transmembrane helices with hardly any periplasmic linker (less than 10 amino acids for most) in between (Mascher et al., 2003). Subsequent comparative genomics analysis revealed that these so-called intramembrane-sensing HKs are widespread and conserved in Firmicutes bacteria, but can also be found in the Actinobacteria (Hutchings et al., 2004; Mascher, 2006b). Two conserved sub-groups can be distinguished in Firmicutes bacteria, and both groups are involved in mediating CESR in B. subtilis (Mascher, 2006b): (1) 
LiaRS-like three-component systems, and (2) BceRS-like TCS that are functionally linked to $\mathrm{ABC}$ transporters (Fig. 1.5).

The LiaRS TCS - a cell envelope stress-sensing three-component system. This TCS was originally identified as part of the complex regulatory network orchestrating the bacitracin stress response in B. subtilis (Mascher et al., 2003). It also strongly responds to the external presence of other cell wall antibiotics that interfere with the lipid II cycle, such as ramoplanin, vancomycin, or CAMPs (Mascher et al., 2004; Pietiäinen et al., 2005). LiaRS-dependent gene expression is also induced by alkaline shock, detergents, ethanol, phenol, organic solvents, and secretion stress, albeit to a lesser extent (Hyyryläinen et al., 2005; Mascher et al., 2004; Petersohn et al., 2001; Pietiäinen et al., 2005; Tam le et al., 2006; Wiegert et al., 2001). Moreover, its activity is influenced by the density of the negative net charge of the cell envelope: The LiaRS system responds more strongly to the CAMP LL-37 and secretion stress in cells defective in the Dlt system, which has an overall higher negative net charge due to its inability to incorporate D-alanine into its TAs (Hyyryläinen et al., 2007).
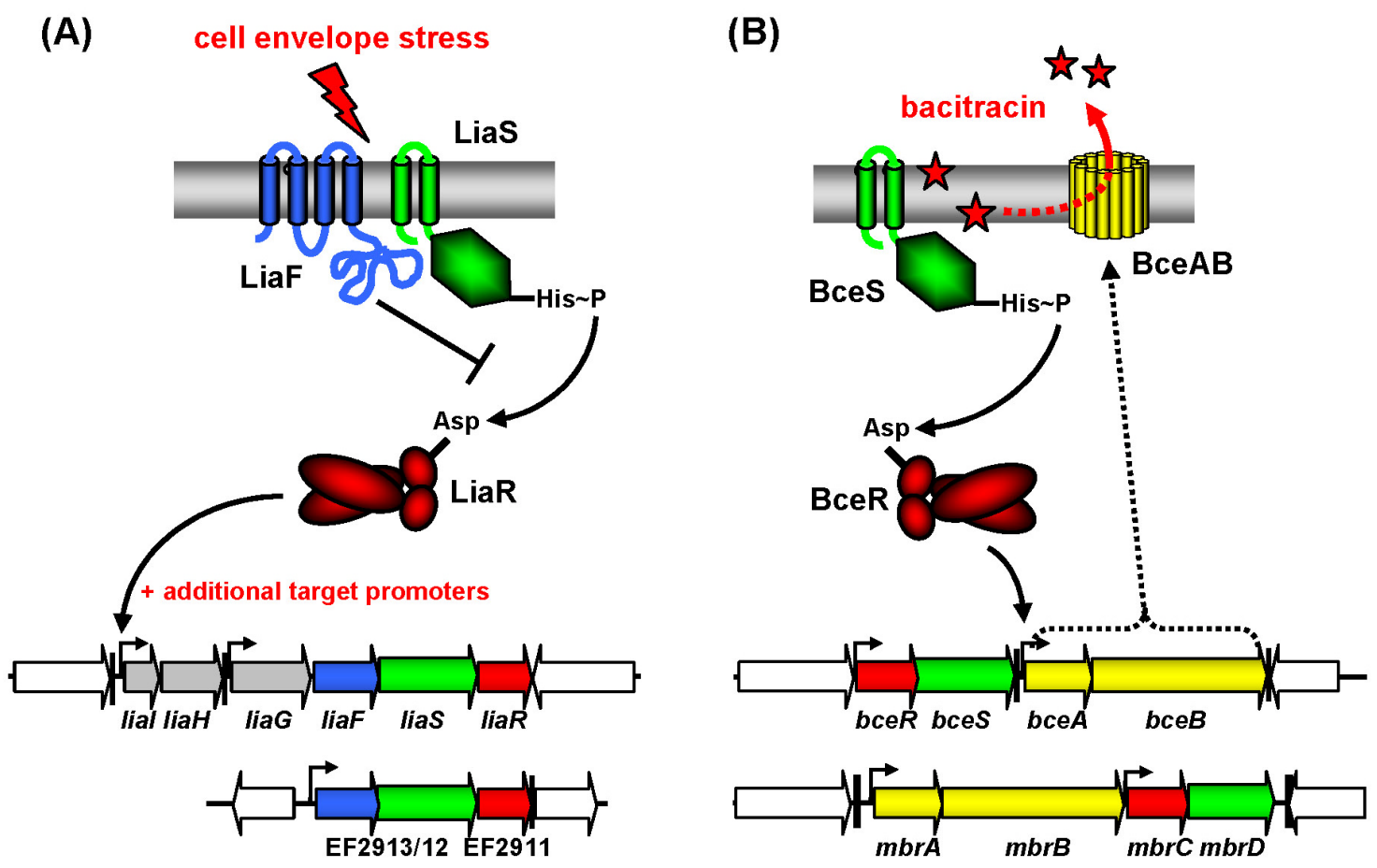

Figure 1.5. Cell envelope stress-sensing two-component systems in Firmicutes bacteria. (A) LiaFSR-like three-component systems. (B) BceRS-BceAB-like TCS-ABC transporter connection. Symbols and color-code as before. See text for details. This figure was taken from (Mascher, 2006b), with modifications. 
The LiaRS TCS is functionally and genetically linked to a third protein, LiaF, which acts as a strong inhibitor of LiaR-dependent gene expression (Jordan et al., 2006) (Fig. 1.5A). The LiaRS-LiaF three-component system is conserved by sequence, genomic context and function in Gram-positive bacteria with a low G+C content (Jordan et al., 2006; Mascher, 2006b). The lia locus consists of six genes, liaIH-liaGFSR. A basal expression level of the last four genes, liaGFSR, encoding the three-component system (liaFSR) and a putative membrane-anchored hypothetical protein $(l i a G)$, is ensured by a weak constitutive promoter upstream of liaG. In contrast, expression of the liaIH operon from $\mathrm{P}_{\text {lial }}$ is completely LiaR-dependent (Jordan et al., 2006).

In B. subtilis, only two promoters are known to be regulated by the LiaRS TCS: the liaI promoter $\left(\mathrm{P}_{\text {lial }}\right)$ and the $y h c Y$ promoter (Jordan et al., 2006), with $\mathrm{P}_{\text {lial }}$ being the primary target. $\mathrm{P}_{\text {lial }}$ is tightly regulated: in the absence of a stimulus, it is virtually switched off, while addition of bacitracin results in an about 200-fold induction (Mascher et al., 2003; Mascher et al., 2004). In contrast to $\mathrm{P}_{\text {lial }}$, a LiaR-dependent $\mathrm{P}_{y h c Y}$-activity was only observed in a liaF mutant, i.e. in the absence of the LiaRS-inhibitor protein (Jordan et al., 2006; Mascher et al., 2004).

$\mathrm{P}_{\text {lial }}$ is also induced in the absence of exogenous stimuli at the onset of stationary phase (Jordan et al., 2007). This time point in the B. subtilis life cycle is characterized by the initiation of a complex regulatory cascade that allows Bacillus to adapt to worsening living conditions, which can ultimately lead to the formation of dormant endospores (Errington, 2003; Msadek, 1999; Phillips and Strauch, 2002). It was demonstrated that $\mathrm{P}_{\text {lial }}$ is directly repressed by binding of the transition state regulator AbrB within the promoter sequence, thereby acting as a roadblock to prevent premature $\mathrm{P}_{\text {lial }}$ activity during logarithmic growth. AbrB repression is released during the transition state by Spo0A, the master regulator of sporulation and $\mathrm{P}_{\text {lial }}$ is induced by an unidentified endogenous stimulus, resulting in the expression of the liaIH operon. While AbrB-binding is sufficient to inhibit the endogenous growth-dependent induction of $\mathrm{P}_{\text {lial }}$, it can be bypassed completely by exogenous induction with cell wall antibiotics. Taken together, LiaRS-dependent gene expression is embedded in transition phase regulation in B. subtilis, and the activity of its primary target promoter, $\mathrm{P}_{l i a l}$, is controlled by at least five regulators.

In contrast to the detailed knowledge on the mechanism of LiaFSR-dependent signal transduction, the physiological role of its primary target genes, liaIH, is largely unknown. LiaI is a small hydrophobic protein of unknown function with two putative transmembrane helices. LiaH is a member of the phage-shock protein family. While the strong induction of 
liaIH (and to a lesser degree also liaGFSR) by cell envelope stress is well documented (see above), mutational analyses of the lia locus so far failed to identify strong phenotypes associated with them, and deletion of lia genes did not alter the sensitivity of the corresponding mutants to the known inducers of the Lia system.

BceRS and its paralogs - a TCS-ABC transporter connection. A regulatory connection between TCS and ABC transporters encoded by genes located directly downstream, was already described some years ago for the Bacillus/Clostridium group, and the authors demonstrated a TCS-dependent expression of the ABC transporter genes for B. subtilis (Joseph et al., 2002). Its genome harbors three such TCS-ABC transporter modules, bceRS$b c e A B$ (formerly $y t s A B C D$ ), $y v c P Q-y v c R S$, and $y x d J K-y x d L M$ (Joseph et al., 2002; Mascher, 2006b).

The BceRS-BceAB system is the best-understood of these detoxification modules (Fig. 1.5B). The BceRS TCS specifically responds to the extracellular presence of bacitracin. Its activation leads to binding of phosphorylated BceR to an inverted repeat upstream of the bceA promoter, resulting in a strong up-regulation of $b c e A B$ expression. The encoded $A B C$ transporter is an efficient bacitracin resistance determinant and is thought to facilitate its removal (Mascher et al., 2003; Ohki et al., 2003a). Recently, it was demonstrated that the HK $\mathrm{BceS}$ alone is unable to sense bacitracin. Instead, the corresponding $\mathrm{ABC}$ transporter $\mathrm{Bce} \mathrm{AB}$ is crucial for bacitracin perception. Moreover, ATP binding/hydrolysis by the nucleotidebinding subunit BceA is a prerequisite for stimulus perception, indicating that BceRS responds to bacitracin transport by $\mathrm{Bce} A \mathrm{~B}$, rather than the extracellular presence of this antibiotic (Rietkötter and Mascher, 2007).

Only very little is known about the other two systems. The YxdJK-YxdLM system has been analyzed genetically, without gaining insights into its biological role (Joseph et al., 2004). Again, the expression of $y x d L M$, encoding the $\mathrm{ABC}$ transporter, is strictly dependent on the corresponding RR YxdJ. More recently, the human CAMP LL-37 was identified as a strong inducer of $y x d L M$ expression (Pietiäinen et al., 2005). Therefore, it is tempting to speculate that YxdJK-YxdLM functions as a CAMP-specific detoxification module. But the exact nature of its substrate remains to be identified.

Induction of $y v c R S$ expression was shown to be induced by bacitracin and nisin (Hansen et al., 2007; Mascher et al., 2003). While the bacitracin induction is weak and presumably indirect (Rietkötter and Mascher, 2007), the nisin-dependent upregulation could point towards 
a role of this module in mediating resistance against some lantibiotic. But again, this assumption needs to be verified.

\section{Signal-transducing systems orchestrating cell envelope stress response in other Gram-positive bacteria}

In the following paragraphs, we will summarize the knowledge of cell envelope stress-sensing regulatory systems in other Gram-positive bacteria. The first sections will address systems homologous to those of B. subtilis, i.e. ECF $\sigma$ factors, and TCS with intramembrane-sensing HKs (LiaRS- and BceRS-like TCS) (Table 1.1).

\section{Cell envelope stress-sensing ECF $\sigma$ factors in other Gram-positive bacteria}

In contrast to the situation for TCS, disappointingly little is known on ECF $\sigma$ factors beyond B. subtilis. A quick glance on the distribution and presence of ECF $\sigma$ factors in Gram-positive genomes shows that these regulators are abundant in most Actinobacteria, whereas their presence is very heterogeneous in the Firmicutes bacteria (Staron et al., 2007)(Table 1.1). All Bacillus species are rich in ECF $\sigma$ factors, while these regulators are almost absent in the lactic acid bacteria and other cocci (Table 1.1). In addition to $S$. coelicolor $\sigma^{\mathrm{E}}$, only one study addressed the role of ECF $\sigma$ factors in Gram-positive CESR, so far. The genome of $B$. licheniformis, a close relative of $B$. subtilis, contains nine ECF $\sigma$ factors, of which six are direct orthologs to the B. subtilis proteins $\sigma^{\mathrm{M}}, \sigma^{\mathrm{V}}, \sigma^{\mathrm{W}}, \sigma^{\mathrm{X}}, \sigma^{\mathrm{Y}}$, and $\sigma^{\mathrm{ylaC}}$ (Wecke et al., 2006). A homolog of the seventh ECF $\sigma$ factor from B. subtilis, $\sigma^{\mathrm{Z}}$, is absent in the genome of $B$. licheniformis. The three novel ECF $\sigma$ factors where designated $\sigma^{\text {ecfG }}, \sigma^{\text {ecfH }}$, and $\sigma^{\text {ecfI }}$. In-depth transcriptional profiling demonstrated that seven of the nine ECF $\sigma$ factors respond to cell envelope stress in this organism: Six were significantly induced by vancomycin $\left(\sigma^{\mathrm{M}}, \sigma^{\mathrm{V}}, \sigma^{\mathrm{W}}\right.$, $\sigma^{\mathrm{X}}, \sigma^{\mathrm{Y}}$, and $\left.\sigma^{\mathrm{ecfH}}\right)$, three by bacitracin $\left(\sigma^{\mathrm{M}}, \sigma^{\mathrm{V}}, \sigma^{\mathrm{Y}}\right)$, while $\sigma^{\text {ecfG }}$ specifically responded to $\beta$ lactams (Wecke et al., 2006). The contribution of these regulators to mediating resistance against these antibiotics has not yet been explored.

\section{LiaFSR-like three-component systems: conserved cell envelope stress- sensors in Firmicutes bacteria}

LiaFSR-homologous three-component systems are widespread amongst the Firmicutes bacteria, with the noteworthy exception of the genera Lactobacillus and Clostridium (Table 1.1). Two groups can be distinguished, based on the genomic context of the corresponding 
Table 1.1. Distribution and conservation of cell envelope stress-sensing regulatory systems in the genomes of Firmicutes bacteria

\begin{tabular}{|c|c|c|c|c|c|}
\hline \multirow[b]{2}{*}{ Organism } & \multirow[b]{2}{*}{ Size $[\mathrm{Mb}]^{1}$} & \multirow[b]{2}{*}{$\mathbf{E C F}^{2}$} & \multirow[b]{2}{*}{$\Sigma^{1}$} & \multicolumn{2}{|c|}{$\begin{array}{l}\text { Two-component systems with } \\
\text { intramembrane-sensing HK }\end{array}$} \\
\hline & & & & LiaRS $^{4}$ & BceRS $^{4}$ \\
\hline Bacillus anthracis & $5.23-5.5$ & 16 & $47-50$ & $1(\mathrm{I})$ & 1 \\
\hline B. cereus & $5.43-5.84$ & $13-18$ & $45-50$ & $1(\mathrm{I})$ & 5 \\
\hline B. clausii & 4.3 & 4 & 40 & $1(\mathrm{I})$ & 4 \\
\hline B. halodurans & 4.2 & 9 & 45 & $1(\mathrm{I})$ & 5 \\
\hline B. licheniformis & 4.22 & 9 & $34-35$ & $1(\mathrm{I})$ & 4 \\
\hline B. subtilis & 4.21 & 7 & 33 & $1(\mathrm{I})$ & 3 \\
\hline B. thuringiensis & 5.31 & 16 & $50-51$ & $1(\mathrm{I})$ & 3 \\
\hline Clostridium acetobutylicum & 4.13 & 3 & 40 & - & 4 \\
\hline C. difficile & 4.3 & 2 & 54 & - & 7 \\
\hline C. perfringens & $2.96-3.26$ & 4 & $18-24$ & - & 2 \\
\hline C. tetani & 2.87 & 10 & 28 & - & 2 \\
\hline Desulfitobacterium hafiense & 5.73 & $20-23$ & 70 & - & 5 \\
\hline Enterococcus faecalis & 3.36 & 2 & 18 & 1 (II) & 1 \\
\hline Geobacillus kaustophilus & 3.59 & 3 & 27 & $1(\mathrm{I})$ & 3 \\
\hline Lactobacillus acidophilus & 1.99 & - & 8 & - & 1 \\
\hline L. brevis & 2.34 & - & 10 & - & - \\
\hline L. casei & 2.92 & - & 17 & - & 5 \\
\hline L. delbrueckii bulgaricus & 1.86 & 1 & $6-7$ & - & - \\
\hline L. gasseri & 1.89 & - & 5 & - & - \\
\hline L. johnsonii & 1.99 & - & 9 & - & - \\
\hline L. plantarum & 3.35 & - & 15 & - & - \\
\hline L. sakei $23 K$ & 1.88 & - & 10 & 1 (II) & 1 \\
\hline L. salivarius & 1.83 & - & 6 & - & - \\
\hline Lactococcus lactis & $2.37-2.6$ & 1 & $7-9$ & 1 (II) & 1 \\
\hline Leuconostoc mesenteroides & 2.08 & - & 10 & - & - \\
\hline Listeria innocua & 3.09 & 1 & 17 & $1(\mathrm{I})$ & 1 \\
\hline L. monocytogenes & $2.91-2.94$ & 1 & $15-16$ & $1(\mathrm{I})$ & 1 \\
\hline Moorella thermoacetica & 2.63 & 3 & 16 & - & - \\
\hline Oceanobacillus iheyensis & 3.63 & 9 & 20 & $2(\mathrm{I} / \mathrm{II})$ & 1 \\
\hline Pediococcus pentosaceus & 1.83 & - & 8 & - & 1 \\
\hline Staphylococcus aureus & $2.74-2.9$ & - & $14-17$ & 1 (II) & 2 \\
\hline S. epidermidis & $2.56-2.64$ & - & 16 & 1 (II) & 2 \\
\hline S. haemolyticus & 2.69 & - & 16 & 1 (II) & 3 \\
\hline S. saprophyticus & 2.58 & - & 11 & 1 (II) & - \\
\hline Streptococcus agalactiae & $2.13-2.21$ & 1 & $20-22$ & 1 (II) & 1 \\
\hline S. mutans & 2.03 & - & 14 & 1 (II) & 1 \\
\hline S. pneumoniae & $2.04-2.16$ & - & 14 & 1 (II) & - \\
\hline S. pyogenes & $1.84-1.94$ & - & $12-14$ & 1 (II) & - \\
\hline S. thermophilus & $1.8-1.86$ & - & $8-10$ & 1 (II) & 1 \\
\hline Thermoanaerobacter tengcongensis & 2.69 & 6 & 20 & - & - \\
\hline
\end{tabular}

${ }^{1}$ Information on genome size and total numbers of TCS is derived from the MiST (Ulrich and Zhulin, 2007) and Genome Atlas (Pedersen et al., 2000; Hallin and Ussery, 2004; Kiil et al., 2005b) databases at http://genomics.ornl.gov/mist/ and http://www.cbs.dtu.dk/services/GenomeAtlas/index.php, respectively.

${ }^{2}$ Numbers of ECF $\sigma$ factors are derived from a comprehensive ECF database and classification system, and the $\sigma$ factor census published in Genome Atlas (Kiil et al., 2005a; Staron et al., 2007).

${ }^{3}$ Identification of specific TCS in bacterial genomes is based on genomic BLAST searches (blastp) of either the complete proteins at http://www.ncbi.nlm.nih.gov/sutils/genom table.cgi or - where applicable - signature domains thereof, such as the extracytoplasmic senor domains of HK (i.e for CiaRH), to increase the specificity of the search. Moreover, genomic context conservation was also used as a criterion, where applicable (LiaRS, BceRS, VanRS, YycFG, LytSR), using the MicrobesOnline (Alm et al., 2005) or The SEED databases at http://www.microbesonline.org/ and http://theseed.uchicago.edu/FIG/index.cgi, respectively.

${ }^{4}$ Identification of TCS with intramembrane-sensing histidine kinases is derived from a comparative genomics analysis (Mascher 2006). Two groups of LiaRS-like TCS can be distinguished, based on their genomic context. Group I, liaIH(G)FSR; Group II, liaFSR. 
loci (Jordan et al., 2006; Mascher, 2006b) (Fig. 1.5A). LiaRS-homologs in bacilli (Group I) are embedded in the liaIH-(G)FSR locus (with a liaG-like gene only present in B. subtilis, $B$. licheniformis and B. halodurans). Group II only shows a conservation of the liaFSR locus. In Listeria species, liaIH- and liaFSR-like genes are organized as two separate operons, but both still seem to be under the transcriptional control of the LiaRS systems (Jordan et al., 2006). Therefore, they are also listed as group I (Table 1.1). This difference in genomic context also seems to indicate a different cellular role for these systems. Based on the available data, TCS embedded in lialH-(G)FSR-like loci primarily (maybe even exclusively) regulate their own expression, whereas Group II LiaRS-like TCS seem to orchestrate much larger regulons and seem to represent the primary cell envelope stress-responding systems in the corresponding organisms. This hypothesis is supported by data from Staphylococcus aureus VraSR and Lactococcus lactis CesSR.

VraSR is the best-understood LiaRS homolog, so far. It was originally identified as an upregulated locus in a VRSA (vancomycin-resistant $S$. aureus) strain, compared to a VSSA (sensitive) strain (Kuroda et al., 2000). VraSR was also upregulated in a VISA (vancomycin intermediate resistant $S$. aureus) strain relative to an isogenic VSSA strain (McAleese et al., 2006). The vraSR genes are co-transcribed with two upstream genes, termed orfl and the liaF-homolog $y v q F$, and a VraR-dependent auto-induction of this four gene operon was demonstrated (Yin et al., 2006). The VraSR system is strongly induced by a number of cell wall antibiotics, including vancomycin, teicoplanin, $\beta$-lactams, bacitracin, and D-cycloserine, but not by general stresses, such as heat, osmotic shock or pH shifts (Kuroda et al., 2003). It also responds to sub-lethal perturbations of cell wall biosynthesis caused by the depletion of $p b p B$ (encoding an essential penicillin-binding protein crucial for peptidoglycan crosslinkage) and murF (its gene product catalyzing the last cytoplasmic step in peptidoglycan precursor biosynthesis, addition of the D-alanyl-D-alanine dipeptide to the UDP-linked MurNActripeptide) (Gardete et al., 2006; Sobral et al., 2007). Surprisingly, preliminary data indicate that $S$. aureus VraSR - in contrast to the LiaRS system of B. subtilis - does not respond to CAMPs such as LL-37 (Pietiäinen et al., 2007).

Knock-out of $v r a S R$ resulted in increased susceptibility towards all of its inducers (with the exception of D-cycloserine) and fosfomycin (Gardete et al., 2006; Kuroda et al., 2003). Transcriptional profiling identified 46 genes that were induced by vancomycin in a VraSRdependent manner, including its own locus (positive autoregulation) and a number of genes encoding functions involved in cell envelope biogenesis, such as murZ (peptidoglycan 
monomer precursor biosynthesis), $p b p 2, s g t B$ (peptidoglycan polymerization), or tagA (TA biosynthesis) (Kuroda et al., 2003).

CesSR was originally described as LlkinD/LlrD in a systematic analysis of six TCS from $L$. lactis strain MG1363. These authors were unable to generate an insertion mutant of llkinD, encoding the HK, but the corresponding RR mutant MGRrD showed an increased salt/osmosensitivity (O'Connell-Motherway et al., 2000). Recently, it was shown to respond to the extracellular presence of the lactococcal bacteriocin Lcn972 and renamed CesSR (Martinez et al., 2007) - an unfortunate choice, since it should not be confused with another cell envelope stress-sensing TCS, CesRK of Listeria monocytogenes (Kallipolitis et al., 2003), to which it bears no similarity (see further below for details). Transcriptome analysis revealed that the expression of 26 genes was significantly upregulated in the presence of Lcn972, of which 23 responded in a CesSR-dependent manner. Many of these genes encode putative membrane or stress-related proteins. As with all LiaRS-homologs, the corresponding locus of L. lactis is subject to positive autoregulation, and includes a third liaF-homologous gene, yjbB (llmg1650), which is located directly upstream of cesSR. The CesSR TCS is also induced by other cell wall antibiotics, such as bacitracin, vancomycin, and plantaricin C. CesR disruption results in a slight increase in susceptibility to bacitracin, nisin, and plantaricin C, all of which interfere with the Lipid II cycle (Martinez et al., 2007).

One of the most strongly induced genes of the CesSR regulon is $\operatorname{spxB}$ (formerly yneH), one of seven paralogs of L. lactis that are homologous to B. subtilis Spx (Nakano et al., 2003). SpxB expression and subsequent interaction with RNA polymerase leads to upregulation of oatA, ultimately resulting in increased $O$-acetylation of peptidoglycan, rendering the cells more resistant to autolysis and lysozyme treatment (Veiga et al., 2007). The authors of this study hypothesize that this novel resistance mechanism is induced upon CesS-dependent sensing of cell envelope stress, such as peptidoglycan hydrolysis caused by the presence of lysozyme.

LiaFSR-homologs in other Firmicutes bacteria. Vancomycin treatment of both a sensitive and a tolerant strain of Streptococcus pneumoniae resulted in an upregulation of the SP03850387 locus, encoding the liaFSR-homologous TCS03 (Haas et al., 2005).

Enhanced nisin resistance in Listeria monocytogenes is associated with increased expression of hpk1021 and pbp2229, encoding a LiaS-homologous HK and a penicillin-binding protein, respectively (Gravesen et al., 2001). It could be demonstrated that disruption of both genes abolished the nisin resistance phenotype, and that pbp2229 expression depends on HPK1021 
(Gravesen et al., 2004). Moreover, a mutant harboring an in-frame deletion of the corresponding RR, RR1022, showed a slightly increased ability to invade Cos-1 fibroblast cells compared to the isogenic wild type strain (Williams et al., 2005).

In-depth transcriptional profiling of the CESR in B. licheniformis revealed that the LiaFSRhomologous YvqFEC system is strongly induced by bacitracin and nisin. Weaker induction was observed for vancomycin and D-cycloserine (Wecke et al., 2006).

Systematic inactivation and subsequent phenotypic characterization of TCS in Enterococcus faecalis revealed that a mutant of the LiaR-homologous protein RR03 shows increased bacitracin sensitivity (Hancock and Perego, 2004), indicative for a role of the corresponding TCS in counteracting cell envelope stress.

Taken together, LiaFSR and its homologs are conserved cell envelope stress-sensing threecomponent systems in Firmicutes bacteria that play a crucial role in responding and counteracting damages caused by the extracellular presence of cell wall antibiotics and other perturbations of cell envelope integrity. While this signaling module is clearly conserved, its output is not: It shows a remarkable variability with regard to regulon size and cellular role, indicating that its regulatory features have been 'used' by evolution and adapted to the physiological requirements and life style of the individual organism.

\section{BceRS-like detoxification modules: a TCS-ABC transporter connection conserved in Firmicutes bacteria}

As mentioned above, a regulatory relationship between TCS and ABC transporters has been noticed before in the Bacillus/Clostridium group (Joseph et al., 2002). A recent analysis of Gram-positive genomes demonstrates the predominance of such TCS-ABC transporter modules in these two genera (Table 1.1). Moreover, this group completely overlaps with a conserved sub-group of TCS harboring intramembrane-sensing HKs (Mascher et al., 2003; Mascher, 2006b). Work from B. subtilis established that in these detoxification modules the TCS respond to the extracellular presence of antimicrobial compounds. Upon activation, they upregulate the expression of $\mathrm{ABC}$ transporters, encoded by neighboring (usually downstream) genes (Joseph et al., 2002; Joseph et al., 2004; Ohki et al., 2003a) (Fig. 1.5B). The ABC transporter then facilitates removal of the harmful drug, thereby also removing the initial stimulus of the system, which finally shuts down again (Mascher, 2006b). In contrast to the Lia system, the TCS of the detoxification modules are not autoregulated.

Relatively little is known about these systems beyond B. subtilis, despite their overall abundance. Two bacitracin resistance modules have been described. The BacRS-BcrABC 
module confers bacitracin self-resistance in the producing strain B. licheniformis ATCC10716 (Neumüller et al., 2001; Podlesek et al., 1995). In Streptococcus mutans, a similar module is encoded by the mbrABCD locus (Tsuda et al., 2002). Here, the genes encoding the TCS, $m b r C D$, are located downstream of the genes for the $\mathrm{ABC}$ transporter MbrAB (Fig. 1.5B). The genome of $B$. licheniformis DSM13 encodes four TCS-ABC transporter modules, two of which - ytsABCD and $y x d J$-Bli04268-70 - are induced by bacitracin. The second system also responds to nisin (Wecke et al., 2006). Recently, the GraRS TCS of S. aureus was described to regulate expression of the VraFG ABC transporter, encoded by genes located directly downstream of graSR. This module is involved in resistance to vancomycin and - more pronounced - polymyxin B (Meehl et al., 2007). The genes of another related system, GtcRS of $B$. brevis, are located next to the grs operon encoding the multienzymes involved in the biosynthesis of the peptide antibiotic gramicidin (Turgay and Marahiel, 1995). While no functional characterization has been carried out, a role of this TCS in gramicidin autoimmunity seems likely.

Two unusual examples of BceRS-like TCS were described in Staphylococcus epidermidis and L. monocytogenes. These TCS, while sharing the overall sequence similarities and genomic context conservation (Mascher, 2006b), differ from most TCS of this group by regulating more than one operon encoding an ABC transporter. ApsXRS is a three-component antimicrobial peptide-sensing system in $S$. epidermidis that is conserved in other staphylococci (Li et al., 2007). All three proteins, the TCS ApsRS as well as the third protein, ApsX (its exact function still unknown), are crucial for CAMP sensing. This three-component system responds to a wide range of structurally unrelated CAMPs, including LL-37 ( $\alpha$ helical), $\beta$-defensin 3 (bridged), histatin (His-rich) and the bacterial lantibiotic nisin. Preliminary results indicate that the HK ApsS might sense these CAMPs by direct binding to the 9 amino acid short extracellular loop, which has a high density of negatively charged amino acids ( $\mathrm{Li}$ et al., 2007). But a role of the neighboring ABC transporter, encoded by $v r a F G$, in stimulus perception has not been addressed so far.

A homologous system, VirRS, was described as a TCS critical for the virulence of $L$. monocytogenes (Mandin et al., 2005). It lacks an ApsX homolog and its genomic context differs from the aps system. But the regulons of both systems are almost identical in size and functions. Both regulate the expression of the $d l t$ operon, the $m p r F$ gene, and an operon encoding an $\mathrm{ABC}$ transporter. The first two loci are involved in lowering the net surface charge of the cell envelope, by incorporating D-alanine into TAs, and lysine into membrane 
lipids, respectively. Consequently, both systems play an important role for CAMP resistance (Li et al., 2007; Thedieck et al., 2006).

Taken together, the available data so far identified two sub-groups of BceRS homologous TCS in Firmicutes bacteria. The first group - exemplified by BceRS, MbrAB, YtsAB, GtcRS - is involved in mediating drug-specific resistance against peptide antibiotics, such as bacitracin, by regulating the expression of a neighboring $\mathrm{ABC}$ transporter that facilitates removal. The second group includes ApsXRS and VirRS, which represent important CAMPspecific detoxification systems. These systems - in addition to inducing the expression of an $\mathrm{ABC}$ transporter - lower the overall negative net charge of the cell envelope in response to the extracellular presence of a variety of structurally unrelated CAMPs. 


\section{B. Aims of this work}

The cell envelope stress response of Bacillus subtilis is mediated by seven signal-transducing systems (Mascher et al., 2003). One of these systems is the LiaRS TCS. It is strongly induced by perturbations of the cell envelope, especially the presence of antibiotics that interfere with the lipid II-cycle. Upon induction, the LiaRS TCS activates the expression of the liaIHGFSR operon (Mascher et al., 2004). The primary aim of this work was to unravel the mechanism of LiaRS-dependent signal transduction and gene expression.

Towards that goal, the effect of single gene deletion within the lia locus on $\mathrm{P}_{\text {lial }}$ induction should be studied by $\beta$-galactosidase assays. For this purpose, different reporter strains carrying ectopically integrated $\mathrm{P}_{\text {lial }}$-lac $Z$ fusions had to be constructed and examined. The binding site of LiaR, the response regulator essential for $\mathrm{P}_{\text {lial }}$ activation, should be identified by comparative genomics and confirmed by mutational studies. Based on its sequence, it should be possible to search for additional LiaR target promoters in B. subtilis that, if present, should be investigated further by reporter gene fusions and global transcriptional profiling.

Moreover, the inducer profile of the LiaRS system should be investigated. In addition to the investigation of cell wall antibiotics and other envelope perturbating conditions, the regulation of $\mathrm{P}_{\text {lial }}$ induction during transition state should also be elucidate. Preliminary data indicate that $\mathrm{P}_{\text {lial }}$ is induced at the onset of stationary phase without external addition of cell wall antibiotics. This time point in the life cycle of $B$. subtilis is characterized by the induction of a complex regulatory cascade, orchestrated by the transition state regulator AbrB and the master regulator of sporulation, Spo0A. Therefore, mutants in these and other transition state regulators will be generated and tested for their effect on $\mathrm{P}_{\text {lial }}$ induction. The mode of interaction of positive candidates will be further investigated.

The ultimate aim of this thesis is the identification of the stimulus sensed by the sensor kinase LiaS and the characterization of its mechanism of stimulus perception. Towards that end, a mutational study of LiaS will be initiated, based on sequential deletions, site-specific mutagenesis, and hybrid gene analyses. Mutations that confer altered phenotypes will be further characterized. 


\section{Chapter 2:}

\section{Regulation of LiaRS-Dependent Gene Expression in Bacillus subtilis: Identification of Inhibitor Proteins, Regulator Binding Sites and Target Genes of a Conserved Cell Envelope Stress-Sensing Two-Component System}

The work described in this chapter was published in:

Jordan, S., Junker, A., Helmann, J. D., and Mascher, T. 2006. Regulation of LiaRSdependent gene expression in Bacillus subtilis: Identification of inhibitor proteins, regulator binding sites and target genes of a conserved cell envelope stress-sensing two-component system. J. Bacteriol. 188: 5153-5166

\section{Author contributions:}

Sina Jordan performed the experiments concerning the liaI promoter and draw the figures. Anja Junker performed the experiments concerning the $y h c Y$ promoter. Initial experiments were performed by Thorsten Mascher in the laboratory of John D. Helmann. Sina Jordan and Thorsten Mascher wrote the paper. 


\section{Abstract}

The regulatory network of the cell envelope stress response in Bacillus subtilis involves both extracytoplasmic function (ECF) $\sigma$-factors and two-component signal transducing systems. One such system, LiaRS, responds to cell wall antibiotics that interfere with the undecaprenol cycle and to perturbation of the cytoplasmic membrane. It is encoded by the last two genes of the liaIHGFSR locus. Here, we analyzed the expression of two LiaR-dependent operons, liaIHGFSR and $y h c Y Z-y h d A$, and characterized a palindromic sequence required for LiaRdependent activation. Since induction of the strong liaI promoter leads to both lialH and liaIHGFRS transcripts, LiaR is positively autoregulated. Systematic deletion analysis of the lial operon revealed that $\mathrm{LiaF}$ is a potent negative regulator of LiaR-dependent gene expression: a non-polar liaF deletion led to constitutive activation of both characterized LiaRdependent promoters. The liaF gene is conserved in both sequence and genomic context in the Firmicutes group of Gram-positive bacteria, located directly upstream of liaSR orthologs. LiaH, a homolog of E. coli phage-shock protein A, also plays a more subtle role in negatively modulating the bacitracin-inducible expression from LiaR-dependent promoters. Our results support a model in which the LiaFRS module integrates both positive- and negative-feedback loops to transduce cell envelope stress signals.

\section{Introduction}

Soil is one of the most complex microbial habitats on earth. Nutrient supply varies greatly and on short notice, as do many physicochemical parameters, such as temperature, oxygenconcentration, and moisture. The presence of toxic chemicals and the high population density adds another layer of complexity (Paul and Clark, 1996). Soil bacteria have adapted to this environment in many ways. A broad range of transport systems together with flexible metabolic capabilities allow them to use a variety of nutrient sources. An extensive set of secondary metabolites is thought to suppress the growth of competitors. This trait is specifically pronounced in the actinomycete group of soil bacteria, the most prodigious producers of antimicrobial compounds: two thirds of all antibiotics in clinical use are synthesized by these bacteria alone (Bentley et al., 2002; Thompson et al., 2002). Production 
of and resistance against antibiotics is therefore an important aspect of life in soil (Nwosu, 2001).

The cell envelope is the first and major line of defence against threats from the environment. It gives the cell its shape and counteracts the high inner osmotic pressure (Delcour et al., 1999). It also provides an important sensory interface and molecular sieve between a bacterial cell and its surroundings, mediating both information flow and controlled transport of solutes. Because of its crucial role, it is an attractive target for numerous antibiotics (Bugg and Walsh, 1992; Kahne et al., 2005; Koch, 2003; Silver, 2003; Walsh, 2003). Therefore, monitoring cell envelope integrity is critical for survival.

By applying genome-wide transcript profiling, the regulatory network of the cell envelope stress response in Bacillus subtilis was recently characterized: addition of cell wall inhibitors such as bacitracin (produced by Bacillus spp.) and vancomycin (a secondary metabolite of actinomycetes) induces several transmembrane signal transducing pathways, orchestrated by at least three alternative (ECF-) $\sigma$-factors and four two-component systems (TCS) (Cao et al., 2002b; Mascher et al., 2003). The use of different antibiotics allowed the differentiation between relatively antibiotic-specific (YvcPQ, BceRS in case of bacitracin, $\sigma^{\mathrm{W}}$ for vancomycin) and more general cell envelope stress responses such as $\sigma^{\mathrm{M}}$ and the LiaRS (formerly YvqEC) TCS (Mascher et al., 2003). Interestingly, the sensors of all cell-envelope stress-sensing TCS (BceS, YvcQ and LiaS) appear to define a new family of intramembranesensing histidine kinases. These proteins share an unusually short sensing domain that is almost completely buried in the cytoplasmic membrane. It has been postulated that these kinases detect their signal with their transmembrane helices directly at the membrane interface (Mascher et al., 2003).

The liaIHGFSR locus is expressed from a strictly LiaR-dependent $\sigma^{\mathrm{A}}$-type promoter upstream of the liaI gene $\left(\mathrm{P}_{\text {lial }}\right)$. The LiaRS TCS senses vancomycin, bacitracin, ramoplanin and nisin and mediates a 100- to 1000-fold induction from $\mathrm{P}_{\text {lial }}$ (Mascher et al., 2004). All four drugs interfere with the lipid II cycle in the cytoplasmic membrane, the rate-limiting step

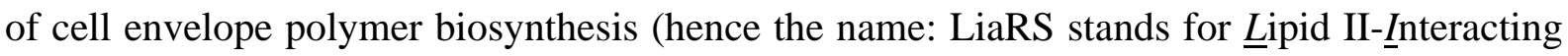
$\underline{A n t i b i o t i c s}$ Response $\underline{R}$ egulator and $\underline{S}$ ensor) (Mascher et al., 2004). A strong stem-loop structure downstream of the second gene, liaH, results in two different transcripts: a major 1.1 $\mathrm{kb}$ mRNA consisting of lialH and a $\sim 4 \mathrm{~kb}$ transcript, including the whole operon. A 74 nucleotide promoter region is fully sufficient for the strong antibiotic-inducible, LiaRdependent activation of gene expression (Mascher et al., 2004). 
Here we identify LiaF, a putative membrane protein, as an integral part of the LiaRS TCS. Deletion of liaF leads to a completely derepressed, stimulus-independent expression from both characterized LiaR-dependent promoter regions. A key role for LiaF as part of a threecomponent signaling system (LiaFRS) is supported by genomic context clustering analysis: the liaFSR gene cluster is conserved in Gram-positive bacteria with a low $\mathrm{G}+\mathrm{C}$ content (Firmicutes). LiaH, a homolog of E. coli phage-shock protein A (PspA), also negatively modulates induction of LiaR-dependent promoters. The minimal bacitracin-inducible promoter fragments controlling expression of both LiaR-dependent operons (liaIHGFSR and $y h c Y Z-y h d A$ ) each harbor a putative LiaR-binding site, identified by comparative genomics and confirmed by mutational studies, that is essential for LiaR-dependent transcription.

\section{Material and Methods}

Bacterial strains and growth conditions. B. subtilis was routinely grown in LB medium at $37^{\circ} \mathrm{C}$ with aeration. All strains used in this study are derivatives of the laboratory wild type strains W168 and CU1065 (W168 trpC2 attSP $\beta$ ) and are listed in Table 2.1. Kanamycin (10 $\mu \mathrm{g} / \mathrm{ml})$, chloramphenicol $(5 \mu \mathrm{g} / \mathrm{ml})$, spectinomycin $(100 \mu \mathrm{g} / \mathrm{ml})$, and tetracyclin(10 $\mu \mathrm{g} / \mathrm{ml})$ were used for the selection of the B. subtilis mutants used in this study. Transformation was carried out as described (Harwood and Cutting, 1990).

Allelic replacement mutagenesis using Long Flanking Homology (LFH-) PCR. This technique is derived from the published procedure (Wach, 1996) and was performed as described previously (Mascher et al., 2003). In brief: resistance cassettes were amplified from a suitable vector as template (Guerout-Fleury et al., 1995; Youngman, 1990). Two primer pairs were designed to amplify $\sim 1000 \mathrm{bp}$ DNA-fragments flanking the region to be deleted at its 5'- and 3'-end. The resulting fragments are here called 'up' and 'do' fragment. The 3'-end of the up-fragment as well as the 5'-end of the do-fragment extended into the gene(s) to be deleted in a way that all expression signals of genes up- and downstream of the targeted genes remained intact. Extensions of $\sim 25$ nucleotides were added to the 5'-end of the 'up-reverse' and the 'do-forward' primers that were complementary (opposite strand and inverted sequence) to the 5'- and 3'-end of the amplified resistance cassette. All obtained fragments were purified using the PCR-purification kit from Qiagen. 100-150 ng of the up- and dofragments and 250-300 ng of the resistance cassette were used together with the specific upforward and do-reverse primers at standard concentrations in a second PCR-reaction. In this 
reaction the three fragments were joined by the 25 nucleotide overlapping complementary ends and simultaneously amplified by normal primer annealing. The PCR-products were directly used to transform $B$. subtilis. Transformants were screened by colony-PCR, using the up-forward primer with a reverse check-primer annealing inside the resistance cassette (Table 2.3). The integrity of the regions flanking the integrated resistance cassettes was verified by sequencing PCR products of $\sim 1000 \mathrm{bp}$ amplified from chromosomal DNA of the resulting mutants. Sequencing was performed in house by the GenoMIK center. All PCR-reactions were done in a total volume of $50 \mu \mathrm{l}(10 \mu \mathrm{l}$ for colony PCR) using the HotStar DNAPolymerase Mastermix (Qiagen) or TripleMaster Polymerase Mix (Eppendorf) according to the manufacturer's procedure. The primers used in this study are listed in Table 2.3.

Construction of transcriptional promoter-lac Z fusions. An ectopic integration of a $\mathrm{P}_{\text {lial }}{ }^{-}$ lacZ fusion was constructed based on the vector pAC6 (Table 2.2). This vector carries a chloramphenicol resistance cassette for selection in B. subtilis, and integrates into the amyE locus by double crossing-over, resulting in a stable integration of $\mathrm{P}_{\text {liar }}$ lac $Z$ fusions (Stülke $e t$ al., 1997). A promoter fragment similar to $\mathrm{P}_{\text {lial-83 }}$ described earlier for pSLZ83 (Mascher et al., 2004) was amplified, using the primers \#99 and \#100, thereby introducing EcoRI and BamHI sites, respectively (Table 2.3). Standard cloning techniques were applied (Sambrook and Russell, 2001). The insert was verified by DNA sequencing at the GenoMIK center, Göttingen. The resulting pAC6-derived plasmid, pTM1 (Table 2.2), was linearized with ScaI and used to transform B. subtilis 168 with chloramphenicol selection, resulting in strain TMB016. Subsequently, individual genes of the lia locus were replaced with a kanamycin resistance cassette by transforming TMB016 with chromosomal DNA from strains HB0920, HB0933 and TMB002-TMB004, resulting in strains TMB017-TMB022 (see Table 2.1 for details). The pAC6 vector was also used to construct $\mathrm{P}_{y h c Y^{-}}$and $\mathrm{P}_{l i a G^{-}}$lacZ fusions, applying the same cloning strategy (Table 2.2). The primers used to are listed in Table 2.3, the resulting strains used in this study are given in Table 2.1.

Complementation of liaF in in-frame deletion mutants. An ectopic integration of a $\mathrm{P}_{x y l^{-}}$ liaF fusion was constructed based on the vector pXT (Derre et al., 2000). This vector is a pDG1782-derivative (Guerout-Fleury et al., 1996) that carries a spectinomycin resistance cassette for selection in B. subtilis, a xylose-inducible promoter for liaF expression, and integrates into the $t h r C$ locus by double crossing-over, resulting in a stable integration of $\mathrm{P}_{x y l^{-}}$ liaF fusions. We amplified liaF with primers \#35 and \#36, thereby introducing EcoRI and HindIII sites, respectively (Table 2.3). Cloning and verification of DNA sequence was performed as described above. The resulting pXT-derived plasmid, pSJ701 (Table 2.2), was 
linearized with ScaI and used to transform B. subtilis TMB027-TMB029 with spectinomycin selection, resulting in strains TMB182-TMB184 (Table 2.1). For liaF expression 0,2\% xylose was added to the medium.

Measurement of induction by $\beta$-galactosidase assay. Cells were inoculated from fresh overnight cultures and grown in LB-medium at $37^{\circ} \mathrm{C}$ with aeration until they reached an $\mathrm{OD}_{600} \approx 0.4$. The culture was split, adding bacitracin $(50 \mu \mathrm{g} / \mathrm{ml}$ final concentration) to one half (induced sample) and leaving the other half untreated (uninduced control). After incubation for an additional $30 \mathrm{~min}$ at $37^{\circ} \mathrm{C}$ with aeration, $2 \mathrm{ml}$ of each culture were harvested and the cell pellets were frozen and kept at $-20^{\circ} \mathrm{C}$. The pellets were resuspended in $1 \mathrm{ml}$ of working buffer and assayed for $\beta$-galactosidase activity as described with normalization to cell density (Miller, 1972).

\section{Preparation of total RNA for quantitative real-time RT-PCR, Northern blots and} primer extension analysis. Total RNA was extracted from $10 \mathrm{ml}$ of culture with and without bacitracin $\left(50 \mu \mathrm{g} / \mathrm{ml}\right.$ final concentration). Bacitracin was added to the culture at $\mathrm{OD}_{600}$ of 0.4 (mid-log phase) and the cultures were incubated for $10 \mathrm{~min}$ at $37^{\circ} \mathrm{C}$ with aeration before the cells were harvested and rapidly frozen at $-70^{\circ} \mathrm{C}$. RNA was prepared using the RNeasy kit (Qiagen) according to the manufacturer's protocol. The RNA was treated with DNase (using the on-column RNase-free DNase kit from Qiagen) to remove remaining traces of chromosomal DNA that would interfere with the subsequent reaction. The success of this treatment was verified by a lack of product in a standard PCR reaction, using the same primers as for the real-time RT-PCR.

Quantitative real-time RT-PCR. Measurement of transcript abundance was performed by quantitative real-time RT-PCR, using the QuantiTect SYBRgreen RT-PCR kit (Qiagen) according to the manufacturer's procedure, with minor modifications: In brief, $100 \mathrm{ng}$ of DNA-free total RNA was used in a total reaction volume of $25 \mu 1$ with $0,5 \mu \mathrm{M}$ of each primer (see Table 2.3). The amplification reaction was carried out in an iCycler (BioRad), using the following program: initial incubation $50.0^{\circ} \mathrm{C}$ for $30 \mathrm{~min}$, followed by a $95^{\circ} \mathrm{C}$ denaturing step for $15 \mathrm{~min}$, followed by 40 cycles $\left(94^{\circ} \mathrm{C}(15 \mathrm{sec}), 60^{\circ} \mathrm{C}(30 \mathrm{sec}), 72^{\circ} \mathrm{C}(30 \mathrm{sec})\right)$. After a subsequent incubation step $\left(55^{\circ} \mathrm{C}\right.$ for $\left.1 \mathrm{~min}\right)$, the setpoint temperature was increased in 80 cycles $\left(10 \mathrm{sec}\right.$ each) by $0.5^{\circ} \mathrm{C} / \mathrm{cycle}$, starting from $55^{\circ} \mathrm{C}$. Expression of $r p s E$ and $r p s J$, encoding ribosomal proteins, was monitored as constitutive reference. Expression of liaR was calculated as fold-change based on the $\mathrm{C}_{\mathrm{T}}$ values for each gene, as described (Talaat et al., 2002). 
Table 2.1. Strains used in this study

\begin{tabular}{lll}
\hline Strain & Genotype or characteristics & Reference or source \\
\hline
\end{tabular}

\section{E. coli strains}

DH5 $\alpha \mathrm{F}^{\prime} \quad \mathrm{F}^{\prime} /$ endAl hsdR17 $\left(\mathrm{r}_{\mathrm{K}}{ }^{-}, \mathrm{m}_{\mathrm{K}}{ }^{+}\right) \operatorname{gln} \mathrm{V} 44$ thi-1 recAl gyrA $\left(\mathrm{Nal}^{\mathrm{r}}\right)$

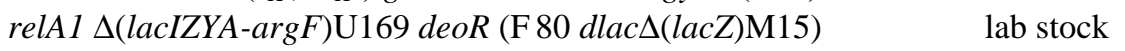

\section{B. subtilis strains}

\section{W168 wild type, $\operatorname{trp} C 2$}

CU1065 wild type, $\operatorname{trpC2,attSP\beta }$

HB0920 CU1065 liaH::kan

HB0933 CU1065 liaR::kan

HB0950

TMB002

TMB003

TMB004

TMB016

TMB017

TMB018

TMB019

TMB020

TMB021

TMB027

TMB028

TMB029

TMB053

TMB066

TMB069

TMB071

TMB072

TMB095

TMB096

TMB097

TMB098

TMB099

TMB100

TMB101

TMB102

TMB104

TMB107

TMB108

TMB111

TMB112

TMB113

TMB114

TMB115

TMB132

TMB133

TMB182

TMB183

TMB184

CU1065 liaF::kan

CU1065 liaG::kan

CU1065 liaS::kan
CU1065 attSP $\beta 2 \Delta 2:: \mathrm{P}_{\text {lial }}$-cat-lacZ

W168 amyE::(cat, $\mathrm{P}_{\text {lial }}$ lacZ)

W168 amyE::(cat, $\mathrm{P}_{\text {lial }}$-lacZ), liaG::kan

W168 amyE::(cat, $\mathrm{P}_{\text {lial }}$-lacZ), liaF::kan

W168 amyE::(cat, $\mathrm{P}_{\text {lial }}$ lacZ), liaS::kan

W168 amyE::(cat, $\mathrm{P}_{\text {lial }}$-lacZ), liaR::kan

W168 amyE::(cat, $\mathrm{P}_{\text {lial }}$-lacZ), liaGF::kan

$\mathrm{HB} 0950 \mathrm{LiaF} \Delta\left(\mathrm{I}_{151}-\mathrm{D}_{235}\right)^{2}$

HB0950 LiaF $\Delta\left(\mathrm{S}_{189^{-}}-\mathrm{V}_{192}\right)^{2}$

HB0950 LiaF $\Delta\left(\mathrm{E}_{126}-\mathrm{D}_{146}\right)^{2}$

W168 amyE: (cat, $\mathrm{P}_{l i a G(-68 \text { - 914)-lacZ) }}$

W168 amyE::(cat, $P_{y h c Y(-129-70)-l a c Z), ~ l i a R:: s p e c}$

W168 amyE::(cat, $\mathrm{P}_{y h c Y(-129-70)}$-lacZ), liaHGF::kan, liaR::spec

$\mathrm{W} 168$ amyE::(cat, $\mathrm{P}_{y h c Y(-129-70)-l a c Z)}$

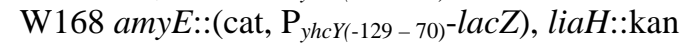

$\mathrm{W} 168$ amyE::(cat, $\mathrm{P}_{y h c Y(-129-70)-l a c Z), ~ l i a F:: k a n}$

W168 amyE::(cat, $\mathrm{P}_{y h c Y(-71-70)-l a c Z), ~ l i a H G F:: k a n}$

$\mathrm{W} 168$ amyE::(cat, $\mathrm{P}_{y h c Y(-88-70)}$-lacZ), liaHGF::kan

$\mathrm{W} 168$ amyE::(cat, $\mathrm{P}_{y h c Y(-97-70)}$-lacZ), liaHGF::kan

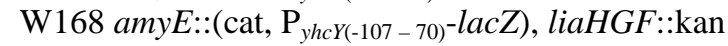

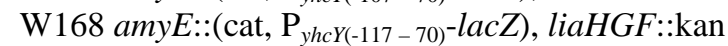

W168 amyE:(cat, $\mathrm{P}_{y h c Y(-122-70)}$ lacZ), liaHGF::kan

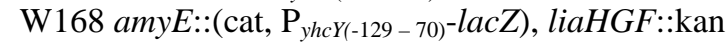

$\mathrm{W} 168$ amyE::(cat, $\mathrm{P}_{y h c Y(-244-70)}$-lacZ), liaHGF::kan

$\mathrm{W} 168$ amyE::(cat, $\mathrm{P}_{\text {liaG }(-68-3)-l a c Z)}$

$\mathrm{W} 168$ amyE::(cat, $\mathrm{P}_{\text {lial }}$-lacZ), liaH::kan

$\mathrm{W} 168$ amyE::(cat, $\left.\mathrm{P}_{\text {lial }(-102-72)}\left(\mathrm{A}_{-76} \mathrm{~T}, \mathrm{~A}_{-78} \mathrm{~T}, \mathrm{~A}_{-81} \mathrm{~T}\right)-l a c Z\right)$

$\mathrm{W} 168$ amyE::(cat, $\left.\mathrm{P}_{\text {lial }(-102-72)}\left(\mathrm{A}_{-75} \mathrm{~T}, \mathrm{~A}_{-77} \mathrm{~T}, \mathrm{~A}_{-79} \mathrm{~T}\right)-l a c Z\right)$

W168 amyE::(cat, $\left.\mathrm{P}_{\text {lial }\left(-102-{ }_{72}\right)}\left(\mathrm{C}_{-86} \mathrm{~A}, \mathrm{G}_{-88} \mathrm{~A}, \mathrm{C}_{-93} \mathrm{~A}, \mathrm{G}_{-95} \mathrm{~A}, \mathrm{~T}_{-98} \mathrm{~A}\right)-l a c Z\right)$ $\mathrm{W} 168$ amyE::(cat, $\left.\mathrm{P}_{\text {lial }(-102-72)}\left(\mathrm{A}_{-78} \mathrm{C}, \mathrm{A}_{-83} \mathrm{C}\right)-l a c Z\right)$

W168 amyE::(cat, $\mathrm{P}_{\text {lial }\left(-102-{ }_{72}\right)}\left(\mathrm{C}_{-93} \mathrm{~A}, \mathrm{G}_{-95} \mathrm{~A}, \mathrm{~T}_{-98} \mathrm{~A}\right)-$ lacZ)

$\mathrm{W} 168$ amyE::(cat, $\mathrm{P}_{\text {liaG }(81-914)}$-lacZ)

$\mathrm{W} 168$ amyE::(cat, $\mathrm{P}_{\text {lial }\left(-102-7_{2}\right)}\left(\mathrm{C}_{-86} \mathrm{~A}, \mathrm{~T}_{-87} \mathrm{~A}, \mathrm{G}_{-88} \mathrm{~A}\right)$-lacZ)

HB0950 LiaF $\Delta\left(\mathrm{I}_{151}-\mathrm{D}_{235}\right)^{2}$, thrC::(spec, $\mathrm{P}_{x y l}$-liaF $)$

HB0950 LiaF $\Delta\left(\mathrm{S}_{189^{-}}-\mathrm{V}_{192}\right)^{2}$, thrC::(spec, $\mathrm{P}_{x y l}$-liaF $)$

HB0950 LiaF $\Delta\left(\mathrm{E}_{126}-\mathrm{D}_{146}\right)^{2}$, thrC::(spec, $\mathrm{P}_{x y l}$-liaF $)$ lab stock

lab stock

(Mascher et al., 2003)

(Mascher et al., 2003)

(Mascher et al., 2004)

this study

this study

this study

this study

this study

this study

this study

this study

this study

this study

this study

this study

this study

this study

this study

this study

this study

this study

this study

this study

this study

this study

this study

this study

this study

this study

this study

this study

this study

this study

this study

this study

this study

this study

this study

this study

this study

this study

${ }^{1}$ resistance cassettes: $\mathrm{kan}=$ kanamycin; $\mathrm{cat}=$ chloramphenicol; $\mathrm{spec}=$ spectinomycin. Positions of promoter fragments are given relative to the "ATG" start codon of the corresponding genes.

${ }^{2}$ For reasons of clarity, the effect of in-frame deletions in liaF are given at the level of the LiaF protein. 
Table 2.2. Vectors and plasmids

\begin{tabular}{|c|c|c|c|}
\hline Plasmid & $\begin{array}{l}\text { Genotype, sequence or characteristics }{ }^{1} \\
\text { for cloning }\end{array}$ & $\begin{array}{l}\text { Primers used } \\
\text { or source }\end{array}$ & Reference \\
\hline pAC6 & $\begin{array}{l}\text { lacZ fusion vector, integrates at } a m y E \text {, chloramphenicol } \\
\text { resistance }\end{array}$ & & (Stülke et al., 1997) \\
\hline pXT & $\begin{array}{l}\text { vector for xylose-inducible gene expression, integrates at } \\
\text { thrC, pDG1782-derivative, spectinomycin resistance }\end{array}$ & & (Derre et al., 2000) \\
\hline pAJ601 & pAC6 $\mathrm{P}_{v h c Y(-71-70)-l a c Z}$ & \#0165/\#0172 & this study \\
\hline pAJ602 & pAC6 $\mathrm{P}_{y h c Y(-88-70)-l a c Z}$ & \#0165/\#0171 & this study \\
\hline pAJ603 & pAC6 $\mathrm{P}_{y h c Y(-129-70)-l a c Z}$ & $\# 0165 / \# 0168$ & this study \\
\hline pAJ604 & pAC6 $\mathrm{P}_{y h c Y(-97-70)-l a c Z}$ & \#0165/\#0170 & this study \\
\hline pAJ606 & pAC6 $\mathrm{P}_{\mathrm{yhc}(\mathrm{Y}-244-70)-l a c Z}$ & \#0165/\#0166 & this study \\
\hline pAJ607 & pAC6 $\mathrm{P}_{y h c Y(-122-70)-l a c Z}$ & $\# 0165 / \# 0169$ & this study \\
\hline pAJ608 & pAC6 $\mathrm{P}_{y h c Y(-117-70)-l a c Z}$ & \#0165/\#0259 & this study \\
\hline pAJ609 & pAC6 $\mathrm{P}_{y h c Y(-107-70)-l a c Z}$ & \#0165/\#0260 & this study \\
\hline pBD601 & pAC6 $\mathrm{P}_{\text {lial }(-102-72)}\left(\mathrm{A}_{-76} \mathrm{~T}, \mathrm{~A}_{-78} \mathrm{~T}, \mathrm{~A}_{-81} \mathrm{~T}\right)-l a c \mathrm{Z}$ & \#0100/\#0231 & this study \\
\hline pBD602 & pAC6 $\mathrm{P}_{\text {lial }(-102-72)}\left(\mathrm{A}_{-75} \mathrm{~T}, \mathrm{~A}_{-77} \mathrm{~T}, \mathrm{~A}_{-79} \mathrm{~T}\right)-l a c Z$ & $\# 0100 / \# 0232$ & this study \\
\hline pBD603 & pAC6 $\mathrm{P}_{\text {lial }(-102-72)}\left(\mathrm{C}_{-86} \mathrm{~A}, \mathrm{G}_{-88} \mathrm{~A}, \mathrm{C}_{-93} \mathrm{~A}, \mathrm{G}_{-95} \mathrm{~A}, \mathrm{~T}_{-98} \mathrm{~A}\right)-l a c \mathrm{Z}$ & \#0100/\#0265 & this study \\
\hline pBD604 & pAC6 $\mathrm{P}_{\text {lial }(-102-72)}\left(\mathrm{A}_{-78} \mathrm{C}, \mathrm{A}_{-83} \mathrm{C}\right)-\mathrm{lacZ}$ & \#0100/\#0266 & this study \\
\hline pBD605 & pAC6 $\mathrm{P}_{\text {lial }(-102-72)}\left(\mathrm{C}_{-93} \mathrm{~A}, \mathrm{G}_{-95} \mathrm{~A}, \mathrm{~T}_{-98} \mathrm{~A}\right)-$ lac & $\# 0100 / \# 0267$ & this study \\
\hline pBD606 & pAC6 $\mathrm{P}_{\text {lial }(-102-72)}\left(\mathrm{C}_{-86} \mathrm{~A}, \mathrm{~T}_{-87} \mathrm{~A}, \mathrm{G}_{-88} \mathrm{~A}\right)-$-lacZ & \#0100/\#0268 & this study \\
\hline pDH605 & pAC6 $\mathrm{P}_{\text {liaG }(81-914)-l a c Z}$ & \#0204/\#0222 & this study \\
\hline pSJ601 & pAC6 $\mathrm{P}_{\text {liaG(-68 - 914) }}-\mathrm{lac} Z$ & \#0204/\#0205 & this study \\
\hline pSJ607 & pAC6 $\mathrm{P}_{l i a G(-68-3)^{-l a c Z}}$ & \#0204/\#0296 & this study \\
\hline pSJ701 & pXT $\mathrm{P}_{x y l}$-liaF & \#0035/\#0036 & this study \\
\hline pTM1 & pAC6 $\mathrm{P}_{\text {lial( }-83-72)-l a c Z}$ & \#0099/\#0100 & this study \\
\hline
\end{tabular}

${ }^{1}$ The positions of the cloned fragments are given relative to the " $\mathrm{A}$ " of the start codon of the corresponding genes.

Primer extension mapping of the $y h c Y$ transcriptional start site. For mapping of the $y h c Y$ promoter, HB0920 cells were grown in LB and total RNA was isolated from uninduced and bacitracin-induced (final concentration $50 \mu \mathrm{g} / \mathrm{ml}$ ) mid-logarithmic cultures as described above. Primer extension reactions for $y h c Y$ were set up as follows: $30 \mu \mathrm{g}$ of heat-denatured RNA was hybridized at $65^{\circ} \mathrm{C}$ to $\sim 2$ pmol of end-labeled primer yhcY-PE (Table 2.3) in buffer containing $60 \mathrm{mM} \mathrm{NaCl}, 50 \mathrm{mM}$ Tris- $\mathrm{HCl}(\mathrm{pH}$ 7.9), $10 \mathrm{mM}$ DTT, and $40 \mathrm{U}$ of RNasin (Promega) in a total volume of $30 \mu$ l. Following hybridization, $50 \mu$ l extension buffer $(72 \mathrm{mM}$ $\mathrm{NaCl}, 50 \mathrm{mM}$ Tris-HCl [pH 7.9], $10 \mathrm{mM}$ DTT, $20 \mathrm{mM} \mathrm{MgCl}$ ), dNTPs (10 mM), and $2 \mu \mathrm{l}$ Superscript II reverse transcriptase (Invitrogen) were added to the mixture and incubation continued at $37^{\circ} \mathrm{C}$ for $30 \mathrm{~min}$. The primer extension products were precipitated with ethanol, resuspended in sequence loading buffer, and loaded onto a $6 \%$ polyacrylamide sequencing gel. A PCR cycle sequencing kit (Epicentre) was used to generate sequencing ladders corresponding to the $y h c Y$ promoter region. 
Table 2.3. Oligonucleotides used in this study

Primer-number or Sequence
amplified fragment

\begin{tabular}{|c|c|}
\hline \multicolumn{2}{|c|}{ Oligonucleotides for Long Flanking Homology (LFH)-PCR ${ }^{1}$} \\
\hline kan cassette & fwd: CAGCGAACCATTTGAGGTGATAGG, rev: CGATACAAATTCCTCGTAGGCGCTCGG \\
\hline kan-check & fwd: CATCCGCAACTGTCCATACTCTG, rev: CTGCСТССТCАТССТСТTCATCC \\
\hline spec cassette & $\begin{array}{l}\text { fwd: CAGCGAACCATTTGAGGTGATAGGGACTGGCTCGCTAATAACGTAACGTGACTGGCAAGAG, } \\
\text { rev: }\end{array}$ \\
\hline spec-ckeck & fwd: GTTATCTTGGAGAGAATATTGAATGGAC, rev: CGTATGTATTCAAATATATCCTCCTCAC \\
\hline liaH-up & fwd: CTTGTTATTCGTCACTGCC, rev: CCTATCACCTCAAATGGTTCGCTGGTCCTTCATGAACTGACGC \\
\hline liaH-do & fwd: CGAGCGCCTACGAGGAATTTGTATCGCAGACCAGACAAAAGCGGC, rev: CGCTAGATCCCCGCTGTCC \\
\hline liaG-up & fwd: TTGTCGTCGGAATCGCATTGGC, rev: CCTATCACCTCAAATGGTTCGCTGCACATCTTTAACGACGACGGC \\
\hline liaG-do & fwd: CGAGCGCCTACGAGGAATTTGTATCGCCAATCGACATCAAAACGGACA, rev: TTACCCGGCGTTTGACTCGC \\
\hline liaF-up & fwd: AAGGATTTGCGGTCAAGTCC, rev: CCTATCACCTCAAATGGTTCGCTGGCAATGATCAATCCGAGAAGC \\
\hline liaF-do & fwd: CGAGCGCCTACGAGGAATTTGTATCGGATGTGGATGTGAAGTACG, rev: TTCAAGCCGTATGAGGAGGC \\
\hline liaS-up & fwd: GCTTTATCAGCAAGCGGTGACG, rev: CCTATCACCTCAAATGGTTCGCTGTCCCGTTGTCATGCGGATGGC \\
\hline liaS-do & fwd: CGAGCGCCTACGAGGAATTTGTATCGGGCACTCAAATCGAAGTGA, rev: AACCGGGCTGGGAAACGAGGTC \\
\hline liaR-up & fwd: GCTGTCATCAAGCTGGTTCGG, rev: CCTATCACCTCAAATGGTTCGCTGCGATGCTTCGCCGATGACTTC \\
\hline liaR-do & wd: CGAGCGCCTACGAGGAATTTGTATCGACGCACACCGAAATCATCTC, rev: CTCTTCATCTGATCCGACACAGC \\
\hline
\end{tabular}

Oligonucleotides for quantitative real-time RT-PCR, primer extension, Northern hybridization and sequencing liaR-RT fwd: ATTGAAGTCATCGGCGAAGC, rev: AAAGCTCCCGGCAAATTTGC rpsJ-RT fwd: GAAACGGCAAAACGTTCTGG, rev: GTGTTGGGTTCACAATGTCG $r p s E$-RT fwd: GCGTCGTATTGACCCAAGC, rev: TACCAGTACCGAATCCTACG $y h c Y$-PE GGTTTCCGCAATCGTTTTCAGCG yhc $Y$ probe ${ }^{2}$ fwd: GAGTTGCTGAGTCTGACAAACC, rev: CTAATACGACTCACTATAGGGAGATCGTGAAGCTCCTGAGCGAGGC liaF sequencing fwd: GCTTTATCAGCAAGCGGTGACG, rev: CCGAACCAGCTTGATGACAGC

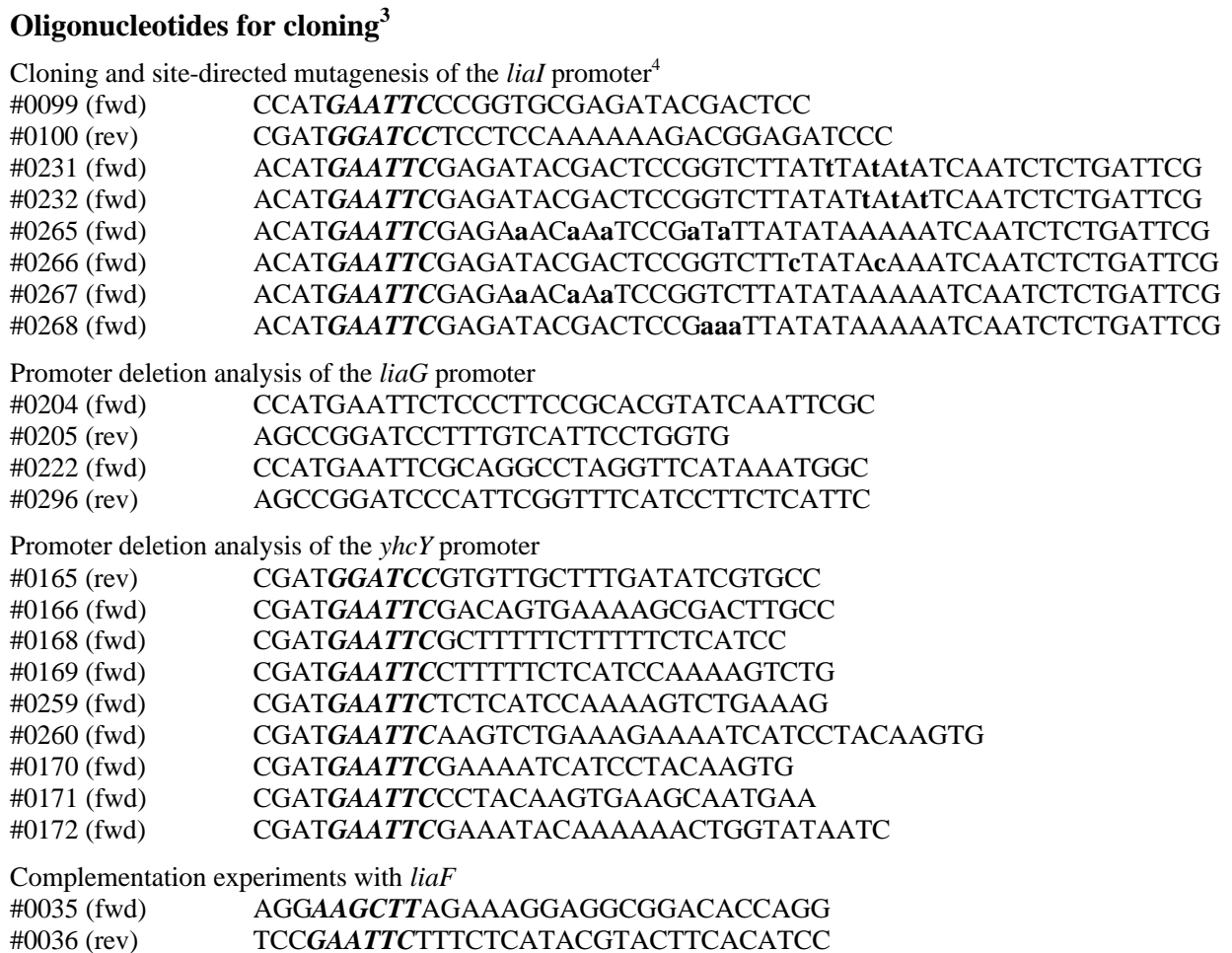

${ }^{1}$ Oligonucleotide names refer to the fragments flanking the gene to be deleted. Sequences underlined are inverse and complimentary to the 5'- (up-rev) and 3'- (do-fwd) end of the kanamycin cassette, respectively. ${ }^{2}$ The underlined sequence represents the T7 promoter necessary for the construction of RNA-probes by in-vitro transcritption ${ }^{3}$ Restriction sites for cloning are highlighted in bold italics. ${ }^{4}$ Nucleotides given in small bold letters represent mismatches. 
Comparative genomics analyses. Multiple sequence alignments were performed using ClustalW, implemented in the BioEdit program package (Hall, 1999). Domain-based analysis of protein sequences were performed using the SMART database (Schultz et al., 1998) at http://smart.embl-heidelberg.de/. The genomic context clustering analysis of the lia locus was performed using the ERGO database, which is available through Integrated Genomics, Inc. (http://www.integratedgenomics) and maintained by the GenoMIK center, Göttingen. The initial identification of the putative LiaR-binding site was done by submitting promoter fragments of 200 nucleotides upstream the ATG of all liaI homologs identified above to the MEME at http://meme.sdsc.edu/meme/website/meme.html (Bailey and Elkan, 1994). The resulting weight matrix of the conserved sequence pattern was then used to screen the individual genomes harboring liaRS homologous genes for additional putative LiaR-binding sites with the help of the virtual footprint algorithm (Münch et al., 2005), implemented into the Prodoric database (Münch et al., 2003) at http://www.prodoric.de/vfp/. The graphical representation of the putative LiaR-binding site was generated using the Weblogo interface (Crooks et al., 2004) at http://weblogo.berkeley.edu/.

\section{Results}

LiaF is an integral part of the LiaRS-mediated signal transduction system. With the exception of liaSR, encoding the sensor kinase and cognate response regulator of a classical bacterial TCS, the function of the gene products of the liaIHGFSR locus is unknown. No homology can be found in the databases for liaI and liaG. Both genes encode hypothetical proteins, harboring two or one transmembrane helices, respectively, indicative for a membrane localization. The gene product of liaH belongs to the PspA/IM30 protein family (see below), while liaF encodes a putative membrane protein with homology to proteins of unknown functions from other Gram-positive bacteria.

To investigate their possible roles in LiaRS-mediated signal transduction, we investigated the effect of various insertion-deletions in the lia locus on $\mathrm{P}_{\text {lial }}$ activity as measured from a $\mathrm{P}_{\text {lial }}{ }^{-}$ lacZ reporter fusion integrated ectopically at the $a m y E$ locus (Fig. 2.1). The wild-type strain (TMB016) showed a strong response to the presence of bacitracin, resulting in a more than 200 -fold induction of $\mathrm{P}_{\text {lial }}$ activity, while virtually no $\beta$-galactosidase activity can be detected in the liaR::kan strain (TMB020) under either inducing or non-inducing conditions, consistent with the stringent LiaR-dependence of $\mathrm{P}_{\text {lial }}$ (Mascher et al., 2004). The liaS::kan mutant 
TMB019, lacking the histidine kinase, no longer responded to bacitracin, but did display a slightly increased basal expression level. Surprisingly, $\mathrm{P}_{\text {lial }}$ was constitutively active in the liaF::kan mutant TMB018, even in the absence of the inducer. This activity was even ten-fold higher than that measured in the induced wild type.

Resistance cassettes inserted into the chromosome can exhibit polar effects on downstream genes. This can either be a positive polar effect, due to readthrough from their own strong promoters (Cao et al., 2003), or a negative effect due to termination of transcription within the resistance cassette. To investigate possible polar effects, we used quantitative real-time RTPCR to measure liaR transcription (in the insertion-deletion strains TMB017-TMB019) relative to the uninduced wild type. In all strains, there was an $\sim$ four-fold increase in liaR transcript. Since $\mathrm{P}_{\text {liaI }}$ transcription behaves identically in the wild-type and a liaG::kan strain, while it is constitutively active in the liaF::kan strain, there is no correlation between the weak positive polar effects from the kanamycin resistance cassettes and the observed effects on $\mathrm{P}_{\text {lial }}$ activity. Instead, the results support a functional role for LiaF in inhibiting LiaRSmediated signal transduction.

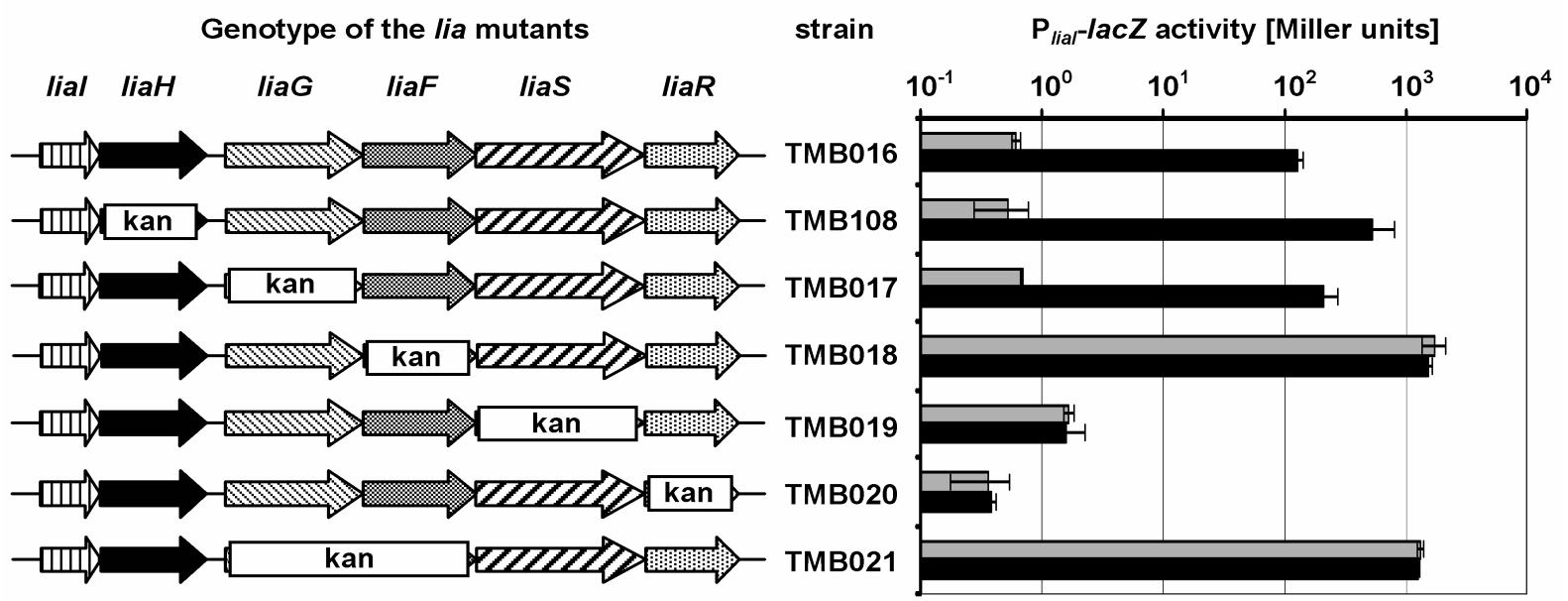

Figure 2.1. $\mathbf{P}_{\text {liaI }}$ activity in response to deletions of lia genes. Cultures of strains TMB016 ("wild type") and TMB017-TMB020/TMB108 were grown in LB medium to mid-log phase $\left(\mathrm{OD}_{600} \sim 0.4\right)$ and split. One half was induced by the addition of bacitracin (final concentration $50 \mu \mathrm{g} / \mathrm{ml}$; black bars), the other half served as an uninduced control (grey bars). Cells were harvested $30 \mathrm{~min}$ post-induction and assayed as described previously (Mascher et al., 2004). $\beta$-Galactosidase activity is expressed in Miller units (Miller, 1972). A log scale was applied for reasons of clarity. The genotype of the corresponding strains is shown on the left side (see Fig. 3A for labeling pattern of arrows). "kan" represents the area replaced by the kanamycin resistance cassette. The cassette itself has a size of approx. $1.5 \mathrm{~kb}$ in all mutants.

Spontaneous in-frame deletions in liaF lead to constitutive activity of $\mathbf{P}_{\text {lial }}$. The strong effect of a liaF deletion on $\mathrm{P}_{\text {lial }}$ prompted us to perform a genetic screen using strain HB0950, which carries an ectopic pJPM122-based $\mathrm{P}_{\text {lial }}$-cat-lacZ fusion integrated at attSP $\beta$ (Mascher $e t$ al., 2004). Direct plating of a mid-logarithmic culture of HB0950 on LB plates with 
chloramphenicol gave rise to numerous $\mathrm{Cm}^{\mathrm{R}}$ colonies. We reasoned that any mutation in liaF that renders its gene product dysfunctional would lead to such a spontaneous chloramphenicol resistance in the genetic background of HB0950. Indeed, PCR and sequence analyses indicate that alterations in liaF were associated with many of the spontaneous $\mathrm{Cm}^{\mathrm{R}}$ mutants. Three strains containing in-frame deletions in lia $F$ were chosen for further analysis (Fig. 2.2). These deletions likely arose from recombination between repeated nucleotide sequences (Fig. 2.2a, upper line) - as has been suggested for a comparable deletion in the Enterococcus faecium histidine kinase VanSB (Depardieu et al., 2003) - and result in the deletion of 84, 20, and 4 amino acids. All three in-frame deletion mutants showed a strong bacitracin-independent, constitutive upregulation of LacZ expression in B-galactosidase assays (Fig. 2.3), a behavior similar to TMB018 (liaF::kan) (Fig. 2.1). The effect of these mutations could be complemented by re-introduction of a wild type liaF allel, integrated as single copy into the thrC-locus and expressed from a xylose-inducible promoter (see Material and Methods): In the resulting strains, TMB182-184 (Table 2.1), $\mathrm{P}_{\text {lial }}$ is again switched off in the absence and inducible by addition of bacitracin (data not shown). Taken together, these data clearly demonstrate a negative role for the putative transmembrane protein LiaF in LiaRS-mediated signal transduction and identify regions in the hydrophilic C-terminus that are crucial for LiaF function.

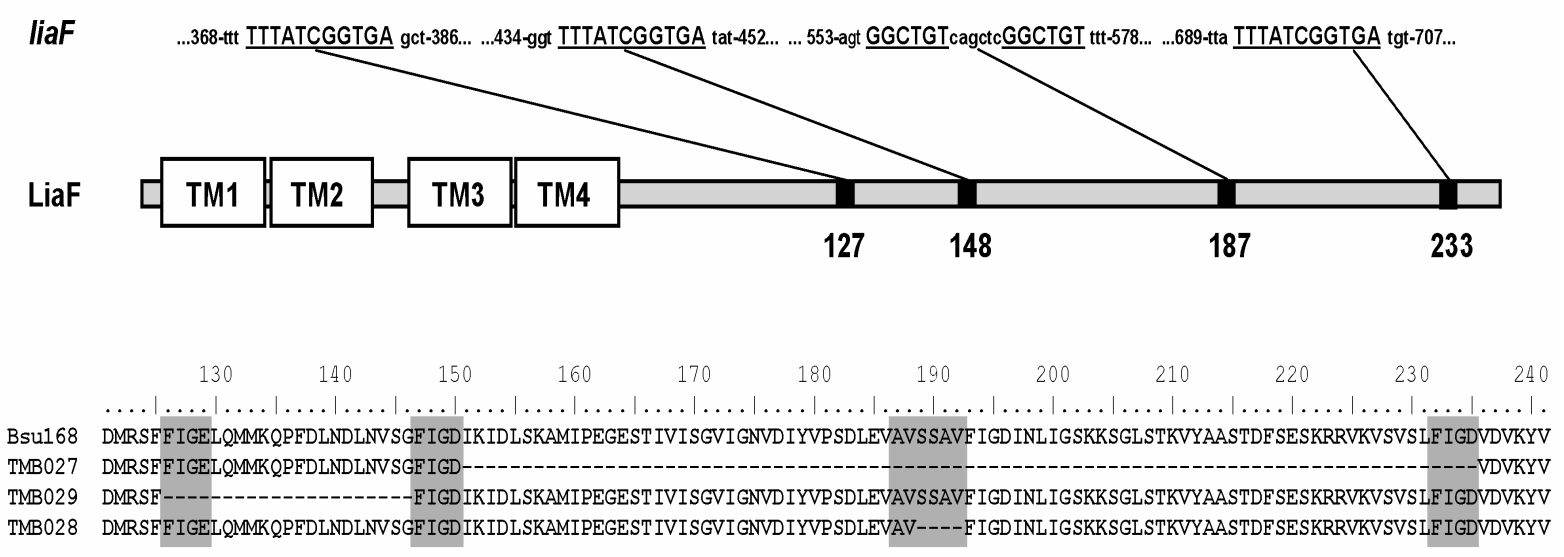

Figure 2.2. Features and sequence of LiaF-derivatives in spontaneous chloramphenicol resistant mutants, based on in-frame deletions. A sequence alignment of the C-terminal half of LiaF is shown in the lower part, including the reference sequence of the wild type strain W168 in the first line. The amino acids corresponding to the 11 or 6 nucleotide repeat (underlined in the upper part for the liaF gene) are shaded in grey. A graphical representation of LiaF is shown in the middle. The four putative transmembrane helices (TM1-4) are located in the N-terminal half of LiaF. The black boxes indicate the positions of the four repeats. 


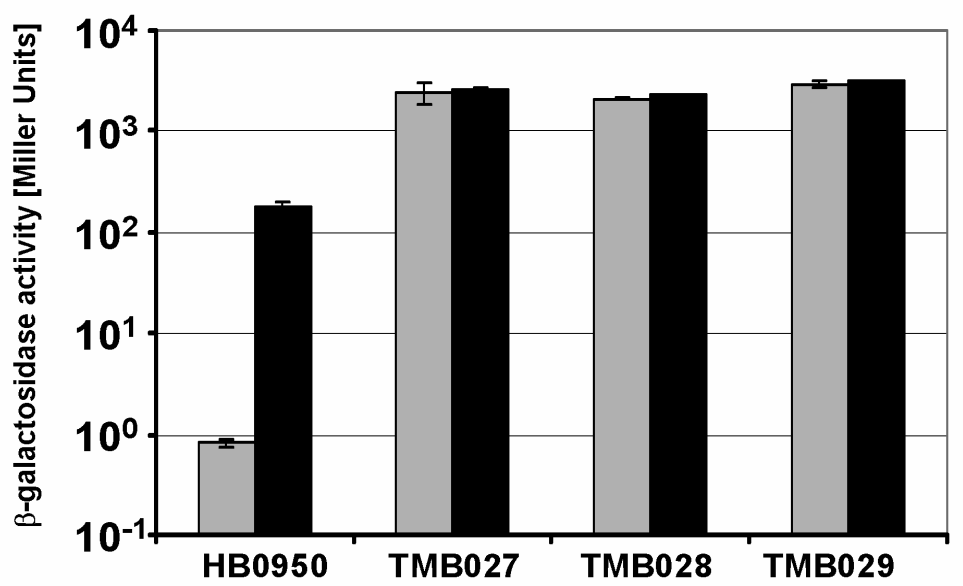

Figure 2.3. $\mathbf{P}_{\text {liaI }}$ activity in $\mathrm{HB} 0950$ ("wild type") and derived mutants harboring in-frame deletion in liaF. Experimental conditions and labeling of the bars as described in Fig. 2.1.

The genomic context of liaFSR is conserved in the Firmicutes group of Gram-positive bacteria. A functional connection between proteins is very often reflected by a clustering of their respective genes at the genomic level. For example, a functional connection has been demonstrated for some TCS involved in cell envelope stress response in the Bacillus/Clostridium group of bacteria which cluster with operons encoding ABC-transporters (Joseph et al., 2002). In these detoxification units, the TCS senses the presence of a harmful compound and strongly induces the expression of the corresponding $\mathrm{ABC}$ transporter, which then facilitates removal (Mascher et al., 2003; Neumüller et al., 2001; Ohki et al., 2003a).

A genomic context clustering analysis of LiaS orthologs revealed a topological conservation of all three genes, liaFSR, in Gram-positive bacteria with a low $\mathrm{G}+\mathrm{C}$ content. Without exception, a liaF homolog is always located directly upstream of the TCS in all species harboring liaSR-homologs (Fig. 2.4). This finding further substantiates the functional link between LiaF and LiaRS. In contrast, homologs of lialH are only present in the lia locus of bacilli. Both genes form a separate operon in Listeria species, and are apparently lacking in the more distantly related cocci. The liaG gene is only found in Bacillus licheniformis and B. halodurans, the bacteria most closely related to B. subtilis for which complete genome sequences are available (Fig. 2.4).

Identification of a conserved promoter element as a putative binding-site for LiaRhomologous response regulators from the genus Bacillus. Protein sequence comparisons (Hall, 1999) revealed an unusually high degree of sequence similarity in the C-terminal domain of all LiaR-homologs (data not shown). This domain harbors a LuxR-like helix-turnhelix motif and defines the DNA-binding specificity of LiaR-like response regulators. We 


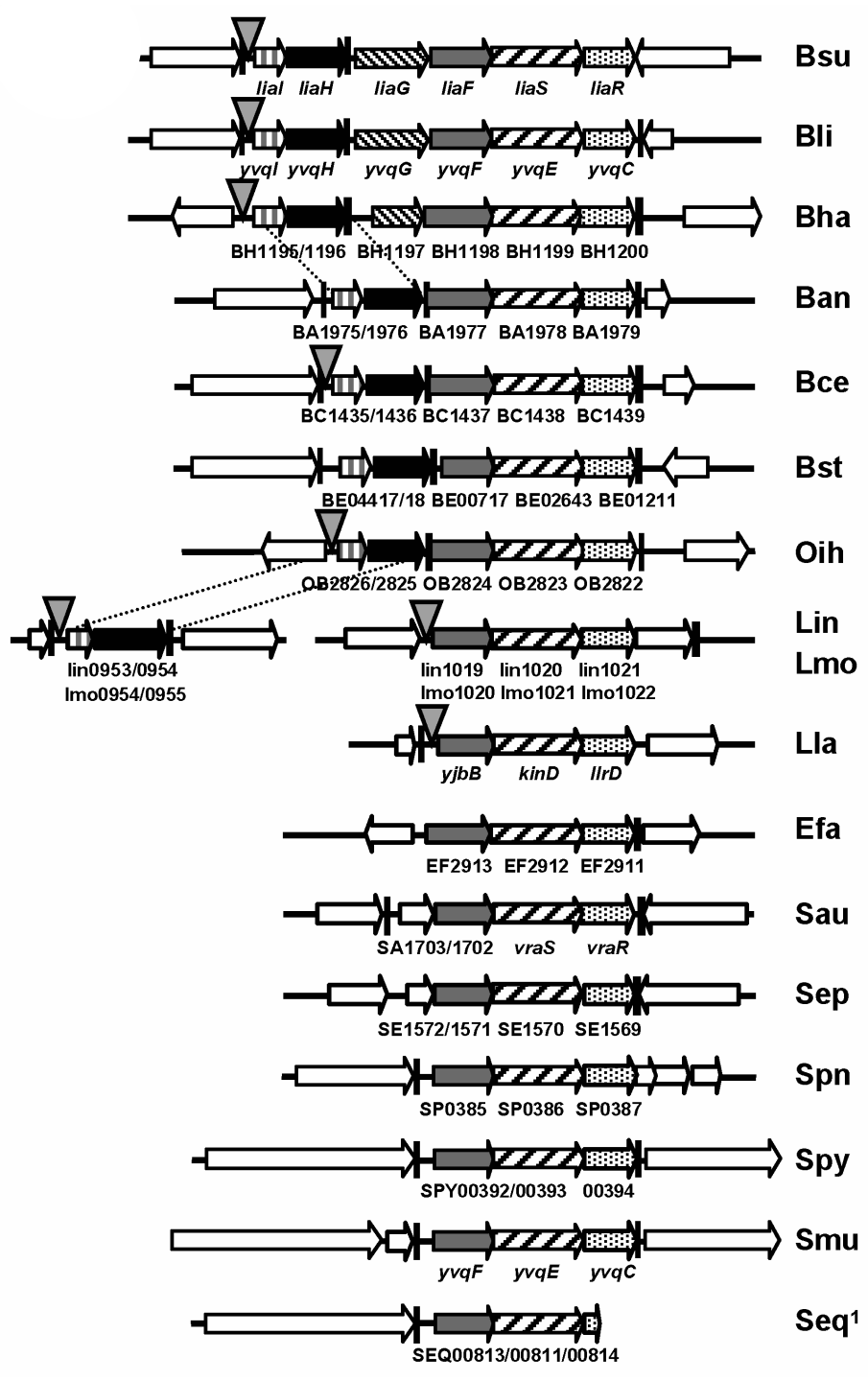

Figure 2.4. Comparative genomics analysis of the lia locus and identification of the putative LiaR-binding site. Conservation of the lia locus in other Gram-positive bacteria with a low $\mathrm{G}+\mathrm{C}$ content. The loci are drawn to scale, with the line of the B. subtilis lia-locus corresponding to $7.5 \mathrm{~kb}$. The genes of the lia-locus are labeled differently for clarity. Hatched arrows represent genes coding for histidine kinases and dotted arrows response regulators, homologous to LiaS and LiaR, respectively. The liaF homologs are shown as grey, liaH as black arrows and liaI as white arrows with black vertical lines; genes flanking the lia-locus are represented by white arrows. Putative terminators are marked as black vertical bars. The presence of putative LiaR-binding sites is indicated by the grey triangles (see Table 2.5 for details). Gene names according to GenBank entries of the published genome sequences. Abbreviations of bacterial species: Bsu (Bacillus subtilis), Bli (B. licheniformis) Bha (B. halodurans), Ban (B. anthracis), Bce (B. cereus), Bst (B. stearothermophilus), Oih (Oceanobacillus iheyensis), Lin (Listeria innocua), Lmo (L. monocytogenes), Efa (Enterococcus faecalis), Sau (Staphylococcus aureus), Sep (S. epidermidis), Spn (Streptococcus pneumoniae), Spy (S. pyogenes), Smu (S. mutans), Seq $(S$. equi), Lla (Lactococcus lactis). ${ }^{1}$ The genome of $S$. equi is not yet finished. The end of a contig lies inside the liaR homolog SEQ00814 
reasoned that this finding could be indicative for a conservation of the corresponding DNAbinding site within LiaR-target promoters.

To test this hypothesis, we retrieved DNA sequences upstream of liaI-homologous genes from the first nine bacterial species shown in Fig. 2.4, assuming that these promoters are all subject to regulation by their corresponding LiaRS-homologs. The sequences were submitted to the MEME web page (Bailey and Elkan, 1994), to identify short stretches of high sequence similarity. One motif was present in all but two regions $(B$. anthracis and $B$. stearothermophilus) at a similar distance relative to the start codon. This motif includes an imperfect inverted repeat of seven nucleotides, separated by two base pairs (Fig. 2.5). The resulting weight matrix of this sequence pattern was subsequently submitted to the 'Virtual Footprint' algorithm (Münch et al., 2005), implemented in the Prodoric database (Münch et $a l ., 2003)$, in order to identify candidate LiaR target promoters in the B. subtilis genome. We retrieved three sequences within intergenic regions.

In addition to the $\mathrm{P}_{\text {lial }}$ promoter region, two new potential LiaR-binding sites were identified. The first is associated with the $y h c Y Z-y h d A$ operon, a locus previously implicated as part of the LiaR regulon (Mascher et al., 2003). The second putative LiaR-box was located in a large non-coding region between the genes yozJ and rapK. Subsequent analyses, using reporter fusions failed to reveal any transcriptional activity in either direction from this intergenic region (data not shown). Thus, this putative LiaR-binding site may be a remnant of a previously functional locus, or a false-positive generated by our search algorithm.

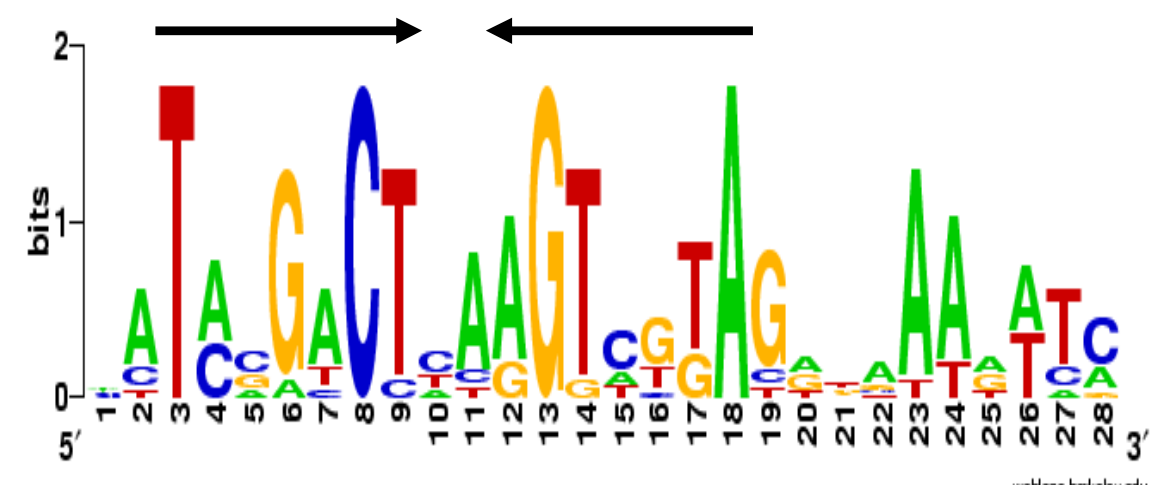

Figure 2.5. Graphical representation of the putative LiaR-binding site upstream $\mathbf{P}_{\text {lial }}$. The sequence is derived from a comparative genomics analysis (using the MEME algorithm) (Bailey and Elkan, 1994), based on the promoter regions upstream of liaI and its homologs in bacteria harboring homologous genes to both, liaRS and liaI. This graphical representation was generated through the Weblogo page (Crooks et al., 2004) at http://weblogo.berkeley.edu/logo.cgi. 
The yhcYZ-yhdA operon is expressed from a LiaR-dependent promoter. A regulatory link between the $y h c Y Z-y h d A$ operon and the Lia system had been suggested previously: bacitracin treatment leads to increased expression of this operon in a liaH mutant relative to the wild type (Mascher et al., 2003). Additionally, a LiaR-dependent expression of the $y h c Y Z-y h d A$ operon was described in a comprehensive microarray study on TCS in B. subtilis: Overexpression of LiaR, in the absence of its cognate histidine kinase LiaS, resulted in an induction of this operon (Kobayashi et al., 2001). However, these experiments do not discriminate between direct and indirect effects of LiaR on the $y h c Y Z-y h d A$ operon.

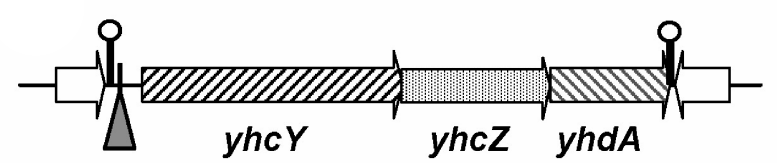

Figure 2.6. Genomic context of the putative LiaR binding sites upstream the yhcYZ-yhdA operon. The region is drawn to scale, representing $3000 \mathrm{bp}$. The exact position of the LiaR-binding site is indicated by the grey triangle. Labeling of arrows as in Fig. 2.4.

To determine if $y h c Y Z-y h d A$ is regulated by the LiaFRS system, we constructed strains carrying an ectopically integrated $\mathrm{P}_{y h c} \mathrm{Y}$-lacZ fusion. Consistent with previous transcriptome analyses (Mascher et al., 2003), $\mathrm{P}_{y h c Y}$ was only weakly inducible by bacitracin in the wildtype and was derepressed in the liaH mutant TMB072 (Fig. 2.7). While the magnitude of induction was relatively weak compared to that observed with $\mathrm{P}_{\text {lial }}$ (Fig. 2.1), induction was completely LiaR-dependent and expression was constitutive in the liaF mutant (TMB095). Since the gene products of both liaH and liaF exhibited a negative effect on $\mathrm{P}_{y h c Y}$ activity, we also introduced a liaHGF::kan insertion-deletion into TMB071 (resulting in strain TMB102). The mutant behaved like the liaF mutant, consistent with the idea that LiaR is already completely activated in a liaF mutant. As expected, both the liaH and liaF effects are completely LiaR-dependent (Fig. 2.7A). The behavior of $\mathrm{P}_{y h c Y}$ is comparable to $\mathrm{P}_{\text {lial }}$, but the activity as well as the range of inducibility is lower. This weaker activity may be due to a poorer match between the LiaR box preceeding $y h c Y$ compared to that preceeding liaI (Table 2.5).

We mapped the 5'-end of the $y h c Y$ transcript by primer extension to an "A" 41 nucleotides upstream of the start codon, thereby also verifying the induction of transcription by bacitracin in a liaH mutant (Fig. 2.7B). The $y h c Y$ promoter has a well conserved extended -10 region (TGgTATAAT), but a poorly conserved -35 region (GTGAAG) (Fig. 2.7D). Northern 
(A) Genotype of the lia mutants

strain $P_{\text {yhcr-lacZ activity [Miller units] }}$
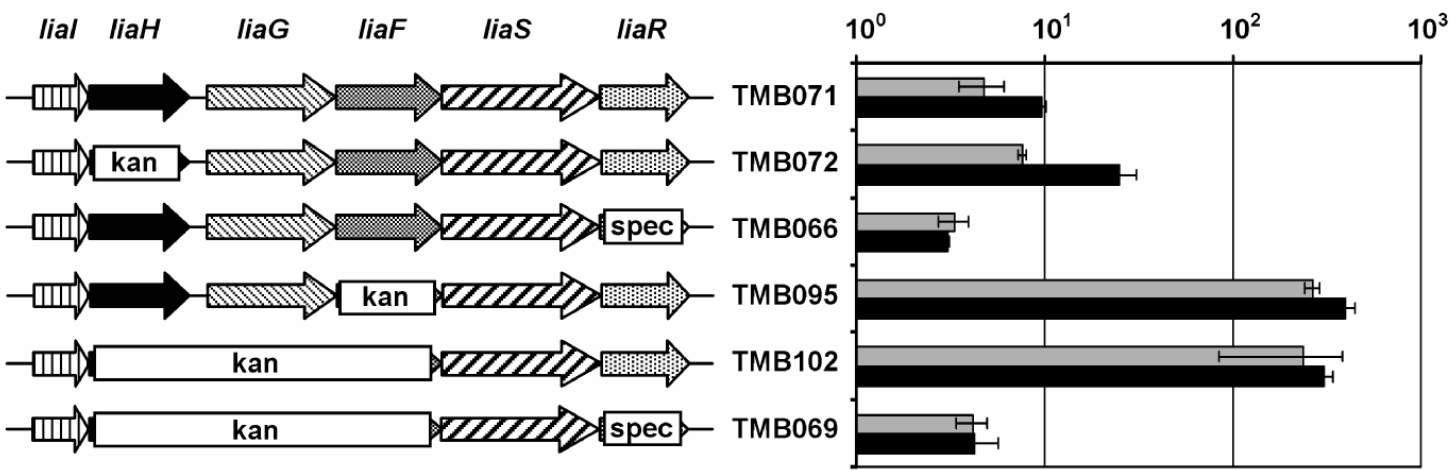

(B)

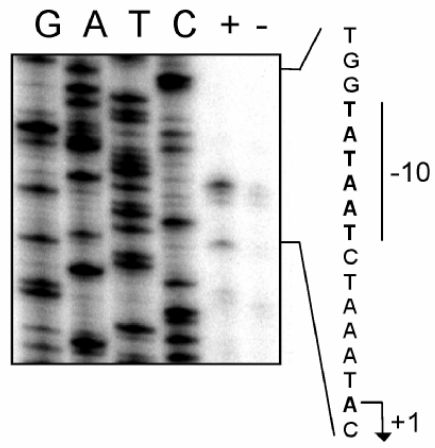

(C)

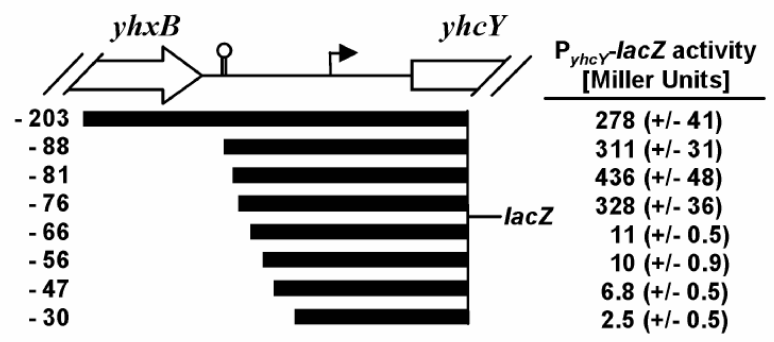

(D)

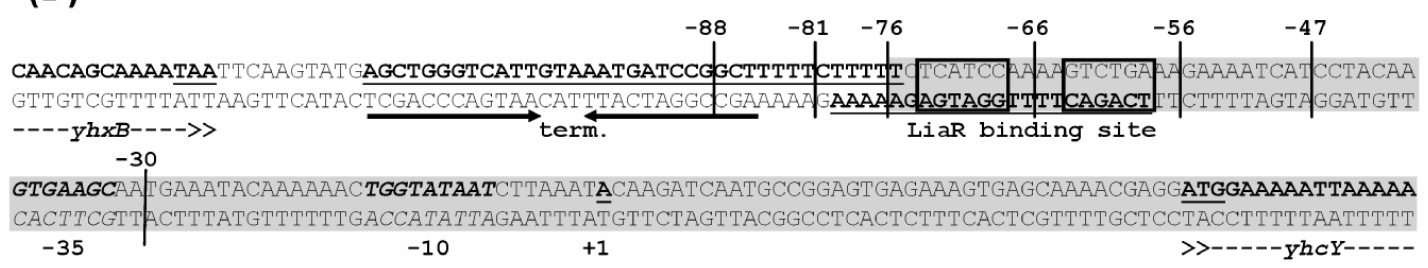

Figure 2.7. Characterization of the $y h c Y$ promoter and its LiaR-dependent expression. (A) $\mathrm{P}_{y h c Y^{-}} l a c Z$ in response to deletions of lia genes, determined by $\beta$-galactosidase assay with and without the addition of bacitracin, essentially as described. See legend to Fig. 1 for further details. A logarithmic representation of the resulting $\beta$-galactosidase activity was chosen for reasons of clarity. (B) Mapping of the transcriptional start site by primer extension analysis indicates that transcription initiates with a " $\mathrm{A}$ " as shown in the sequence to the right. Primer extension reaction was performed with RNA prepared from strain HB0920 with and without the addition of bacitracin, using the primer yhcY-PE (see Table 3). The sequencing ladder to the left was generated using the same primer, following standard procedures. (C) Promoter deletion analysis of $\mathrm{P}_{y h c Y}$. A graphical representation of the intergenic region and outline of the fragments used for the promoter dissection are shown on the left. The end of $y h x B$ and the beginning of $y h c Y$ are labeled. The putative rho-independent terminator downstream of $y h x B$ is indicated by the black stem-loop symbol. The arrow indicates the transcriptional start site. The distance of the 5'-end of the cloned fragments from the transcriptional start is indicated. The activity of these promoters was measured in a liaHGF::kan mutant. The corresponding strains TMB096-TMB104 were inoculated in LB-medium to mid-log phase $\left(\mathrm{OD}_{600} \sim 0.4\right)$ and the cells from $2 \mathrm{ml}$ were harvested and used for $\beta$ galactosidase assay as described above (without the addition of bacitracin). The resulting $\beta$-galactosidase acitivities, expressed in Miller units (Miller, 1972), are shown. (D) Sequence of the promoter region upstream of $y h c Y$. The transcriptional start site is highlighted in bold, underlined, the promoter bold italics. The putative rhoindependent $y h x B$-terminator is indicated by arrows and highlighted in bold/underlined, coding regions are given in bold, with the start- und stop-codon underlined. The palindrome of the putative LiaR binding site is underlined and boxed. The 5'-end of the promoter fragments used for the promoter deletion analysis are marked. The minimal LiaR-dependent promoter fragment is shaded in grey. Note that the labeling of the 5'-end of the fragments for the promoter deletion analysis in this figure (Fig. 2.7C and 2.7D) is given relative to the transcription start (" +1 ") for reasons of clarity. Thereby, this nomenclature differs from the labeling of the fragments for cloning (Table 2.1 and 2.2), which are normalized relative to the "A" of the start codon of $y h c Y$. 
analysis verified that the $y h c Y Z-y h d A$ genes constitute an operon of $2.2 \mathrm{~kb}$ size (data not shown).

To further characterize the LiaR-dependent activity of $\mathrm{P}_{y h c}$ and the role of the putative LiaRbinding site, a promoter deletion analysis was performed. Promoter fragments of decreasing length were cloned into pAC6 and verified by DNA sequencing. Integration of the resulting plasmids generated strains TMB096 through TMB104 (smallest to largest $\mathrm{P}_{y h c Y}$ fragment, respectively, see Table 2.1 and Fig. 2.7C for details). The results demonstrate that a complete LiaR-box adjacent to the promoter region is necessary and sufficient for LiaR-dependent promoter activity (Fig. 2.7C and 2.7D).

Site-directed mutagenesis of the putative LiaR-binding site in $\mathbf{P}_{\text {liaI }}$. To experimentally test the importance of individual residues within the conserved LiaR-binding motif, we generated a series of LiaR-box mutants by site directed mutagenesis (Table 2.4). Exchanging the five most highly conserved nucleotides of the LiaR-box by an "A" led to a complete loss of promoter activity in strain TMB113. The same effect was observed with mutations affecting only the promoter-proximal half-side (TMB114 and TMB133). Interestingly, a mutant with the three highly conserved residues in the 5'-side of the palindrome replaced by " $A$ " (strain

Table 2.4. Mutagenesis of the putative LiaR-binding site upstream of $\mathbf{P}_{\text {lial }}$

\begin{tabular}{|c|c|c|c|}
\hline \multirow{2}{*}{$\begin{array}{l}\text { Strain } \\
\text { wildtype }\end{array}$} & \multirow{2}{*}{$\begin{array}{l}\text { Sequence of putative LiaR-binding } \text { site }^{2} \\
\text { CGGTGCGAGATACGAC CCGGTCTTATATAAAAATCAATCT }\end{array}$} & \multicolumn{2}{|c|}{$\begin{array}{l}\beta \text {-Gal }[\mathrm{MU}]^{3} \\
-\mathrm{Bac}+\end{array}$} \\
\hline & & 0,9 & 196 \\
\hline TMB113 & TTATATAAAAATCAATCT & $\mathbf{0 , 8}$ & 0,6 \\
\hline TMB115 & CGGTGCGAGAAACAAA & 0,5 & 4,5 \\
\hline TMB133 & CGGCGGGAGATACGACTCCGAAATTATATAAAAATCAATCT & 0,6 & 0,8 \\
\hline TMB114 & CGGTGCGAGATACGACFCCGGTCTTCTATACAAATCAATCT & 0,2 & 0,6 \\
\hline TMB111 & CGGTGCGAGATACGACFC & $\mathbf{0 , 3}$ & 13 \\
\hline TMB112 & CGGTGCGAG & 0,7 & 17 \\
\hline
\end{tabular}

\footnotetext{
${ }^{1}$ All strains harbor the minimal LiaR-dependent promoter fragment, transcriptionally fused to lacZ, integrated at the amyE locus (see Table 2.1 for details).

${ }^{2}$ The palindrome is highlighted by a black frame and the 7-4-7 motif is indicated. Conserved nucleotides are given in bold letters. Minimal LiaR-responsive promoter fragments were mutated at the nucleotides indicated by black boxes during PCR-amplification, cloned into pAC6 to generate mutated $\mathrm{P}_{\text {lial }}$ lac $Z$ fusions, sequence verified and subsequently transformed into W168 as described in the material and methods and results sections. ${ }^{3} \mathrm{P}_{\text {lial }}$-activity of the resulting mutants was determined by $\beta$-galactosidase assay with and without addition of bacitracin (Bac; final concentration $50 \mu \mathrm{g} / \mathrm{ml}$ ) as described in the legend to Fig. 2.1. MU = Miller units. TMB016 ("wildtype") is given as a reference.
} 
TMB115) retained a very low level of bacitracin-inducible activity. All three LiaR-boxes of B. subtilis harbor an A-stretch directly 3' of the inverted repeat, with two residues also being conserved in the putative binding sites of LiaR-homologs (Fig. 2.5). Therefore, we constructed two additional mutants (TMB111 and 112) each harboring a different set of three $A \rightarrow T$ exchanges. These mutants each showed a signficantly decreased, albeit less severely affected $\mathrm{P}_{\text {lial }}$-activity in the presence of bacitracin. These results support a functional role of this A-rich region, perhaps serving as a DNA-binding site (UP element) that interacts with the $\alpha$-C-terminal domain of RNA-polymerase (Gaal et al., 1996).

\section{Discussion}

A complex regulatory network, consisting of TCS and alternative $\sigma$-factors, orchestrates the cell envelope stress response in B. subtilis (Mascher et al., 2003; Pietiäinen et al., 2005). One such TCS, LiaRS, mediates the strong induction of its corresponding liaIHGFSR locus in the presence of antibiotics that interfere with the lipid II cycle, such as bacitracin, vancomycin, nisin, and ramoplanin (Mascher et al., 2004). The LiaRS TCS also responds strongly to the presence of cationic antimicrobial peptides known to affect the cell envelope (Pietiäinen et al., 2005) and is weakly induced in response to secretion stress, alkaline shock and the presence of detergents, organic solvents, ethanol and some surfactants (Hyyryläinen et al., 2005; Mascher et al., 2004; Petersohn et al., 2001; Wiegert et al., 2001). Here, we investigated the role of individual genes of the lia locus in LiaRS-mediated cell envelope stress response. We found that $\mathrm{LiaF}$ and $\mathrm{LiaH}$ act as a strong and weak inhibitor of this signal transduction process, respectively (Fig. 2.1 and Fig. 2.3). Applying comparative genomics, we identified a putative LiaR-binding site that was verified by mutational analysis (Fig. 2.5, Table 2.4). This LiaR-box was also found in the promoter region of a second LiaR-target locus, the $y h c Y Z$ $y h d A$ operon, the expression of which we studied in detail (Fig. 2.7).

\section{LiaRS-mediated signal transduction is conserved in Firmicutes bacteria. Using} comparative genomics, we recognized LiaF-LiaRS as a cell envelope stress response system conserved in the phylum Firmicutes (Fig. 2.4). We extended our comparative genomics studies to identify putative LiaR-binding sites and corresponding target genes in other Firmicutes bacteria (Table 2.5). LiaR-boxes were primarily identified in organisms of the order Bacillales, harboring both liaFSR and liaIH homologs. Overall, the number of putative 


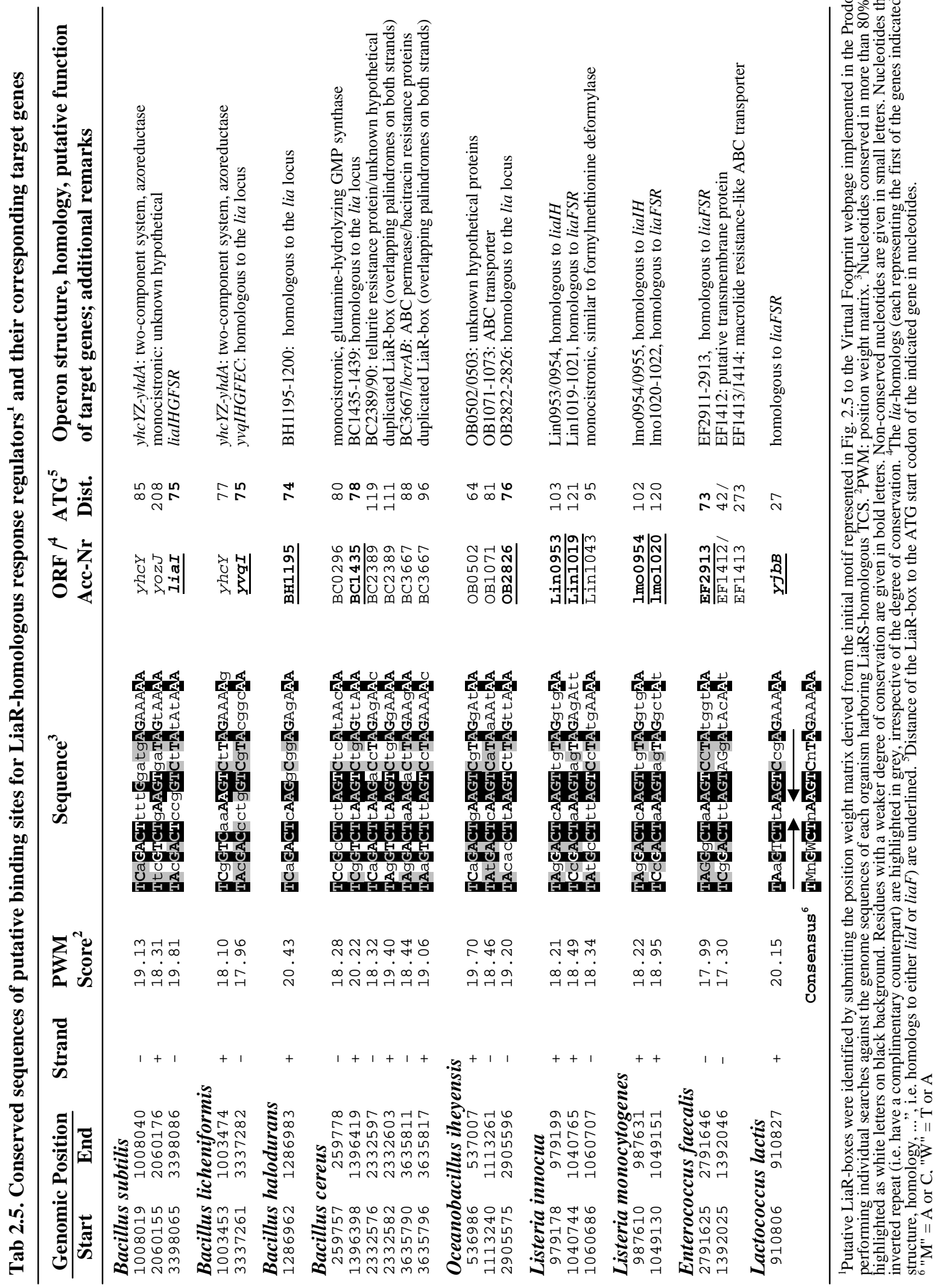


LiaR-binding sites per genome was low (one to four). In B. licheniformis, the closest sequenced relative to $B$. subtilis, the two putative LiaR-binding sites are also located upstream of homologous loci, i.e. $y v q I$ (liaI) and $y h c Y$. For the two Listeria species, the homologs of liaIH (first gene lin0953/Lmo0954) and liaFSR (lin1019/Lmo1020) are organized in two independent transcriptional units, as noted above (Fig. 2.4). Interestingly, a putative LiaRbinding site was identified in the promoter regions of both loci. A LiaR-box was also identified upstream of the liaF-homologs in Enterococcus faecalis and Lactococcus lactis (Table 2.5, and triangles in Fig. 2.4). Remarkably, three of the candidate target loci encode ABC transporters (including a putative bacitracin efflux pump), supporting the role of LiaRShomologs in mediating a cell envelope stress response (Table 2.5).

The sequence alignment of putative LiaR-boxes identified a core motif of 16 nucleotides, followed by an "A"-rich 3' extension that could function as an UP element (Gaal et al., 1996). The core motif consists of an inverted repeat of seven nucleotides, with a spacing of two or four nucleotides. LiaR belongs to the NarL/FixJ family of response regulators (Pao et al., 1994). The recognition of an inverted repeat is well established for this family of transcriptional regulators (Crater and Moran, 2001; Dahl et al., 1997; Egland and Greenberg, 2000) as well as for other response regulators such as L. monocytogenes VirR and Bradyrhizobium japonicum RegR (Emmerich et al., 2000; Mandin et al., 2005).

The conservation of the LiaF-LiaRS signal transduction system and several of its target operons in the Firmicutes (with the noteworthy exception of the genus Clostridium) (Fig. 2.4) suggests that this is a conserved cell envelope stress system. Preliminary transcriptome analyses revealed that the lia-homologous yvqIHGFEC locus in B. licheniformis is also strongly induced by bacitracin (Wecke and Mascher, unpublished observation). Other LiaRS homologs, such as Staphylococcus aureus VraSR, Listeria monocytogenes Lmo1021/1022, and S. pneumoniae HK03/RR03 also respond to cell envelope stress elicited by cell wall antibiotics and membrane perturbations (Gravesen et al., 2004; Haas et al., 2005; Kuroda et al., 2003). In the case of S. aureus, the VraSR-system controls a large regulon of about 50 genes (Kuroda et al., 2003). It seems reasonable to postulate that all liaFSR homologs depicted in Fig. 2.4 encode cell envelope stress sensing three-component systems. Although liaFSR-like operons are present in the streptococci and staphylococci, we were unable to identify LiaR-boxes upstream of these loci. We speculate that the corresponding LiaR-like proteins have a distinct DNA-binding selectivity, a hypothesis supported by the divergence of their DNA-binding domains (data not shown). 
Taken together, the data seem to indicate the existence of two sub-groups within LiaRS-like cell envelope stress-sensing TCS. The genera Bacillus and Listeria harbor the complete lia locus (organized as two independent, but LiaR-dependent transcriptional units for the latter). In B. subtilis, one prominent effect of activation is a strong overexpression of $\mathrm{LiaH}$ and, due to the operon organization, presumably also of the small membrane protein LiaI. In general, these systems have recognizable LiaR-binding sites (exception: $B$. anthracis and $B$. stearothermophilus) and seem to control only a small number of target genes (Table 2.5). Members of the second group (VraSR-like TCS) lack a recognizable LiaR-box and homologs of liaIH, but seem to control a larger regulon.

LiaF is a strong inhibitor of LiaRS-mediated signal transduction. We identified LiaF as an essential part of the LiaRS signaling system that maintains the system in an inactive state (Fig. 2.1, 2.3, and 2.7A). It is presently unclear whether LiaF senses cell envelope stress directly or indirectly through LiaS. Most characterized proteins inhibiting TCS-mediated signal transduction - such as Sda and KipI in the sporulation phosphorelay of B. subtilis, and FixT in FixLJ-mediated nitrogen fixation in Sinorhizobium meliloti - interfere with histidine kinase autophosphorylation, thereby suppressing activation of the cognate response regulators (Burkholder et al., 2001; Garnerone et al., 1999; Wang et al., 1997). Recently, a TCSinhibitor protein was described that shares many features with LiaF: Deletion of $y y c H$ encoding a transmembrane protein - results in uncoupled activity of YycFG (Szurmant et al., 2005), an essential TCS in B. subtilis and other Gram-positive bacteria (Clausen et al., 2003; Fabret and Hoch, 1998). The three corresponding genes are co-transcribed and conserved by genomic context. It has been postulated that $\mathrm{YycH}$ affects the sensor domain of its corresponding histidine kinase, YycG (Szurmant et al., 2005). A similar role could be envisioned for LiaF.

LiaH, a phage-shock protein A (PspA) homolog, acts a negative modulator of LiaRSmediated signal transduction. We also presented evidence that LiaH acts as a weak - and presumably indirect - negative modulator for LiaR-dependent gene expression. The liaIH genes are expressed at a much higher level relative to the downstream genes liaGFSR, due to termination of transcription at a stem-loop structure downstream of liaH (Mascher et al., 2004). The strong induction of liaIH is also apparent at the protein level: LiaH was described as a marker protein for bacitracin treatment in a proteomic study (Bandow et al., 2003). Furthermore, LiaH is a highly abundant protein visible in one-dimensional SDS-PAGE of cell lysates from cultures of the liaF-mutant, even in the absence of bacitracin (data not shown). 
LiaH belongs to the family of phage-shock proteins. So far, only two members of this protein family have been investigated in detail, PspA of Yersina enterocolitica and E. coli. The latter is induced by filamentous phage infection (Brissette et al., 1990), hence the name phageshock protein. It is also induced by various other stress conditions, such as heat shock, osmotic shock, exposure to organic solvents and proton ionophores and long incubation under alkaline conditions (Brissette et al., 1990; Kobayashi et al., 1998; Weiner and Model, 1994). Furthermore, misincorporation of secretin pore proteins also induces the $p s p$ locus in both $Y$. enterocolitica and E. coli, as does blocking of the Sec pathway (Darwin and Miller, 2001; Hardie et al., 1996; Kleerebezem and Tommassen, 1993; Kleerebezem et al., 1996). In comparison, LiaH expression is induced by cell envelope stress generated by lipid IIinteracting antibiotics such as bacitracin and ramoplanin, but also by cationic antimicrobial peptides, alkaline shock, exposure to organic solvents, detergents, ethanol and secretion stress (Hyyryläinen et al., 2005; Mascher et al., 2003; Petersohn et al., 2001; Pietiäinen et al., 2005; Wiegert et al., 2001). While the mechanism of their transcriptional regulation differs, the range of inducing conditions for B. subtilis LiaH and E. coli PspA is remarkably similar. PspA exhibits a dual function that is linked to two different cellular locations (Brissette et al., 1990; Kleerebezem and Tommassen, 1993): in unstressed cells, it is a cytosolic protein that acts as a negative regulator by inhibiting the transcriptional activator PspF (Adams et al., 2003; Bordes et al., 2003; Dworkin et al., 2000). Under conditions of cell envelope stress (leaky outer membrane), it is peripherally bound to the inner surface of the cytoplasmic membrane, contributing to the maintenance of the proton motive force and overall membrane integrity (Kleerebezem et al., 1996). This membrane anchoring is mediated by protein-protein interaction with two transmembrane proteins, PspB and PspC (Adams et al., 2003; Bordes et al., 2003; Dworkin et al., 2000). Based on our findings, it is tempting to postulate a similar dual role for LiaH. It functions as a weak negative transcriptional regulator without having a DNA-binding domain (Fig. 2.1 and Fig. 2.7A). Its cotranscription with liaI, coding for a putative membrane protein, suggests that LiaI might serve as a membrane anchor for LiaH, thereby facilitating a second (so far unknown) activity, linked to the inner surface of the cytoplasmic membrane.

LiaFRS-mediated gene regulation - a working model. Under normal growth conditions, the last four genes of the lia locus, liaGFSR, are expressed from a recently identified, weak constitutive promoter directly upstream of liaG (Fig. 2.8A, and data not shown; note the 

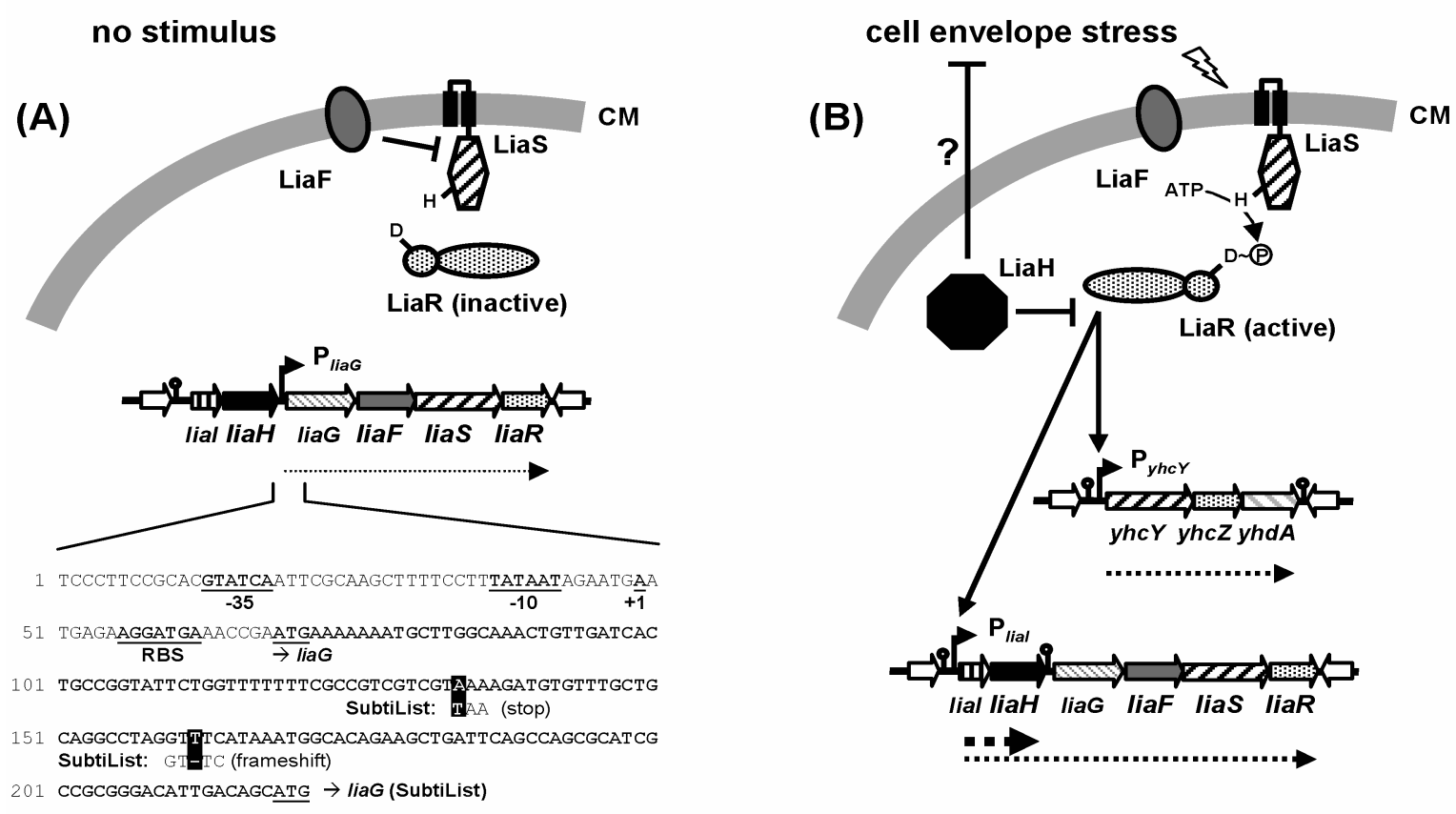

Figure 2.8. Model of LiaRS-dependent gene expression in B. subtilis. Transcripts are indicated by dotted lines. Activation is indicated by solid arrows, inhibitions by T-shaped lines. (A) $\mathrm{P}_{\text {liaG }}$-dependent expression of liaGFSR in the absence of cell envelope stress. LiaF inhibits the LiaRS TCS. The sequence of the corresponding promoter region is given below. Re-sequencing revealed two mistakes in the original genome sequence, which result in the generation of a stop codon and a frame-shift within the 5'-end of liaG, respectively. Therefore, the corresponding $\mathrm{LiaG}$ protein is extended by another 50 amino acids at its N-terminus. The added sequence harbors a signal peptide and a potential transmembrane helix, as identified using the SMART database (Schultz et al., 1998). This indicates that LiaG is a putative membrane-anchored protein rather than of cytoplasmic localization, as was originally annotated. (B) LiaRS-dependent gene expression in the presence of cell envelope stress. LiaF repression is released and activated LiaR binds to its target promoters, including its own operon (positive feedback loop). The strong overexpression of LiaH presumably counteracts cell envelope stress. Additionally LiaH functions as an inhibitor of LiaR-dependent gene expression (negative feedback loop). See text for further details.

corrected sequence for liaG; see Materials and Methods). $\mathrm{P}_{l i a G}$-dependent expression and the inhibitory activity of LiaF ensure that the system is present but switched off (Fig. 2.8A). In the presence of cell envelope stress, LiaS is released from LiaF-dependent inhibition and phosphorylated LiaR binds upstream of $\mathrm{P}_{l i a I}$ and $\mathrm{P}_{y h c Y}$ (Fig. 2.8B). $\mathrm{P}_{\text {lial }}$-induction gives rise to two transcripts, resulting most notably in overexpression of LiaH. Due to significant readthrough transcription, this induction defines a positive autoregulatory feedback-loop (stress-induced expression of liaGFSR) that allows the system to rapidly respond to envelope stress. The importance of this positive feedback loop is underscored by the identification of candidate LiaR-boxes in the promoter regions of those liaFSR homologs that do not receive readthrough transcription from upstream liaIH expression (Fig. 2.4), i.e. in Listeria species. The physiological role of LiaH and the other LiaR targets is not yet clear. Based on its homology to PspA, we speculate that LiaH might be involved in counteracting envelope stress, possibly by securing membrane integrity. Additionally, it plays a role in a negative feedback loop, thereby counteracting continued LiaR activity. This allows the system to 
establish a level of LiaH appropriate for the stress condition (Fig. 2.8B). Two possible mechanisms for this LiaH-dependent feedback loop can be envisioned, both based on the analogy to E. coli PspA: first, LiaH could regulate LiaR-activity through direct proteinprotein interaction as suggested above. Second, LiaH could help to restore envelope integrity, thereby removing the stress signal that activated LiaRS in the first place. In the absence of envelope stress, LiaF would then again be able to inhibit LiaRS, thereby efficiently switching the system off. It is more and more recognized that such combined positive and negative feedback loops in a regulatory pathway play an important role for adaptive responses of a bacterial population to its environment (i.e. competence and sporulation in B. subtilis), allowing a fast and differentiated response (Smits et al., 2006).

\section{Acknowledgements}

We thank Sierd Bron for the gift of strains BFS1714 and BFS1715 and Tarek Msadek for gift of plasmid pXT. Furthermore, we would like to gratefully acknowledge the contributions of a number of students, who were involved in various aspects of the results described herein during their lab rotation projects (in order of appearance): Sara L. Zimmer (Cornell University) for primer extension of $\mathrm{P}_{y h c Y}$, Terry-Ann Smith (NYU) and Tina Wecke for contributing to the initial mutational analysis of the lia locus, Falk Kalamorz for SDS-PAGE analysis of LiaH overexpression, Diana Hoyer for taking part in the identification of $\mathrm{P}_{\text {liaG }}$ and Bastian Dörrbecker for the mutagenesis of the LiaR-binding site of $\mathrm{P}_{\text {lial }}$. This work was supported by grant MA 3269 from the Deutsche Forschungsgemeinschaft and grants from the Fonds der Chemischen Industrie (to TM) and by grant GM-47446 from the National Institutes of Health (to JDH). 


\section{Chapter 3:}

\section{LiaRS-Dependent Gene Expression is Embedded in Transition State Regulation in Bacillus subtilis}

The work described in this chapter was published in:

Jordan, S., Rietkötter, E., Strauch, M. A., Kalamorz, F., Butcher, B. G., Helmann, J. D., and Mascher, T. 2007. LiaRS-dependent gene expression is embedded in transition state regulation in Bacillus subtilis. Microbiology 153: 2530-2540

Author contributions:

Sina Jordan performed the experiments and draw the figures. Eva Rietkötter contributed to the construction of mutants and performed $\beta$-galactosidase measurements in a practical training. Mark A. Strauch performed the DNase I footprint. Falk Kalamorz provided the LiaH antibodies. Bronwyn G. Butcher contributed to the initial results in the laboratory of John D. Helmann. 


\section{Summary}

Maintaining envelope integrity is crucial for the survival of any bacterial cell, especially those living in a complex and ever-changing habitat such as the soil ecosystem. The LiaRS TCS is part of the regulatory network orchestrating cell envelope stress response in Bacillus subtilis. It responds to perturbations of the cell envelope, especially the presence of antibiotics that interfere with the lipid II cycle, such as bacitracin or vancomycin. LiaRS-dependent regulation is strictly repressed by the membrane protein LiaF in the absence of inducing conditions. Here, we show that the LiaR-dependent liaI promoter is induced at the onset of stationary phase without addition of exogenous stresses. Its activity is embedded in the complex regulatory cascade governing adaptation at the onset of stationary phase: The liaI promoter is directly repressed by the transition state regulator $\mathrm{AbrB}$ and responds indirectly to the activity of Spo0A, the master regulator of sporulation. The activity of the liaI promoter is therefore tightly regulated by at least five regulators to ensure an appropriate level of liaIH expression.

\section{Introduction}

The envelope is a crucial structure of the bacterial cell and the target of many antibiotics (Silver, 2003, 2006; Walsh, 2003). Its integrity is closely monitored to detect and counteract threats before their action can lead to irreversible damages. The LiaRS two-component system (TCS) is part of the complex regulatory network that orchestrates cell envelope stress response in B. subtilis (Mascher et al., 2003). It strongly responds to the external presence of cell wall antibiotics that interfere with the lipid II cycle, such as bacitracin, ramoplanin, vancomycin, or cationic antimicrobial peptides (Mascher et al., 2004; Pietiäinen et al., 2005). It is also induced by alkaline shock, detergents, ethanol, phenol, organic solvents, and secretion stress, albeit to a lesser extent (Hyyryläinen et al., 2005; Mascher et al., 2004; Petersohn et al., 2001; Pietiäinen et al., 2005; Tam le et al., 2006; Wiegert et al., 2001).

The LiaRS TCS is functionally and genetically linked to a third protein, LiaF, which acts as a strong inhibitor of LiaR-dependent gene expression (Jordan et al., 2006). The LiaRS-LiaF three-component system is conserved by sequence and genomic context in Gram-positive bacteria with a low G+C content (Firmicutes) (Jordan et al., 2006; Mascher, 2006b), and LiaRS-homologous TCS are also involved in responding to cell envelope stress in Bacillus 
licheniformis, Streptococcus pneumoniae, and Staphylococcus aureus (Haas et al., 2005; Kuroda et al., 2003; Wecke et al., 2006). It is interesting to note that membrane anchored inhibitory proteins, working together with a classical TCS, have also been described for the cell wall related, essential TCS YycFG: the YycH and YycI proteins both inhibit the YycG kinase (Szurmant et al., 2007a).

In B. subtilis, only two promoters are known to be regulated by the LiaRS TCS: the liaI promoter $\left(\mathrm{P}_{\text {lial }}\right)$ and the $y h c Y$ promoter (Jordan et al., 2006), with $\mathrm{P}_{\text {lial }}$ being the primary

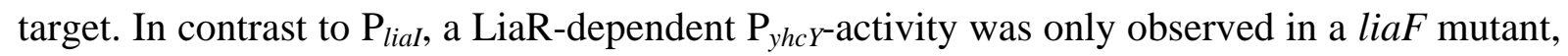
i.e. in the absence of the LiaRS-inhibitor protein (Jordan et al., 2006; Mascher et al., 2004). $\mathrm{P}_{\text {lial }}$ is tightly regulated: in the absence of a stimulus, it is virtually switched off, while addition of bacitracin results in an about 200-fold induction (Mascher et al., 2003; Mascher et al., 2004).

The lia locus consists of six genes, liaIH-liaGFSR. A basal expression level of the last four genes, liaGFSR, encoding the three-component system (liaFSR) and a putative membraneanchored hypothetical protein $(l i a G)$, is ensured by a weak constitutive promoter upstream of liaG. In contrast, expression of the liaIH operon from $\mathrm{P}_{\text {lial }}$ is completely LiaR-dependent (Jordan et al., 2006). LiaI is a small hydrophobic protein of unknown function with two putative transmembrane helices. $\mathrm{LiaH}$ is a member of the phage-shock protein family (see below). While the strong induction of liaIH (and to a lesser degree also liaGFSR) by cell envelope stress is well documented (see above), mutational analyses of the lia locus so far failed to identify strong phenotypes associated with them. Deletion of lia genes did not alter the sensitivity of the corresponding mutants to the known inducers of the Lia system. Moreover, none of the complex differentiation processes of B. subtilis (i.e. sporulation, competence for genetic transformation, motility, pellicle and fruiting body formation) was affected in lia mutants. So far, the only phenotype that could be linked to the Lia system is delayed spore germination in a liaH mutant (Hoyer \& Mascher, unpublished). Moreover, LiaH seems to negatively affect the expression of the $y h c Y Z$ operon by a so far unknown mechanism. It is weakly inducible by bacitracin only in a liaH mutant, but not in the wild type (Mascher et al., 2003).

While the physiological role of LiaI and LiaH remains obscure, we noted some similarities between LiaH and phage-shock protein A (PspA) of E. coli. The latter is induced by various stress conditions such as filamentous phage infection (hence the name), heat shock, osmotic shock, exposure to organic solvents and proton ionophores as well as long incubation under alkaline conditions (Brissette et al., 1990; Kobayashi et al., 1998; Weiner and Model, 1994). 
This inducer spectrum shows some overlap with the known inducers of lialH expression, which include organic solvents and alkaline shock (Mascher et al., 2004; Wiegert et al., 2001). PspA exhibits a dual function that is linked to two different cellular locations (Brissette et al., 1990; Kleerebezem and Tommassen, 1993): Peripherally bound to the inner surface of the cytoplasmic membrane (through protein-protein interactions), PspA is somehow involved in the maintenance of cell membrane integrity (Darwin, 2005; Kleerebezem et al., 1996). As a free cytosolic protein it inhibits the AAA+ enhancer protein PspF, also through proteinprotein interactions (Adams et al., 2003; Bordes et al., 2003; Dworkin et al., 2000). Based on the strong induction of liaH by cell envelope stress and its co-transcription with liaI, coding for a small putative membrane protein, we speculate that LiaI serves as a membrane anchor for LiaH, thereby facilitating an activity that might somehow be linked to envelope integrity. Here, we investigated the intrinsic activity and regulation of $\mathrm{P}_{\text {lial }}$ in the absence of exogenous stimuli. We show that $\mathrm{P}_{\text {lial }}$ is induced at the onset of stationary phase. This time point in the $B$. subtilis life cycle is characterized by the initiation of a complex regulatory cascade that allows Bacillus to adapt to worsening living conditions, which can ultimately lead to the formation of dormant endospores (Errington, 2003; Msadek, 1999; Phillips and Strauch, 2002). We demonstrate that $\mathrm{P}_{\text {lial }}$ is directly repressed by binding of the transition state regulator $\mathrm{AbrB}$ within the promoter sequence, thereby acting as a roadblock to prevent premature $\mathrm{P}_{\text {lial }}$ activity during logarithmic growth. AbrB repression is released during the transition state by Spo0A the master regulator of sporulation and $\mathrm{P}_{\text {lial }}$ is induced by an unidentified endogenous stimulus, resulting in the expression of the liaIH operon. While AbrB-binding is sufficient to inhibit the endogenous growth-dependent induction of $\mathrm{P}_{\text {lial }}$, it can be bypassed completely by exogenous induction with cell wall antibiotics.

\section{Methods}

Bacterial strains and growth conditions. B. subtilis was routinely grown in LB medium at $37^{\circ} \mathrm{C}$ with aeration. All strains used in this study are derivatives of the wild type strain W168 and are listed in Table 3.1. Kanamycin $(10 \mu \mathrm{g} / \mathrm{ml})$, chloramphenicol $(5 \mu \mathrm{g} / \mathrm{ml})$, spectinomycin $(100 \mu \mathrm{g} / \mathrm{ml})$, tetracyclin $(10 \mu \mathrm{g} / \mathrm{ml})$, and erythromycin $(1 \mu \mathrm{g} / \mathrm{ml})$ plus lincomycin $(25 \mu \mathrm{g} / \mathrm{ml})$ for macrolide-lincosamide-streptogram ("MLS") resistance, were used for the selection of the B. subtilis mutants used in this study. Transformation was carried out as described (Harwood and Cutting, 1990). 
Allelic replacement mutagenesis using Long Flanking Homology (LFH-) PCR. This technique is derived from the published procedure (Wach, 1996) and was performed as described previously (Mascher et al., 2003). In brief: resistance cassettes were amplified from a suitable vector as template (Guerout-Fleury et al., 1995; Youngman, 1990). Two primer pairs were designed to amplify $\sim 1000$ bp DNA-fragments flanking the region to be deleted at its 5'- and 3'-end. The resulting fragments are here called 'up' and 'do' fragment. The 3'-end of the up-fragment as well as the 5'-end of the do-fragment extended into the gene(s) to be deleted in a way that all expression signals of genes up- and downstream of the targeted genes remained intact. Extensions of $\sim 25$ nucleotides were added to the 5'-end of the 'up-reverse' and the 'do-forward' primers that were complementary (opposite strand and inverted sequence) to the 5'- and 3'-end of the amplified resistance cassette. All obtained fragments were purified using the PCR-purification kit from Qiagen. 100-150 ng of the up- and dofragments and 250-300 ng of the resistance cassette were used together with the specific upforward and do-reverse primers at standard concentrations in a second PCR-reaction. In this reaction the three fragments were joined by the 25 nucleotide overlapping complementary ends and simultaneously amplified by normal primer annealing. The PCR-products were directly used to transform $B$. subtilis. Transformants were screened by colony-PCR, using the up-forward primer with a reverse check-primer annealing inside the resistance cassette (Table 3.2). The integrity of the regions flanking the integrated resistance cassettes was verified by sequencing PCR products of $\sim 1000 \mathrm{bp}$ amplified from chromosomal DNA of the resulting mutants. Sequencing was performed in house by the GenoMIK center. All PCR-reactions were done in a total volume of $50 \mu \mathrm{l}(10 \mu \mathrm{l}$ for colony PCR) using the HotStar DNAPolymerase Mastermix (Qiagen) or TripleMaster Polymerase Mix (Eppendorf) according to the manufacturer's procedure. The constructed strains are listed in Table 3.1. The primers used in this study are listed in Table 3.2.

Construction of a clean liaS deletion mutant. To ensure "normal" (i.e. wild type) expression levels of liaR, we constructed a clean deletion of liaS using the vector pMAD (Arnaud et al., 2004). The genomic regions $\sim 1 \mathrm{~kb}$ upstream and downstream of liaS were amplified using primers listed in Table 3.2 (fragments: liaS(clean) up, and liaS(clean) down), thereby introducing a 26 bp extension to the $3^{\prime}$-end of the up-fragment, which is complementary to the $5^{\prime}$-end of the down-fragment. The two fragments were fused in a second joining PCR reaction, and the resulting fragment cloned into pMAD via BamHI and EcoRI, generating pMM101. Generation of the clean deletion basically follows the established procedure (Arnaud et al., 2004). In brief: B. subtilis W168 was transformed with 
pMM101 and incubated at $30^{\circ} \mathrm{C}$ with MLS selection on LB agar plates supplemented with XGal. Blue colonies were picked and incubated for $6-8 \mathrm{~h}$ at $42^{\circ} \mathrm{C}$ in $\mathrm{LB}$ medium with MLS selection, resulting in the integration of pMM101 into the chromosome. Again, blue colonies were picked from LB (X-Gal) plates and incubated for $6 \mathrm{~h}$ in LB medium without selection. Subsequently, the liquid culture was shifted to $42^{\circ} \mathrm{C}$ for $3 \mathrm{~h}$, and the cells were then plated on LB (X-Gal) plates, this time without selective pressure. White colonies that had lost the plasmid were picked and checked for MLS sensitivity. Those harbouring a clean deletion of liaS ( 50\% of the white clones) were identified by PCR.

Table 3.1. Strains used in this study

\begin{tabular}{|c|c|c|}
\hline strains & relevant genotype $^{1}$ & source, reference, construction ${ }^{2}$ \\
\hline W168 & wild type strain, $\operatorname{trp} C 2$ & lab stock \\
\hline BFS2470 & liaI::pMUTIN & (Mascher et al., 2003) \\
\hline НВ0933 & 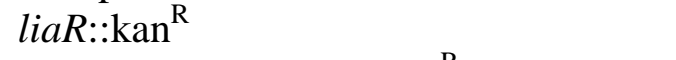 & (Mascher et al., 2003) \\
\hline TMB011 & liaI::pMUTIN, liaR::kan ${ }^{\mathrm{R}}$ & chrom. DNA (HB0933) $\rightarrow$ BFS2470 \\
\hline TMB079 & $\sin R:: \operatorname{spec}^{\mathrm{R}}$ & LFH-PCR $\rightarrow$ W168 \\
\hline TMB080 & $\operatorname{aprE::} \operatorname{kan}^{\mathrm{R}}$ & LFH-PCR $\rightarrow$ W168 \\
\hline TMB081 & scoC::tet ${ }^{\mathrm{R}}$ & LFH-PCR $\rightarrow$ W168 \\
\hline TMB082 & $a b r B:: \mathrm{kan}^{\mathrm{R}}$ & LFH-PCR $\rightarrow$ W168 \\
\hline TMB083 & salA ::tet ${ }^{\mathrm{R}}$ & LFH-PCR $\rightarrow$ W168 \\
\hline TMB084 & lial::pMUTIN, $\sin R:: \operatorname{spec}^{\mathrm{R}}$ & chrom. DNA (TMB079) $\rightarrow$ BFS2470 \\
\hline TMB085 & 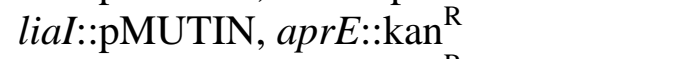 & chrom. DNA (TMB080) $\rightarrow$ BFS2470 \\
\hline TMB086 & lial::pMUTIN, scoC:: $\operatorname{kan}^{\mathrm{R}}$ & chrom. DNA (TMB081) $\rightarrow$ BFS2470 \\
\hline TMB087 & liaI::pMUTIN $a b r B:: \mathrm{kan}^{\mathrm{R}}$ & chrom. DNA (TMB082) $\rightarrow$ BFS2470 \\
\hline TMB088 & liaI::pMUTIN salA::tet ${ }^{\mathrm{R}}$ & chrom. DNA (TMB083) $\rightarrow$ BFS2470 \\
\hline TMB117 & liaI::pMUTIN $\operatorname{deg} U:: \mathrm{kan}^{\mathrm{R}}$ & chrom. DNA (TMB124) $\rightarrow$ BFS2470 \\
\hline TMB118 & liaI::pMUTIN spoOA::tet ${ }^{\mathrm{R}}$ & chrom. DNA (TMB205) $\rightarrow$ BFS2470 \\
\hline TMB124 & $\operatorname{deg} U:: \mathrm{kan}^{\mathrm{R}}$ & LFH-PCR $\rightarrow$ W168 \\
\hline TMB205 & spo0A::tet ${ }^{\mathrm{R}}$ & LFH-PCR $\rightarrow$ W168 \\
\hline TMB209 & 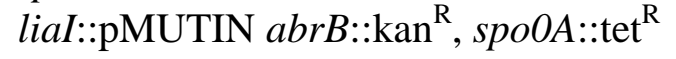 & chrom. DNA (TMB082) $\rightarrow$ TMB118 \\
\hline TMB213 & $\Delta l i a S$ & pMAD-based clean deletion \\
\hline TMB215 & $\Delta$ lias, liaI::pMUTIN & chrom. DNA (BFS2470) $\rightarrow$ TMB213 \\
\hline TMB330 & $\Delta l i a S$, liaI::pMUTIN, abrB::kan ${ }^{\mathrm{R}}$ & chrom. DNA (TMB082) $\rightarrow$ TMB215 \\
\hline
\end{tabular}

${ }^{1} \mathrm{kan}^{\mathrm{R}}$, kanamycin; $\operatorname{spec}^{\mathrm{R}}$, spectinomycin; tet ${ }^{\mathrm{R}}$, tetracycline resistance cassette

${ }^{2}$ All deletion mutants were constructed by replacing the corresponding gene with a resistance cassette, applying the long-flanking homology PCR strategy (LFH-PCR) as described previously (Mascher et al., 2003; Wach, 1996). See Table 2 for sequences of the primers used for their construction. Subsequent strains were constructed transformation of a recipient strain, given after the arrow, with chromosomal (chrom.) DNA from the donor strain (in parenthesis).

Measurement of induction by $\beta$-galactosidase assay. For time course experiments of $\mathrm{P}_{\text {lial }}{ }^{-}$ induction, the respective reporter strains (listed in Table 9) were inoculated in LB medium from a fresh mid-logarithmic pre-culture to an $\mathrm{OD}_{600} \approx 0.1$ and incubated at $37^{\circ} \mathrm{C}$ with 
aeration. $2 \mathrm{ml}$ samples were taken every hour and the $\mathrm{OD}_{600}$ was monitored to follow the growth of the cultures. The pellets were resuspended in $1 \mathrm{ml}$ of working buffer $(60 \mathrm{mM}$ $\mathrm{Na}_{2} \mathrm{HPO}_{4}, 40 \mathrm{mM} \mathrm{NaH} \mathrm{PO}_{4}, 10 \mathrm{mM} \mathrm{KCl}, 1 \mathrm{mM} \mathrm{MgSO} 4,20 \mathrm{mM} \beta$-mercaptoethanol) and suitable dilutions were assayed for $\beta$-galactosidase activity as described with normalization to cell density (Miller, 1972). For induction experiments, the cells were inoculated from fresh overnight cultures and grown in LB-medium at $37^{\circ} \mathrm{C}$ with aeration until they reached an $\mathrm{OD}_{600} \approx 0.6$. The culture was split, adding bacitracin $(50 \mu \mathrm{g} / \mathrm{ml}$ final concentration $)$ to one half (induced sample) and leaving the other half untreated (uninduced control). After incubation for an additional $30 \mathrm{~min}$ at $37^{\circ} \mathrm{C}$ with aeration, $2 \mathrm{ml}$ of each culture were harvested and the cell pellets were frozen and kept at $-20^{\circ} \mathrm{C}$. The pellets were resuspended in $1 \mathrm{ml}$ of working buffer and assayed for $\beta$-galactosidase activity as described with normalization to cell density (Miller, 1972).

Western blot. Total cytoplasmic proteins were prepared from $15 \mathrm{ml}$ of culture per time point by using a french press. $20 \mu \mathrm{g}$ of proteins per lane were separated by SDS-PAGE, according to standard procedure (Sambrook and Russell, 2001). After electrophoresis the gels were equilibrated in transfer buffer (15.2 g Tris; $72.1 \mathrm{~g}$ glycine; $750 \mathrm{ml}$ methanol (100 \%) in a final volume of 51 with de-ionized water) for 30 seconds. A PVDF membrane was activated with methanol (100\%) and subsequently incubated in transfer buffer for five minutes. The proteins were blotted to this membrane using a Semi Dry Blot apparatus. After transfer $(1 \mathrm{~h}$ at 0.8 $\left.\mathrm{mA} / \mathrm{cm}^{2}\right)$ the membrane was incubated in blotto (1x TBS $(50 \mathrm{mM}$ Tris, $150 \mathrm{mM} \mathrm{NaCl}, \mathrm{pH}$ 7.6), $2.5 \%$ skim milk) overnight to prevent unspecific binding. The LiaH antibody (polyclonal rabbit antisera that were raised against purified His 10 -LiaH (Kalamorz \& Mascher, unpublished) at SEQLAB, Göttingen, Germany) was diluted 1:20,000 in blotto. After incubation for three hours, the membrane was washed three times for 30 minutes with blotto. The secondary antibody (anti rabbit IgG, coupled with alkaline phosphatase, Roche Diagnostics, Mannheim) was diluted 1:100,000 and the membrane was incubated for 30 minutes. After three more washing steps for 20 minutes, the membrane was washed with deionized water and incubated in buffer III ( $0.1 \mathrm{M}$ Tris; $0.1 \mathrm{M} \mathrm{NaCl} ; \mathrm{pH} 9.5)$ for five minutes to adjust the pH. $10 \mu \mathrm{l} \mathrm{CDP-Star} \mathrm{(chemiluminescence} \mathrm{substrate,} \mathrm{Roche} \mathrm{Diagnostics,} \mathrm{Mannheim)}$ in $1 \mathrm{ml}$ buffer III were used for LiaH detection. The signal was documented with ChemiSmart LumiImager (peqlab).

DNase I footprinting assays. AbrB and Abh purification and DNase I footprinting assays were performed essentially as described previously (Bobay et al., 2006; Strauch et al., 1989). The DNA target was a 287 bp fragment containing the liaI promoter region (positions -159 to 
+128 ), end-labeled with ${ }^{32} \mathrm{P}$ on the template strand. The binding reactions were performed at $20^{\circ} \mathrm{C}$ with $\mathrm{pH} 8$ for $\mathrm{AbrB}$, and $\mathrm{pH} 7$ for Abh (the optimal pH's for each protein's binding) (Bobay et al., 2006).

Table 3.2. Oligonucleotides used in this study

\begin{tabular}{|c|c|}
\hline fragment $^{1}$ & primer sequence $^{2}$ \\
\hline aprE-up & $\begin{array}{l}\text { fwd: GTTGACATTCGGCACACTCC, } \\
\text { rev: CCTATCACCTCAAATGGTTCGCTGGACATGTTGCTGAACGCCATCG }\end{array}$ \\
\hline aprE-down & $\begin{array}{l}\text { fwd: } \frac{\text { CGAGCGCCTACGAGGAATTTGTATCGAAACGCGCAAGTCCGTGATCG, }}{\text { rev: }} \text { CATTTCCACACAGACAACGG }\end{array}$ \\
\hline salA-up & $\begin{array}{l}\text { fwd: AAGATTGGTGGACAGCAGG, } \\
\text { rev: CCTATCACCTCAAATGGTTCGCTGGGTTCGCGCATTTCTCCG }\end{array}$ \\
\hline salA-down & $\begin{array}{l}\text { fwd: } \overline{\text { CGAGCGCCTACGAGGAATTTGTATCGTGACGAAAATCATCCAATCGG, }} \text { rev: TATCTCAAGCGCAAACCGATG }\end{array}$ \\
\hline abrB-up & $\begin{array}{l}\text { fwd: TATCAACGAGCTGAGTTTCCG, } \\
\text { rev: CCTATCACCTCAAATGGTTCGCTGCAACTTTACGTACAATACCAGTAG }\end{array}$ \\
\hline abrB-down & 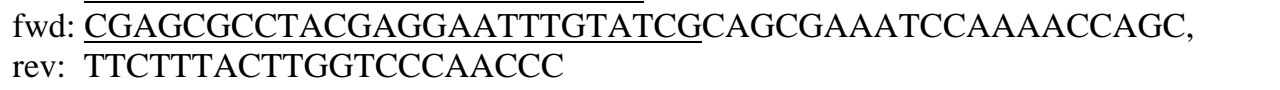 \\
\hline scoC-up & $\begin{array}{l}\text { fwd: AACCTCTTCCGCTTCCGG, } \\
\text { rev: CCTATCACCTCAAATGGTTCGCTGGAGCCTTGCTAAGCTGAGCC }\end{array}$ \\
\hline scoC-down & $\begin{array}{l}\text { fwd: } \overline{\text { CGAGCGCCTACGAGGAATTTGTATCGGATGAACCGGCTGAAGAGC, }} \text { rev: } \\
\text { ACGTTTCCATGTGCGCATGC }\end{array}$ \\
\hline sinR-up & $\begin{array}{l}\text { fwd: GCCAAAAGACCTAGATGGTG, } \\
\text { rev: CCTATCACCTCAAATGGTTCGCTGATGTCATCACCTTCCTTGTG }\end{array}$ \\
\hline sinR-down & $\begin{array}{l}\text { fwd: CGAGCGCCTACGAGGAATTTGTATCGGATGACATCCGGGGTATCG, } \\
\text { rev: TAGGAGTTGCTTCTGCAGC }\end{array}$ \\
\hline $\operatorname{degU-up}$ & $\begin{array}{l}\text { fwd: AAGCCCATAAGCTGCAGG, } \\
\text { rev: CCTATCACCTCAAATGGTTCGCTGTATCCGTTTAACACCTTCACG }\end{array}$ \\
\hline degU-down & $\begin{array}{l}\text { fwd: } \\
\text { rev: CAAGCGCCTACGAGGAATTTGTATCGTAAACGACCGGACGCAAGCC, }\end{array}$ \\
\hline spo0A-up & $\begin{array}{l}\text { fwd: TATCAGAGATTCTGCTGCTGGC, } \\
\text { rev: CCTATCACCTCAAATGGTTCGCTGAGCGACAGGCATTCCTGTCC }\end{array}$ \\
\hline spo0A-down & $\begin{array}{l}\text { fwd: } \overline{\text { CGAGCGCCTACGAGGAATTTGTATCGGTTGCGGATAAGCTGAGG, }} \\
\text { rev: GGAAGAACCTGAGACACCG }\end{array}$ \\
\hline kan cassette & $\begin{array}{l}\text { fwd: CAGCGAACCATTTGAGGTGATAGG, } \\
\text { rev: CGATACAAATTCCTCGTAGGCGCTCGG }\end{array}$ \\
\hline spec cassette & 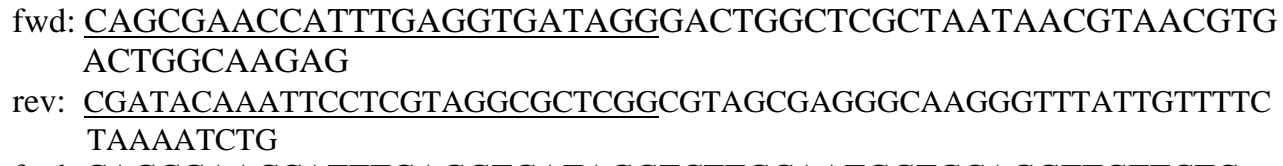 \\
\hline tet cassette & $\begin{array}{l}\text { fwd: CAGCGAACCATTTGAGGTGATAGGTCTTGCAATGGTGCAGGTTGTTCTC, } \\
\text { rev: } \text { CGATACAAATTCCTCGTAGGCGCTCGGGAACTCTCTCCCAAAGTTGATCCC }\end{array}$ \\
\hline liaS(clean) up & $\begin{array}{l}\text { fwd: AGCCGGATCCGAAAGGAGGCGGACACCAGG, } \\
\text { rev: GTTCGTTCTCTCCTTTTTCTTCCGGCTCATACGTACTTCACATCC }\end{array}$ \\
\hline liaS(clean) down & $\begin{array}{l}\text { fwd: CCGGAAGAAAAAGGAGAGAACGAACG, } \\
\text { rev: CCATGAATTCAACCGGGCTGGGAAACGAGG }\end{array}$ \\
\hline
\end{tabular}

\footnotetext{
1 'up' and 'down' refers to localization of the fragment relative to the gene(s) to be deleted. Both fragments are approx. $1 \mathrm{~kb}$ in size and include 20-50 nucleotides of the 5'- or 3'-end of the corresponding gene(s), respectively. 2 'fwd', forward. 'rev', reverse. The (universal) linker sequences used for joining reactions are underlined. Restriction sites in bold.
} 


\section{Results and Discussion}

\section{The liaI promoter $\left(\mathbf{P}_{\text {liaI }}\right)$ is induced without exogenous stimuli at the onset of stationary}

phase. Induction of $\mathrm{P}_{\text {lial }}$ after addition of exogenous stimuli, such as cell wall antibiotics, is well documented (Mascher et al., 2004; Pietiäinen et al., 2005). To investigate if $\mathrm{P}_{\text {lial }}$ is also induced in their absence, we examined expression of a $\mathrm{P}_{\text {lial }}$-lac $\mathrm{Z}$ fusion in the reporter strain BFS2470. This strain harbors an insertion of the vector pMUTIN (Vagner et al., 1998) inside the liaI coding sequence, thereby bringing a promoter-less lacZ gene under control of the liaI promoter (Mascher et al., 2003; Mascher et al., 2004).

To study the $\mathrm{P}_{\text {lial }}$ activity in the absence of external stimuli, strain BFS2470 was grown in LB medium with MLS selection over a period of eight hours and samples were taken every hour from mid-logarithmic to late stationary growth phase. The cells were harvested and $\beta$ galactosidase activity was determined, essentially as described previously (Mascher et al., 2004). The results demonstrate that $\mathrm{P}_{\text {lial }}$ is induced eight- to ten-fold without addition of cell wall antibiotics during transition to stationary phase (Fig. 3.1A, grey bars). This induction is completely LiaR-dependent: no $\mathrm{P}_{\text {lial }}$ activity was observed in the isogenic liaR mutant strain TMB011 (Fig. 3.1A, black bars).

These observations were verified independently by Western analysis in the wild type and an isogenic liaR mutant (strain HB0933). Cells were harvested from both cultures at two time points, two hours before and after transition state (equivalent to the time points 2 and $6 \mathrm{~h}$ in Fig. 3.1A). Total cellular protein was prepared and Western analysis performed with LiaH antibodies. The results are in agreement with the data from the $\beta$-galactosidase assays. LiaH expression is induced in stationary phase in the wild type, but not in the liaR mutant (inset to Fig. 3.1A). Furthermore, induction of liaIH expression was also observed at the transcript level in a wild type $B$. subtilis strain during a chronotranscriptome analysis (see below). While only results from $\beta$-galactosidase assays will be shown for subsequent experiments, all key findings were always independently verified by Western analysis.

Transition state adaptation, which enables B. subtilis to gradually adjust to nutrient limitations, is embedded in one of the best studied bacterial developmental programs, a complex regulatory cascade that ultimately leads to the formation of highly resistant endospores (Errington, 2003; Msadek, 1999; Phillips and Strauch, 2002). It is well known that sporulation (and other transition state phenomena such as production of extracellular enzymes, motility, and biofilm formation) is subject to carbon catabolite repression (Schaeffer et al., 1965; Shafikhani et al., 2003; Stanley et al., 2003): cells grown with high amounts of glucose enter stationary phase without activating the gene expression cascade associated with 
sporulation. Therefore, we repeated the experiment shown in Fig. 3.1 in the presence of increasing glucose concentrations. Addition of glucose to the medium delayed $(0.1 \%$ glucose, grey bars) or even abolished ( $0.5 \%$ glucose, white bars) $\mathrm{P}_{\text {lial }}$-induction, without affecting overall growth rate, onset of stationary phase, or final cell density (Fig. 3.1B). High glucose represses the activation of Spo0A, a key regulator of numerous post-exponential processes including sporulation. The activation of Spo0A results in two major effects on gene expression. First, it leads to the activation of a cascade of sigma factors that ultimately govern the formation of the dormant endospore. Second, Spo0A P represses AbrB which itself is a repressor of numerous genes associated with antibiotic production and resistance (Errington, 2003; Msadek, 1999; Phillips and Strauch, 2002). While addition of glucose has pleiotropic effects on numerous regulatory pathways, this observation can nevertheless be viewed as an indication for a link between LiaRS-dependent gene expression and transition state regulation.

(A)

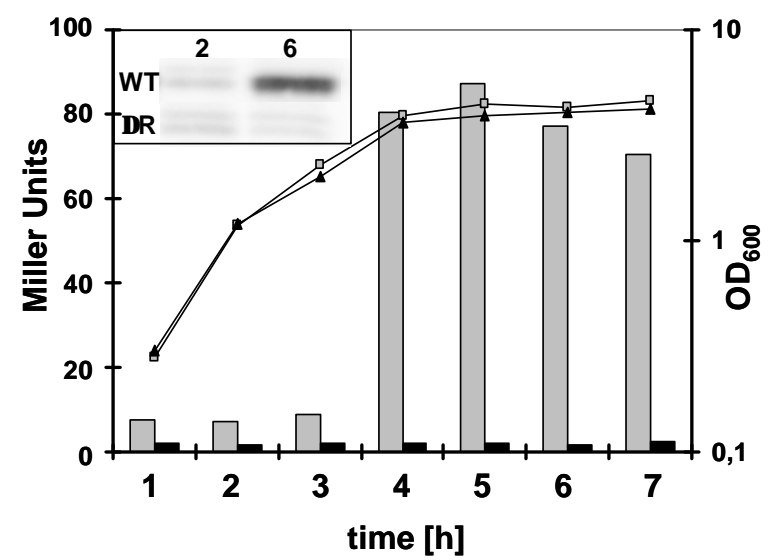

(B)

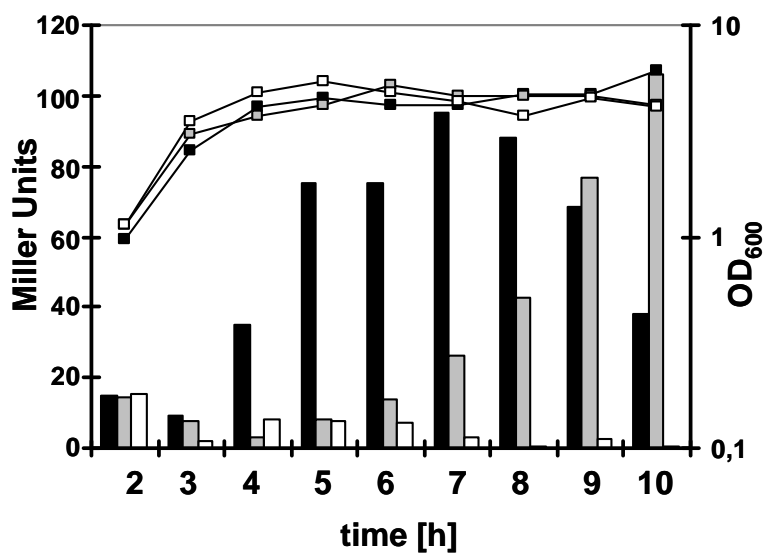

Figure 3.1. Transition state induction of $\mathbf{P}_{\text {lial }}$. (A) $20 \mathrm{ml}$ LB medium (with MLS selection) were inoculated from a fresh mid-logarithmic preculture of strains BFS2470 ('wild type', grey squares or grey bars) and TMB011 (liaR mutant, black triangle and black bars) and incubated at $37^{\circ} \mathrm{C}$ with aeration. Cell density was monitored by measuring the optical density at $600 \mathrm{~nm}\left(\mathrm{OD}_{600}\right)$ at regular intervals, and samples were taken every hour from mid-logarithmic until late stationary growth phase. The cells were harvested, lysed and $\beta$-galactosidase assay was performed as described previously (Mascher et al., 2004). The $\beta$-galactosidase activity, normalized to cell density, is expressed in Miller units (Miller, 1972). The time scale is given in hours, relative to the start of the cultures. The inset shows results of a Western blot analysis of LiaH expression. $20 \mu \mathrm{g}$ total proteins of the wild type (W168) and an isogenic liaR mutant (strains HB0933) were separated by SDS-PAGE. Western blots were performed using CDP-Star (Roche) for chemiluminescent detection, according to the manufacturer's instructions. See the Methods section for details. (B) The same experiment was performed as in Fig. 3.1A. Only the wild type reporter strain BFS2470 was used. The preculture was used to inoculate three different flasks contain $20 \mathrm{ml}$ LB each, without (black symbols/bars), and with the addition of glucose to a final concentration of $0.1 \%$ (grey symbols/bars) and $0.5 \%$ (white symbols/bars). See legend to Fig. 3.1A and the Methods section for experimental details.

A second line of evidence pointing in this direction came from results obtained in a detailed chronotranscriptome study, in which the global gene expression pattern was monitored during a complete growth curve with a resolution of ten minutes (R. Sapolsky, R., P. Iyer, B. 
Caldwell, W. Weyler, G. Chotani, and E. Ferrari, Abstr. 3rd Conf. Functional Genomics of Gram-Positive Microorganisms, abstr. T18, 2005). This study not only verified the transition state induction of liaIH (but not liaGFSR) in a B. subtilis wild type strain at the transcript level, but additionally revealed that expression of lialH coincides with only one other gene, aprE (Eugenio Ferrari, personal communication). This observation can be interpreted in two ways: Either, $\mathrm{P}_{\text {lial }}$ is induced as a result of aprE expression, or both loci are subject to the same regulation.

$\mathbf{P}_{\text {liaI }}$ is repressed by AbrB and activated by Spo0A. To address the first hypothesis, an aprE mutant was constructed by long-flanking homology PCR, and introduced in the $\mathrm{P}_{\text {lial }}$-reporter strain, resulting in strain TMB085 (Table 3.1). No difference of $\mathrm{P}_{\text {lial }}$-activity was observed relative to the wild type reporter strain BFS2470 (data not shown). Therefore, AprE is not involved in $\mathrm{P}_{\text {lial }}$ induction.

The aprE promoter is subject to a complex regulation: one activator (DegU) and three repressors (ScoC, SinR, and $\mathrm{AbrB}$ ) directly bind to the aprE promoter region. The activity of these proteins is modulated by additional proteins, such as Spo0A, SalA and RapG/PhrG (Ogura et al., 2003; Ogura et al., 2004). To analyze a potential role of these proteins in $\mathrm{P}_{\text {lial }}$ activity, mutants in $a b r B, \operatorname{sco} C, \sin R$, and $\operatorname{deg} U$ were constructed and subsequently transferred into the $\mathrm{P}_{\text {lial }}$-reporter strain BFS2470 (Table 3.1). No alterations of $\mathrm{P}_{\text {lial }}$ activity were observed in the $s c o C$, $\sin R$, and $\operatorname{deg} U$ mutant background (data not shown). In contrast, the $a b r B$ mutation in strain TMB087 resulted in an about four-fold elevated basal expression level during logarithmic growth phase, indicating that $\mathrm{AbrB}$ acts as a repressor at $\mathrm{P}_{\text {lial }}$ during that time (Fig. 3.2, black bars). The promoter is still inducible to about wild type levels. Both, the basal promoter activity and the induction of $\mathrm{P}_{l i a I}$ in the $a b r B$ mutant are completely dependent on LiaS-mediated activation of its cognate response regulator, LiaR: a liaS/abrB mutant (TMB330; harboring a clean liaS deletion to avoid polar effects on liaR expression) behaves similar to a liaR mutant, i.e. does not show any $\mathrm{P}_{\text {lial }}$-activity throughout the growth curve (data not shown).

A close regulatory connection between the transition state regulator AbrB and Spo0A, the master regulator of sporulation, is well established: AbrB inhibits Spo0A expression indirectly via $\sigma^{\mathrm{H}}$, contributing to the mechanisms governing temporal control of sporulation initiation. Activated Spo0A, on the other hand, represses $a b r B$ expression, ultimately releasing transition state functions, and $\sigma^{\mathrm{H}}$ expression, from $\mathrm{AbrB}$ repression at the onset of stationary phase (Msadek, 1999; Phillips and Strauch, 2002). As a consequence, mutations in the two genes usually exhibit converse phenotypes on genes subject to their regulation. This 
could also be observed for $\mathrm{P}_{\text {lial }}$ activity: a spoOA mutant (TMB118, Table 3.1) behaved very similar to the liaR mutant in the $\beta$-galactosidase assay, i.e. no detectable $\mathrm{P}_{\text {lial }}$ activity (Fig. 3.2, white bars). These findings were also verified by Western analysis (data not shown). They are in agreement with results from previous transcriptome studies, indicating an indirect Spo0Adependent induction of lialH expression (Fawcett et al., 2000; Fujita et al., 2005; Hamon et al., 2004). Therefore, $\mathrm{P}_{\text {lial }}$ is subject to AbrB repression and Spo0A activation.

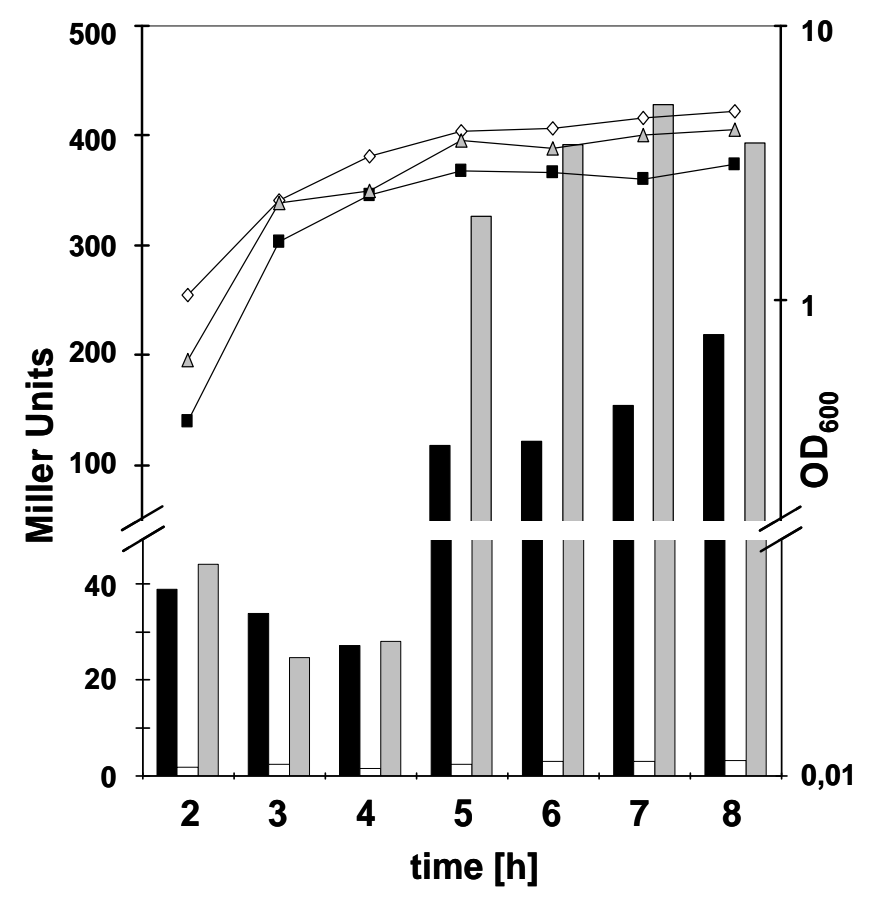

Figure 3.2. Effect of $a b r B$ and spo0A mutations on $P_{\text {lial }}$ activity. Growth and $\beta$-galactosidase acitivity of $\mathrm{P}_{\text {lial }}{ }^{-}$ lac $Z$ fusions were measured for an $a b r B$ (strain TMB087, black squares and black bars), a spo0A mutant (strains TMB118, white diamonds and white bars), and an $a b r B / s p o 0 A$ mutant (strain TMB209, grey triangles and grey bars), respectively. The experiment was performed as described in the legend to Figure 3.1A. The scale on the $y$ axis was split for reasons of clarity.

Spo0A activates $\mathbf{P}_{\text {lial }}$ indirectly through AbrB. The loss of transition state induction of $\mathrm{P}_{\text {lial }}$ in the spo0A mutant raised the question of how Spo0A affects its promoter activity. The $a b r B$ gene is known to be under the direct negative control of Spo0A, and AbrB acts as a repressor of a set of genes that are switched during transition state (Strauch et al., 1990; Strauch et al., 1989). Therefore, Spo0A activation of $\mathrm{P}_{\text {lial }}$ could be an indirect effect due to the lack of Spo0A-dependent repression of $a b r B$. Alternatively, Spo0A itself could be responsible for the expression of genes that ultimately provide the stimulus that is sensed by the LiaRS TCS at the onset of stationary phase. To distinguish between the two possibilities, a mutant lacking both genes, $a b r B$ and $s p o 0 A$, was constructed and introduced into BFS2470, resulting in strain TMB209. This mutant showed a $\mathrm{P}_{\text {lial }}$ induction pattern comparable to the $a b r B$ mutant, i.e. an elevated basal level of $\mathrm{P}_{\text {lial }}$ activity during logarithmic growth, and induction at the onset of 
stationary phase (Fig. 3.2). Interestingly, the maximum $\mathrm{P}_{\text {lial }}$ activity was reproducibly higher by a factor of two than in the $a b r B$ mutant (Fig. 3.2, grey bars). The reason for this behavior remains elusive, so far. But the results clearly demonstrate that Spo0A indirectly modulates $\mathrm{P}_{\text {lial }}$ activity by repressing $a b r B$ expression.

AbrB directly binds $\mathbf{P}_{\text {liaI }}$. The transition state regulator AbrB directly regulates (mostly represses) the expression of over 50 genes, with many additional loci being subject to indirect AbrB control (Phillips and Strauch, 2002). Despite in-depth knowledge on numerous AbrB binding sites, no consensus sequence has been identified for chromosomal sites of interaction. It has been hypothesized that AbrB recognizes a conserved three-dimensional DNA structure, rather than specific base pairs, in the promoter regions of its target genes (Bobay et al., 2004; Phillips and Strauch, 2002; Xu and Strauch, 1996). DNase I footprinting analysis of the liaI promoter region demonstrates that AbrB protects a DNA region of about 25 base pairs (from 40 to -14 ), with weaker protection occurring further downstream (from -11 to about +10 ) (Fig. 3.3). In contrast, the AbrB paralog Abh does not bind the $\mathrm{P}_{\text {lial }}$ region under these conditions (Fig. 3.3). We conclude that $\mathrm{AbrB}$ repression of $\mathrm{P}_{\text {lial }}$ occurs through direct binding of the repressor within the promoter sequence, thereby preventing transcription initiation.

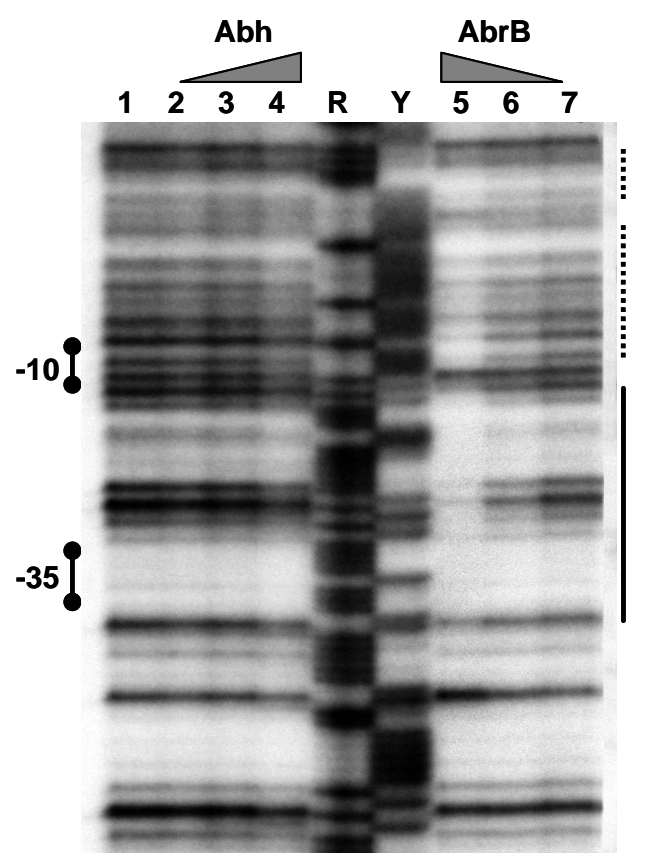

Figure 3.3. DNase I footprinting analysis of AbrB binding to $\mathbf{P}_{\text {lial }}$. DNase I footprinting was performed as

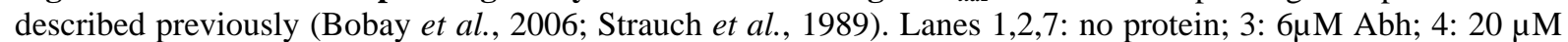
Abh; 5: $20 \mu \mathrm{M}$ AbrB; 6: $6 \mu \mathrm{M}$ AbrB. AbrB binding reactions were performed at $\mathrm{pH}$ 8; Abh binding at $\mathrm{pH} 7$ (the optimal pH's for each protein's binding (Bobay et al., 2006)). R, Y = Maxam-Gilbert purine and pyrimidine chemical sequencing reactions. The DNA target was a $287 \mathrm{bp}$ fragment containing the liaI promoter region (positions -159 to +128 ) end-labeled on the template strand. Solid vertical line on right $=$ seemingly stronger AbrB binding region; dashed lines on right $=$ seemingly weaker protection region due to AbrB binding. -35 and 10 regions of the liaI promoter are indicated on the left. 
LiaR is sufficient for $\mathbf{P}_{\text {liaI }}$ induction. Induction of $\mathrm{P}_{\text {liaI }}$ under conditions of cell envelope stress is strictly LiaR-dependent (Mascher et al., 2003). To determine if AbrB or Spo0A are also necessary for $\mathrm{P}_{\text {lial }}$-dependent transcription in the presence of external stimuli, such as bacitracin, we performed $\beta$-galactosidase assay and Western analysis in the wild type, and isogenic liaR, $a b r B$, and spoOA mutants from cells harvested mid-exponentially with and without the presence of bacitracin (final concentration $50 \mu \mathrm{g} / \mathrm{ml}$ ). With the exception of the liaR mutant, all strains were inducible to comparable levels, demonstrating that LiaR alone is sufficient for bacitracin-induced $\mathrm{P}_{\text {lial }}$-activity (Fig. 3.4, and data not shown). These experiments also demonstrate that strong inducers such as bacitracin (3,000 to 4,000 Miller units from $\mathrm{P}_{\text {lial }}$-lac $Z$ in $\beta$-galactosidase assays) can completely overcome $\mathrm{AbrB}$ repression, whereas the endogenous transition state induction (100-200 Miller units) can be suppressed by increased cellular levels of $\mathrm{AbrB}$ as present in the spoOA mutant. The mechanism by which fully activated LiaR (i.e. in response to bacitracin stress) seemingly "overrides" AbrB control is completely unknown. We can only speculate that the affinity of phosphorylated LiaR for its target promoter somehow is higher than that of AbrB, whereby activated LiaR seems to be able to displace bound $\mathrm{AbrB}$ and initiate $\mathrm{P}_{\text {lial }}$-dependent transcription in the presence of strong inducers.

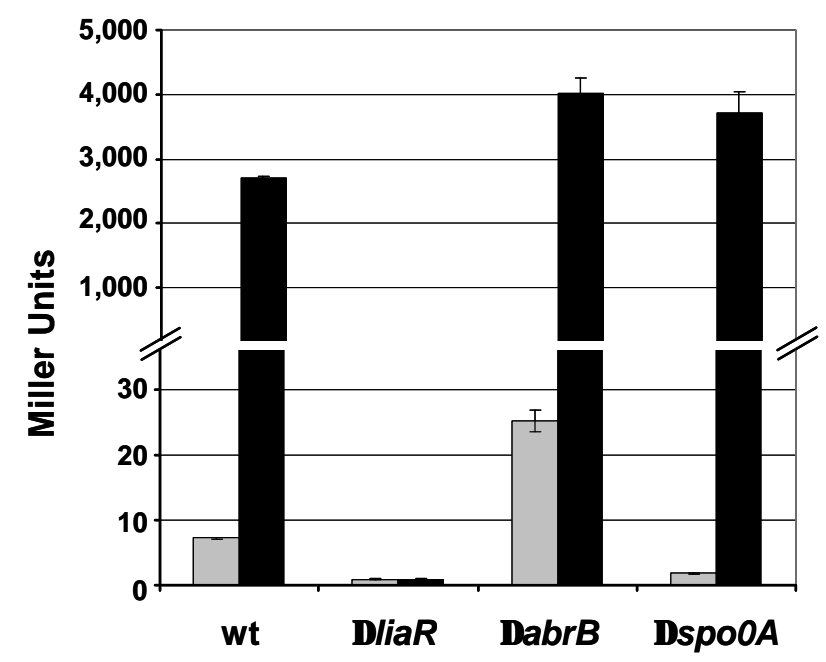

Figure 3.4. Bacitracin induction of $P_{\text {liaI }}$ in strain BFS2470 (wt), and isogenic liaR (strain TMB011), abrB (strain TMB087), and spo0A (strain TMB118) mutants. All strains were grown in LB medium with MLS selection to mid-log. The cultures were split, one half induced with bacitracin (final concentration $50 \mu \mathrm{g} / \mathrm{ml}$; black bars), the other half remained as uninduced control (grey bars). Incubation was continued for $30 \mathrm{~min}$, before $2 \mathrm{ml}$ of culture were harvested. The cells were lysed and $\beta$-galactosidase assay was performed as described previously (Mascher et al., 2004). The $\beta$-galactosidase activity, normalized to cell density, is expressed in Miller units (Miller, 1972). The scale on the $y$-axis was split for reasons of clarity. 
The nature of the endogenous stimulus responsible for transition state induction of $\mathbf{P}_{\text {liaI }}$ remains obscure. The known induction of $\mathrm{P}_{\text {lial }}$ by cell wall antibiotics led us to hypothesize that induction in early stationary phase might be due to a secreted antibiotic synthesized by $B$. subtilis itself (Stein, 2005). To address this question, induction experiments with spent medium were performed. The wild type reporter strain BFS2470 was grown in LB medium with MLS selection until two hours after transition state (corresponding to $t=6 \mathrm{~h}$ in Fig. 3.1A). A sample of the culture was harvested to check for $\mathrm{P}_{\text {lial }}$-induction. The cells were removed from the remaining culture and the spent medium was directly used to resuspend fresh mid-logarithmic cells $(\mathrm{t}=2 \mathrm{~h})$ that were incubated in parallel. Additionally, cells were resuspended in fresh prewarmed LB medium, with and without addition of bacitracin (final concentration $50 \mu \mathrm{g} / \mathrm{ml}$ ), as a positive and negative control, respectively. After further incubation at $37^{\circ} \mathrm{C}$ for $30 \mathrm{~min}$, the cells were harvested. The results from $\beta$-galactosidase assays are shown in Fig. 3.5. The cells from the stationary phase culture showed the expected $\beta$-galactosidase activity. Resuspension of mid-log cells in fresh LB medium only resulted in the normal background activity of about 10 Miller units, while resuspension in LB medium supplemented with bacitracin gave the typical strong $\mathrm{P}_{\text {lial }}$-response (about 2,000 Miller units). In contrast, no induction was observed when mid-logarithmic cells were resuspended in spent medium (Fig. 3.5, last bar). These results indicate that $\mathrm{P}_{\text {lial }}$ is not induced by a secreted compound produced by $B$. subtilis itself. But we cannot rule out the possibility that the inducing antibiotic is not released from the cells in sufficient amounts in the medium to be detectable in our conditioned medium experiments. For example, a prerequisite for the biological potency of many cationic antimicrobial peptides is their binding to the overall negatively charged cell envelope, and modulating this net charge is an important resistance mechanism of many gram-positive bacteria against their activity (Kovács et al., 2006; Peschel et al., 1999). Conversely, one could imagine that such an antibiotic, produced by B. subtilis itself, might be retained to a certain degree by the negatively charged cell wall. Therefore, while $\mathrm{P}_{\text {lial }}$-induction could then be readily measured in the antibiotic-producing stationary phase culture, this inducer would not necessarily accumulate in the medium in amounts sufficient to activate $\mathrm{P}_{\text {lial }}$ in resuspended mid-logarithmic cells.

We also attempted to identify potential genetic determinants involved in generating the endogenous stimulus by applying transposon mutagenesis in the $\mathrm{P}_{\text {lial }}$-lacZ reporter strain BFS2470 and screening for blue colonies, indicative for increased $\mathrm{P}_{\text {lial }}$ activity. Two independent approaches were used, in-vivo transposon mutagenesis, based on the established mini-Tn10 system encoded on plasmid pIC333 (Steinmetz and Richter, 1994), and a newly 
developed in-vitro system, based on Tn7 (Peters and Craig, 2001). The latter also allows gainof-function mutagenesis screens, due to the presence of an outward-facing, xylose-inducible promoter. We readily isolated mutants with transposon insertions in liaF, the known negative regulator of the LiaRS systems (Jordan et al., 2006). In addition, we also recovered insertions in the export pump of a putative bacteriocin, indicating that endogenous peptides produced by B. subtilis can induce the LiaRS system (Butcher and Helmann, unpublished). However, strains lacking the ability to produce this bacteriocin still induce $\mathrm{P}_{\text {lial }}$ upon entry into stationary phase (data not shown). Therefore, the nature of this endogenous stimulus remains unknown.

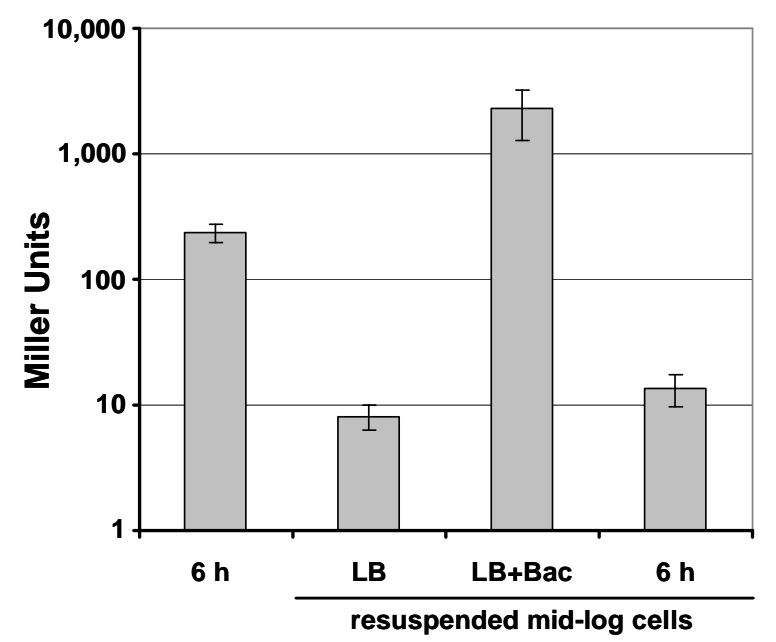

Figure 3.5. $\mathbf{P}_{\text {lial }}$-induction experiments with spent medium. A "donor" culture of strain BFS2470 was grown until two hours after transition state ("6h") and $\mathrm{P}_{\text {lial }}$-activity was determined to ensure inducing conditions (first bar). The corresponding culture supernatant was used after removing of cells by centrifugation, to induce cells from a second mid-logarithmic culture (fourth bar). As a control, mid-log cells were also resuspended in prewarmed fresh LB medium without (LB, second bar) and with addition of bacitracin (final concentration 50 $\mu \mathrm{g} / \mathrm{ml}$; third bar), as a negative and positive control. The resuspended mid-log cells were incubated for another $30 \mathrm{~min}$ at $37^{\circ} \mathrm{C}$, before the cells were harvested and used in a $\beta$-galactosidase assay. The $\beta$-galactosidase activity, normalized to cell density, is expressed in Miller units (Miller, 1972). A log-scale was used on the $y$ axis for reasons of clarity.

\section{Conclusions}

Based on the results of previous studies and those presented herein, the intrinsic induction of liaIH expression at the onset of stationary phase is tightly regulated and delicately balanced by five proteins - LiaR, LiaS, LiaF, AbrB, and SpoOA - to allow an appropriate cellular response at the right time. The interactions and hierarchy of these regulators is illustrated in the model in Fig. 3.6. During logarithmic growth in the absence of cell envelope stress, the 
LiaRS TCS is kept inactive by the LiaF regulator (Jordan et al., 2006). Furthermore, the transition state regulator $\mathrm{AbrB}$ represses any residual $\mathrm{P}_{\text {lial }}$ activity by binding to a DNA fragment that includes the -35 region and reaches the -10 region, thereby serving as a roadblock that efficiently prevents transcription initiation (Fig. 3.6, right-hand side). At the onset of stationary phase, increasing levels of phosphorylated Spo0A, the master regulator of sporulation, inhibit $a b r B$ expression (Strauch et al., 1990), thereby releasing $\mathrm{P}_{\text {lial }}$ from its repression. At about the same time, an unidentified stimulus leads to the activation of the histidine kinase LiaS and/or its release from LiaF repression. This, in turn, leads to the activation of the cognate response regulator LiaR, which interacts with its binding site (an imperfect inverted repeat of seven nucleotides with four nucleotides spacing) (Jordan et al., 2006), ultimately resulting in induction of liaIH expression (Fig. 3.6, left-hand side).

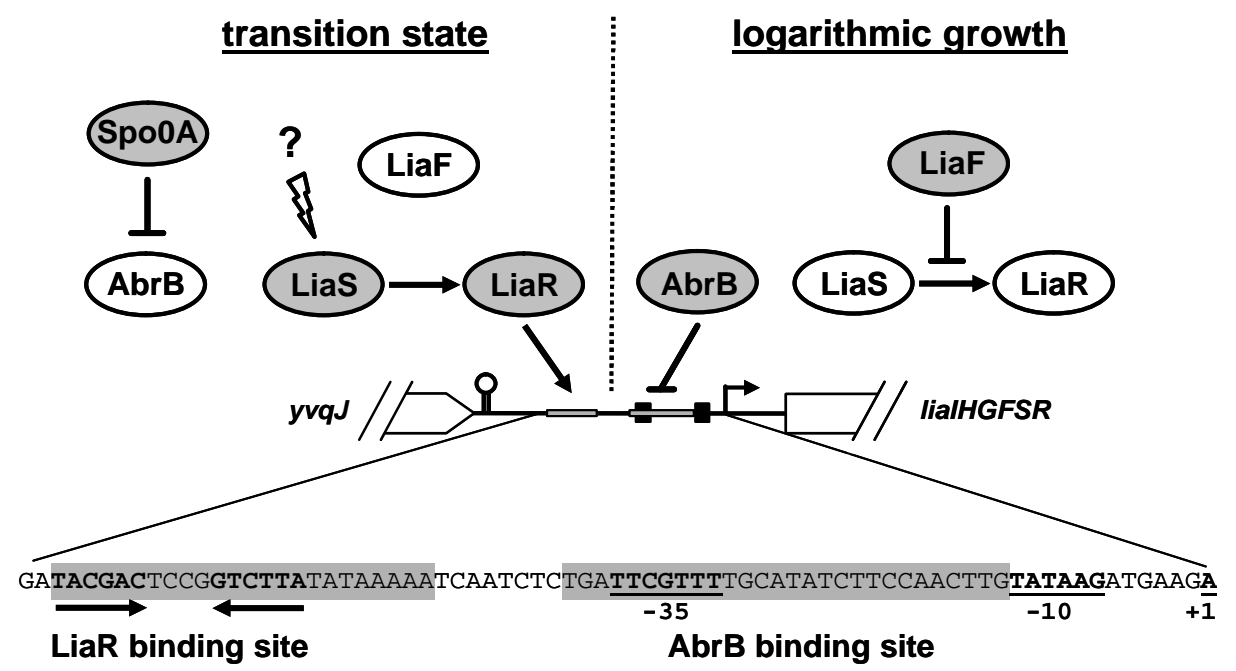

Figure 3.6. Model for the transition state regulation of $\mathbf{P}_{\text {lial }}$ activity. The situation during logarithmic growth and transition state are shown on the right and left, respectively, separated by the dotted line. Regulatory proteins involved are named and circled. Grey backgrounds indicate activity, white inactivity. Arrows indicate activation, $\mathrm{T}$-shaped lines repression. The genomic context of the liaI promoter region and its important features are schematically shown in the middle, the relevant sequence is detailed out below. The identified regulator binding sites are highlighted in grey. The inverted repeat in the LiaR binding site is indicated by the two arrows below. The promoter sequence and transcriptional start is highlighted in bold and underlined. See text for details.

Previous studies identified numerous agents that are able to induce LiaRS-dependent gene expression. While some of these compounds, especially cell wall antibiotics that interfere with the lipid II cycle (i.e. bacitracin, nisin, ramoplanin or vancomycin) elicit a strong response (Mascher et al., 2004), the biological relevance of these observations remains obscure, since the LiaRS system is not involved in mediating resistance against any of these inducers (unpublished results). The induction of $\mathrm{P}_{\text {lial }}$ at the onset of stationary phase - while being significantly weaker (about 10-15 fold, Fig. 3.1A, compared to 50-200 fold in case of 
strong inducers (Mascher et al., 2004), as also shown in Fig. 3.4) - is therefore an important observation. While a very high dynamic potential and strength of LiaRS-dependent gene expression could be demonstrated by the exogenous addition of cell wall antibiotics (Mascher et al., 2004), this situation does not necessarily reflect the "natural" condition for the activation of LiaRS-dependent signal transduction. The different sites and modes of action of these known inducers of the LiaRS system, together with the unique domain architecture of the sensor kinase LiaS, argue against a direct binding of these drugs to the input domain of LiaS (Mascher, 2006b). Identification of the true sensory input of the LiaRS system, while being a big challenge, is therefore a prerequisite to understand the difference in LiaRdependent gene expression observed in this study.

\section{Acknowledgements}

We thank Eugenio Ferrari (Genencor) for sharing unpublished results from their chronotranscriptome study, Zoltan Pragai for the gift of strain BFS2470, and Jörg Stülke, in whose laboratory this research was conducted. This work was financially supported by grants GM46700 (to M.A.S.) and GM47446 (to J.D.H.) from the National Institutes of Health, and grants from the Deutsche Forschungsgemeinschaft (MA3269) and the Fonds der Chemischen Industrie (to T.M.). 


\section{Chapter 4: Discussion}

The LiaRS TCS is a part of the regulatory network that orchestrates CESR in B. subtilis (chapter 1). It responds to the external presence of cell wall antibiotics that interfere with the lipid II cycle, such as bacitracin, ramoplanin, vancomycin, or cationic antimicrobial peptides (Mascher et al., 2004; Pietiäinen et al., 2005), without being involved in AB resistance. It is also induced by alkaline shock, detergents, ethanol, phenol, organic solvents, and secretion stress, albeit to a lesser extent (Hyyryläinen et al., 2005; Mascher et al., 2004; Petersohn et al., 2001; Pietiäinen et al., 2005; Tam le et al., 2006; Wiegert et al., 2001). While its physiological role is still unclear, the data presented in this thesis lead to a detailed understanding of LiaRS-mediated signal transduction.

LiaF could be identified as an essential protein in LiaRS-mediated signal transduction (chapter 2). Without cell encelope stress, LiaF acts as a repressor of LiaRS-dependent gene expression by preventing LiaS-mediated phosphorylation of LiaR. Deletion of liaF leads to constitutive induction of the LiaR target promoters. For the repressory function of LiaF, the cytoplasmic located C-terminal part of the protein is essential. Mutants with in-frame deletions in this region behave like a liaF deletion mutant (Fig. 2.2 and 2.3). Therefore, LiaF together with LiaRS constitute a CES-sensing three-component system.

The LiaR-binding site was determined as a 7-4-7 inverted repeat in the promoter region of liaI homologs in the Firmicutes bacteria (Fig. 2.5). In B. subtilis, mutants that harbour exchanges of highly conserved bases within this motiv no longer respond to the presence of bacitracin (Tab. 2.4). So far, two target promoters could be verified: $\mathrm{P}_{\text {lial }}$ and $\mathrm{P}_{y h c Y}$ (Fig. 2.1 and 2.7). A third LiaR-binding site was also identified by comparative genomics upstream of yozJ (chapter 2) and transcriptome studies identified another LiaR-dependent gene, $y d h E$ (see below).

In addition to the response to cell wall antibiotics that interfere with the lipid II cycle and several other stresses, the liaI promoter is induced without external stimuli during transition state (chapter 3). This activity is embedded in the complex regulatory cascade governing adaptation at the onset of stationary phase. During logarithmic growth the liaI promoter is directly repressed by the transition state regulator $\mathrm{AbrB}$, which binds the DNA region of $\mathrm{P}_{\text {lial }}$ between -40 and -14 (Fig. 3.3). It could be demonstrated that Spo0A, the master regulator of sporulation, activates $\mathrm{P}_{\text {lial }}$ indirectly by repressing $a b r B$ expression (Fig. 3.2). Additional links between LiaRS and Spo0A are suggested by data from the literature, as will be discussed below. 
Here, we will put the findings derived from this thesis in the context of recent findings and the data from the available literature. The first paragraph will address the LiaR regulon and the physiological role of LiaFSR-dependent gene expression. In the second paragraph, the role of accessory proteins like LiaF for two-component signal transduction will be described. The mechanism of stimulus perception by the LiaS-LiaF sensory unit will be discussed in the third paragraph. In the fourth paragraph, the importance of the stoichiometry between LiaF, LiaS and LiaR will be addressed. The last paragraph will engage in the discovery of bistable behaviour of $\mathrm{P}_{\text {lial }}$ induction in transition state.

\section{The LiaR regulon}

In addition to the published results (Jordan et al., 2006) - i.e. LiaR-dependent expression of liaIH and $y h c Y Z y h d A$ - we could identify one additional target locus of LiaR, the $y d h E$ gene, in a DNA microarray analysis in collaboration with Georg Homuth and Ulrike Mäder (Greifswald), by comparing the global transcription pattern of the wild type and isogenic liaF and liaR mutants, representing the "ON" and "OFF" state of the LiaRS-system, respectively. The expression of $y d h E$ is upregulated about 15 -fold (Fig. 4.1A) in the liaF mutant, an induction comparable to the $y h c Y Z-y h d A$ operon. This induction is much weaker than that observed for liaIH, and both loci are not inducible by bacitracin in the wild type strain (Fig. 4.1B). In B. subtilis, LiaR-dependent induction of the $y h c Y Z-y h d A$ operon by bacitracin was only observed in a liaH mutant (Mascher et al., 2003), while it was already strongly upregulated in a B. licheniformis wild type strain (Wecke et al., 2006). We identified a putative LiaR-binding site upstream of the $y h c Y$ promoter in both B. subtilis and $B$. licheniformis (Jordan et al., 2006). A LiaR-dependence of the $y d h E$ promoter was already indicated by a previous transcriptome study (Kobayashi et al., 2001), and we identified a weakly conserved potential LiaR binding site in the promoter region of the $y d h E$ gene (Fig 4.2), which still needs to be verified experimentally. The domain architecture of the corresponding LiaR-target proteins is illustrated in Fig. 4.1C.

To understand the physiological role of LiaFSR-dependent signal transduction, it is important to get deeper insights into the function of its target proteins. While this is subject to ongoing studies, some speculations can be drawn from preliminary data and will be presented below.

The liaIH operon is the primary target of LiaRS-dependent signal transduction, as mentioned above. In collaboration with Michael Hecker and Birgit Voigt (Greifswald), we were able to verify this on the protein level by two-dimensional gel electrophoresis of the cytoplasmic 
proteome, using cell extracts prepared from the wild type W168 - with and without addition of bacitracin - and isogenic liaR ("OFF") and liaF ("ON") mutants. LiaH was the most abundant cytosolic protein in the liaF mutant, and identified in five independent spots, indicative for posttranslational modifications, while it was hardly detectable in the wild type (without bacitracin induction) and absent in the liaR mutant (Falk Kalamorz, diploma thesis). The appearance of multiple LiaH-spots is presumably due to different phosphorylation states of the protein, since it was recently demonstrated that PrkC phosphorylates $\mathrm{LiaH}$ in vitro (Pietack et al., unpublished).

(A)

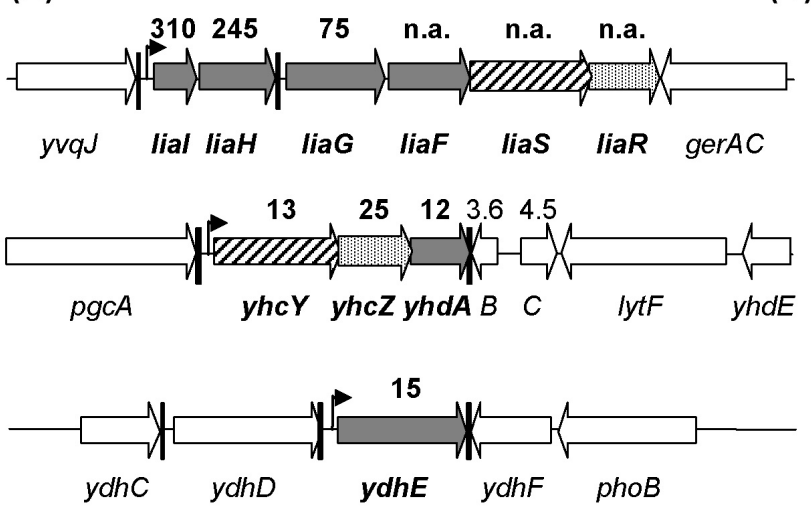

(C)

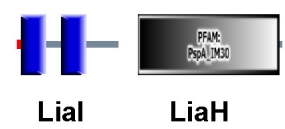

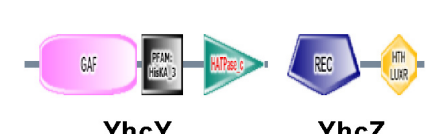

YhcY
(B)

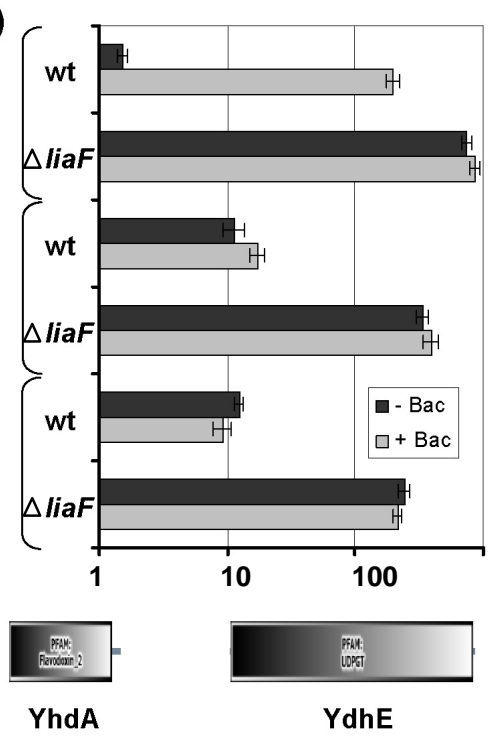

Figure 4.1. The LiaRS regulon. (A) Genomic context of LiaR-target genes. The regions are drawn to scale, the line represents $7 \mathrm{~kb}$. Target genes are indicated by bold gene names and filled arrows; hatched arrows $=$ histidine kinases, dotted arrows $=$ response regulators, grey arrows $=$ other target genes of unknown function. White arrows indicate flanking genes. The numbers above are fold-induction of a liaF mutant relative to its isogenic wild type, derived from DNA microarray studies; n.a. = not available due to the liaF::kan insertion. (B) Verification of LiaR-dependent promoter activity by $\beta$-galactosidase assays. The LiaR-dependent promoter regions used are highlighted in Fig. 2A. The three brackets represent (from top to bottom) $\mathrm{P}_{\text {lial }}, \mathrm{P}_{y h c}$, and $\mathrm{P}_{y d h E^{-}}$ lacZ-fusions, measured with (grey bars) and without (black bars) addition of bacitracin in the wild type (W168) and an isogenic liaF mutant. Scale on the $x$-axis is in Miller units. (C) Domain architecture of LiaR-target proteins, derived from the SMART database at http://smart.embl-heidelberg.de/. Blue vertical bars represent putative transmembrane regions, labelling of all other domains according to SMART or Pfam nomenclature. See text for details.

LiaH belongs to the PspA-IM30 protein family of phage-shock proteins, the name derived from PspA of E. coli. The latter is induced by various stress conditions such as filamentous phage infection (hence the name), heat shock, osmotic shock, exposure to organic solvents and proton ionophores as well as long incubation under alkaline conditions (Brissette et al., 1990; Kobayashi et al., 1998; Weiner and Model, 1994). This inducer spectrum shows some overlap with the known inducers of liaIH expression, which include organic solvents and alkaline shock (Mascher et al., 2004; Wiegert et al., 2001). PspA exhibits a dual function that 
is linked to two different cellular locations (Brissette et al., 1990; Kleerebezem and Tommassen, 1993): Peripherally bound to the inner surface of the cytoplasmic membrane, PspA is involved in the maintenance of cell membrane integrity (Darwin, 2005; Kleerebezem et al., 1996). As a free cytosolic protein, it inhibits the AAA+ enhancer protein PspF through protein-protein interactions (Adams et al., 2003; Bordes et al., 2003; Dworkin et al., 2000). Members of the PspA/IM30 protein family are therefore referred to as AAA+ adaptor proteins (Hankamer et al., 2004). Preliminary data indicate that LiaH is bound to the inner surface of the cytoplasmic membrane via LiaI (data not shown). LiaI is a small hydrophobic protein with two deduced transmembrane helices, encoded directly upstream of liaH. The finding that LiaH can be bound to the cytoplasmic membrane like PspA leads to the hypothesis that LiaH like PspA might be involved in the maintenance of cell membrane integrity (Kobayashi, 2007).

Ongoing phenotypical analyses revealed that LiaH plays some role in cellular survival under conditions of severe envelope stress, exhibited by the presence of some cell wall antibiotics such as fosfomycin or cephalexin, without conferring antibiotic resistance against them: while the minimal inhibitory concentration only marginally increased in the liaF mutant (if at all), the survival rate when grown in the presence of inhibitory antibiotic concentrations (determined as colony forming units in serial dilution spot tests) differed by some orders of magnitude, with either an increase in survival for the liaF mutant, or decreased survival rates for the liaH mutant (Diana Hoyer, diploma thesis).

For both, E. coli PspA and cyanobacterial Vipp1, the formation of large oligomeric ring-like structures was demonstrated (Aseeva et al., 2004; Hankamer et al., 2004). Transmission electron microscopic studies revealed that purified LiaH also forms rings with a nine-fold rotational symmetry in vitro (Falk Kalamorz, diploma thesis). Gel filtration experiments verified the formation of one homogenous LiaH oligomeric complex with total molecular weight of more than $1,000 \mathrm{kDa}$ (Kirstein, personal communication). These observations indicate that LiaH might also function as an AAA+ adaptor protein.

There are strong indications that there is a link between LiaH and protein secretion. In E. coli CESR is defined as counteracting misfolded proteins in the periplasm (Ruiz and Silhavy, 2005). E. coli PspA is involved in maintenance of pmf (Darwin, 2005), which solely (Tat) or partially (Sec) drives protein secretion in this organism (DeLisa et al., 2004; Economou, 1999). Moreover, it was demonstrated that PspA overexpression in E. coli increases Tatdependent protein secretion (DeLisa et al., 2004). Recently, it was shown that PspA overexpression in Streptomyces lividans improves both Sec- and Tat-dependent protein 
secretion (Vrancken et al., 2007). Along those lines, the secreted proteins from B. subtilis wild type cultures, and liaH and liaF mutants - lacking or overexpressing LiaH, respectively - were prepared. SDS-PAGE and 2D-gels revealed numerous significant differences in the protein patterns, clearly indicating a role of LiaH in protein secretion (Diana Hoyer, diploma thesis).

The $y h c Y Z-y h d A$ operon encodes a TCS (YhcYZ) and a glycosyltransferase (YhdA). In addition to our findings, the LiaRS-dependent expression of $y h c Y Z$ was also indicated by a comprehensive microarray analysis of TCS (Kobayashi et al., 2001), based on the overexpression of the response regulator in the absence of its cognate histidine kinase. YhcY is a soluble histidine kinase that harbors a cytoplasmic GAF input domain. These highly conserved and widely (from bacteria to mammals) distributed domains bind cyclic nucleotides, primarily cGMP, but also cAMP, indicative for monitoring the cellular energy state (Anantharaman et al., 2001; Hurley, 2003; Martinez et al., 2002). So far, most bacterial GAF domains investigated are part of (cyano-)bacteriophytochromes, where they are involved in heme binding (Gao et al., 2007; Ivleva et al., 2006; Mutsuda et al., 2003; Zhang et al., 2006). The only histidine kinase exclusively containing GAF input domains that has been analyzed so far is DosS from Mycobacterium tuberculosis. It is inducible by hypoxia, $\mathrm{H}_{2} \mathrm{O}_{2}$, NO and ethanol (Kendall et al., 2004; Sherman et al., 2001; Voskuil et al., 2003). Again, biochemical studies demonstrated that at least one of its two GAF domains binds heme (Sardiwal et al., 2005). So far, the biological function of the YhcYZ TCS is unknown. A close homolog, YhcSR, has recently been described as an essential TCS in Staphylococcus aureus, potentially involved in envelope stress response (Sun et al., 2005). In contrast, deletion mutants of $y h c Y Z$ are perfectly viable in B. subtilis.

YhdA is a putative NADPH-dependent FMN reductase, with strong homology to an azoreductase of Bacillus sp. OY1-2 (Suzuki et al., 2001). A structural homolog, YLR011wp from Saccharomyces cerevisiae, was shown to exhibit a NAD $(\mathrm{P}) \mathrm{H}$-dependent FMN reductase and a strong ferricyanide reductase activity (Liger et al., 2004).

$\underline{\text { YhE }}$ is a putative UDP-glucoronosyl or UDP-glucosyl transferase, with homology to macrolide glycosyltransferases (Hernandez et al., 1993) and zeaxanthin glucosyltransferases (enzymes involved in carotenoid biosynthesis). UDP-glucosyl transferases have been found in plants, animals, fungi and bacteria. They use UDP-activated sugar moieties as the sugar donor 
and small molecules such as flavonoids, alkaloids, antibiotics and plant hormones as the sugar acceptors.

In summary, we were able to identify four (potential) LiaR-binding sites in the genome of $B$. subtilis (Fig. 4.2). The expression of lial, $y h c Y$ and $y d h E$ increases in a liaF mutant, the "ON" state of the LiaRS TCS. The LiaR-binding sites upstream of these three genes are in about the same distance (between 75 and $85 \mathrm{bp}$ ) from the ATG start codon. The fourth putative LiaRbinding site is located 208 bp upstream of yozJ (Fig. 4.2). This is an unusual long distance, twice as much as for any of the other known LiaR-binding sites (Jordan et al., 2006). Furthermore, we failed to detect an induction of yozJ expression after treatment with bacitracin and in a liaF mutant using a $\mathrm{P}_{y o z}-\beta$-galactosidase fusion. Two possibilities can be considered for this LiaR-binding: Either LiaR is not able to bind this DNA region or LiaR can bind but the expression of yozJ is not induced. Since the intergenic region upstream of yozJ is relatively large, a noncoding small RNA could be located within. This hypothesis is supported by the presence of a strong stem loop structure (Fig 4.2B). But this is highly speculative and needs further investigation.

(A)

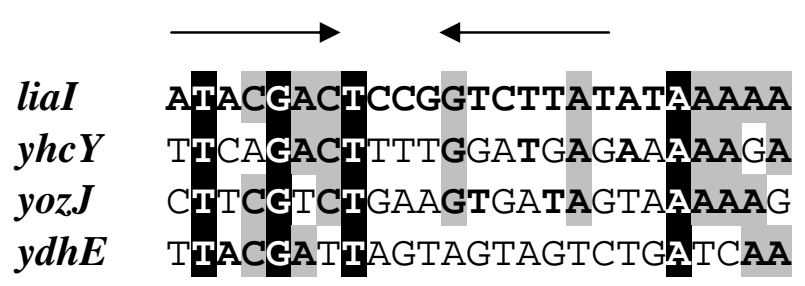

(B)

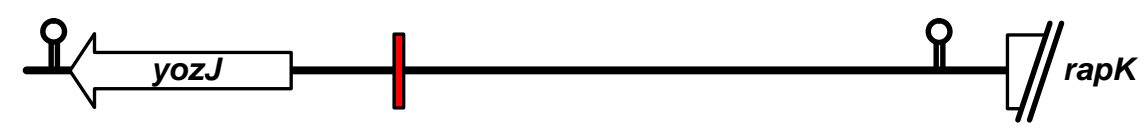

Figure 4.2. Putative LiaR-binding sites in B. subtilis. (A) Alignment of the four LiaR-binding sites. Nucleotides conserved in all four sequences are highlighted as white letters on black ground. Nucleotides conserved in three of four sequences are highlighted in grey. Nucleotides that correspond to the LiaR-binding site upstream of liaI are given in bold letters. The inverted repeat is marked with arrows. (B) Genomic context of the LiaR-binding site upstream of yozJ. The binding site is shown in red and orientated towards rapK.

A LiaR-dependent induction of $\mathrm{P}_{y h c Y}$ and $\mathrm{P}_{y d h E}$ could only be observed in a liaF mutant, an artificially situation where the LiaRS TCS is constitutively active. So far, the lia operon is the only LiaR-dependent locus that is induced under conditions of cell envelope stress in the wildtype (Fig. 4.1). Therefore, we postulate that liaIH is the only relevant target of the LiaRS TCS. 
Induction of $\mathrm{P}_{y h c Y}$ in a liaF mutant might be due to the significant similarity of the RR LiaR and YhcZ. Both belong to the NarL/FixJ class of response regulators (Galperin, 2006), and they are highly homologous to each other, even within the DNA binding domain. Maybe the binding site upstream of $y h c Y$ is primarily recognized by YhcZ, but because of the similar DNA binding domains of the regulators, activated LiaR is able to bind when it is extremly overexpressed from a plasmid (Kobayashi et al., 2001) or in a liaF mutant (Jordan et al., 2006). Again, these possibilities are currently under investigation.

\section{Accessory proteins for stimulus perception and control of TCS function: Bacterial three- component systems}

A major finding of this thesis was the identification of LiaF as a strong inhibitor of the LiaRS TCS. LiaF is an accessory protein of the LiaRS TCS essential for its function and probably for stimulus perception. While the presence of additional proteins is the exception rather than the rule for two-component signal transduction, a number of TCS exist where stimulus perception is not mediated by the histidine kinase alone, but through or together with an additional protein ("accessory sensory protein"). Such accessory sensor proteins can be periplasmic, membrane-bound or cytoplasmic proteins (see Tab. 4.1). Examples for periplasmic solute-binding proteins are ChvE from Agrobacterium tumefaciens (Shimoda et al., 1993), LuxQ from Vibrio harveyi (Bassler et al., 1994) and BctC from Bordetella pertussis (Antoine et al., 2005). Soluble accessory proteins only occur in conjunction with cytoplasmic-sensing HK. Examples are the [Ni-Fe]-Hydrogenase HoxBC from Ralstonia eutropha, which functions as the $\mathrm{H}_{2}$-sensor (Kleihues et al., 2000; Lenz and Friedrich, 1998; Schwartz et al., 1998), and FixT from Sinorhizobium meliloti that functions as an anti-kinase by interacting with the C-terminal transmitter domain of FixL, thereby interfering with autophosphorylation (Garnerone et al., 1999). Other inhibitory proteins are KipI and Sda from B. subtilis. Sda prevents sporulation by inhibiting the primary HK proteins of the sporulation phosphorelay, KinA and KinB (Burkholder et al., 2001). Like Sda, KipI is an inhibitor of the autophosphorylation of KinA, targeting the C-terminal transmitter domain of the protein. It is thought that KipI-KipA interaction, in response to an additional so far unknown stimulus, regulates the anti-kinase activity of KipI (Wang et al., 1997).

In the following paragraph, some membrane anchored accessory proteins reminescent of LiaF will be presented with focus on those accessory proteins associated with intramembranesensing HK (UhpB, KinB) or those TCS involved in responding to CES (Cpx, Cse). Their role for signal perception is divers. Generally, deletion of their genes results in the loss of 
functional TCS systems. The proteins can be either specific components of the TCS, or required for stimulus perception and kinase activity, potentially by serving as ligand-binding proteins, such as UhpC and $\mathrm{KbaA} / \mathrm{KapB}$.

Table 4.1. Accessory proteins for TCS-mediated stimulus perception

\begin{tabular}{|c|c|c|c|c|}
\hline \multirow{2}{*}{$\begin{array}{l}\text { Sensor type/ } \\
\text { name (org. }{ }^{1} \text { ) }\end{array}$} & \multicolumn{2}{|c|}{ accessory protein } & \multirow[t]{2}{*}{ stimulus $^{2}$} & \multirow[t]{2}{*}{ references } \\
\hline & $\overline{\text { name }}$ & localization & & \\
\hline \multicolumn{5}{|c|}{ periplasmic-sensing } \\
\hline VirA (Atu) & ChvE & periplasmic & monosacch. & (Shimoda et al., 1993) \\
\hline BctE (Bpe) & $\mathrm{BctC}$ & periplasmic & citrate & (Antoine et al., 2005) \\
\hline LuxQ (Vha) & LuxP & periplasmic & AHL & (Bassler et al., 1994) \\
\hline TorS (Eco) & TorC & membrane & TMAO & (Gon et al., 2001) \\
\hline YycG (Bsu) & YycH & membrane & $?$ & (Szurmant et al., 2005) \\
\hline \multicolumn{5}{|c|}{ intramembrane-sensing } \\
\hline LiaS (Bsu) & $\mathrm{LiaF}$ & membrane & env. stress & (Jordan et al., 2006; Mascher, 2006a) \\
\hline UhpB (Eco) & UhpC & membrane & Glu6P & (Kadner, 1995) \\
\hline KinB (Bsu) & $\mathrm{KbaA} / \mathrm{KapB}$ & membrane & $\mathrm{C} 1$ pool? & (Dartois et al., 1996; Dartois et al., 1997a; Dartois et al., 1997b) \\
\hline RegB, PrrB & SenC, $\operatorname{PrrC}^{3}$ & membrane & redox status & (Eraso and Kaplan, 2000; Swem et al., 2005) \\
\hline \multicolumn{5}{|c|}{ cytoplasmic-sensing } \\
\hline [CheA (var.) & MCP's] & membrane & var. solutes & (Bass and Falke, 1999; Bilwes et al., 2003; Grebe and Stock, 1998) \\
\hline NreB (Sca) & NreA? & membrane & nitrate? & (Fedtke et al., 2002) \\
\hline HoxJ (Reu) & HoxBC & soluble & $\mathrm{H}_{2}$ & (Buhrke et al., 2004; Friedrich et al., 2005) \\
\hline FixL (Sme) & FixT & soluble & low $\mathrm{O}_{2}$ & (Garnerone et al., 1999) \\
\hline KinA (Bsu) & KipI/Sda & soluble & energy status & (Wang et al., 1997) / (Burkholder et al., 2001) \\
\hline
\end{tabular}

${ }^{1}$ Organisms: var. (various), Atu (Agrobacterium tumefaciens), Bpe (Bordetella pertussis), Vha (Vibrio harveyi), Bsu (Bacillus subtilis), Eco (Escherichia coli), Reu (Ralstonia eutropha), Sme (Sinorhizobium meliloti), Sca (Staphylococcus carnosus). ${ }^{2}$ Stimuli: monosacch. (monosaccharides), AHL (acyl homoserine lactone), TMAO (trimethyl amine-N-oxide), env. stress (cell envelope stress), $\mathrm{C} 1$ pool (cellular pool of one-carbon units), Glu6P (glucose-6-phosphate), var. solutes (amino acids, monosaccharides, peptides, metal ions). ${ }^{3}$ RegB-SenC/PrrBPrrC homologs are found in purple nonsulfur photosynthetic bacteria.

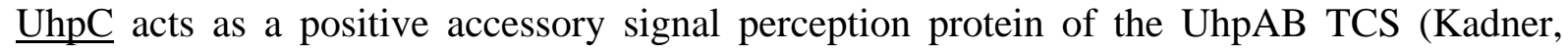
1995). This system is induced by the presence of extracellular glucose-6-phosphate and regulates the expression of the sugar-phosphate transport protein UhpT (Weston and Kadner, 1988). UhpC is a transmembrane protein with 12 TMRs and shares a high sequence similarity with the transport protein UhpT. Deletion of $u h p C$ results in a complete loss of Glu6Pdependent induction of $u h p T$ expression. Therefore, both UhpC and the intramembranesensing HK UhpB (8 TMRs) are required for the Glu6P-inducible auto-phosphorylation activity. Mutational analyses indicated that the interaction between UhpC and UhpB occurs within the membrane interface between the hydrophobic membrane-spanning C-termini of both proteins (Island and Kadner, 1993). 
Kba/KapB: One of the most complex known phosphorelay systems regulates endospore formation in the Gram-positive soil bacterium B. subtilis (Errington, 2003; Msadek, 1999; Piggot and Hilbert, 2004). Sensory information from five different kinases are integrated through the phosphorylation of the key sporulation transcription factor Spo0A. Its activation by phosphotransfer from the sensor kinases via the primary RR SpoOF and a phosphotransferase, Spo0B, triggers the commited step of sporulation (Errington, 2003). The two major suppliers of phosphate into the phosphorelay are the soluble, cytoplasmic-sensing HK KinA (which was shown to be influenced by soluble accessory proteins, Table 4.1) and the membrane-bound KinB. KinB contains six potential TM regions without significant periplasmic linkers (Trach and Hoch, 1993), suggesting that it does not directly sense extracellular effector molecules. KinB also contains no cytoplasmic linker or sensory domains and no conserved sequence features can be detected in the input domain. Rather, the mechanism of signal perception seems to be mediated by two membrane-anchored accessory stimulus perception proteins that are involved in activating the intramembrane-sensing $\mathrm{HK}$ KinB of B. subtilis: KbaA, a TM protein with six putative TM helices, and KapB. The product of $k a p B$, a gene co-transcribed in an operon with and located directly downstream of $k i n B$, is a lipoprotein that is tethered to the outer face of the cytoplasmic membrane after lipidmodification of cysteine residues (Dartois et al., 1996; Dartois et al., 1997a). Inactivation of $k a p B$ leads to a loss of KinB activity and confers the same phenotype as does a null mutation in kinB (Trach and Hoch, 1993). While a direct interaction of KapB and KinB has not been demonstrated, it was shown that the effect of a KapB inactivation is mediated through the Nterminal, membrane-spanning input-domain of KinB. KapB was discussed as a potential ligand-binding protein that interacts with the 6TMR-input domain of KinB, a situation somehow reminiscent of the UhpBC system (Dartois et al., 1997a). The nature of these protein-protein interactions and the signals sensed through KbaA and KapB are still unknown. But it was demonstrated that depletion of intracellular one-carbon units inactivates the KinBdependent signal transduction pathway (Dartois et al., 1997b).

CpxP: The CpxAR TCS is activated by elevated external $\mathrm{pH}$, misfolded periplasmic proteins or changes in the lipid composition of the inner membrane (Ruiz and Silhavy, 2005). It is subject to a negative feedback regulation exerted by the periplasmic protein CpxP. The Nterminal $\alpha$-helix of CpxP plays an important role for both, inhibition of CpxA via its sensing domain, as well as stabilization of CpxP (Buelow and Raivio, 2005). Direct protein-protein interaction between the sensor kinase $\mathrm{CpxA}$ and the periplasmic protein $\mathrm{CpxP}$ results in a down-regulation of the autokinase activity of CpxA (Fleischer et al., 2007), while DegP- 
mediated proteolysis of $\mathrm{CpxP}$ relieves inhibition of the Cpx response in the presence of inducing cues (Buelow and Raivio, 2005).

YycI/YycH: YycFG of B. subtilis was the first Gram-positive TCS to be described as essential for survival under normal laboratory conditions (Fabret and Hoch, 1998). It is restricted to, and conserved in the Firmicutes bacteria. Its essentiality has been verified in number of these bacteria (Clausen et al., 2003; Hancock and Perego, 2004; Lange et al., 1999; Williams et al., 2005), with the noteworthy exception of Lactococcus lactis (O'ConnellMotherway et al., 2000). Two major groups of YycFG-like TCS can be distinguished. Group I, which is found in most Firmicutes bacteria, is characterized by an extended genomic context conservation, with three to four genes, including $y y c H$ and $y y c I$ and its homologs, located directly downstream of the $y y c F G$ operon. The N-terminus of the YycG HK consists of two deduced transmembrane helices that flank a large periplasmic domain. In this group, both HK and RR are essential. The YycFG system of B. subtilis seems to be (at least partially) active during normal growth, and no inducing conditions have been identified so far. Its activity is regulated by $\mathrm{YycI}$ and $\mathrm{YycH}$ through direct protein-protein interactions with the sensor kinase YycG, (negatively) affecting its autophosphorylation activity (Szurmant et al., 2005; Szurmant et al., 2007a). Both proteins are peripherally bound to the cytoplasmic membrane and harbor large periplasmic domains that are similar in fold, but not in primary amino acid sequence (Santelli et al., 2007; Szurmant et al., 2006). Surprisingly, the transmembrane helix of each protein is sufficient to perform the regulatory role described for YycH and YycI (Szurmant et al., 2007b).

CseA: The $\sigma^{\mathrm{E}}$-CseABC pathway is unique in incorporating an ECF sigma factor, a TCS, and a novel accessory lipoprotein into a single signal transduction pathway (Hutchings et al., 2006a; Paget et al., 1999a; Paget et al., 1999b). It is also unusual amongst envelope stress ECF $\sigma$ factors in that it is not under the control of an anti- $\sigma$ factor. This system is conserved in all streptomycetes genomes sequenced to date and appears to be the major pathway for sensing cell envelope stress in the genus Streptomyces. Expression of the sigE-cseABC operon is regulated by a novel three-component system consisting of a sensor kinase, CseC, a RR, $\mathrm{CseB}$, and an accessory lipoprotein, CseA. CseC is proposed to bind a cell wall precursor or breakdown product since expression of the sigE operon can be induced by a wide range of cell envelope-specific compounds, including antibiotics such as bacitracin or vancomycin, and muramidases such as lysozyme (Hong et al., 2002). Disruption of the cseA gene led to a five-fold increase in this basal activity, suggesting that CseA negatively regulates the sigE promoter. However, since it is an extracytoplasmic lipoprotein, it must do so from the outside 
of the cell and it seems likely that CseA negatively modulates the sensor domain of CseC (Hutchings et al., 2006a). CseA has no homologs outside of the streptomycetes and, so far, there are no clues as to how it might modulate signal sensing by CseC. Transcription of sigE is still inducible by cell envelope specific antibiotics in a $\Delta c s e A$ strain and is induced to a higher level than in the wild-type (Hutchings et al., 2006a). This suggests that CseA somehow reduces the activity of $\mathrm{CseC}$, perhaps by reducing signal binding or trapping it in the 'OFF' state. A closely CseC-related HK is MtrB. As well as CseC, MtrB is encoded in an operon together with its RR and a lipoprotein, LpqB. Like CseA, LpqB mediates signal transduction through MtrAB by interacting with the sensor domain of MtrB (Hoskisson and Hutchings, 2006).

As described above, accessory proteins have various functions and can form a sensory unit together with the HK (like ChvE) or repress the activity of HK in different ways (Sda, KipI, TorC). It was shown that LiaF represses the activation of LiaS, but the mechanism is unknown. Maybe LiaF forms a sensory unit together with LiaS, releasing LiaS in its active form after sensing a signal. The second possibility is that LiaF represses the kinase activity of LiaS in a different way. Initial results on the role of the LiaF/LiaS sensory unit will be described in the next paragraph.

\section{Stimulus perception of the LiaFSR three-component system}

During this work it could be demonstrated that LiaF and LiaS are regulators of LiaR activity. Under conditions of cell envelope stress LiaS activates the RR by transferring a phosphate to the $\mathrm{Asp}_{54}$ of LiaR, while LiaF represses this activation in the absence of cell envelope stress. The mechanism of this repression remains unclear and its identification will be part of further studies. It seems likely that LiaF interacts directly with LiaS, because both proteins are membrane anchored and therefore perhaps co-localized, something that needs to be verified experimentally. But two questions still remain unanswered: What is the signal that is sensed by the lia system and how is this sensing accomplished? The first approach to address the second question was to identify amino acids in LiaF and LiaS that are essiential for their function.

Mutagenesis of LiaF and LiaS. Initially, truncated alleles of both liaS and liaF were constructed. Moreover, conserved residues in both genes were replaced by alanine. The clean deletion mutants were then complemented with the mutated versions of LiaF and LiaS to identify amino acids and regions within the two proteins that are necessary for their function. 
Such an experimental set-up was successfully used to identify essential regions in other histidine kinases from B. subtilis, such as ResE and PhoR (Baruah et al., 2004; Eldakak and Hulett, 2007).
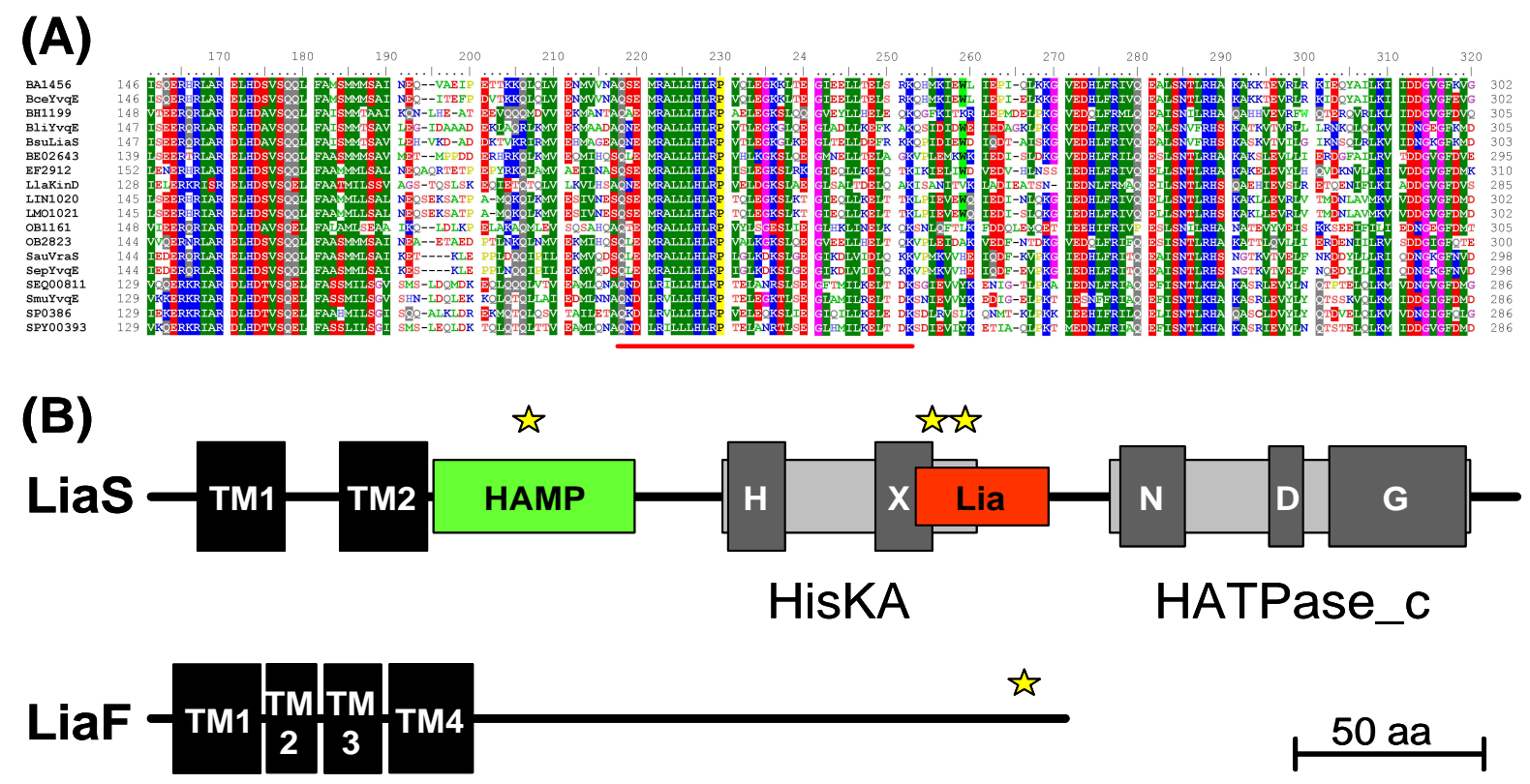

(C)

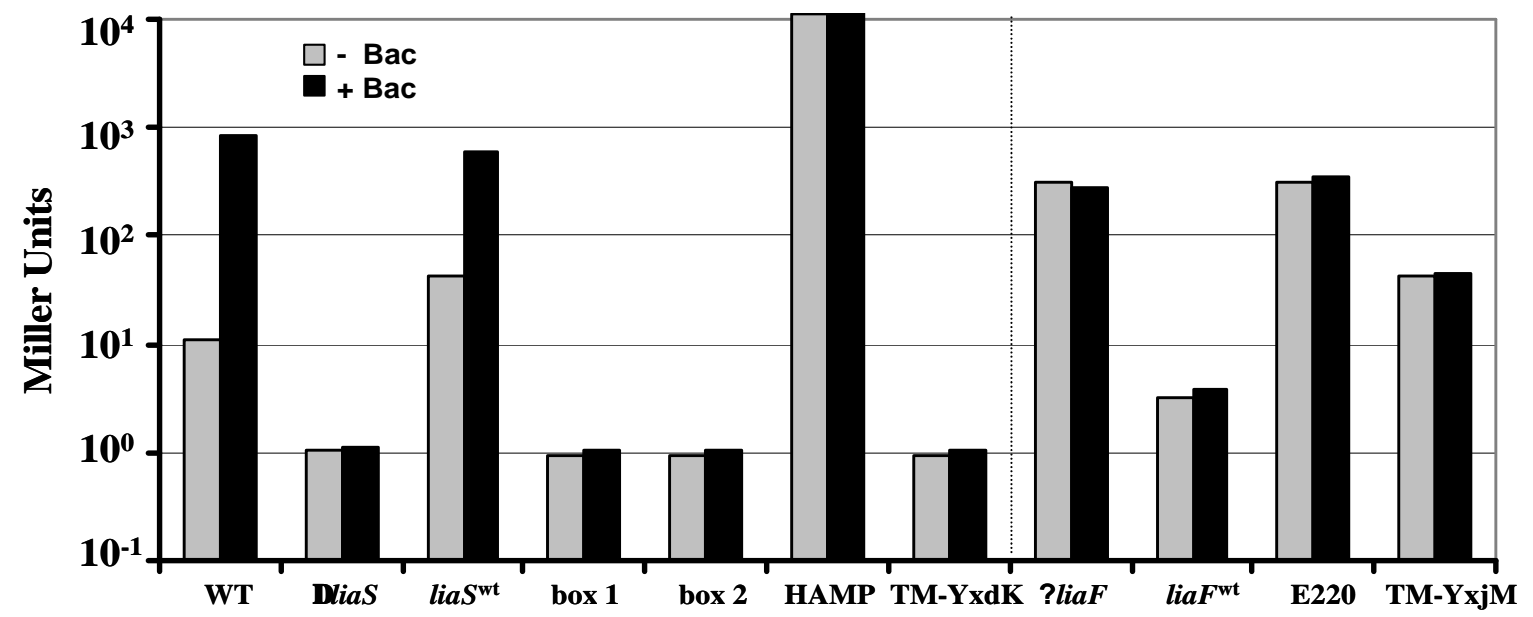

Figure 4.3. Mutagenesis of LiaS and LiaF. (A) Alignment of LiaS homologous kinases. The LiaS-box is underlined red. (B) Schematic presentation of LiaS and LiaF. The HAMP domain of LiaS is illustrated in green, the LiaS-box in red. The other boxes in the HisKA and HATPase_c region (light grey) are illustrated in dark grey. Location of mutations are indicated with stars. (C) Results of the $\beta$-galactosidase assay. WT $=$ TMB370, lia $S^{\mathrm{wt}} / l i a F^{\mathrm{wt}}$ represent complementation with wildtype copy of the deleted gene. Box 1 is aa $\mathrm{Q}_{202}-\mathrm{A}_{207}$, box 2 $\mathrm{H}_{211}-\mathrm{P}_{214}$. TM stands for replacement of the transmembrane domains by that of the following protein. E220 = protein ends after amino acid $\mathrm{E}_{220}$. See text for details

All LiaS homologs contain a highly conserved motif, which is not present in any other protein. This motif is located between the $\mathrm{X}$-box and the N-box in the cytoplasmic transmitter domain of LiaS (Fig. 4.3A). Since it serves as a signature sequence for this group of kinases, 
we termed it the "LiaS-box". Preliminary results revealed that the LiaS-box is essential for the function of LiaS. Both, deletion and alanine replacement mutagenesis of parts of the Lia-box (motives $\mathrm{Q}_{202}-\mathrm{A}_{207}$ and $\mathrm{H}_{211}-\mathrm{P}_{214}$ ) lead to a non-inducible kinase (Fig. 4.3B and C). In-depth characterization of the LiaS-box will be subject to future studies.

The HAMP ( $\underline{h}$ istidine kinase, $\underline{a}$ denylyl cyclase, $\underline{m}$ ethyl-accepting chemotaxis protein and phosphatase) domain is a region of approximately 50 residues located after the transmembrane domain 2 in LiaS. It consists of two amphipathic helices with coiled-coil properties (Butler and Falke, 1998) and links the input domain to the cytoplasmic transmitter domain. The HAMP domain is thought to transduce the incoming signal by rotation of its helices (Hulko et al., 2006). As shown for the nitrate sensor NarX from E. coli, changing of the highly conserved glutamate within the HAMP linker results in a constitutively active kinase, due to structural changes (Appleman and Stewart, 2003). The same behaviour could be observed for LiaS: Deletion or alanine replacement of the amino acids DDE on position 104-106 lead to a constitutively active kinase, resulting in the permanent induction of $\mathrm{P}_{\text {lial }}$ (Fig. 4.3C). Interestingly, LiaF is not able to repress LiaS activity in this mutant.

Because of the lack of an extracytoplasmic loop, which is thought to function as the sensory domain in other families of histidine kinases, LiaS belongs to the so-called intramembranesensing HK (IMHK) (Mascher, 2006b), which are thought to sense their inducing signal directly within the membrane (Mascher et al., 2006). To confirm this hypothesis, it was important to check whether the transmembrane domains of LiaS are necessary for stimulus perception or if only the location at the membrane (and/or the contact to LiaF) is sufficient for the function of LiaS. Therefore, a hybrid kinase was constructed by replacing the transmembrane domains of LiaS with the 2 transmembrane domains of YxdK. YxdK is also an IMHK, which is not induced by bacitracin. If membrane-anchoring alone would be sufficient for LiaS function, it should be possible to activate this hybrid kinase with bacitracin. Instead, this YxdK-LiaS hybrid is not induceable by bacitracin (Fig. 4.3C). The same behaviour was observed after deletion of the TM (data not shown). Therefore, the specific N-terminal domain of LiaS is important for stimulus perception.

In contrast, a hybrid allele of LiaF with the 4 transmembrane domains of YxjM is able to complement the liaF deletion, at least to some extend ( 10-fold reduction) (Fig. 4.3C). In fact, preliminary data indicate that even a soluble C-terminal fragment of LiaF, lacking all four TMR, is sufficient for partially complementing a liaF deletion (data not shown). These findings indicate that the C-terminal part of $\mathrm{LiaF}$ interacts with $\mathrm{LiaS}$ and inhibits autophosphorylation. This assumption is supported by the finding that even the last 25 amino 
acids of LiaF are essential for its function. Their deletion (position 220 to 245) leads to a complete loss of LiaF function. This mutant behaves exactly like the liaF deletion mutant (Fig. 4.3C). Future studies will hopefully reveal which of the 25 amino acids in this region are involved in the repressory function of LiaF. Good candidates are two conserved aspartates at positions 235 and 237. So far, alanine scanning mutagenesis of any other conserved amino acid in the C-terminus did not reveal any crucial residue (data not shown).

While it could be demonstrated that the TM of LiaS are essential for stimulus perception, the exact nature of the inducing signal remains unclear. A direct interaction of the known inducers with LiaS seems unlikely because of their diversity. It has been postulated that the stimulus perception via the transmembrane helices occurs directly at the membrane interface (Mascher et al., 2003; Mascher et al., 2006).

Additionally, the mechanism of LiaF function needs to be investigated further. The data so far indicates that LiaF has both sensory and repressory function.

\section{Interaction of the ECF $\sigma$ factor $\sigma^{\mathrm{W}}$ with the LiaFSR three-component system}

The ECF sigma factor $\sigma^{\mathrm{W}}$, which is one of three ECF sigma factors involved in the cell envelope stress response in B. subtilis (Mascher et al., 2003), was shown to positively affect the expression of the lia system under inducing conditions. Overexpression of $\sigma^{\mathrm{W}}$ results in a 16-fold increased amount of lialH transcript (Asai et al., 2003). Moreover, there is a significant overlap of the inducer spectrum of both systems. $\sigma^{\mathrm{W}}$ is strongly induced by alkaline shock (Wiegert et al., 2001), vancomycin, cephalosporine, D-cycloserine, Triton X100 (Cao et al., 2002a) and CAMPs (Pietiäinen et al., 2005). Weak inducers are bacitracin, fosfomycin and tunicamycin (Cao et al., 2002a). The response of the lia system to alkaline shock is very weak and was demonstrated to be $\sigma^{\mathrm{W}}$-dependent (Wiegert et al., 2001): in a sigW mutant, no alkaline shock induction of liaIH was observed any longer. A somewhat similar behaviour was also observed for CAMP induction (Pietiäinen et al., 2005). These results suggest that $\sigma^{\mathrm{W}}$ itself, or $\sigma^{\mathrm{W}}$-upregulated genes induce the LiaFSR three-component system under certain conditions (Fig. 4.4). Since a direct binding of $\sigma^{\mathrm{W}}$ to the liaI promoter can be ruled out, the positive effect of $\sigma^{\mathrm{W}}$ excerted on $\mathrm{P}_{\text {lial }}$-dependent expression seems to be indirect. One possible explanation could be that the induction of members of the $\sigma^{\mathrm{W}}$ regulon themself provide the stimulus for LiaRS-dependent gene expression. Given the role of $\sigma^{\mathrm{W}}$ in counteracting and expressing antimicrobial compounds (Butcher and Helmann, 2006; Helmann, 2002, 2006), this seems to be an alternative hypothesis. A systematic screen of the 
inducing potential of the $\sigma^{\mathrm{W}}$ regulon on liaIH expression is currently under way in collaboration with John Helmann.

Interestingly, $\sigma^{\mathrm{W}}$ expression starts in post-exponential growth phase in a culture of $B$. subtilis cells (Huang et al., 1998), similar to the LiaR-dependent expression of LiaI and LiaH. $\sigma^{\mathrm{W}}$ expression and the expression of its target genes is regulated by Spo0A and AbrB (Qian et al., 2002), another commonality to the lia system. In this case, Spo0A positively affects induction of sigW expression by repressing $a b r B$ expression. The same mechanism could be demonstrated for the regulation of $\mathrm{P}_{\text {lial }}$ induction as well (chapter 3).

In addition to the regulatory function of Spo0A on transition state induction, the presence of high amounts of phosphorylated Spo0A was shown to positively affect the expression of liaIH (Fujita et al., 2005).

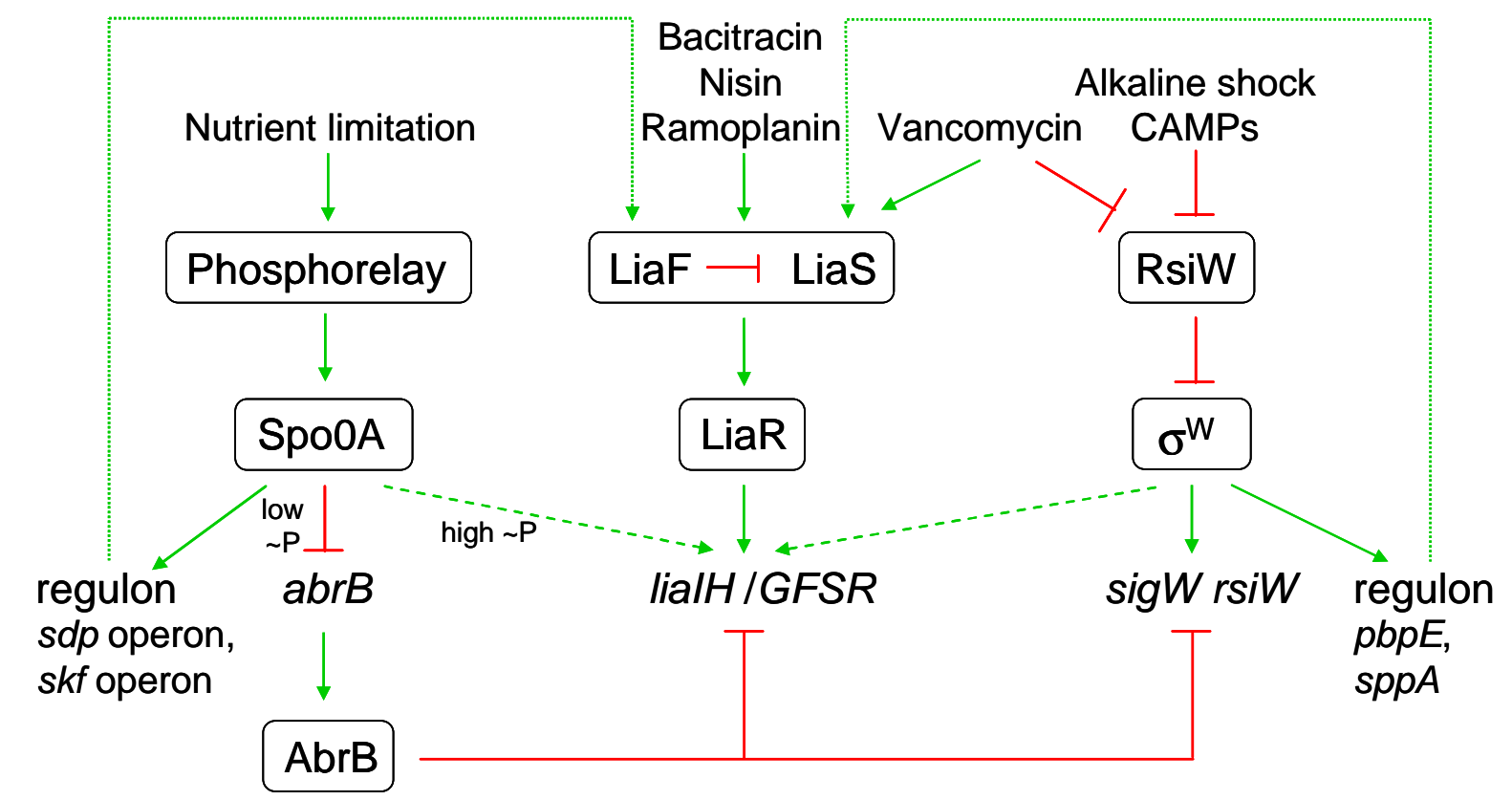

Figure 4.4. Model of LiaFSR regulation. Arrows indicate induction, T-shaped lines repression. Stripe lines indicate indirect regulations, dottet lines postulated stimulatory interactions. See text for details.

It still remains unclear what kind of signal is sensed by LiaFS. Lipid II-interacting antibiotics, secretion stress and entry of stationary phase all induce $\mathrm{P}_{\text {lial }}$. One similarity of all these inducing conditions is that they affect some aspect of the flow of metabolites through the membrane. Lipid II-interacting antibiotics change the amount of lipid II that flips in and out. In stationary phase cell wall synthesis decreases, ultimately affecting the lipid II cycle in a way compareable to the antibiotics. Under conditions of secretion stress, more proteins than normal are transferred through the membrane. Therefore, we speculate that the Lia system senses "membrane trafficing" and not envelope perturbation per se. 


\section{Stoichiometry of LiaFSR proteins}

The LiaFSR three-component system tightly regulates the expression of the lia operon. Deletion and complementation studies revealed that mutations or insertions within the lia operon, which lead to different expression levels of the proteins LiaF, LiaS or LiaR, result in significant changes of the behaviour of the LiaFSR three-component system. Overexpression of LiaR results in an induction of $\mathrm{P}_{\text {lial }}$ (Kobayashi et al., 2001), even in the absence of LiaS (Jordan et al., 2006). Overexpression of LiaS lead to the same behaviour (see below). $\mathrm{P}_{\text {lial }}$ induction is lost in a liaR mutant (Jordan et al., 2006) and in a clean liaS mutant (see below). Deletion of LiaF results in a constitutive active LiaRS TCS (Jordan et al., 2006).

The genes encoding the LiaFSR three-component system are cotranscribed, but analysis of their Shine-Dalgarno (SD) sequences indicated that they are translated with different efficiency, resulting in different amounts of the three proteins in the cell. Conservation of the SD sequence and its distance to the start codon are the determining factors for the translation efficiency (Vellanoweth and Rabinowitz, 1992). liaF has a perfect SD (Fig. 4.5) with almost the optimal spacing (12 bp) upstream of its start codon. This might lead to high amounts of LiaF in the cell. liaR has also a strong SD with 8 bp spacing. The SD of liaS is poorly conserved, only 4 of 7 nucleotides correspond to the optimal SD. Presumably, this leads to lower amounts of LiaS compared to LiaF and LiaR in the cell, so that the amount of LiaS is the limiting factor in LiaFSR three-component system. This assumption is supported by preliminary data from translational lacZ fusions, showing a 6-fold lower activity for liaS compared to liaF (Rietkötter, personal communication).

$\begin{array}{lcc} & \text { sequence } & \text { spacing } \\ \text { consensus } & \text { AAGGAGG } & \mathbf{7 - 9} \\ \text { liaF } & \text { AAGGAGG } & 12 \\ \text { liaR } & \text { AAGGAGA } & \mathbf{8} \\ \text { liaS } & \text { GTGGATG } & 10 \\ \text { liaS SD } & \text { AAGGAGG } & \mathbf{7}\end{array}$

Figure 4.5. Comparison of the Shine-Dalgarno sequences. Nucleotides corresponding to the consensus sequence are shaded in grey. Optimal spacing of the SD to the start codon is written in bold. liaS SD is the mutated allele with the optimal Shine-Dalgarno sequence and spacing. See text for details.

Changes in the amount of one of the three proteins LiaF, LiaS and LiaR strongly affect the function of the system. The most striking effect was observed during complementation studies of liaF mutants (Fig. 4.6). In a liaF::kan mutant, $\mathrm{P}_{\text {lial }}$ is induced to $~ 3000$ Miller Units (Jordan et al., 2006), i.e. a 30-fold increased induction compared to the bacitracin-induced 
wildtype. Subsequent analysis indicated that this behaviour was due to the positive polar effect of the kanamycin resistance cassette, which was introduced in this strain upstream of the liaSR genes. A non-polar, i. e. clean liaF deletion ( $\Delta$ liaF) shows $\mathrm{P}_{\text {lial }}$ induction comparable to the level of the induced wildtype ( 100 Miller Units, see Fig. 4.6). In this strain the TCS is fully active. The liaF::kan strain lacks the repressor LiaF and additionally strongly overproduces LiaS and LiaR. Obviously, these two factors together lead to the increased $\mathrm{P}_{\text {lial }}$ activity in the liaF::kan mutant. When a wildtype copy of liaF is introduced into this strain ectopically under control of a xylose-inducible promoter (using pXT), $\mathrm{P}_{\text {lial }}$ induction is reduced 10-fold to $\sim 100$ Miller Units (Fig. 4.5). This complementation is therefore only partial and the system no longer responds to bacitracin, maybe as a result of insufficient amounts of LiaF compared to LiaRS. A different behaviour is detectable in the liaF clean deletion mutant. With an ectopical wildtype copy of liaF, the $\mathrm{P}_{\text {lial }}$ activity is reduced nearly to the level of the uninduced wildtype. But again the system does not respond to the presence of bacitracin. The reason for the lack of induction is unclear at the moment.

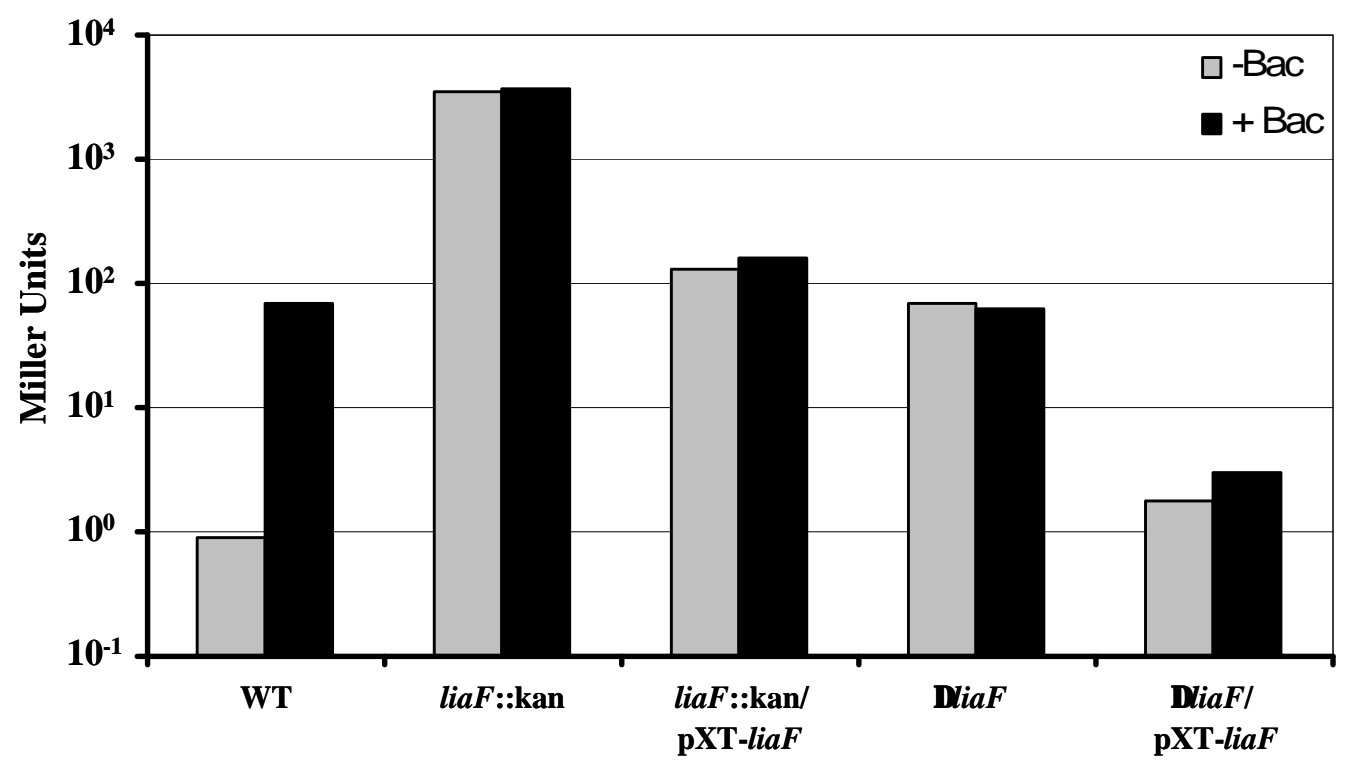

Figure 4.6. Complementation of liaF mutants. liaF::kan means replacement of liaF by a kanamycin resistance cassette. $\Delta l i a F$ is the clean deletion mutant. The reporter strain is based on pAC6 (TMB016). See text for details.

As shown in chapter 2, the replacement of liaS by a kanamycin resistance cassette results in a significant background activity of the liaI promoter. Again we could demonstrate that this was due to the positive polar effect of the resistance cassette. Quantitative real-time RT-PCR revealed that there is a 34-fold increased level of the liaR mRNA in the liaS::kan mutant compared to the wildtype. Under these conditions, LiaR becomes unspecifically 
phosphorylated and activates $\mathrm{P}_{\text {lial }}$, even in the absence of LiaS. Changing the Asp $\mathrm{As}_{54} \rightarrow \mathrm{Ala}-$ the residue to which the phosphate is transferred from the histidine kinase LiaS - abolishes $\mathrm{P}_{\text {lial }}$ activity despite strongly increased LiaR amounts in the cell (Fig. 4.7). This experiment demonstrated that overexpression of LiaR in the cell alone is not sufficient for $\mathrm{P}_{\text {lial }}$ activation and that an unspecific phosphate donor for LiaR is required in the absence of LiaS.

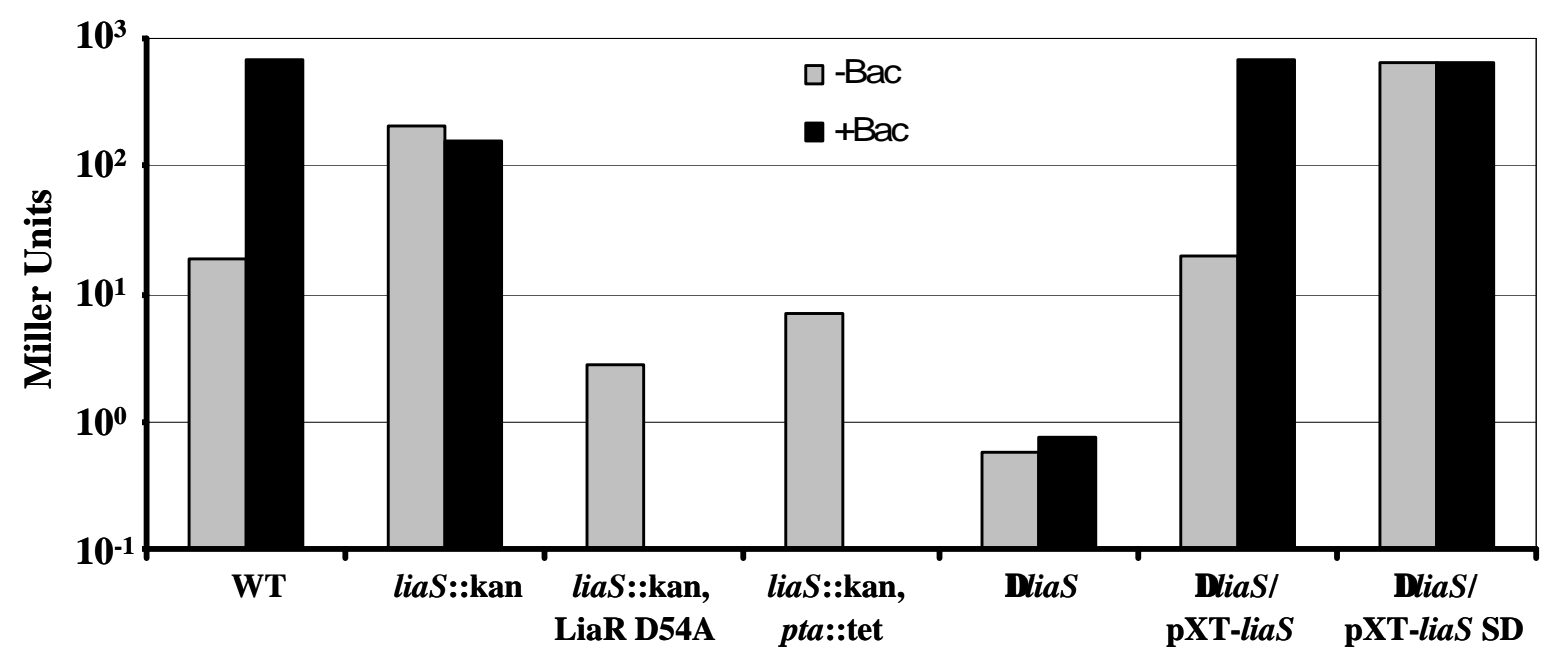

Figure 4.7. Phosphorylation of LiaR in different mutants. liaS::kan means replacement of liaS by a kanamycin resistance cassette. tet is the tetracyclin resistance cassette. $\Delta$ liaS is the clean deletion mutant. SD is the optimal Shine-Dalgarno sequence. For the liaS::kan, LiaR D54A and the liaS::kan, pta::tet mutants $\beta$ galactosidase activity was only determined without bacitracin induction. The reporter strain is based on pMUTIN (TMB370). For details see text.

Acetyl phosphate is known to be able to function as a phosphodonor for RR. Recently it was demonstrated for E. coli, that the intracellular concentration of acetyl phosphate in this bacterium $(3 \mathrm{mM})$ is sufficient to activate RR via direct phosphoryl transfer (Klein et al., 2007). An example illustrating the physiological role of acetyl phosphate in $R R$ phosphorylation is VanR, the RR mediating vancomycin resistance in Streptomyces coelicolor. It recently was shown to become fully phosphorylated by acetyl phosphate in the absence of both the cognate HK and the external inducer, vancomycin (Hutchings et al., 2006b). Under non-inducing conditions, the cognate HK VanS acts as a phosphatase to prevent this unspecific phosphorylation.

We could demonstrate that in the liaS::kan mutant acetyl phosphate serves as the phospodonor for LiaR in vivo. In a liaS::kan, pta::tet mutant, which should not be able to synthesize acetyl phosphate, the activation of LiaR is gone (Fig. 4.7). But one should bear in mind that the phosphorylation of LiaR by acetyl phosphate is artificial and occurs only in the liaS::kan mutant, because of the high amounts of LiaR together with the loss of LiaS and its 
proposed phosphatase activity. Both, in the wildtype and a non-polar, i. e. clean liaS deletion mutant (TMB215), no increased basal activity of $\mathrm{P}_{\text {lial }}$ could be observed.

This clean liaS deletion mutant was complemented by introduction of a liaS wildtype copy (Fig. 4.7). When overexpressing LiaS with an optimized SD, the proportion of LiaS to the repressor LiaF is increased. Consequently, $\mathrm{P}_{\text {liaI }}$ is constitutively activated (Fig. 4.7). These findings support the hypothesis that LiaS is present in low amounts and is set to the phosphatase mode due to LiaF function.

Taken together, the findings so far indicate that the proteins LiaF, LiaS and LiaR have to be present in the cell in distinct amounts and that their ratios are crucial for the function of the LiaFSR three-component system. Any conditions affecting this balance result in the system being "blind" to cell envelope stress. Indeed, it is fixed to the "ON"- or "OFF"-state of the system, depending on the regulator reduced or overexpressed.

\section{Bistability of the liaI promoter}

As shown in chapter 3, the intrinsic transition state induction of $\mathrm{P}_{\text {lial }}$ is tightly regulated and embedded in the Spo0A/AbrB-dependent regulatory cascade. Interestingly, the maximum induction under those conditions is about 10-15-fold weaker than the induction with cell wall antibiotics. This observation can either be explained by an overall weaker promoter activity. Alternatively, it could be due to the full induction of $\mathrm{P}_{\text {liaI }}$ in only a subset of the culture, a phenomenon termed bistability.

To address this question for the transition state induction of $\mathrm{P}_{\text {lial }}$, a single cell approach is necessary to differentiate between induced and uninduced cells in the same culture. For that purpose, a $\mathrm{P}_{\text {lial }}$ - $g f p$ (green fluorescent protein) fusion was constructed and the resulting strain (TMB408) was monitored by fluorescence microscopy over several hours of growth at $30^{\circ} \mathrm{C}$. A strain carrying a $\mathrm{P}_{\text {liar }}$ lacZ fusion (TMB370) was grown in parallel and samples were taken for $\beta$-galactosidase assay at the same timepoints. The results from these initial experiments are promising. Only 10-15\% of the cells in a culture induce $\mathrm{P}_{\text {lial }}$ when entering stationary phase (Fig. 4.8). Monitoring this culture for two more hours in stationary phase (Fig. 4.8, 8 and $9 \mathrm{~h}$ ) clearly demonstrated that during the whole transition state only a subpopulation of cells induce $\mathrm{P}_{\text {lial }}$. This result explains the low induction level in transition state measured with the $\beta$-galactosidase assay and is a clear indication that $\mathrm{P}_{\text {lial }}$ is subject to a bistable switch. 

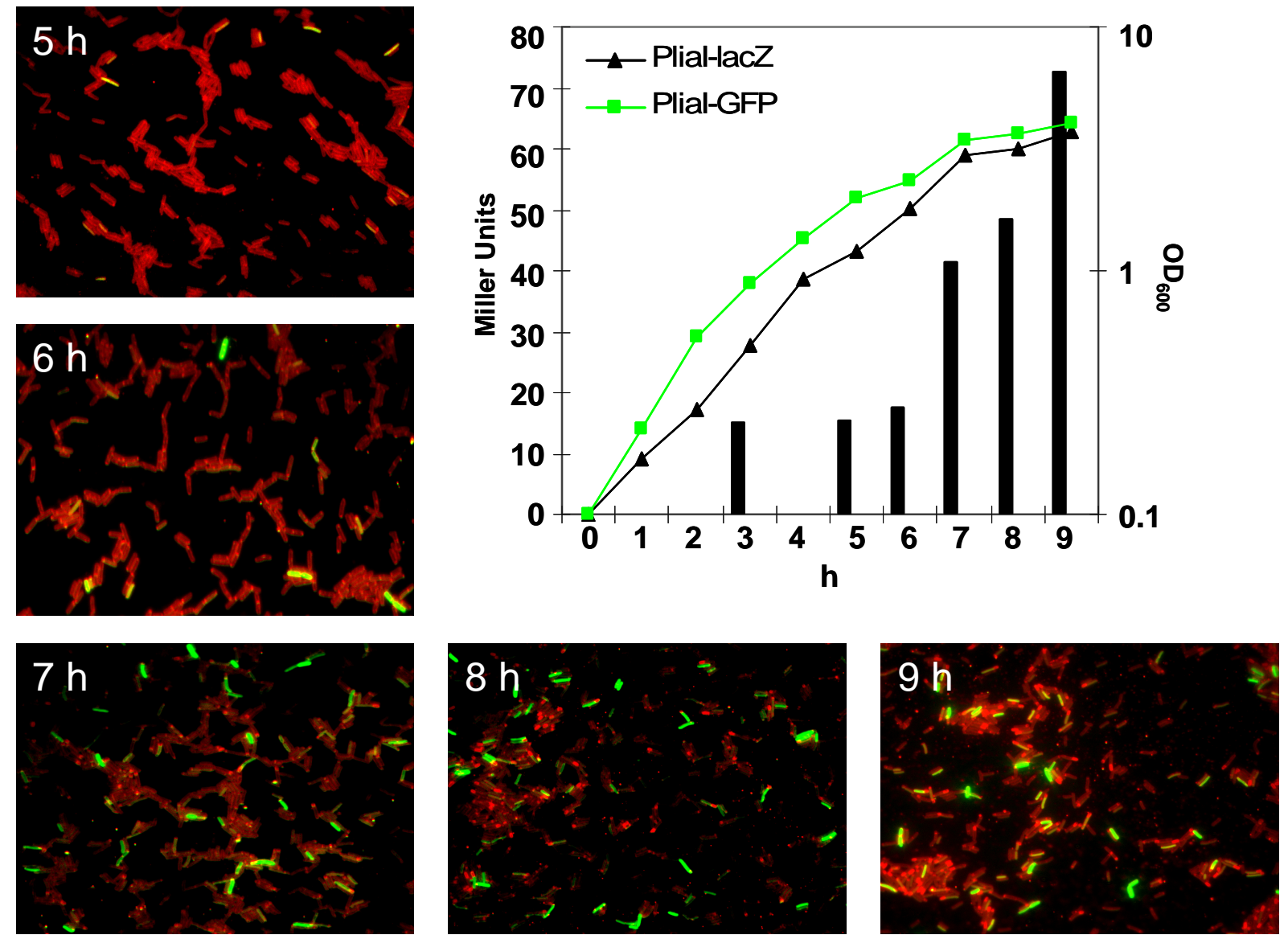

Figure 4.8. Bistable induction of $\mathbf{P}_{\text {lial }}$. GFP is expressed under the control of the liaI promoter. Samples were taken from TMB370 (black triangles) every hour for $\beta$-galactosidase assay (black bars). Cells from TMB408 (green squares) were monitored every hour by fluorescence microscopy. During transition state, only a subset of cells induce $\mathrm{P}_{\text {lial }}$ (fluorescence shown in green). The membrane of all cells was stained (red).

Bistability occurs in populations of isogenic cells, grown under identical conditions (e.g. in liquid media in well-stirred flasks). It requires that a regulatory system can only switch between two alternative states but cannot rest in between (Dubnau and Losick, 2006). The heterogeneous output of several bacterial processes that are subject to a bistable behaviour could be traced back to the feedback-based wiring of the network involved (Ozbudak et al., 2004; Smits et al., 2005; Veening et al., 2005). Two mechanisms have been proposed to drive bistable switches: positive or double-negative feedback regulation (Ferrell, 2002) (Fig. 4.9). The first mechanism (Fig. 4.9 left side) requires that the master regulator gene is positively autoregulated and responds to itself in a non-linear fashion (Becskei et al., 2001). Nonlinearity makes the response hypersensitive to even slight changes in regulator concentrations. Cells with regulator amount below the threshold do not switch on the output at all, while cells with more than the threshold amount of the regulator are driven to accumulate even more, due to the positive autoregulation. The expression of downstream genes is then altered and the population bifurcates. 
The second mechanism (Fig.4.9 right side) requires the presence of a pair of mutually repressing repressors (Gardner et al., 2000). If repressor 2 is inactivated then repressor 1 is produced, shutting off the synthesis of repressor 2. This step is equivalent to positive autoregulation, because the increase of repressor 1 results in its own production. If repressor 2 inhibits additional genes, these will now be expressed. If repressor 2 is not repressed, the system will behave the opposite way. One example of this kind of double-negative feedback regulation was observed in the switch between lysogenic and lytic states of the phage lambda (Ptashne, 2005).
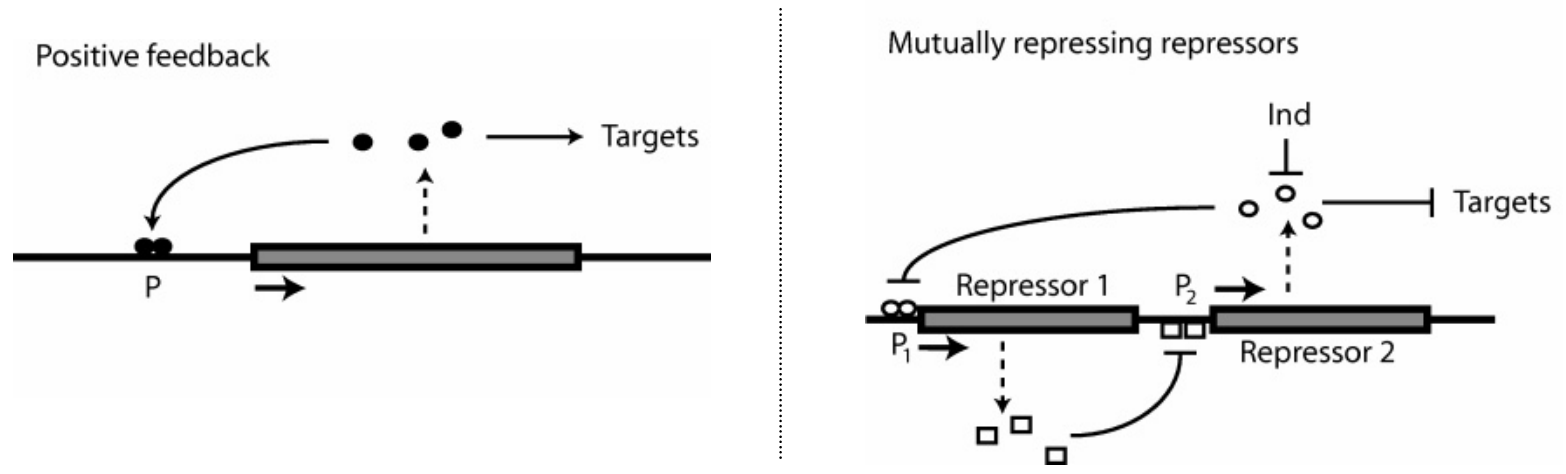

Figure 4.9. Two network configurations that lead to bistable expression. Figure taken from Dubnau and Losick, 2006. See text for details.

In B. subtilis several promoters are known to behave bistable (Dubnau and Losick, 2006). One example is the developement of competence. When cells enter stationary phase, about $10 \%$ of the cells in the culture become naturally competent because only in this subset of cells ComK activates the genes for DNA-uptake (van Sinderen et al., 1995). The developement of competence depends on positive feedback regulation. This regulation is hypersensitive to the concentration of ComK because the regulator binds to its own promoter $\mathrm{P}_{\text {comK }}$ as a dimer of dimers (Hamoen et al., 1998).

Swimming also shows a bistable behaviour (Kearns and Losick, 2005). Culures at the midexponential growth phase are a mixed population of cells in which $\sigma^{\mathrm{D}}$, the master regulator for swimming and cell separation, is active (swimming cells) and cells in which $\sigma^{\mathrm{D}}$ is inactive (long chains of non-motile cells).

Another well studied example for bistability is sporulation, regulated by the master regulator Spo0A. The activity of Spo0A is governed by phosphorylation via a multicomponent phosphorelay (Burbulys et al., 1991) and depends on nutrient limitation (Chung et al., 1994). It could be demonstrated for promoters under Spo0A control that they behave bistable as well (Gonzalez-Pastor et al., 2003). Since $\mathrm{P}_{\text {lial }}$ is indirectly controlled by Spo0A, too, it was not too 
suprising to find its transition state induction behave bistable. Furthermore, $\mathrm{P}_{\text {lial }}$ regulation contains all prerequisits for a bistable switch: A positive feedback loop while LiaR induces expression of the lia operon (Mascher et al., 2004) and an additionally regulation of LiaR activity, accomplished by LiaF and LiaS (Jordan et al., 2006). An in-depth analysis of the bistable switch governing $\mathrm{P}_{\text {lial }}$ activity will be subject of an upcoming $\mathrm{PhD}$ thesis. 


\section{Outlook}

The data presented in this thesis unraved the mechanism of signal transduction mediated by the cell envelope stress-sensing three-component system LiaFSR. The preliminary results described in the discussion represent the starting points for future investigations to address three primary questions: (1) What is the physiological role of the LiaRS TCS? (2) What is the nature of the stimulus sensed by this TCS? (3) What is the mechanism of stimulus perception mediated by the LiaF-LiaS sensory unit? (4) What is the mechanism behind the bistable behaviour of the liaI promoter?

LiaI and LiaH are the main targets of LiaR-dependent gene expression and should be studied in detail. Based on the similarity between LiaH and E. coli PspA, we propose that LiaI might function as the membrane anchor for LiaH. This assumption is supported by preliminary results indicating a co-localization of LiaH and LiaI. A phenotypical characterization of LiaH is currently under way.

Identifying the stimulus and unraveling the mechanism of stimulus perception by the LiaFLiaS sensory unit are two sides of a coin that need to be addressed together. Clearly, this represents a major goal of future research projects and will initially require the in-depth dissection of the two proteins involved. Based on the crucial role of both LiaF and LiaS for stimulus perception, we expect that they directly interact to form a sensory unit. This interaction could either occur within the membrane interface by the TMR of both proteins, or between the cytoplasmic domains, as has been suggested by the results from our initial mutagenesis studies. Mapping of the interacting interface between both proteins will therefore be an important prerequisite for understanding their function and hopefully also help to reveal the stimulus sensed by them.

The role of bistable switches in bacterial gene expression has only recently been recognized as a major mechanism to orchestrate population bifurcation at commiting regulatory checkpoints of differentiation cascades. With regard to the Lia system, the transition state induction of $\mathrm{P}_{\text {lial }}$ needs to be further analyzed by flow cytometry. Moreover, time-resolved quantitative data on protein stoichiometry (for LiaF, LiaS, and LiaR) and the phosphorylation state of LiaS and LiaF will allow mathematical modeling of the LiaFSR switch to ultimately explain the mechanism responsible for the bistable behaviour of $\mathrm{P}_{\text {lial }}$ induction. These analyses need to take into account the close link between the LiaRS TCS and the (phospho) neural network orchestrating the complex adaptional program that allows B. subtilis to adapt and ultimately survive under deteriorating growth conditions during stationary phase. 


\section{Chapter 5: References}

Adams, H., Teertstra, W., Demmers, J., Boesten, R., and Tommassen, J. (2003) Interactions between phage-shock proteins in Escherichia coli. J Bacteriol 185: 1174-1180.

Ades, S.E. (2004) Control of the alternative sigma factor $\sigma^{\mathrm{E}}$ in Escherichia coli. Curr Opin Microbiol 7: 157-162.

Alba, B.M., and Gross, C.A. (2004) Regulation of the Escherichia coli sigma-dependent envelope stress response. Mol Microbiol 52: 613-619.

Anantharaman, V., Koonin, E.V., and Aravind, L. (2001) Regulatory potential, phyletic distribution and evolution of ancient, intracellular small-molecule-binding domains. $J$ Mol Biol 307: 1271-1292.

Anke, T., (ed) (1997) Fungal Biotechnology. Weinheim: Chapman \& Hall.

Antoine, R., Huvent, I., Chemlal, K., Deray, I., Raze, D., Locht, C., and Jacob-Dubuisson, F. (2005) The periplasmic binding protein of a tripartite tricarboxylate transporter is involved in signal transduction. J Mol Biol 351: 799-809.

Appleman, J.A., and Stewart, V. (2003) Mutational analysis of a conserved signal-transducing element: the HAMP linker of the Escherichia coli nitrate sensor NarX. J. Bacteriol. 185: 89-97.

Archibald, A.R., Hancock, I.C., and Harwood, C.R. (1993) Cell wall structure, synthesis, and turnover. In Bacillus subtilis and Other Gram-Positive Bacteria. Sonenshein, A.L., Hoch, J.A. and Losick, R. (eds). Washington, D.C.: ASM press, pp. 381-410.

Arnaud, M., Chastanet, A., and Debarbouille, M. (2004) New vector for efficient allelic replacement in naturally nontransformable, low-GC-content, gram-positive bacteria. Appl Environ Microbiol 70: 6887-6891.

Asai, K., Yamaguchi, H., Kang, C.M., Yoshida, K., Fujita, Y., and Sadaie, Y. (2003) DNA microarray analysis of Bacillus subtilis sigma factors of extracytoplasmic function family. FEMS Microbiol Lett 220: 155-160.

Aseeva, E., Ossenbuhl, F., Eichacker, L.A., Wanner, G., Soll, J., and Vothknecht, U.C. (2004) Complex Formation of Vipp1 Depends on Its \{alpha\}-Helical PspA-like Domain. $J$. Biol. Chem. 279: 35535-35541.

Bailey, T.L., and Elkan, C. (1994) Fitting a mixture model by expectation maximization to discover motifs in biopolymers. Proc Int Conf Intell Syst Mol Biol 2: 28-36.

Bandow, J.E., Brotz, H., Leichert, L.I., Labischinski, H., and Hecker, M. (2003) Proteomic approach to understanding antibiotic action. Antimicrob Agents Chemother 47: 948955.

Baruah, A., Lindsey, B., Zhu, Y., and Nakano, M.M. (2004) Mutational Analysis of the Signal-Sensing Domain of ResE Histidine Kinase from Bacillus subtilis. J. Bacteriol. 186: $1694-1704$.

Bass, R.B., and Falke, J.J. (1999) The aspartate receptor cytoplasmic domain: in situ chemical analysis of structure, mechanism and dynamics. Structure Fold Des 7: 829-840.

Bassler, B.L., Wright, M., and Silverman, M.R. (1994) Multiple signalling systems controlling expression of luminescence in Vibrio harveyi: sequence and function of genes encoding a second sensory pathway. Mol Microbiol 13: 273-286.

Becskei, A., Seraphin, B., and Serrano, L. (2001) Positive feedback in eukaryotic gene networks: cell differentiation by graded to binary response conversion. Embo $J \mathbf{2 0}$ : 2528-2535.

Bentley, S.D., Chater, K.F., Cerdeno-Tarraga, A.M., Challis, G.L., Thomson, N.R., James, K.D., Harris, D.E., Quail, M.A., Kieser, H., Harper, D., Bateman, A., Brown, S., Chandra, G., Chen, C.W., Collins, M., Cronin, A., Fraser, A., Goble, A., Hidalgo, J., Hornsby, T., Howarth, S., Huang, C.H., Kieser, T., Larke, L., Murphy, L., Oliver, K., O'Neil, S., Rabbinowitsch, E., Rajandream, M.A., Rutherford, K., Rutter, S., Seeger, 
K., Saunders, D., Sharp, S., Squares, R., Squares, S., Taylor, K., Warren, T., Wietzorrek, A., Woodward, J., Barrell, B.G., Parkhill, J., and Hopwood, D.A. (2002) Complete genome sequence of the model actinomycete Streptomyces coelicolor A3(2). Nature 417: 141-147.

Berdy, J. (2005) Bioactive microbial metabolites. J Antibiot (Tokyo) 58: 1-26.

Bernard, R., El Ghachi, M., Mengin-Lecreulx, D., Chippaux, M., and Denizot, F. (2005) BcrC from Bacillus subtilis acts as an undecaprenyl pyrophosphate phosphatase in bacitracin resistance. J. Biol. Chem. 280: 28852-28857.

Bernat, B.A., Laughlin, L.T., and Armstrong, R.N. (1997) Fosfomycin resistance protein (FosA) is a manganese metalloglutathione transferase related to glyoxalase I and the extradiol dioxygenases. Biochemistry 36: 3050-3055.

Bhavsar, A.P., Erdman, L.K., Schertzer, J.W., and Brown, E.D. (2004) Teichoic Acid Is an Essential Polymer in Bacillus subtilis That Is Functionally Distinct from Teichuronic Acid. J. Bacteriol. 186: 7865-7873.

Bilwes, A.M., Park, S.-Y., Quezada, C.M., Simon, M.I., and Crane, B.R. (2003) Structure and function of CheA, the histidine kinase central to bacterial chemotaxis. In Histidine Kinases in Signal Transduction. Inouye, M. and Dutta, R. (eds). San Diego, CA: Academic Press, pp. 48-73.

Bobay, B.G., Benson, L., Naylor, S., Feeney, B., Clark, A.C., Goshe, M.B., Strauch, M.A., Thompson, R., and Cavanagh, J. (2004) Evaluation of the DNA binding tendencies of the transition state regulator AbrB. Biochemistry 43: 16106-16118.

Bobay, B.G., Mueller, G.A., Thompson, R.J., Murzin, A.G., Venters, R.A., Strauch, M.A., and Cavanagh, J. (2006) NMR structure of AbhN and comparison with AbrBN: first insights into the DNA binding promiscuity and specificity of AbrB-like transition state regulator proteins. J. Biol. Chem. 281: 21399-21409.

Bordes, P., Wigneshweraraj, S.R., Schumacher, J., Zhang, X., Chaney, M., and Buck, M. (2003) The ATP hydrolyzing transcription activator phage shock protein $\mathrm{F}$ of Escherichia coli: identifying a surface that binds $\sigma^{54}$. Proc Natl Acad Sci U S A 100: 2278-2283.

Branda, S.S., Vik, S., Friedman, L., and Kolter, R. (2005) Biofilms: the matrix revisited. Trends Microbiol 13: 20-26.

Breukink, E., and de Kruijff, B. (2006) Lipid II as a target for antibiotics. Nat Rev Drug Discov 5: 321-332.

Brissette, J.L., Russel, M., Weiner, L., and Model, P. (1990) Phage shock protein, a stress protein of Escherichia coli. Proc Natl Acad Sci U S A 87: 862-866.

Brogden, K.A. (2005) Antimicrobial peptides: pore formers or metabolic inhibitors in bacteria? Nat Rev Microbiol 3: 238-250.

Buelow, D.R., and Raivio, T.L. (2005) Cpx signal transduction Is influenced by a conserved $\mathrm{N}$-terminal domain in the novel inhibitor CpxP and the periplasmic protease DegP. $J$. Bacteriol. 187: 6622-6630.

Bugg, T.D., and Walsh, C.T. (1992) Intracellular steps of bacterial cell wall peptidoglycan biosynthesis: enzymology, antibiotics, and antibiotic resistance. Nat Prod Rep 9: 199215.

Buhrke, T., Lenz, O., Porthun, A., and Friedrich, B. (2004) The $\mathrm{H}_{2}$-sensing complex of Ralstonia eutropha: interaction between a regulatory [NiFe] hydrogenase and a histidine protein kinase. Mol Microbiol 51: 1677-1689.

Burbulys, D., Trach, K.A., and Hoch, J.A. (1991) Initiation of sporulation in B. subtilis is controlled by a multicomponent phosphorelay. Cell 64: 545-552.

Burkholder, W.F., Kurtser, I., and Grossman, A.D. (2001) Replication initiation proteins regulate a developmental checkpoint in Bacillus subtilis. Cell 104: 269-279. 
Butcher, B.G., and Helmann, J.D. (2006) Identification of Bacillus subtilis $\sigma^{\mathrm{W}}$-dependent genes that provide intrinsic resistance to antimicrobial compounds produced by Bacilli. Mol Microbiol 60: 765-782.

Butcher, B.G., Mascher, T., and Helmann, J.D. (2007) Environmental sensing and the role of extracytoplasmic function (ECF) $\sigma \phi \alpha \chi \tau \mathrm{O} \sigma \sigma$. In Bacterial Physiology - a Molecular Approach. El-Sharoud, W.M. (ed). Berlin, Heidelberg: Springer-Verlag GmbH, pp. in press.

Butler, S.L., and Falke, J.J. (1998) Cysteine and Disulfide Scanning Reveals Two Amphiphilic Helices in the Linker Region of the Aspartate Chemoreceptor. Vol. 37, pp. 10746-10756.

Cain, B.D., Norton, P.J., Eubanks, W., Nick, H.S., and Allen, C.M. (1993) Amplification of the bacA gene confers bacitracin resistance to Escherichia coli. J Bacteriol 175: 37843789.

Cao, M., Bernat, B.A., Wang, Z., Armstrong, R.N., and Helmann, J.D. (2001) FosB, a cysteine-dependent fosfomycin resistance protein under the control of $\sigma^{\mathrm{W}}$, an extracytoplasmic-function $\sigma$ factor in Bacillus subtilis. J Bacteriol 183: 2380-2383.

Cao, M., and Helmann, J.D. (2002) Regulation of the Bacillus subtilis bcrC bacitracin resistance gene by two extracytoplasmic function $\sigma$ factors. J Bacteriol 184: 61236129.

Cao, M., Kobel, P.A., Morshedi, M.M., Wu, M.F., Paddon, C., and Helmann, J.D. (2002a) Defining the Bacillus subtilis $\sigma^{\mathrm{W}}$ regulon: a comparative analysis of promoter consensus search, run-off transcription/macroarray analysis (ROMA), and transcriptional profiling approaches. J Mol Biol 316: 443-457.

Cao, M., Wang, T., Ye, R., and Helmann, J.D. (2002b) Antibiotics that inhibit cell wall biosynthesis induce expression of the Bacillus subtilis $\sigma^{\mathrm{W}}$ and $\sigma^{\mathrm{M}}$ regulons. Mol Microbiol 45: 1267-1276.

Cao, M., Salzberg, L., Tsai, C.S., Mascher, T., Bonilla, C., Wang, T., Ye, R.W., MarquezMagana, L., and Helmann, J.D. (2003) Regulation of the Bacillus subtilis extracytoplasmic function protein $\sigma^{\mathrm{Y}}$ and its target promoters. J Bacteriol 185: 48834890.

Cao, M., and Helmann, J.D. (2004) The Bacillus subtilis extracytoplasmic-function $\sigma^{\mathrm{X}}$ factor regulates modification of the cell envelope and resistance to cationic antimicrobial peptides. J Bacteriol 186: 1136-1146.

Chalker, A.F., Ingraham, K.A., Lunsford, R.D., Bryant, A.P., Bryant, J., Wallis, N.G., Broskey, J.P., Pearson, S.C., and Holmes, D.J. (2000) The bacA gene, which determines bacitracin susceptibility in Streptococcus pneumoniae and Staphylococcus aureus, is also required for virulence. Microbiology 146: 1547-1553.

Champness, W. (2000) Actinomycete development, antibiotic production, and phylogeny: questions and challenges. In Prokaryotic Development. Brun, Y.V. and Shimkets, L.J. (eds). Washington, DC: ASM press.

Chatterjee, C., Paul, M., Xie, L., and van der Donk, W.A. (2005) Biosynthesis and mode of action of lantibiotics. Chem Rev 105: 633-684.

Chung, J.D., Stephanopoulos, G., Ireton, K., and Grossman, A.D. (1994) Gene expression in single cells of Bacillus subtilis: evidence that a threshold mechanism controls the initiation of sporulation. J Bacteriol 176: 1977-1984.

Clausen, V.A., Bae, W., Throup, J., Burnham, M.K., Rosenberg, M., and Wallis, N.G. (2003) Biochemical characterization of the first essential two-component signal transduction system from Staphylococcus aureus and Streptococcus pneumoniae. J Mol Microbiol Biotechnol 5: 252-260.

Crater, D.L., and Moran, C.P., Jr. (2001) Identification of a DNA binding region in GerE from Bacillus subtilis. J Bacteriol 183: 4183-4189. 
Crooks, G.E., Hon, G., Chandonia, J.M., and Brenner, S.E. (2004) WebLogo: a sequence logo generator. Genome Res 14: 1188-1190.

D'Costa, V.M., McGrann, K.M., Hughes, D.W., and Wright, G.D. (2006) Sampling the antibiotic resistome. Science 311: 374-377.

D'Elia, M.A., Millar, K.E., Beveridge, T.J., and Brown, E.D. (2006a) Wall teichoic acid polymers are dispensable for cell viability in Bacillus subtilis. J Bacteriol 188: 83138316.

D'Elia, M.A., Pereira, M.P., Chung, Y.S., Zhao, W., Chau, A., Kenney, T.J., Sulavik, M.C., Black, T.A., and Brown, E.D. (2006b) Lesions in teichoic acid biosynthesis in Staphylococcus aureus lead to a lethal gain of function in the otherwise dispensable pathway. J Bacteriol 188: 4183-4189.

Dahl, J.L., Wei, B.Y., and Kadner, R.J. (1997) Protein phosphorylation affects binding of the Escherichia coli transcription activator UhpA to the uhpT promoter. J Biol Chem 272: 1910-1919.

Dartois, V., Djavakhishvili, T., and Hoch, J. (1996) Identification of a membrane protein involved in activation of the KinB pathway to sporulation in Bacillus subtilis. $J$. Bacteriol. 178: 1178-1186.

Dartois, V., Djavakhishvili, T., and Hoch, J.A. (1997a) KapB is a lipoprotein required for KinB signal transduction and activation of the phosphorelay to sporulation in Bacillus subtilis. Mol Microbiol 26: 1097-1108.

Dartois, V., Liu, J., and Hoch, J.A. (1997b) Alterations in the flow of one-carbon units affect KinB-dependent sporulation in Bacillus subtilis. Mol Microbiol 25: 39-51.

Darwin, A.J., and Miller, V.L. (2001) The $p s p$ locus of Yersinia enterocolitica is required for virulence and for growth in vitro when the Ysc type III secretion system is produced. Mol Microbiol 39: 429-445.

Darwin, A.J. (2005) The phage-shock-protein response. Mol Microbiol 57: 621-628.

De Wulf, P., McGuire, A.M., Liu, X., and Lin, E.C. (2002) Genome-wide profiling of promoter recognition by the two-component response regulator CpxR-P in Escherichia coli. J Biol Chem 277: 26652-26661.

Delcour, J., Ferain, T., Deghorain, M., Palumbo, E., and Hols, P. (1999) The biosynthesis and functionality of the cell-wall of lactic acid bacteria. Antonie Van Leeuwenhoek 76: 159-184.

DeLisa, M.P., Lee, P., Palmer, T., and Georgiou, G. (2004) Phage shock protein PspA of Escherichia coli relieves saturation of protein export via the Tat pathway. J Bacteriol 186: $366-373$.

Depardieu, F., Courvalin, P., and Msadek, T. (2003) A six amino acid deletion, partially overlapping the VanSB G2 ATP-binding motif, leads to constitutive glycopeptide resistance in VanB-type Enterococcus faecium. Mol Microbiol 50: 1069-1083.

Derre, I., Rapoport, G., and Msadek, T. (2000) The CtsR regulator of stress response is active as a dimer and specifically degraded in vivo at $37^{\circ} \mathrm{C}$. Mol Microbiol 38: 335-347.

Dmitriev, B., Toukach, F., and Ehlers, S. (2005) Towards a comprehensive view of the bacterial cell wall. Trends Microbiol 13: 569-574.

Dover, L.G., Cerdeno-Tarraga, A.M., Pallen, M.J., Parkhill, J., and Besra, G.S. (2004) Comparative cell wall core biosynthesis in the mycolated pathogens, Mycobacterium tuberculosis and Corynebacterium diphtheriae. FEMS Microbiol Rev 28: 225-250.

Dowson, C.G., Coffey, T.J., and Spratt, B.G. (1994) Origin and molecular epidemiology of penicillin-binding-protein-mediated resistance to $\beta$-lactam antibiotics. Trends Microbiol 2: 361-366.

Dubnau, D., and Losick, R. (2006) Bistability in bacteria. Mol Microbiol 61: 564-572.

Dworkin, J., Jovanovic, G., and Model, P. (2000) The PspA protein of Escherichia coli is a negative regulator of sigma(54)-dependent transcription. J Bacteriol 182: 311-319. 
Economou, A. (1999) Following the leader: bacterial protein export through the Sec pathway. Trends Microbiol 7: 315-320.

Egland, K.A., and Greenberg, E.P. (2000) Conversion of the Vibrio fischeri transcriptional activator, LuxR, to a repressor. J Bacteriol 182: 805-811.

Eldakak, A., and Hulett, F.M. (2007) Cys303 in the Histidine Kinase PhoR Is Crucial for the Phosphotransfer Reaction in the PhoPR Two-Component System in Bacillus subtilis. Vol. 189, pp. 410-421.

Ellermeier, C.D., Hobbs, E.C., Gonzalez-Pastor, J.E., and Losick, R. (2006) A three-protein signaling pathway governing immunity to a bacterial cannibalism toxin. Cell 124: 549-559.

Ellermeier, C.D., and Losick, R. (2006) Evidence for a novel protease governing regulated intramembrane proteolysis and resistance to antimicrobial peptides in Bacillus subtilis. Genes Dev. 20: 1911-1922.

Emmerich, R., Strehler, P., Hennecke, H., and Fischer, H.-M. (2000) An imperfect inverted repeat is critical for DNA binding of the response regulator RegR of Bradyrhizobium japonicum. Nucl. Acids Res. 28: 4166-4171.

Eraso, J.M., and Kaplan, S. (2000) From redox flow to gene regulation: role of the PrrC protein of Rhodobacter sphaeroides 2.4.1. Biochemistry 39: 2052-2062.

Errington, J. (2003) Regulation of endospore formation in Bacillus subtilis. Nat Rev Microbiol 1: 117-126.

Fabret, C., and Hoch, J.A. (1998) A two-component signal transduction system essential for growth of Bacillus subtilis: implications for anti-infective therapy. J. Bacteriol. 180: 6375-6383.

Fawcett, P., Eichenberger, P., Losick, R., and Youngman, P. (2000) The transcriptional profile of early to middle sporulation in Bacillus subtilis. Proc Natl Acad Sci U S A 97: 8063-8068.

Fedtke, I., Kamps, A., Krismer, B., and Götz, F. (2002) The nitrate reductase and nitrite reductase operons and the narT gene of Staphylococcus carnosus are positively controlled by the novel two-component system NreBC. J Bacteriol 184: 6624-6634.

Feng, Z., and Barletta, R.G. (2003) Roles of Mycobacterium smegmatis D-alanine:D-alanine ligase and D-alanine racemase in the mechanisms of action of and resistance to the peptidoglycan inhibitor D-cycloserine. Antimicrob Agents Chemother 47: 283-291.

Ferrell, J.E. (2002) Self-perpetuating states in signal transduction: positive feedback, doublenegative feedback and bistability. Current Opinion in Cell Biology 14: 140-148.

Fleischer, R., Heermann, R., Jung, K., and Hunke, S. (2007) Purification, reconstitution, and characterization of the CpxRAP envelope stress system of Escherichia coli. J Biol Chem 282: 8583-8593.

Foster, S.J., and Popham, D.L. (2002) Structure and synthesis of cell wall, spore cortex, teichoic acid, S-layers and capsules. In Bacillus subtilis and Its Closest Relatives. From Genes to Cells. Sonenshein, A.L., Hoch, J.A. and Losick, R. (eds). Washigton D.C.: ASM Press, pp. 21-41.

Friedrich, B., Buhrke, T., Burgdorf, T., and Lenz, O. (2005) A hydrogen-sensing multiprotein complex controls aerobic hydrogen metabolism in Ralstonia eutropha. Biochem Soc Trans 33: 97-101.

Fujita, M., Gonzalez-Pastor, J.E., and Losick, R. (2005) High- and low-threshold genes in the Spo0A regulon of Bacillus subtilis. J. Bacteriol. 187: 1357-1368.

Gaal, T., Ross, W., Blatter, E.E., Tang, H., Jia, X., Krishnan, V.V., Assa-Munt, N., Ebright, R.H., and Gourse, R.L. (1996) DNA-binding determinants of the alpha subunit of RNA polymerase: novel DNA-binding domain architecture. Genes Dev 10: 16-26.

Galperin, M.Y. (2006) Structural classification of bacterial response regulators: diversity of output domains and domain combinations. J Bacteriol 188: 4169-4182. 
Gao, T., Zhang, X., Ivleva, N.B., Golden, S.S., and Liwang, A. (2007) NMR structure of the pseudo-receiver domain of CikA. Protein Sci 16: 465-475.

Gardete, S., Wu, S.W., Gill, S., and Tomasz, A. (2006) Role of VraSR in antibiotic resistance and antibiotic-induced stress response in Staphylococcus aureus. Antimicrob. Agents Chemother. 50: 3424-3434.

Gardner, T.S., Cantor, C.R., and Collins, J.J. (2000) Construction of a genetic toggle switch in Escherichia coli. Nature 403: 339-342.

Garnerone, A.-M., Cabanes, D., Foussard, M., Boistard, P., and Batut, J. (1999) Inhibition of the FixL sensor kinase by the FixT protein in Sinorhizobium meliloti. J. Biol. Chem. 274: 32500-32506.

Ghuysen, J.M. (1991) Serine beta-lactamases and penicillin-binding proteins. Annu Rev Microbiol 45: 37-67.

Giuliani, A., Pirri, G., and Nicoletto, S.F. (2007) Antimicrobial peptides: an overview of a promising class of therapeutics. Central European Journal of Biology 2: 1-33.

Gon, S., Jourlin-Castelli, C., Theraulaz, L., and Mejean, V. (2001) An unsuspected autoregulatory pathway involving apocytochrome TorC and sensor TorS in Escherichia coli. PNAS 98: 11615-11620.

Gonzalez-Pastor, J.E., Hobbs, E.C., and Losick, R. (2003) Cannibalism by sporulating bacteria. Science 301: 510-513.

Gravesen, A., Sorensen, K., Aarestrup, F.M., and Knochel, S. (2001) Spontaneous nisinresistant Listeria monocytogenes mutants with increased expression of a putative penicillin-binding protein and their sensitivity to various antibiotics. Microb Drug Resist 7: 127-135.

Gravesen, A., Kallipolitis, B., Holmstrom, K., Hoiby, P.E., Ramnath, M., and Knochel, S. (2004) pbp2229-mediated nisin resistance mechanism in Listeria monocytogenes confers cross-protection to class IIa bacteriocins and affects virulence gene expression. Appl. Environ. Microbiol. 70: 1669-1679.

Grebe, T.W., and Stock, J. (1998) Bacterial chemotaxis: the five sensors of a bacterium. Curr Biol 8: R154-157.

Gründling, A., and Schneewind, O. (2007) Synthesis of glycerol phosphate lipoteichoic acid in Staphylococcus aureus. Proc Natl Acad Sci U S A 104: 8478-8483.

Guenzi, E., Gasc, A.M., Sicard, M.A., and Hakenbeck, R. (1994) A two-component signaltransducing system is involved in competence and penicillin susceptibility in laboratory mutants of Streptococcus pneumoniae. Mol Microbiol 12: 505-515.

Guerout-Fleury, A.M., Shazand, K., Frandsen, N., and Stragier, P. (1995) Antibioticresistance cassettes for Bacillus subtilis. Gene 167: 335-336.

Guerout-Fleury, A.M., Frandsen, N., and Stragier, P. (1996) Plasmids for ectopic integration in Bacillus subtilis. Gene 180: 57-61.

Haas, W., Kaushal, D., Sublett, J., Obert, C., and Tuomanen, E.I. (2005) Vancomycin stress response in a sensitive and a tolerant strain of Streptococcus pneumoniae. J. Bacteriol. 187: 8205-8210.

Hakenbeck, R. (1999) $\beta$-lactam-resistant Streptococcus pneumoniae: epidemiology and evolutionary mechanism. Chemotherapy 45: 83-94.

Hall, T.A. (1999) BioEdit: a user-friendly biological sequence alignment editor and analysis program for Windows 95/98/NT. Nucl. Acids. Symp. Ser. 41: 95-98.

Hamoen, L.W., Van Werkhoven, A.F., Bijlsma, J.J.E., Dubnau, D., and Venema, G. (1998) The competence transcription factor of Bacillus subtilis recognizes short A/T-rich sequences arranged in a unique, flexible pattern along the DNA helix. Vol. 12, pp. 1539-1550. 
Hamon, M.A., Stanley, N.R., Britton, R.A., Grossman, A.D., and Lazazzera, B.A. (2004) Identification of AbrB-regulated genes involved in biofilm formation by Bacillus subtilis. Molecular Microbiology 52: 847-860.

Hancock, L.E., and Perego, M. (2004) Systematic Inactivation and Phenotypic Characterization of Two-Component Signal Transduction Systems of Enterococcus faecalis V583. J. Bacteriol. 186: 7951-7958.

Hancock, R.E., and Sahl, H.G. (2006) Antimicrobial and host-defense peptides as new antiinfective therapeutic strategies. Nat Biotechnol 24: 1551-1557.

Hankamer, B.D., Elderkin, S.L., Buck, M., and Nield, J. (2004) Organization of the AAA(+) adaptor protein PspA is an oligomeric ring. J Biol Chem 279: 8862-8866.

Hansen, M.E., Mijakovic, I., Jensen, P.R., and Sibbesen, O. (2007) Use of Bacillus subtilis for the biosynthesis of nisin. In 4th Conference on Functional Genomics of Gram-positive Microorganisms Tirrenia, Pisa, Italy.

Hardie, K.R., Lory, S., and Pugsley, A.P. (1996) Insertion of an outer membrane protein in Escherichia coli requires a chaperone-like protein. Embo J 15: 978-988.

Harwood, C.R., and Cutting, S.M. (1990) Molecular Biological Methods for Bacillus. Chichester: John Wiley \& Sons.

Healy, V.L., Lessard, I.A., Roper, D.I., Knox, J.R., and Walsh, C.T. (2000) Vancomycin resistance in enterococci: reprogramming of the D-ala-D-Ala ligases in bacterial peptidoglycan biosynthesis. Chem Biol 7: R109-119.

Heinrich, J., and Wiegert, T. (2006) YpdC determines site-1 degradation in regulated intramembrane proteolysis of the RsiW anti-sigma factor of Bacillus subtilis. Mol Microbiol 62: 566-579.

Helmann, J.D. (2002) The extracytoplasmic function (ECF) sigma factors. Adv Microb Physiol 46: 47-110.

Helmann, J.D., and Moran, C.P. (2002) RNA polymerase and sigma factors. In Bacillus subtilis and Its Closest Relatives - From Genes to Cells. Sonenshein, A.L., Hoch, J.A. and Losick, R. (eds). Washington D.C.: ASM Press, pp. 289-312.

Helmann, J.D. (2006) Deciphering a complex genetic regulatory network: the Bacillus subtilis $\sigma^{\mathrm{W}}$ protein and intrinsic resistance to antimicrobial compounds. Sci Prog 89: 243-266.

Hernandez, C., Olano, C., Mendez, C., and Salas, J.A. (1993) Characterization of a Streptomyces antibioticus gene cluster encoding a glycosyltransferase involved in oleandomycin inactivation. Gene 134: 139-140.

Hirakawa, H., Nishino, K., Yamada, J., Hirata, T., and Yamaguchi, A. (2003) $\beta$-Lactam resistance modulated by the overexpression of response regulators of two-component signal transduction systems in Escherichia coli. J. Antimicrob. Chemother. 52: 576582.

Höltje, J.V. (1998) Growth of the stress-bearing and shape-maintaining murein sacculus of Escherichia coli. Microbiol Mol Biol Rev 62: 181-203.

Hong, H.J., Paget, M.S., and Buttner, M.J. (2002) A signal transduction system in Streptomyces coelicolor that activates the expression of a putative cell wall glycan operon in response to vancomycin and other cell wall-specific antibiotics. Mol Microbiol 44: 1199-1211.

Horii, T., Kimura, T., Sato, K., Shibayama, K., and Ohta, M. (1999) Emergence of fosfomycin-resistant isolates of Shiga-like toxin-producing Escherichia coli O26. Antimicrob Agents Chemother 43: 789-793.

Horsburgh, M.J., and Moir, A. (1999) $\sigma^{\mathrm{M}}$, an ECF RNA polymerase sigma factor of Bacillus subtilis 168, is essential for growth and survival in high concentrations of salt. $\mathrm{Mol}$ Microbiol 32: 41-50.

Hoskisson, P.A., and Hutchings, M.I. (2006) MtrAB-LpqB: a conserved three-component system in actinobacteria? Trends Microbiol 14: 444-449. 
Hu, Y., Helm, J.S., Chen, L., Ye, X.Y., and Walker, S. (2003) Ramoplanin inhibits bacterial transglycosylases by binding as a dimer to lipid II. J Am Chem Soc 125: 8736-8737.

Huang, X., Decatur, A., Sorokin, A., and Helmann, J.D. (1997) The Bacillus subtilis $\sigma^{\mathrm{X}}$ protein is an extracytoplasmic function sigma factor contributing to survival at high temperature. J Bacteriol 179: 2915-2921.

Huang, X., Fredrick, K.L., and Helmann, J.D. (1998) Promoter recognition by Bacillus subtilis $\sigma^{\mathrm{W}}$ : autoregulation and partial overlap with the $\sigma^{\mathrm{X}}$ regulon. J Bacteriol 180: 3765-3770.

Huang, X., Gaballa, A., Cao, M., and Helmann, J.D. (1999) Identification of target promoters for the Bacillus subtilis extracytoplasmic function $\sigma$ factor, $\sigma^{\mathrm{W}}$. Mol Microbiol 31: 361-371.

Hulko, M., Berndt, F., Gruber, M., Linder, J.U., Truffault, V., Schultz, A., Martin, J., Schultz, J.E., Lupas, A.N., and Coles, M. (2006) The HAMP domain structure implies helix rotation in transmembrane signaling. Cell 126: 929-940.

Hurley, J.H. (2003) GAF domains: cyclic nucleotides come full circle. Sci STKE 2003: PE1.

Hutchings, M.I., Hoskisson, P.A., Chandra, G., and Buttner, M.J. (2004) Sensing and responding to diverse extracellular signals? Analysis of the sensor kinases and response regulators of Streptomyces coelicolor A3(2). Microbiology 150: 2795-2806.

Hutchings, M.I., Hong, H.-J., Leibovitz, E., Sutcliffe, I.C., and Buttner, M.J. (2006a) The $\sigma^{\mathrm{E}}$ cell envelope stress response of Streptomyces coelicolor is influenced by a novel lipoprotein, CseA. J. Bacteriol. 188: 7222-7229.

Hutchings, M.I., Hong, H.J., and Buttner, M.J. (2006b) The vancomycin resistance VanRS two-component signal transduction system of Streptomyces coelicolor. Mol Microbiol 59: 923-935.

Hutter, B., Schaab, C., Albrecht, S., Borgmann, M., Brunner, N.A., Freiberg, C., Ziegelbauer, K., Rock, C.O., Ivanov, I., and Loferer, H. (2004) Prediction of Mechanisms of Action of Antibacterial Compounds by Gene Expression Profiling. Antimicrob. Agents Chemother. 48: 2838-2844.

Hyyryläinen, H.-L., Pietiäinen, M., Lunden, T., Ekman, A., Gardemeister, M., MurtomakiRepo, S., Antelmann, H., Hecker, M., Valmu, L., Sarvas, M., and Kontinen, V.P. (2007) The density of negative charge in the cell wall influences two-component signal transduction in Bacillus subtilis. Microbiology 153: 2126-2136.

Hyyryläinen, H.L., Sarvas, M., and Kontinen, V.P. (2005) Transcriptome analysis of the secretion stress response of Bacillus subtilis. Appl Microbiol Biotechnol 67: 389-396.

Island, M.D., and Kadner, R.J. (1993) Interplay between the membrane-associated UhpB and UhpC regulatory proteins. J Bacteriol 175: 5028-5034.

Ivleva, N.B., Gao, T., LiWang, A.C., and Golden, S.S. (2006) Quinone sensing by the circadian input kinase of the cyanobacterial circadian clock. PNAS 103: 17468-17473.

Jervis, A.J., Thackray, P.D., Houston, C.W., Horsburgh, M.J., and Moir, A. (2007) SigMresponsive genes of Bacillus subtilis and their promoters. J. Bacteriol. 189: 45344538.

Jordan, S., Junker, A., Helmann, J.D., and Mascher, T. (2006) Regulation of LiaRS-dependent gene expression in Bacillus subtilis: Identification of inhibitor proteins, regulator binding sites and target genes of a conserved cell envelope stress-sensing twocomponent system. J Bacteriol 188: 5153-5166.

Jordan, S., Rietkötter, E., Strauch, M.A., Kalamorz, F., Butcher, B.G., Helmann, J.D., and Mascher, T. (2007) LiaRS-dependent gene expression is embedded in transition state regulation in Bacillus subtilis. Microbiology 153: 2530-2540.

Joseph, P., Fichant, G., Quentin, Y., and Denizot, F. (2002) Regulatory relationship of twocomponent and $\mathrm{ABC}$ transport systems and clustering of their genes in the 
Bacillus/Clostridium group, suggest a functional link between them. J Mol Microbiol Biotechnol 4: 503-513.

Joseph, P., Guiseppi, A., Sorokin, A., and Denizot, F. (2004) Characterization of the Bacillus subtilis YxdJ response regulator as the inducer of expression for the cognate ABC transporter YxdLM. Microbiology 150: 2609-2617.

Kadner, R.J. (1995) Expression of the Uhp sugar-phosphate transport system of Escherichia coli. In Two-Component Signal Transduction. Hoch, J.A. and Silhavy, T.J. (eds). Washington, D.C.: ASM press.

Kahan, F.M., Kahan, J.S., Cassidy, P.J., and Kropp, H. (1974) The mechanism of action of fosfomycin (phosphonomycin). Ann N Y Acad Sci 235: 364-386.

Kahne, D., Leimkuhler, C., Lu, W., and Walsh, C. (2005) Glycopeptide and lipoglycopeptide antibiotics. Chem Rev 105: 425-448.

Kallipolitis, B.H., Ingmer, H., Gahan, C.G., Hill, C., and Sogaard-Andersen, L. (2003) CesRK, a Two-Component Signal Transduction System in Listeria monocytogenes, Responds to the Presence of Cell Wall-Acting Antibiotics and Affects \{beta\}-Lactam Resistance. Antimicrob. Agents Chemother. 47: 3421-3429.

Kearns, D.B., and Losick, R. (2005) Cell population heterogeneity during growth of Bacillus subtilis. Vol. 19, pp. 3083-3094.

Kendall, S.L., Movahedzadeh, F., Rison, S.C., Wernisch, L., Parish, T., Duncan, K., Betts, J.C., and Stoker, N.G. (2004) The Mycobacterium tuberculosis dosRS two-component system is induced by multiple stresses. Tuberculosis (Edinb) 84: 247-255.

Kleerebezem, M., and Tommassen, J. (1993) Expression of the $p s p A$ gene stimulates efficient protein export in Escherichia coli. Mol Microbiol 7: 947-956.

Kleerebezem, M., Crielaard, W., and Tommassen, J. (1996) Involvement of stress protein PspA (phage shock protein A) of Escherichia coli in maintenance of the protonmotive force under stress conditions. Embo J 15: 162-171.

Kleihues, L., Lenz, O., Bernhard, M., Buhrke, T., and Friedrich, B. (2000) The $\mathrm{H}_{2}$ sensor of Ralstonia eutropha is a member of the subclass of regulatory [NiFe] hydrogenases. $J$ Bacteriol 182: 2716-2724.

Klein, A.H., Shulla, A., Reimann, S.A., Keating, D.H., and Wolfe, A.J. (2007) The intracellular concentration of acetyl phosphate in Escherichia coli is sufficient for direct phosphorylation of two-component response regulators. J Bacteriol 189: 55745581.

Kobayashi, H., Yamamoto, M., and Aono, R. (1998) Appearance of a stress-response protein, phage-shock protein A, in Escherichia coli exposed to hydrophobic organic solvents. Microbiology 144 ( Pt 2): 353-359.

Kobayashi, K., Ogura, M., Yamaguchi, H., Yoshida, K., Ogasawara, N., Tanaka, T., and Fujita, Y. (2001) Comprehensive DNA microarray analysis of Bacillus subtilis twocomponent regulatory systems. J Bacteriol 183: 7365-7370.

Kobayashi, K. (2007) Bacillus subtilis pellicle formation proceeds through genetically defined morphological changes. J Bacteriol 189: 4920-4931.

Koch, A.L. (2003) Bacterial wall as target for attack: past, present, and future research. Clin Microbiol Rev 16: 673-687.

Kovács, M., Halfmann, A., Fedtke, I., Heintz, M., Peschel, A., Vollmer, W., Hakenbeck, R., and Brückner, R. (2006) A functional dlt operon, encoding proteins required for incorporation of D-alanine in teichoic acids in Gram-positive bacteria, confers resistance to cationic antimicrobial peptides in Streptococcus pneumoniae. J. Bacteriol. 188: 5797-5805.

Kuroda, M., Kuwahara-Arai, K., and Hiramatsu, K. (2000) Identification of the up- and down-regulated genes in vancomycin-resistant Staphylococcus aureus strains Mu3 and 
Mu50 by cDNA differential hybridization method. Biochem Biophys Res Commun 269: 485-490.

Kuroda, M., Kuroda, H., Oshima, T., Takeuchi, F., Mori, H., and Hiramatsu, K. (2003) Twocomponent system VraSR positively modulates the regulation of cell-wall biosynthesis pathway in Staphylococcus aureus. Mol Microbiol 49: 807-821.

Lambert, M.P., and Neuhaus, F.C. (1972) Mechanism of D-cycloserine action: alanine racemase from Escherichia coli W. J Bacteriol 110: 978-987.

Lange, R., Wagner, C., de Saizieu, A., Flint, N., Molnos, J., Stieger, M., Caspers, P., Kamber, M., Keck, W., and Amrein, K.E. (1999) Domain organization and molecular characterization of 13 two-component systems identified by genome sequencing of Streptococcus pneumoniae. Gene 237: 223-234.

Lazar, K., and Walker, S. (2002) Substrate analogues to study cell-wall biosynthesis and its inhibition. Curr Opin Chem Biol 6: 786-793.

Lazarevic, V., Abellan, F.-X., Moller, S.B., Karamata, D., and Mauel, C. (2002) Comparison of ribitol and glycerol teichoic acid genes in Bacillus subtilis W23 and 168: identical function, similar divergent organization, but different regulation. Microbiology 148 : 815-824.

Lenz, O., and Friedrich, B. (1998) A novel multicomponent regulatory system mediates H2 sensing in Alcaligenes eutrophus. Proc Natl Acad Sci U S A 95: 12474-12479.

Li, M., Lai, Y., Villaruz, A.E., Cha, D.J., Sturdevant, D.E., and Otto, M. (2007) Grampositive three-component antimicrobial peptide-sensing system. Proc Natl Acad Sci U $S$ A 104: 9469-9474.

Liger, D., Graille, M., Zhou, C.Z., Leulliot, N., Quevillon-Cheruel, S., Blondeau, K., Janin, J., and van Tilbeurgh, H. (2004) Crystal structure and functional characterization of yeast YLR011wp, an enzyme with NAD(P)H-FMN and ferric iron reductase activities. $J$ Biol Chem 279: 34890-34897.

Lonetto, M.A., Brown, K.L., Rudd, K.E., and Buttner, M.J. (1994) Analysis of the Streptomyces coelicolor sigE gene reveals the existence of a subfamily of eubacterial RNA polymerase sigma factors involved in the regulation of extracytoplasmic functions. Proc Natl Acad Sci U S A 91: 7573-7577.

Majdalani, N., and Gottesman, S. (2005) The Rcs phosphorelay: a complex signal transduction system. Annu Rev Microbiol 59: 379-405.

Mandin, P., Fsihi, H., Dussurget, O., Vergassola, M., Milohanic, E., Toledo-Arana, A., Lasa, I., Johansson, J., and Cossart, P. (2005) VirR, a response regulator critical for Listeria monocytogenes virulence. Mol Microbiol 57: 1367-1380.

Martinez, B., Zomer, A.L., Rodriguez, A., Kok, J., and Kuipers, O.P. (2007) Cell envelope stress induced by the bacteriocin Lcn972 is sensed by the lactococcal two-component system CesSR. Molecular Microbiology 64: 473-486.

Martinez, S.E., Beavo, J.A., and Hol, W.G.J. (2002) GAF domains: two-billion-year-old molecular switches that bind cyclic nucleotides. Mol. Interv. 2: 317-323.

Mascher, T., Margulis, N.G., Wang, T., Ye, R.W., and Helmann, J.D. (2003) Cell wall stress responses in Bacillus subtilis: the regulatory network of the bacitracin stimulon. Mol Microbiol 50: 1591-1604.

Mascher, T., Zimmer, S.L., Smith, T.A., and Helmann, J.D. (2004) Antibiotic-inducible promoter regulated by the cell envelope stress-sensing two-component system LiaRS of Bacillus subtilis. Antimicrob Agents Chemother 48: 2888-2896.

Mascher, T. (2006a) Intramembrane-sensing histidine kinases: a new family of cell envelope stress sensors in Firmicutes bacteria. FEMS Microbiol Lett: in press.

Mascher, T. (2006b) Intramembrane-sensing histidine kinases: a new family of cell envelope stress sensors in Firmicutes bacteria. FEMS Microbiology Letters 264: 133-144. 
Mascher, T., Helmann, J.D., and Unden, G. (2006) Stimulus perception in bacterial signaltransducing histidine kinases. Microbiol Mol Biol Rev 90: 910-938.

Mascher, T., Hachmann, A.-B., and Helmann, J.D. (2007) Regulatory overlap and functional redundancy among Bacillus subtilis extracytoplasmic function (ECF) $\sigma$ factors. $J$. Bacteriol. 189: 6919-6927.

Matias, V.R., and Beveridge, T.J. (2005) Cryo-electron microscopy reveals native polymeric cell wall structure in Bacillus subtilis 168 and the existence of a periplasmic space. Mol Microbiol 56: 240-251.

Matias, V.R., and Beveridge, T.J. (2006) Native cell wall organization shown by cryoelectron microscopy confirms the existence of a periplasmic space in Staphylococcus aureus. J Bacteriol 188: 1011-1021.

Matsuo, H., Kumagai, T., Mori, K., and Sugiyama, M. (2003) Molecular cloning of a Dcycloserine resistance gene from D-cycloserine-producing Streptomyces garyphalus. $J$ Antibiot (Tokyo) 56: 762-767.

McAleese, F., Wu, S.W., Sieradzki, K., Dunman, P., Murphy, E., Projan, S., and Tomasz, A. (2006) Overexpression of genes of the cell wall stimulon in clinical isolates of Staphylococcus aureus exhibiting vancomycin-intermediate- S. aureus-pype resistance to vancomycin. J. Bacteriol. 188: 1120-1133.

McNeil, M.M., and Brown, J.M. (1994) The medically important aerobic actinomycetes: epidemiology and microbiology. Clin Microbiol Rev 7: 357-417.

Meehl, M., Herbert, S., Götz, F., and Cheung, A. (2007) Interaction of the GraRS twocomponent system with the VraFG ABC transporter to support vancomycinintermediate resistance in Staphylococcus aureus. Antimicrob Agents Chemother 51: 2679-2689.

Miller, J.H. (1972) Experiments in molecular genetics. Cold Spring Harbor, New York: Cold Spring Harbor Laboratory.

Ming, L.-J., and Epperson, J.D. (2002) Metal binding and structure-activity relationship of the metalloantibiotic peptide bacitracin. Journal of Inorganic Biochemistry 91: 46-58.

Minnig, K., Barblan, J.L., Kehl, S., Moller, S.B., and Mauel, C. (2003) In Bacillus subtilis W23, the duet $\sigma^{\mathrm{X}} \sigma^{\mathrm{M}}$, two sigma factors of the extracytoplasmic function subfamily, are required for septum and wall synthesis under batch culture conditions. Mol Microbiol 49: 1435-1447.

Minnig, K., Lazarevic, V., Soldo, B., and Mauel, C. (2005) Analysis of teichoic acid biosynthesis regulation reveals that the extracytoplasmic function sigma factor $\sigma^{\mathrm{M}}$ is induced by phosphate depletion in Bacillus subtilis W23. Microbiology 151: 30413049.

Miyake, K., and Iijima, S. (2004) Bacterial capsular polysaccharide and sugar transferases. Adv Biochem Eng Biotechnol 90: 89-111.

Model, P., Jovanovic, G., and Dworkin, J. (1997) The Escherichia coli phage-shock-protein (psp) operon. Mol Microbiol 24: 255-261.

Msadek, T. (1999) When the going gets tough: survival strategies and environmental signaling networks in Bacillus subtilis. Trends Microbiol 7: 201-207.

Münch, R., Hiller, K., Barg, H., Heldt, D., Linz, S., Wingender, E., and Jahn, D. (2003) PRODORIC: prokaryotic database of gene regulation. Nucl. Acids Res. 31: 266-269.

Münch, R., Hiller, K., Grote, A., Scheer, M., Klein, J., Schobert, M., and Jahn, D. (2005) Virtual Footprint and PRODORIC: an integrative framework for regulon prediction in prokaryotes. Bioinformatics 21: 4187-4189.

Mutsuda, M., Michel, K.P., Zhang, X., Montgomery, B.L., and Golden, S.S. (2003) Biochemical properties of CikA, an unusual phytochrome-like histidine protein kinase that resets the circadian clock in Synechococcus elongatus PCC 7942. J Biol Chem 278: 19102-19110. 
Myhre, A.E., Aasen, A.O., Thiemermann, C., and Wang, J.E. (2006) Peptidoglycan - an endotoxin in its own right? Shock 25: 227-235.

Nagakubo, S., Nishino, K., Hirata, T., and Yamaguchi, A. (2002) The putative response regulator BaeR stimulates multidrug resistance of Escherichia coli via a novel multidrug exporter system, MdtABC. J Bacteriol 184: 4161-4167.

Nakano, S., Nakano, M.M., Zhang, Y., Leelakriangsak, M., and Zuber, P. (2003) A regulatory protein that interferes with activator-stimulated transcription in bacteria. Proc Natl Acad Sci U S A 100: 4233-4238.

Neuhaus, F.C., and Lynch, J.L. (1964) The enzymatic synthesis of D-alanyl-D-alanine. 3. On the inhibition of D-alanyl-D-alanine synthetase by the antibiotic D-cycloserine. Biochemistry 3: 471-480.

Neumüller, A.M., Konz, D., and Marahiel, M.A. (2001) The two-component regulatory system BacRS is associated with bacitracin 'self-resistance' of Bacillus licheniformis ATCC 10716. Eur J Biochem 268: 3180-3189.

Nwosu, V.C. (2001) Antibiotic resistance with particular reference to soil microorganisms. Res Microbiol 152: 421-430.

O'Connell-Motherway, M., van Sinderen, D., Morel-Deville, F., Fitzgerald, G.F., Ehrlich, S.D., and Morel, P. (2000) Six putative two-component regulatory systems isolated from Lactococcus lactis subsp. cremoris MG1363. Microbiology 146: 935-947.

O'Riordan, K., and Lee, J.C. (2004) Staphylococcus aureus capsular polysaccharides. Clin Microbiol Rev 17: 218-234.

Ogura, M., Shimane, K., Asai, K., Ogasawara, N., and Tanaka, T. (2003) Binding of response regulator DegU to the aprE promoter is inhibited by RapG, which is counteracted by extracellular PhrG in Bacillus subtilis. Mol Microbiol 49: 1685-1697.

Ogura, M., Matsuzawa, A., Yoshikawa, H., and Tanaka, T. (2004) Bacillus subtilis SalA (YbaL) negatively regulates expression of scoC, which encodes the repressor for the alkaline exoprotease gene, aprE. J Bacteriol 186: 3056-3064.

Ohki, R., Giyanto, Tateno, K., Masuyama, W., Moriya, S., Kobayashi, K., and Ogasawara, N. (2003a) The BceRS two-component regulatory system induces expression of the bacitracin transporter, BceAB, in Bacillus subtilis. Mol Microbiol 49: 1135-1144.

Ohki, R., Tateno, K., Okada, Y., Okajima, H., Asai, K., Sadaie, Y., Murata, M., and Aiso, T. (2003b) A bacitracin-resistant Bacillus subtilis gene encodes a homologue of the membrane-spanning subunit of the Bacillus licheniformis ABC transporter. $J$. Bacteriol. 185: 51-59.

Ozbudak, E.M., Thattai, M., Lim, H.N., Shraiman, B.I., and van Oudenaarden, A. (2004) Multistability in the lactose utilization network of Escherichia coli. Nature 427: 737740.

Paget, M.S., Chamberlin, L., Atrih, A., Foster, S.J., and Buttner, M.J. (1999a) Evidence that the extracytoplasmic function sigma factor $\sigma^{\mathrm{E}}$ is required for normal cell wall structure in Streptomyces coelicolor A3(2). J Bacteriol 181: 204-211.

Paget, M.S., Leibovitz, E., and Buttner, M.J. (1999b) A putative two-component signal transduction system regulates $\sigma^{\mathrm{E}}$, a sigma factor required for normal cell wall integrity in Streptomyces coelicolor A3(2). Mol Microbiol 33: 97-107.

Palumbi, S.R. (2001) Humans as the world's greatest evolutionary force. Science 293: 17861790.

Pao, G.M., Tam, R., Lipschitz, L.S., and Saier, M.H., Jr. (1994) Response regulators: structure, function and evolution. Res Microbiol 145: 356-362.

Parkinson, J.S. (1993) Signal transduction schemes of bacteria. Cell 73: 857-871.

Paul, E.A., and Clark, F.E. (1996) Soil Microbiology and Biochemistry. San Diego, CA (USA): Academic Press. 
Perego, M., Glaser, P., Minutello, A., Strauch, M.A., Leopold, K., and Fischer, W. (1995) Incorporation of D-alanine into lipoteichoic acid and wall teichoic acid in Bacillus subtilis. Identification of genes and regulation. J Biol Chem 270: 15598-15606.

Peschel, A., Otto, M., Jack, R.W., Kalbacher, H., Jung, G., and Götz, F. (1999) Inactivation of the dlt operon in Staphylococcus aureus confers sensitivity to defensins, protegrins, and other antimicrobial peptides. J. Biol. Chem. 274: 8405-8410.

Peters, J.E., and Craig, N.L. (2001) Tn7: smarter than we thought. Nat Rev Mol Cell Biol 2: 806-814.

Petersohn, A., Brigulla, M., Haas, S., Hoheisel, J.D., Volker, U., and Hecker, M. (2001) Global analysis of the general stress response of Bacillus subtilis. J Bacteriol 183: 5617-5631.

Phillips, Z.E., and Strauch, M.A. (2002) Bacillus subtilis sporulation and stationary phase gene expression. Cell Mol Life Sci 59: 392-402.

Pietiäinen, M., Gardemeister, M., Mecklin, M., Leskela, S., Sarvas, M., and Kontinen, V.P. (2005) Cationic antimicrobial peptides elicit a complex stress response in Bacillus subtilis that involves ECF-type sigma factors and two-component signal transduction systems. Microbiology 151: 1577-1592.

Pietiäinen, M., Francois, P., Schrenzel, J., and Kontinen, V.P. (2007) DNA microarray analysis of the response of Staphylococcus aureus to cationic antimicrobial peptides. In 4th Conference on Functional Genomics of Gram-positive Microorganisms Tirrenia, Pisa, Italy.

Piggot, P.J., and Hilbert, D.W. (2004) Sporulation of Bacillus subtilis. Curr Opin Microbiol 7: 579-586.

Podlesek, Z., Comino, A., Herzog-Velikonja, B., Zgur-Bertok, D., Komel, R., and Grabnar, M. (1995) Bacillus licheniformis bacitracin-resistance ABC transporter: relationship to mammalian multidrug resistance. Mol Microbiol 16: 969-976.

Pollock, T.J., Thorne, L., Yamazaki, M., Mikolajczak, M.J., and Armentrout, R.W. (1994) Mechanism of bacitracin resistance in gram-negative bacteria that synthesize exopolysaccharides. J Bacteriol 176: 6229-6237.

Poole, K. (2004) Resistance to $\beta$-lactam antibiotics. Cell Mol Life Sci 61: 2200-2223.

Poole, K. (2005) Efflux-mediated antimicrobial resistance. J Antimicrob Chemother 56: 2051.

Price, C.W. (2002) General stress response. In Bacillus subtilis and Its Closest Relatives From Genes to Cells. Sonenshein, A.L., Hoch, J.A. and Losick, R. (eds). Washington D.C.: ASM Press.

Ptashne, M. (2005) Regulation of transcription: from lambda to eukaryotes. Trends in Biochemical Sciences 30: 275-279.

Qian, Q., Lee, C.Y., Helmann, J.D., and Strauch, M.A. (2002) AbrB is a regulator of the $\sigma^{\mathrm{W}}$ regulon in Bacillus subtilis. FEMS Microbiol Lett 211: 219-223.

Qiu, J., and Helmann, J.D. (2001) The -10 region is a key promoter specificity determinant for the Bacillus subtilis extracytoplasmic-function $\sigma$ factors $\sigma^{\mathrm{X}}$ and $\sigma^{\mathrm{W}}$. J. Bacteriol. 183: 1921-1927.

Raffa, R.G., and Raivio, T.L. (2002) A third envelope stress signal transduction pathway in Escherichia coli. Molecular Microbiology 45: 1599-1611.

Raivio, T.L. (2005) Envelope stress responses and Gram-negative bacterial pathogenesis. Molecular Microbiology 56: 1119-1128.

Rhodius, V.A., Suh, W.C., Nonaka, G., West, J., and Gross, C.A. (2006) Conserved and variable functions of the $\sigma^{\mathrm{E}}$ stress response in related genomes. PLoS Biol 4: e2.

Rietkötter, E., and Mascher, T. (2007) Tunnel vision - bacitracin perception in Bacillus subtilis. In 4th Conference on Functional Genomics of Gram-Positive Microorganisms Tirrenia, Pisa, Italy. 
Rowley, G., Spector, M., Kormanec, J., and Roberts, M. (2006) Pushing the envelope: extracytoplasmic stress responses in bacterial pathogens. Nat Rev Microbiol 4: 383394.

Ruiz, N., and Silhavy, T.J. (2005) Sensing external stress: watchdogs of the Escherichia coli cell envelope. Curr Opin Microbiol 8: 122-126.

Sambrook, J., and Russell, D.W. (2001) Molecular Cloning - a laboratory manual. Cold Spring Harbor, N.Y.: Cold Spring Harbor Laboratory Press.

Santelli, E., Liddington, R.C., Mohan, M.A., Hoch, J.A., and Szurmant, H. (2007) The crystal structure of Bacillus subtilis YycI reveals a common fold for two members of an unusual class of sensor histidine kinase regulatory proteins. J Bacteriol 189: 32903295.

Sardiwal, S., Kendall, S.L., Movahedzadeh, F., Rison, S.C., Stoker, N.G., and Djordjevic, S. (2005) A GAF domain in the hypoxia/NO-inducible Mycobacterium tuberculosis DosS protein binds haem. J Mol Biol 353: 929-936.

Schaeffer, P., Millet, J., and Aubert, J.P. (1965) Catabolic repression of bacterial sporulation. Proc Natl Acad Sci U S A 54: 704-711.

Schäffer, C., and Messner, P. (2005) The structure of secondary cell wall polymers: how Gram-positive bacteria stick their cell walls together. Microbiology 151: 643-651.

Schöbel, S., Zellmeier, S., Schumann, W., and Wiegert, T. (2004) The Bacillus subtilis $\sigma^{\mathrm{W}}$ anti-sigma factor RsiW is degraded by intramembrane proteolysis through YluC. Mol Microbiol 52: 1091-1105.

Schultz, J., Milpetz, F., Bork, P., and Ponting, C.P. (1998) SMART, a simple modular architecture research tool: identification of signaling domains. Proc Natl Acad Sci U S A 95: 5857-5864.

Schwartz, E., Gerischer, U., and Friedrich, B. (1998) Transcriptional regulation of Alcaligenes eutrophus hydrogenase genes. J Bacteriol 180: 3197-3204.

Shafikhani, S.H., Partovi, A.A., and Leighton, T. (2003) Catabolite-induced repression of sporulation in Bacillus subtilis. Curr Microbiol 47: 300-308.

Sherman, D.R., Voskuil, M., Schnappinger, D., Liao, R., Harrell, M.I., and Schoolnik, G.K. (2001) Regulation of the Mycobacterium tuberculosis hypoxic response gene encoding $\alpha$-crystallin. Proc Natl Acad Sci U S A 98: 7534-7539.

Shimoda, N., Toyoda-Yamamoto, A., Aoki, S., and Machida, Y. (1993) Genetic evidence for an interaction between the VirA sensor protein and the ChvE sugar-binding protein of Agrobacterium. J Biol Chem 268: 26552-26558.

Silver, L.L. (2003) Novel inhibitors of bacterial cell wall synthesis. Curr Opin Microbiol 6: 431-438.

Silver, L.L. (2006) Does the cell wall of bacteria remain a viable source of targets for novel antibiotics? Biochem Pharmacol 71: 996-1005.

Sleytr, U.B., Huber, C., Ilk, N., Pum, D., Schuster, B., and Egelseer, E.M. (2007) S-layers as a tool kit for nanobiotechnological applications. FEMS Microbiol Lett 267: 131-144.

Smits, W.K., Eschevins, C.C., Susanna, K.A., Bron, S., Kuipers, O.P., and Hamoen, L.W. (2005) Stripping Bacillus: ComK auto-stimulation is responsible for the bistable response in competence development. Vol. 56, pp. 604-614.

Smits, W.K., Kuipers, O.P., and Veening, J.W. (2006) Phenotypic variation in bacteria: the role of feedback regulation. Nat Rev Microbiol 4: 259-271.

Sobral, R.G., Jones, A.E., Des Etages, S.G., Dougherty, T.J., Peitzsch, R.M., Gaasterland, T., Ludovice, A.M., de Lencastre, H., and Tomasz, A. (2007) Extensive and genome-wide changes in the transcription profile of Staphylococcus aureus induced by modulating the transcription of the cell wall synthesis gene murF. J. Bacteriol. 189: 2376-2391. 
Stanley, N.R., Britton, R.A., Grossman, A.D., and Lazazzera, B.A. (2003) Identification of catabolite repression as a physiological regulator of biofilm formation by Bacillus subtilis by use of DNA microarrays. J Bacteriol 185: 1951-1957.

Staron, A., Sofia, H.J., Liesegang, H., and Mascher, T. (2007) A comparative genomics perspective on the ECF $\sigma$ factor protein family: Classification and functional predictions. In American Society for Microbiology, $107^{\text {th }}$ General Meeting Toronto, Canada.

Stein, T. (2005) Bacillus subtilis antibiotics: structures, syntheses and specific functions. Mol Microbiol 56: 845-857.

Steinmetz, M., and Richter, R. (1994) Easy cloning of mini-Tn10 insertions from the Bacillus subtilis chromosome. J Bacteriol 176: 1761-1763.

Stone, K.J., and Strominger, J.L. (1971) Mechanism of action of bacitracin: complexation with metal ion and C 55 -isoprenyl pyrophosphate. Proc Natl Acad Sci U S A 68: 3223-3227.

Storm, D.R., and Strominger, J.L. (1973) Complex formation between bacitracin peptides and isoprenyl pyrophosphates. The specificity of lipid-peptide interactions. J Biol Chem 248: 3940-3945.

Storz, G., and Hengge-Aronis, R. (2000) Bacterial stress response. Washington, D.C.: ASM press.

Strauch, M., Webb, V., Spiegelman, G., and Hoch, J.A. (1990) The SpoOA protein of Bacillus subtilis is a repressor of the abrB gene. Proc Natl Acad Sci U S A 87: 18011805.

Strauch, M.A., Spiegelman, G.B., Perego, M., Johnson, W.C., Burbulys, D., and Hoch, J.A. (1989) The transition state transcription regulator $a b r B$ of Bacillus subtilis is a DNA binding protein. Embo $J$ 8: 1615-1621.

Strominger, J.L., and Tipper, D.J. (1965) Bacterial cell wall synthesis and structure in relation to the mechanism of action of penicillins and other antibacterial agents. Am J Med 39: 708-721.

Stülke, J., Martin-Verstraete, I., Zagorec, M., Rose, M., Klier, A., and Rapoport, G. (1997) Induction of the Bacillus subtilis ptsGHI operon by glucose is controlled by a novel antiterminator, GlcT. Mol Microbiol 25: 65-78.

Suarez, J.E., and Mendoza, M.C. (1991) Plasmid-encoded fosfomycin resistance. Antimicrob Agents Chemother 35: 791-795.

Sun, J., Zheng, L., Landwehr, C., Yang, J., and Ji, Y. (2005) Identification of a novel essential two-component signal transduction system, YhcSR, in Staphylococcus aureus. J. Bacteriol. 187: 7876-7880.

Suzuki, Y., Yoda, T., Ruhul, A., and Sugiura, W. (2001) Molecular cloning and characterization of the gene coding for azoreductase from Bacillus sp. OY1-2 isolated from soil. J. Biol. Chem. 276: 9059-9065.

Swem, D.L., Swem, L.R., Setterdahl, A., and Bauer, C.E. (2005) Involvement of SenC in assembly of cytochrome c oxidase in Rhodobacter capsulatus. J. Bacteriol. 187: 80818087.

Szurmant, H., Nelson, K., Kim, E.-J., Perego, M., and Hoch, J.A. (2005) YycH regulates the activity of the essential YycFG two-component system in Bacillus subtilis. $J$. Bacteriol. 187: 5419-5426.

Szurmant, H., Zhao, H., Mohan, M.A., Hoch, J.A., and Varughese, K.I. (2006) The crystal structure of $\mathrm{YycH}$ involved in the regulation of the essential YycFG two-component system in Bacillus subtilis reveals a novel tertiary structure. Protein Sci 15: 929-934.

Szurmant, H., Mohan, M.A., Imus, P.M., and Hoch, J.A. (2007a) YycH and YycI interact to regulate the essential YycFG two-component system in Bacillus subtilis. J Bacteriol 189: $3280-3289$. 
Szurmant, H., T., F., and Hoch, J.A. (2007b) Regulation of the essential YycFG twocomponent system in Bacillus subtilis. In 4th Conference on Functional Genomics of Gram-Positive Microorganisms Tirrenia, Pisa, Italy.

Talaat, A.M., Howard, S.T., Hale IV, W., Lyons, R., Garner, H., and Johnston, S.A. (2002) Genomic DNA standards for gene expression profiling in Mycobacterium tuberculosis. Nucl. Acids Res. 30: e104-e109.

Tam le, T., Eymann, C., Albrecht, D., Sietmann, R., Schauer, F., Hecker, M., and Antelmann, H. (2006) Differential gene expression in response to phenol and catechol reveals different metabolic activities for the degradation of aromatic compounds in Bacillus subtilis. Environ Microbiol 8: 1408-1427.

Thackray, P.D., and Moir, A. (2003) SigM, an extracytoplasmic function sigma factor of Bacillus subtilis, is activated in response to cell wall antibiotics, ethanol, heat, acid, and superoxide stress. J Bacteriol 185: 3491-3498.

Thedieck, K., Hain, T., Mohamed, W., Tindall, B.J., Nimtz, M., Chakraborty, T., Wehland, J., and Jansch, L. (2006) The MprF protein is required for lysinylation of phospholipids in listerial membranes and confers resistance to cationic antimicrobial peptides (CAMPs) on Listeria monocytogenes. Mol Microbiol 62: 1325-1339.

Thompson, C.J., Fink, D., and Nguyen, L.D. (2002) Principles of microbial alchemy: insights from the Streptomyces coelicolor genome sequence. Genome Biol 3: REVIEWS1020.

Trach, K.A., and Hoch, J.A. (1993) Multisensory activation of the phosphorelay initiating sporulation in Bacillus subtilis: identification and sequence of the protein kinase of the alternate pathway. Mol Microbiol 8: 69-79.

Tsuda, H., Yamashita, Y., Shibata, Y., Nakano, Y., and Koga, T. (2002) Genes involved in bacitracin resistance in Streptococcus mutans. Antimicrob. Agents Chemother. 46: 3756-3764.

Turgay, K., and Marahiel, M.A. (1995) The gtcRS operon coding for two-component system regulatory proteins is located adjacent to the grs operon of Bacillus brevis. DNA Seq 5: 283-290.

Vagner, V., Dervyn, E., and Ehrlich, S.D. (1998) A vector for systematic gene inactivation in Bacillus subtilis. Microbiology 144 ( Pt 11): 3097-3104.

van Sinderen, D., Luttinger, A., Kong, L., Dubnau, D., Venema, G., and Hamoen, L. (1995) comK encodes the competence transcription factor, the key regulatory protein for competence development in Bacillus subtilis. Mol Microbiol 15: 455-462.

Veening, J.-W., Hamoen, L.W., and Kuipers, O.P. (2005) Phosphatases modulate the bistable sporulation gene expression pattern in Bacillus subtilis. Vol. 56, pp. 1481-1494.

Veiga, P., Bulbarela-Sampieri, C., Furlan, S., Maisons, A., Chapot-Chartier, M.P., Erkelenz, M., Mervelet, P., Noirot, P., Frees, D., Kuipers, O.P., Kok, J., Gruss, A., Buist, G., and Kulakauskas, S. (2007) SpxB regulates $O$-acetylation-dependent resistance of Lactococcus lactis peptidoglycan to hydrolysis. J Biol Chem 282: 19342-19354.

Vellanoweth, R.L., and Rabinowitz, J.C. (1992) The influence of ribosome-binding-site elements on translational efficiency in Bacillus subtilis and Escherichia coli in vivo. Mol Microbiol 6: 1105-1114.

Vicente, M., Hodgson, J., Massidda, O., Tonjum, T., Henriques-Normark, B., and Ron, E.Z. (2006) The fallacies of hope: will we discover new antibiotics to combat pathogenic bacteria in time? FEMS Microbiol Rev 30: 841-852.

Vollmer, W., and Höltje, J.V. (2004) The architecture of the murein (peptidoglycan) in gramnegative bacteria: vertical scaffold or horizontal layer(s)? J Bacteriol 186: 5978-5987.

Voskuil, M.I., Schnappinger, D., Visconti, K.C., Harrell, M.I., Dolganov, G.M., Sherman, D.R., and Schoolnik, G.K. (2003) Inhibition of respiration by nitric oxide induces a Mycobacterium tuberculosis dormancy program. J Exp Med 198: 705-713. 
Vrancken, K., De Keersmaeker, S., Geukens, N., Lammertyn, E., Anne, J., and Van Mellaert, L. (2007) pspA overexpression in Streptomyces lividans improves both Sec- and Tatdependent protein secretion. Appl Microbiol Biotechnol 73: 1150-1157.

Wach, A. (1996) PCR-synthesis of marker cassettes with long flanking homology regions for gene disruptions in S. cerevisiae. Yeast 12: 259-265.

Walker, S., Chen, L., Hu, Y., Rew, Y., Shin, D., and Boger, D.L. (2005) Chemistry and Biology of Ramoplanin: A Lipoglycodepsipeptide with Potent Antibiotic Activity. Chem Rev 105: 449-476.

Walsh, C. (2003) Antibiotics - actions, origins, resistance. Washington, D.C.: ASM press.

Walsh, C.T., Fisher, S.L., Park, I.S., Prahalad, M., and Wu, Z. (1996) Bacterial resistance to vancomycin: five genes and one missing hydrogen bond tell the story. Chem Biol 3: 21-28.

Walsh, T.R., and Howe, R.A. (2002) The prevalence and mechanisms of vancomycin resistance in Staphylococcus aureus. Annu Rev Microbiol 56: 657-675.

Wang, L., Grau, R., Perego, M., and Hoch, J.A. (1997) A novel histidine kinase inhibitor regulating development in Bacillus subtilis. Genes Dev. 11: 2569-2579.

Wecke, T., Veith, B., Ehrenreich, A., and Mascher, T. (2006) Cell envelope stress response in Bacillus licheniformis: Integrating comparative genomics, transcriptional profiling, and regulon mining to decipher a complex regulatory network. J. Bacteriol. 188: 7500-7511.

Weidenmaier, C., Kokai-Kun, J.F., Kristian, S.A., Chanturiya, T., Kalbacher, H., Gross, M., Nicholson, G., Neumeister, B., Mond, J.J., and Peschel, A. (2004) Role of teichoic acids in Staphylococcus aureus nasal colonization, a major risk factor in nosocomial infections. Nat Med 10: 243-245.

Weidenmaier, C., Peschel, A., Xiong, Y.Q., Kristian, S.A., Dietz, K., Yeaman, M.R., and Bayer, A.S. (2005) Lack of wall teichoic acids in Staphylococcus aureus leads to reduced interactions with endothelial cells and to attenuated virulence in a rabbit model of endocarditis. J Infect Dis 191: 1771-1777.

Weiner, L., and Model, P. (1994) Role of an Escherichia coli stress-response operon in stationary-phase survival. Proc Natl Acad Sci U S A 91: 2191-2195.

Weston, L.A., and Kadner, R.J. (1988) Role of $u h p$ genes in expression of the Escherichia coli sugar-phosphate transport system. J Bacteriol 170: 3375-3383.

Wiegert, T., Homuth, G., Versteeg, S., and Schumann, W. (2001) Alkaline shock induces the Bacillus subtilis $\sigma^{\mathrm{W}}$ regulon. Mol Microbiol 41: 59-71.

Wilke, M.S., Lovering, A.L., and Strynadka, N.C. (2005) $\beta$-lactam antibiotic resistance: a current structural perspective. Curr Opin Microbiol 8: 525-533.

Williams, T., Bauer, S., Beier, D., and Kuhn, M. (2005) Construction and characterization of Listeria monocytogenes mutants with in-frame deletions in the response regulator genes identified in the genome sequence. Infect. Immun. 73: 3152-3159.

$\mathrm{Xu}, \mathrm{K}$. , and Strauch, M.A. (1996) In vitro selection of optimal AbrB-binding sites: comparison to known in vivo sites indicates flexibility in AbrB binding and recognition of three-dimensional DNA structures. Mol Microbiol 19: 145-158.

Yin, S., Daum, R.S., and Boyle-Vavra, S. (2006) VraSR two-component regulatory system and its role in induction of $p b p 2$ and vraSR expression by cell wall antimicrobials in Staphylococcus aureus. Antimicrob Agents Chemother 50: 336-343.

Yoshimura, M., Asai, K., Sadaie, Y., and Yoshikawa, H. (2004) Interaction of Bacillus subtilis extracytoplasmic function (ECF) sigma factors with the N-terminal regions of their potential anti-sigma factors. Microbiology 150: 591-599.

Youngman, P. (1990) Use of transposons and integrational vectors for mutagenesis and construction of gene fusions in Bacillus subtilis. In Molecular biological methods for 
Bacillus. Harwood, C.R. and Cutting, S.M. (eds). Chichester: John Wiley \& sons, pp. 221-266.

Zellmeier, S., Schumann, W., and Wiegert, T. (2006) Involvement of Clp protease activity in modulating the Bacillus subtilis $\sigma^{\mathrm{W}}$ stress response. Molecular Microbiology 61 : 1569-1582.

Zhang, X., Dong, G., and Golden, S.S. (2006) The pseudo-receiver domain of CikA regulates the cyanobacterial circadian input pathway. Mol Microbiol 60: 658-668. 


\section{Chapter 6: Supplementary material}

Table 6.1. Bacillus subtilis strains

\begin{tabular}{|c|c|c|}
\hline Strain & Genotype/Remarks & Reference \\
\hline W168 & laboratory wildtype strain & laboratory strain \\
\hline CU1065 & $\mathrm{W} 168 a t t \mathrm{SPb} 2 \Delta 2 \operatorname{trp} C 2$ & Helmann lab stock \\
\hline HB0920 & CU1065 liaH::kan ${ }^{\mathrm{R}}$ & Mascher et al. 2003 \\
\hline HB0933 & CU1065 liaR::kan ${ }^{\mathrm{R}}$ & Mascher et al. 2003 \\
\hline HB0934 & CU1065 liaGFSR::kan ${ }^{\mathrm{R}}$ & Mascher, unpublished \\
\hline HB0935 & CU1065 liaIH::tet(i) ${ }^{\mathrm{R}}$ & Mascher et al. 2003 \\
\hline HB0938 & CU1065 yhcYZ::cat ${ }^{\mathrm{R}}$ & Mascher, unpublished \\
\hline HB0950 & CU1065 attSPb2 $22:: \operatorname{Tn} 917:: \mathrm{F}\left(\mathrm{P}_{\text {lial-74-cat-lacZ })}\right.$ & Mascher et al. 2004 \\
\hline HB0961 & CU1065 liaI::pMUTIN & Mascher et al. 2003 \\
\hline HB0969 & CU1065 yhcY::pMUTIN, liaH::kan ${ }^{\mathrm{R}}$ & Mascher, unpublished \\
\hline HB0970 & CU1065 yhcZ::pMUTIN, liaH::kan ${ }^{\mathrm{R}}$ & Mascher, unpublished \\
\hline TMB001 & CU1065 liaSR::kan & unpublished \\
\hline TMB002 & CU1065 liaF::kan & Jordan et al. 2006 \\
\hline TMB003 & CU1065 liaG::kan & Jordan et al. 2006 \\
\hline TMB004 & CU1065 liaS::kan & Jordan et al. 2006 \\
\hline TMB005 & CU1065 liaGF::kan & unpublished \\
\hline TMB006 & CU1065 liaFS::kan & Jordan et al. 2006 \\
\hline TMB007 & CU1065 liaGFS::kan & unpublished \\
\hline TMB008 & CU1065 liaI::pMUTIN, liaG::kan & unpublished \\
\hline TMB009 & CU1065 liaI::pMUTIN, liaF::kan & unpublished \\
\hline TMB010 & CU1065 liaI::pMUTIN, liaS::kan & unpublished \\
\hline TMB011 & CU1065 liaI::pMUTIN, liaR::kan & Jordan et al., 2007 \\
\hline TMB012 & CU1065 liaI::pMUTIN, liaGF::kan & unpublished \\
\hline TMB013 & CU1065 liaI::pMUTIN, liaFS::kan & unpublished \\
\hline TMB014 & CU1065 liaI::pMUTIN, liaSR::kan & unpublished \\
\hline TMB015 & CU1065 liaI::pMUTIN, liaGFS::kan & unpublished \\
\hline TMB016 & CU1065 amyE::pTM1 & Jordan et al. 2006 \\
\hline TMB017 & CU1065 amyE::pTM1, liaG::kan & Jordan et al. 2006 \\
\hline TMB018 & CU1065 amyE::pTM1, liaF::kan & Jordan et al. 2006 \\
\hline TMB019 & CU1065 amyE::pTM1, liaS::kan & Jordan et al. 2006 \\
\hline TMB020 & CU1065 amyE::pTM1, liaR::kan & Jordan et al. 2006 \\
\hline TMB021 & CU1065 amyE::pTM1, liaGF::kan & Jordan et al. 2006 \\
\hline TMB022 & CU1065 amyE::pTM1, liaFS::kan & unpublished \\
\hline TMB023 & CU1065 amyE::pTM1, liaGFS::kan & unpublished \\
\hline ТМB024 & CU1065 liaS::kan,thrC::pXTlias & unpublished \\
\hline TMB025 & CU1065 liaF::kan,thrC::pXTliaF1 & unpublished \\
\hline TMB026 & CU1065 liaF::kan,thrC::pXTliaF3 & unpublished \\
\hline TMB027 & HB0950 liaF $\Delta\left(\mathrm{I}_{151}-\mathrm{D}_{235}\right)^{\mathrm{a}}$ & Jordan et al. 2006 \\
\hline TMB028 & $\mathrm{HB} 0950$ liaF $\Delta\left(\mathrm{E}_{126}-\mathrm{D}_{146}\right)^{\mathrm{a}}$ & Jordan et al. 2006 \\
\hline ТМB029 & HB0950 liaF $\Delta\left(\mathrm{S}_{189}-\mathrm{V}_{192}\right)^{\mathrm{a}}$ & Jordan et al. 2006 \\
\hline TMB032 & CU1065 liaSR::kan, bceRS::cat, yvcPQ::mls & unpublished \\
\hline TMB040 & CU1065 yvcB::pMUTIN & unpublished \\
\hline ТМB041 & CU1065 yvkN::pMUTIN & unpublished \\
\hline TMB042 & CU1065 ywkC::pMUTIN & unpublished \\
\hline TMB043 & CU1065 yvrL::pMUTIN & unpublished \\
\hline TMB044 & CU1065 yvrH::pMUTIN & unpublished \\
\hline TMB054 & W168 amyE::pSJ602 & unpublished \\
\hline TMB055 & CU1065 liaI::pMUTIN, yxjM::spec & unpublished \\
\hline TMB056 & CU1065 liaI::pMUTIN, yxjML::spec & unpublished \\
\hline TMB057 & CU1065 liaI::pMUTIN, liaS::kan, yxjM::spec & unpublished \\
\hline TMB058 & CU1065 liaI::pMUTIN, liaS::kan, yxjML::spec & unpublished \\
\hline
\end{tabular}




\begin{tabular}{|c|c|c|}
\hline Strain & Genotype/Remarks & Reference \\
\hline TMB059 & CU1065 liaI::pMUTIN, liaFS::kan, yxjM::spec & unpublished \\
\hline ТМB060 & CU1065 liaI::pMUTIN, liaFS::kan, yxjML::spec & unpublished \\
\hline TMB061 & CU1065 liaI::pMUTIN, liaFS::kan, yhcYZ::cat & unpublished \\
\hline TMB062 & CU1065 liaI::pMUTIN, liaFS::kan, yxjML::spec, yhcYZ::cat & unpublished \\
\hline TMB063 & W168 amyE::pAJ603, yhcZ::MLS & Junker, diploma thesis \\
\hline TMB064 & W168 amyE::pAJ603, yhcZ::MLS, liaR::kan & Junker, diploma thesis \\
\hline TMB065 & W168 amyE::pAJ603, liaHGF::kan & Junker, diploma thesis \\
\hline TMB066 & W168 amyE::pAJ603, liaR::spec & Jordan et al. 2006 \\
\hline TMB067 & W168 amyE::pAJ603, liaF::kan, liaIH::tet (i) & Junker, diploma thesis \\
\hline TMB068 & W168 amyE::pAJ603, liaHGF::kan, yhcZ::MLS & Junker, diploma thesis \\
\hline TMB069 & W168 amyE::pAJ603, liaHGF::kan, liaR::spec & Jordan et al. 2006 \\
\hline TMB070 & W168 amyE::pAJ603, liaH::kan, yhcZ::MLS & Junker, diploma thesis \\
\hline TMB071 & W168 amyE::pAJ603 & Jordan et al. 2006 \\
\hline TMB072 & W168 amyE::pAJ603, liaH::kan & Jordan et al. 2006 \\
\hline TMB073 & W168 amyE::pSJ603 & unpublished \\
\hline TMB074 & W168 amyE::pSJ603, liaF::kan & unpublished \\
\hline TMB075 & W168 amyE::pSJ604 & unpublished \\
\hline TMB076 & W168 amyE::pSJ604, liaF::kan & unpublished \\
\hline TMB077 & W168 amyE::pSJ605 & unpublished \\
\hline TMB078 & W168 amyE::pSJ605, liaIH::tet & unpublished \\
\hline TMB079 & W168 $\sin R::$ spec & Jordan et al., 2007 \\
\hline TMB080 & W168 aprE::kan & Jordan et al., 2007 \\
\hline TMB081 & W168 scoC::tet & Jordan et al., 2007 \\
\hline TMB082 & W168 abrB::kan & Jordan et al., 2007 \\
\hline TMB083 & W168 salA::tet & Jordan et al., 2007 \\
\hline TMB084 & HB0961 $\sin R::$ spec & Jordan et al., 2007 \\
\hline TMB085 & HB0961 aprE::kan & Jordan et al., 2007 \\
\hline TMB086 & HB0961 scoC::tet & Jordan et al., 2007 \\
\hline TMB087 & HB0961 $a b r B:$ kan & Jordan et al., 2007 \\
\hline TMB088 & HB0961 salA::tet & Jordan et al., 2007 \\
\hline TMB089 & W168 amyE::pER601 & Rietkötter, GP II \\
\hline TMB090 & W168 aprE::pER201 & Rietkötter, GP II \\
\hline TMB091 & W168 liaHGF::kan & Junker, diploma thesis \\
\hline TMB092 & W168 liaR::spec & Junker, diploma thesis \\
\hline TMB093 & W168 yhcZ::MLS & Junker, diploma thesis \\
\hline TMB094 & W168 amyE::pAJ603, liaGF::kan & Junker, diploma thesis \\
\hline TMB095 & W168 amyE::pAJ603, liaF::kan & Jordan et al. 2006 \\
\hline TMB096 & W168 amyE::pAJ601, liaHGF::kan & Jordan et al. 2006 \\
\hline TMB097 & W168 amyE::pAJ602, liaHGF::kan & Jordan et al. 2006 \\
\hline TMB098 & W168 amyE::pAJ604, liaHGF::kan & Jordan et al. 2006 \\
\hline TMB099 & W168 amyE::pAJ609, liaHGF::kan & Jordan et al. 2006 \\
\hline TMB100 & W168 amyE::pAJ608, liaHGF::kan & Jordan et al. 2006 \\
\hline TMB101 & W168 amyE::pAJ607, liaHGF::kan & Jordan et al. 2006 \\
\hline TMB102 & W168 amyE::pAJ603, liaHGF::kan & Jordan et al. 2006 \\
\hline TMB103 & W168 amyE::pAJ605, liaHGF::kan & Junker, diploma thesis \\
\hline TMB104 & W168 amyE::pAJ606, liaHGF::kan & Jordan et al. 2006 \\
\hline TMB105 & W168 amyE::pAJ610, liaHGF::kan & Junker, diploma thesis \\
\hline TMB108 & CU1065 amyE::pTM1, liaH::kan & Jordan et al. 2006 \\
\hline TMB109 & CU1065 amyE::pTM1, liaHGF::kan & unpublished \\
\hline TMB110 & W168 amyE::pBD607 (A-77 $\mathrm{T})$ & Dörrbecker, GP II \\
\hline TMB111 & W168 amyE::pBD601 & Jordan et al. 2006 \\
\hline TMB112 & W168 amyE::pBD602 & Jordan et al. 2006 \\
\hline TMB113 & W168 amyE::pBD603 & Jordan et al. 2006 \\
\hline TMB114 & W168 amyE::pBD604 & Jordan et al. 2006 \\
\hline
\end{tabular}




\begin{tabular}{|c|c|c|}
\hline Strain & Genotype/Remarks & Reference \\
\hline TMB115 & W168 amyE::pBD605 & Jordan et al. 2006 \\
\hline TMB116 & W168 amyE::pER602 & Rietkötter, GP II \\
\hline TMB117 & HB0961 $\operatorname{deg} U:: k a n$ & Jordan et al., 2007 \\
\hline TMB118 & HB0961 spo0A::tet & Jordan et al., 2007 \\
\hline TMB119 & TMB090 spo0A::tet & Rietkötter, GP II \\
\hline TMB 120 & TMB090 $\sin R::$ spec & Rietkötter, GP II \\
\hline TMB121 & TMB090 abrB::kan & Rietkötter, GP II \\
\hline TMB122 & TMB089 scoC::tet & Rietkötter, GP II \\
\hline TMB123 & TMB089 abrB::kan & Rietkötter, GP II \\
\hline TMB124 & W168 degU::kan & Jordan et al., 2007 \\
\hline TMB125 & TMB16 liaIH::tet(i) & unpublished \\
\hline TMB126 & TMB116 liaIH::tet(i) & unpublished \\
\hline TMB127 & TMB116 liaH::kan & unpublished \\
\hline TMB128 & W168 amyE::pDH601 & Hoyer, GP II \\
\hline TMB129 & W168 amyE::pDH602 & Hoyer, GP II \\
\hline TMB130 & W168 amyE::pDH603 & Hoyer, GP II \\
\hline TMB131 & W168 amyE::pDH604 & Hoyer, GP II \\
\hline TMB132 & W168 amyE::pDH605 & Jordan et al. 2006 \\
\hline TMB133 & W168 amyE::pBD606 & Jordan et al. 2006 \\
\hline TMB159 & W168 amyE::pAJ601, liaH::kan & Junker, diploma thesis \\
\hline TMB160 & W168 amyE::pAJ602, liaH::kan & Junker, diploma thesis \\
\hline TMB161 & W168 amyE::pAJ604, liaH::kan & Junker, diploma thesis \\
\hline TMB162 & W168 amyE::pAJ605, liaH::kan & Junker, diploma thesis \\
\hline TMB163 & HB0961 $a b h::$ kan & unpublished \\
\hline TMB164 & W168 amyE::pAJ601 & Junker, diploma thesis \\
\hline TMB165 & W168 amyE::pAJ602 & Junker, diploma thesis \\
\hline TMB166 & W168 amyE::pAJ604 & Junker, diploma thesis \\
\hline TMB167 & W168 amyE::pAJ603 & Junker, diploma thesis \\
\hline TMB168 & W168 amyE::pAJ605 & Junker, diploma thesis \\
\hline TMB169 & W168 liaH::kan & Junker, diploma thesis \\
\hline TMB170 & W168 amyE::pSJ608 & unpublished \\
\hline TMB171 & W168 amyE::pSJ610 & unpublished \\
\hline TMB172 & MD300 (degU-hy) small colony liaI::pMUTIN & unpublished \\
\hline TMB173 & MD300 (degU-hy) large colony liaI::pMUTIN & unpublished \\
\hline TMB174 & W168 ackA::mls & unpublished \\
\hline TMB175 & W168 amyE::pAJ611 & Junker, diploma thesis \\
\hline TMB176 & W168 amyE::pAJ610 & Junker, diploma thesis \\
\hline TMB177 & W168 amyE::pAJ609 & Junker, diploma thesis \\
\hline TMB178 & W168 amyE::pAJ608 & Junker, diploma thesis \\
\hline TMB179 & W168 amyE::pAJ607 & Junker, diploma thesis \\
\hline TMB180 & W168 amyE::pAJ606 & Junker, diploma thesis \\
\hline TMB181 & W168 liaSR clean deletion & unpublished \\
\hline TMB183 & TMB028 thrC::pSJ701 & Jordan et al. 2006 \\
\hline TMB184 & TMB029 thrC::pSJ701 & Jordan et al. 2006 \\
\hline TMB185 & TMB009 amyE::pSJ901 & unpublished \\
\hline TMB186 & W168 pta::tet & unpublished \\
\hline TMB187 & TMB016 pta::tet & unpublished \\
\hline TMB188 & TMB019 pta::tet & unpublished \\
\hline TMB189 & TMB022 pta::tet & unpublished \\
\hline TMB190 & TMB016 ackA::mls & unpublished \\
\hline TMB191 & TMB019 ackA::mls & unpublished \\
\hline TMB192 & TMB022 ackA::mls & unpublished \\
\hline TMB193 & W168 pta::tet, ackA::mls & unpublished \\
\hline TMB194 & TMB016 pta::tet, ackA::mls & unpublished \\
\hline
\end{tabular}




\begin{tabular}{|c|c|c|}
\hline Strain & Genotype/Remarks & Reference \\
\hline TMB195 & TMB019 pta::tet, ackA::mls & unpublished \\
\hline TMB196 & TMB022 pta::tet, ackA::mls & unpublished \\
\hline TMB197 & TMB170 liaF::kan & unpublished \\
\hline TMB198 & TMB170 yhcZ::mls & unpublished \\
\hline TMB199 & HB0961 liaFS::kan, pta::tet & unpublished \\
\hline TMB200 & W168 pta::tet, liaS::kan & unpublished \\
\hline TMB201 & W168 pta::tet, liaFS::kan & unpublished \\
\hline TMB202 & W168 ackA::mls, liaS::kan & unpublished \\
\hline TMB203 & W168 ackA::mls, liaFS::kan & unpublished \\
\hline TMB204 & TMB181 amyE::pTM1 & unpublished \\
\hline TMB205 & W168 spo0A::tet & Jordan et al., 2007 \\
\hline TMB206 & TMB204 thrC::pSJ702 & unpublished \\
\hline TMB208 & W168 abrB::kan, spo0A::tet & unpublished \\
\hline TMB209 & W168 liaI::pMUTIN, abrB::kan, spo0A::tet & Jordan et al., 2007 \\
\hline TMB210 & W168 liaR::spec, abrB::kan & unpublished \\
\hline TMB212 & W168 liaR::spec, abrB::kan, liaI::pMUTIN & unpublished \\
\hline TMB213 & W168 liaS clean deletion & Jordan et al., 2007 \\
\hline TMB214 & W168 liaFS clean deletion & Martinec, GP II \\
\hline TMB215 & W168 liaS clean deletion, liaI::pMUTIN & Jordan et al., 2007 \\
\hline TMB216 & W168 liaS clean deletion, amyE::pTM1 & Martinec, GP II \\
\hline TMB217 & W168 liaFS clean deletion, lial::pMUTIN & Martinec, GP II \\
\hline TMB218 & W168 liaFS clean deletion, amyE::pTM1 & Martinec, GP II \\
\hline TMB219 & W168 amyE::pSK601(wapA-promoter) & unpublished \\
\hline TMB220 & TMB001 amyE::pSK601 & unpublished \\
\hline TMB221 & TMB002 amyE::pSK601 & unpublished \\
\hline TMB222 & W168 amyE::pSK602(ydhE-promoter) & unpublished \\
\hline TMB223 & TMB001 amyE::pSK602 & unpublished \\
\hline TMB224 & TMB002 amyE::pSK602 & unpublished \\
\hline TMB229 & W168 liaS clean deletion, lial::pMUTIN, thrC::pSJ702 & unpublished \\
\hline TMB230 & W168 liaS clean deletion, amyE::pTM1, thrC::pSJ702 & unpublished \\
\hline TMB231 & W168 liaI::pMUTIN, liaS::kan, LiaR $\mathrm{D}_{54} \mathrm{~A}$ & Schrecke, GP II \\
\hline TMB232 & W168 amyE::pTM1, liaS::kan, LiaR D $\mathrm{D}_{54} \mathrm{~A}$ & Schrecke, GP II \\
\hline TMB233 & W168 liaS clean deletion, liaI::pMUTIN, thrC::pXT-liaS & Schrecke, GP II \\
\hline TMB234 & W168 liaS clean deletion, liaI::pMUTIN, thrC::pXT-liaS (SD) & Schrecke, GP II \\
\hline TMB235 & W168 liaS clean deletion, amyE::pTM1, thrC::pXT-liaS & Schrecke, GP II \\
\hline TMB236 & W168 liaS clean deletion, amyE::pTM1, thrC::pXT-liaS (SD) & Schrecke, GP II \\
\hline TMB237 & W168 liaFS clean del., liaI::pMUTIN, thrC::pXT-liaFS & Schrecke, GP II \\
\hline TMB238 & W168 liaFS clean del., liaI::pMUTIN, thrC::pXT-liaFS (SD) & Schrecke, GP II \\
\hline TMB239 & W168 amyE::pSJ611-lacZ & unpublished \\
\hline TMB240 & W168 amyE::pSJ612-lacZ & unpublished \\
\hline TMB243 & HB0961 liaHGF::kan & unpublished \\
\hline TMB244 & HB0961 liaGFSR::kan & unpublished \\
\hline TMB245 & TMB16 liaHGF::kan & unpublished \\
\hline TMB246 & TMB16 liaGFSR::kan & unpublished \\
\hline TMB247 & W168 liaS::kan, $\operatorname{LiaR}\left(\mathrm{D}_{54} \mathrm{~A}\right)$ & Schrecke, GP II \\
\hline TMB248 & TMB215 (W168 liaS clean deletion, liaI::pMUTIN) thrC::pXT & Schrecke, GP II \\
\hline TMB249 & TMB216 (W168 liaS clean deletion, amyE::pTM1), thrC::pXT & Schrecke, GP II \\
\hline TMB250 & TMB217 (W168 liaFS clean deletion, lial::pMUTIN), thrC::pXT & Schrecke, GP II \\
\hline TMB251 & TMB218 (W168 liaFS clean deletion, amyE::pTM1), thrC::pXT & Schrecke, GP II \\
\hline TMB252 & TMB010 (HB0961 liaS::kan) pta::tet & Schrecke, GP II \\
\hline TMB254 & TMB027 thrC::pSJ704 & unpublished \\
\hline TMB255 & TMB027 thrC::pSJ705 & unpublished \\
\hline TMB256 & TMB027 thrC::pSJ706 & unpublished \\
\hline TMB257 & TMB027 thrC::pSJ709 & unpublished \\
\hline
\end{tabular}




\begin{tabular}{|c|c|c|}
\hline Strain & Genotype/Remarks & Reference \\
\hline TMB258 & TMB027 thrC::pSJ710 & unpublished \\
\hline TMB259 & TMB027 thrC::pSJ711 & unpublished \\
\hline TMB260 & TMB027 thrC::pSJ712 & unpublished \\
\hline TMB261 & TMB027 thrC::pSJ714 & unpublished \\
\hline TMB262 & TMB027 thrC::pSJ715 & unpublished \\
\hline TMB263 & W168 thrC::pXT & unpublished \\
\hline TMB264 & $\mathrm{W} 168$ thrC::pSJ703 & unpublished \\
\hline TMB265 & W168 thrC::pSJ707 & unpublished \\
\hline TMB266 & W168 thrC::pSJ708 & unpublished \\
\hline TMB267 & $\mathrm{W} 168$ thrC::pSJ713 & unpublished \\
\hline TMB268 & TMB018 (liaF::kan, amyE::pTM1) thrC::pSJ701 & unpublished \\
\hline TMB269 & $\mathrm{W} 168$ thrC::pSJ706 & unpublished \\
\hline TMB272 & $\mathrm{W} 168$ amyE:: $\mathrm{P}_{y d h E 2}-l a c Z$ & unpublished \\
\hline TMB273 & $\mathrm{W} 168$ amyE:: $\mathrm{P}_{y d h E 3}-l a c Z$ & unpublished \\
\hline TMB274 & $\mathrm{W} 168$ amyE $:: \mathrm{P}_{y d h E 2}-l a c Z$, liaF $:$ kan & unpublished \\
\hline TMB275 & $\mathrm{W} 168$ amyE $:: \mathrm{P}_{y d h E 3}-l a c Z$, liaF $:: \mathrm{kan}$ & unpublished \\
\hline TMB276 & W168 amyE::pER501 ( $\mathrm{P}_{\text {liaG }}$ nativ to ATG of liaF) & Rietkötter, unpublished \\
\hline TMB277 & W168 amyE::pER502 ( $\mathrm{P}_{\text {liaG }}$ optimal -10 to ATG of liaF) & Rietkötter, unpublished \\
\hline TMB278 & W168 amyE::pER503 ( $\mathrm{P}_{\text {liaG }}$ optimal -10 und -35 bis ATG von liaF) & Rietkötter, unpublished \\
\hline TMB279 & $\mathrm{W} 168$ amyE::pER603 ( $\left.\mathrm{P}_{b c e A}-l a c Z\right)$ & Rietkötter, unpublished \\
\hline TMB280 & TMB035 (bceAB::kan) amyE:::pER603 ( $\left.\mathrm{P}_{b c e A}-l a c Z\right)$ & Rietkötter, unpublished \\
\hline TMB281 & W168 amyE::pER504 ( $\mathrm{P}_{\text {liaG }}$ optimal -10 and -35 to ATG of LiaS) & Rietkötter, unpublished \\
\hline TMB282 & W168 amyE::pER505 ( $\mathrm{P}_{\text {liaG }}$ optimal -10 and -35 to ATG of LiaR) & Rietkötter, unpublished \\
\hline TMB283 & TMB216 thrC::pSJ722 & unpublished \\
\hline TMB284 & TMB216 thrC::pSJ723 & unpublished \\
\hline TMB285 & TMB216 thrC::pSJ725 & unpublished \\
\hline TMB286 & TMB216 thrC::pSJ726 & unpublished \\
\hline TMB304 & TMB216 thrC::pSJ721 & unpublished \\
\hline TMB305 & TMB215 thrC::pSJ719 & unpublished \\
\hline TMB306 & TMB215 thrC::pSJ720 & unpublished \\
\hline TMB307 & TMB215 thrC::pSJ721 & unpublished \\
\hline TMB308 & TMB215 thrC::pSJ722 & unpublished \\
\hline TMB309 & TMB215 thrC::pSJ723 & unpublished \\
\hline TMB310 & TMB215 thrC::pSJ724 & unpublished \\
\hline TMB311 & TMB215 thrC::pSJ725 & unpublished \\
\hline TMB312 & TMB215 thrC::pSJ726 & unpublished \\
\hline TMB313 & TMB215 thrC::pSJ727 & unpublished \\
\hline TMB314 & $\mathrm{W} 168$ thrC::pSJ716 & unpublished \\
\hline TMB315 & W168 thrC::pSJ717 & unpublished \\
\hline TMB318 & W168 amyE::pSJ4001 & unpublished \\
\hline TMB319 & W168 amyE::pSJ4002 & unpublished \\
\hline TMB320 & W168 amyE::pSJ4003 & unpublished \\
\hline TMB321 & W168 amyE::pSJ5402 & unpublished \\
\hline TMB322 & W168 amyE::pSJ5401 & unpublished \\
\hline TMB323 & W168 amyE::pSJ5404 & unpublished \\
\hline TMB324 & W168 amyE::pSJ5403 $\left(\operatorname{LiaF~}_{158} \mathrm{~V}\right)$ & unpublished \\
\hline TMB329 & W168 liaF clean deletion & unpublished \\
\hline TMB330 & TMB215 abrB::kan & Jordan et al., 2007 \\
\hline TMB331 & TMB329 amyE::pTM1 & unpublished \\
\hline TMB332 & TMB331 thrC::pXT & unpublished \\
\hline TMB333 & TMB331 thrC::pSJ701 & unpublished \\
\hline TMB334 & TMB331 thrC::pSJ704 & unpublished \\
\hline TMB335 & TMB331 thrC::pSJ705 & unpublished \\
\hline TMB336 & TMB331 thrC::pSJ706 & unpublished \\
\hline
\end{tabular}




\begin{tabular}{|c|c|c|}
\hline Strain & Genotype/Remarks & Reference \\
\hline TMB337 & TMB331 thrC::pSJ709 & unpublished \\
\hline TMB338 & TMB331 thrC::pSJ710 & unpublished \\
\hline TMB339 & TMB331 thrC::pSJ711 & unpublished \\
\hline TMB340 & TMB331 thrC::pSJ712 & unpublished \\
\hline TMB341 & TMB331 thrC::pSJ714 & unpublished \\
\hline TMB342 & TMB331 thrC::pSJ715 & unpublished \\
\hline TMB343 & TMB331 thrC::pSJ703 & unpublished \\
\hline TMB344 & TMB331 thrC::pSJ707 & unpublished \\
\hline TMB345 & TMB331 thrC::pSJ708 & unpublished \\
\hline TMB346 & TMB331 thrC::pSJ713 & unpublished \\
\hline TMB347 & TMB331 thrC::pSJ716 & unpublished \\
\hline TMB348 & TMB331 thrC::pSJ717 & unpublished \\
\hline TMB349 & TMB331 thrC::pSJ718 & unpublished \\
\hline TMB350 & TMB329 liaI::pMUTIN & unpublished \\
\hline TMB351 & TMB350 thrC::pXT & unpublished \\
\hline TMB352 & TMB350 thrC::pSJ701 & unpublished \\
\hline TMB353 & TMB350 thrC::pSJ704 & unpublished \\
\hline TMB354 & TMB350 thrC::pSJ705 & unpublished \\
\hline TMB355 & TMB350 thrC::pSJ706 & unpublished \\
\hline TMB356 & TMB350 thrC::pSJ709 & unpublished \\
\hline TMB357 & TMB350 thrC::pSJ710 & unpublished \\
\hline TMB358 & TMB350 thrC::pSJ711 & unpublished \\
\hline TMB359 & TMB350 thrC::pSJ712 & unpublished \\
\hline TMB360 & TMB350 thrC::pSJ714 & unpublished \\
\hline TMB361 & TMB350 thrC::pSJ715 & unpublished \\
\hline TMB362 & TMB350 thrC::pSJ703 & unpublished \\
\hline TMB363 & TMB350 thrC::pSJ707 & unpublished \\
\hline TMB364 & TMB350 thrC::pSJ708 & unpublished \\
\hline TMB365 & TMB350 thrC::pSJ713 & unpublished \\
\hline TMB366 & TMB350 thrC::pSJ716 & unpublished \\
\hline TMB367 & TMB350 thrC::pSJ717 & unpublished \\
\hline TMB368 & TMB350 thrC::pSJ718 & unpublished \\
\hline TMB369 & W168 liaH::pSJ6401 & unpublished \\
\hline TMB370 & W168 liaI::pMUTIN & unpublished \\
\hline TMB396 & W168 amyE::pSJ2901 & unpublished \\
\hline TMB397 & W168 amyE::pSJ2902 & unpublished \\
\hline TMB398 & W168 amyE::pSJ2903 & unpublished \\
\hline TMB399 & W168 amyE::pSJ2904 & unpublished \\
\hline TMB402 & TMB350 amyE::pSJ5403 & unpublished \\
\hline TMB403 & TMB215 amyE::pSJ5404 & unpublished \\
\hline TMB404 & TMB350 amyE::pSJ2902 & unpublished \\
\hline TMB405 & TMB215 amyE::pSJ2903 & unpublished \\
\hline TMB406 & TMB370 amyE::pSJ5404 & unpublished \\
\hline TMB407 & TMB011 amyE::pSJ2904-liaR & unpublished \\
\hline TMB408 & $\mathrm{W} 168 \mathrm{P}_{\text {lial }}:: \mathrm{pSJ} 5101$ & unpublished \\
\hline
\end{tabular}

resistance cassettes: kan: kanamycin, cat: chloramphenicol, mls: erythromycin/lincomycin, spec: spectinomycin, tet $=$ tetracyclin. Positions of cloned fragments are given relative to the start codon.

${ }^{a}$ For reasons of clarity, the effects of in-frame deletions in lia $F$ are given at the level of LiaF protein.

Table 6.2. Escherichia coli strains

\begin{tabular}{|c|c|c|}
\hline Strain & Genotype/Remarks & Reference \\
\hline DH5 $\alpha$ & 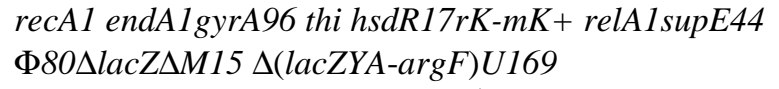 & Sambrook and Russel, 2001 \\
\hline $71 / 18$ & supE thi $\Delta($ lac-proAB $) \mathrm{F}^{\prime}\left[\operatorname{ProAB}^{+}\right.$lacI ${ }^{q}$ lac $\left.\mathrm{Z} \Delta \mathrm{M} 15\right]$ & Sambrook and Russel, 2001 \\
\hline
\end{tabular}




\begin{tabular}{lll}
\hline Strain & Genotype/Remarks & Reference \\
\hline CC118 & $\begin{array}{l}\Delta(\text { ara-leu }) 7697 \Delta \text { lacX74 } \triangle \text { phoA20 galE galK thi rpsE rpoB } \\
\text { TrgE(am) recAl }\end{array}$ & Manoil and Beckwith, 1985 \\
TME011 & CC118 with pSJ401 & unpublished \\
TME012 & CC118 with pSJ402 & unpublished \\
TME013 & CC118 with pSJ403 & unpublished \\
TME014 & CC118 with pSJ404 & unpublished \\
TME015 & CC118 with pSJ405 & unpublished \\
TME016 & CC118 with pSJ406 & unpublished \\
TME017 & CC118 with pSJ407 & unpublished \\
TME018 & CC118 with pSJ408 & unpublished \\
TME019 & CC118 with pSJ409 & \\
\hline
\end{tabular}

Table 6.3. Vectors and plasmids

\begin{tabular}{|c|c|c|}
\hline Vector & Description & Reference \\
\hline$\overline{\mathrm{pAC6}}$ & bla, lacZ, cat; lacZ fusion vector, integrates at $a m y E$, & Stülke et al., 1997 \\
\hline pHA-4 & $\begin{array}{l}\text { araC, bla, phoA, pNG backbone; C-term. PhoA fusion for membrane } \\
\text { topology analysis in } E \text {. coli }\end{array}$ & $\begin{array}{l}\text { Rapp et al. 2004, Daley } \\
\text { et al. } 2005\end{array}$ \\
\hline pET-16b & $\begin{array}{l}\text { PT7, His(10)-tag, tT7, lacI, ori(pBR322), bla; protein overexpression } \\
\text { vector }\end{array}$ & $\begin{array}{l}\text { pET system manual, 10th } \\
\text { ed. }\end{array}$ \\
\hline pGFPe & kan, GFP, His-tag, pET128 backbone & $\begin{array}{l}\text { Rapp et al. 2004, Daley } \\
\text { et al. } 2005\end{array}$ \\
\hline pJPM122 & MCS, cat-lacZ, bla, neo, ori; integrates at attSPb & Slack et al. 1993 \\
\hline pMAD & erm, ori(pE194-Ts), MCS-P clpB $^{-b g a B}$, ori(pBR322), bla & Arnaud et al. 2004 \\
\hline pSG1151 & bla cat gfpmut 1 & Lewis and Marston, 1999 \\
\hline pSG1154 & bla amyE3'spc $\mathrm{P}_{x y l^{\prime}}$ 'gfpmut1 amyE5'; C-terminal fusion vector & Lewis and Marston, 1999 \\
\hline pSG1164 & bla cat $\mathrm{P}_{x y l}$-gfpmut1 & Lewis and Marston, 1999 \\
\hline pSG1729 & bla amyE3'spc $\mathrm{P}_{x y l^{\prime}}$ 'gfpmutl amyE5'; N-terminal fusion vector & Lewis and Marston, 1999 \\
\hline pSH4 & pSG1154 with mutation in $g f p$ & Halbedel, unpublished \\
\hline pSH5 & pSG1164 with mutation in $g f p$ & Halbedel, unpublished \\
\hline $\mathrm{pXT}$ & $\begin{array}{l}\text { vector for xylose-inducible gene expression, integrates at thrC, } \\
\text { pDG1782-derivative, spectinomycin resistance }\end{array}$ & Derre et al., 2000 \\
\hline Plasmid & Description & Reference \\
\hline pAJ601 & $\mathrm{P}_{\text {yhcY-29 }}$ EcoRI/BamHI (primer 165/172) into pAC6 & Jordan et al., 2006 \\
\hline pAJ602 & $\mathrm{P}_{y h c Y-46} E c o \mathrm{RI} /$ BamHI (primer 165/171) into pAC6 & Jordan et al., 2006 \\
\hline pAJ603 & $\mathrm{P}_{\text {yhcY-87 }}$ EcoRI/BamHI (primer 165/168) into pAC6 & Jordan et al., 2006 \\
\hline pAJ604 & $\mathrm{P}_{\text {yhcY-55 }}$ EcoRI/BamHI (primer 165/170) into pAC6 & Jordan et al., 2006 \\
\hline pAJ605 & $\mathrm{P}_{y h c Y-99}$ EcoRI/BamHI (primer 165/167) into pAC6 & Jordan et al., 2006 \\
\hline pAJ606 & $\mathrm{P}_{y h c Y-202}$ EcoRI/BamHI (primer 165/166) into pAC6 & Jordan et al., 2006 \\
\hline pAJ607 & $\mathrm{P}_{y h c Y-80}$ EcoRI/BamHI (primer 165/169) into pAC6 & Jordan et al., 2006 \\
\hline pAJ608 & $\mathrm{P}_{y h c Y-75}$ EcoRI/BamHI (primer 165/259) into pAC6 & Jordan et al., 2006 \\
\hline pAJ609 & $\mathrm{P}_{y h c Y-65}$ EcoRI/BamHI (primer 165/260) into pAC6 & Jordan et al., 2006 \\
\hline pBD601 & liaI (-102 - 72) XhoI/BamHI (primer 231/100) into pAC6 & Jordan et al., 2006 \\
\hline pBD602 & liaI (-102 - 72) XhoI/BamHI (primer 232/100) into pAC6 & Jordan et al., 2006 \\
\hline pBD603 & liaI (-102 - 72) XhoI/BamHI (primer 265/100) into pAC6 & Jordan et al., 2006 \\
\hline pBD604 & liaI (-102 - 72) XhoI/BamHI (primer 266/100) into pAC6 & Jordan et al., 2006 \\
\hline pBD605 & liaI (-102 - 72) XhoI/BamHI (primer 267/100) into pAC6 & Jordan et al., 2006 \\
\hline pBD606 & liaI (-102 - 72) XhoI/BamHI (primer 268/100) into pAC6 & Jordan et al., 2006 \\
\hline pDH601 & liaF (-21 - -157) EcoRI/BamHI (primer 204/218) into pAC6 & Hoyer, GP II \\
\hline pDH602 & liaF (-21 - -250) EcoRI/BamHI (primer 204/219) into pAC6 & Hoyer, GP II \\
\hline pDH603 & liaF (-21 - -336) EcoRI/BamHI (primer 204/220) into pAC6 & Hoyer, GP II \\
\hline pDH604 & liaF (-21 - -546) EcoRI/BamHI (primer 204/221) into pAC6 & Hoyer, GP II \\
\hline pDH605 & liaF (-21 - -824) EcoRI/BamHI (primer 204/222) into pAC6 & Jordan et al., 2006 \\
\hline pKS701 & liaS (-24 - end) Bam HI/HindIII (primer 454/46) into pXT & Schrecke, GP II \\
\hline pKS702 & liaS (-24 - end) BamHI/HindIII (primer 495/46) into pXT & Schrecke, GP II \\
\hline pKS703 & liaFS (-21 - end) XhoI/HindIII (primer 211/46) into pXT & Schrecke, GP II \\
\hline
\end{tabular}




\begin{tabular}{|c|c|c|}
\hline Plasmid & Description & Reference \\
\hline pMM101 & $\begin{array}{l}\text { liaS clean deletion BamHI/EcoRI (fused fragments primer 246/462, } \\
\text { 461/249) into pMAD }\end{array}$ & Martinec, GP II \\
\hline pMM102 & $\begin{array}{l}\text { liaFS clean deletion BamHI/EcoRI (fused fragments primer } 457 / 463 \text {, } \\
461 / 249 \text { ) into pMAD }\end{array}$ & Martinec, GP II \\
\hline pSJ101 & $\begin{array}{l}\text { liaSR clean deletion BamHI/EcoRI (fused fragments primer 246/248, } \\
247 / 249 \text { ) into pMAD }\end{array}$ & unpublished \\
\hline pSJ102 & $\begin{array}{l}\text { liaF clean deletion BamHI/NcoI/XhoI (primer 457/574, 575/458) into } \\
\text { pMAD }\end{array}$ & unpublished \\
\hline pSJ301 & liaF (-21 - 135) XhoI/BamHI (primer 211/212) into pGFPe & unpublished \\
\hline pSJ302 & liaF (-21 - 303) XhoI/BamHI (primer 211/213)) into pGFPe & unpublished \\
\hline pSJ303 & liaI (-22 - 339) XhoI/BamHI (primer 214/215) into pGFPe & unpublished \\
\hline pSJ304 & liaS(-24 - 213) XhoI/BamHI (primer 216/217) into pGFPe & unpublished \\
\hline pSJ305 & lias (-24 - 123) XhoI/BamHI (primer 216/299) into pGFPe & unpublished \\
\hline pSJ306 & liaG (-25 - 183) XhoI/BamHI (primer 297/330) into pGFPe & unpublished \\
\hline pSJ401 & liaF (-21 - 135) XhoI/BamHI (primer 211/212) into pHA-4 & unpublished \\
\hline pSJ402 & liaF (-21 - 303) XhoI/BamHI (primer 211/213) into pHA-4 & unpublished \\
\hline pSJ403 & liaI (-22 - 339) XhoI/BamHI (primer 214/215) into pHA-4 & unpublished \\
\hline pSJ404 & liaS (-24 - 213) XhoI/BamHI (primer 216/217) into pHA-4 & unpublished \\
\hline pSJ405 & liaS (-24 - 123) XhoI/BamHI (primer 216/299) into pHA-4 & unpublished \\
\hline pSJ406 & liaG (-25 - 183) XhoI/BamHI (primer 297/330) into pHA-4 & unpublished \\
\hline pSJ407 & liaF (-1 - 135) XhoI/BamHI (primer 370/212) into pHA-4 & unpublished \\
\hline pSJ408 & liaF (-1 - 303) XhoI/BamHI (primer 370/212) into pHA-4 & unpublished \\
\hline pSJ409 & liaF (-1 - 195) XhoI/BamHI (primer 370/371) into pHA-4 & unpublished \\
\hline pSJ601 & liaG (-68 - 914) EcoRI/BamHI (primer 204/205) into pAC6 & Jordan et al., 2006 \\
\hline pSJ602 & liaF (-21 - 727) EcoRI/BamHI (primer 206/132) into pAC6 & unpublished \\
\hline pSJ603 & $\mathrm{P}_{\text {yozJ }}(-306$ - 86) EcoRI/BamHI (primer 241/242) into pAC6 & unpublished \\
\hline pSJ604 & $\mathrm{P}_{\text {rapK }}(-1291$ - -957) EcoRI/BamHI (primer 243/244) into pAC6 & unpublished \\
\hline pSJ605 & $\mathrm{P}_{\text {rapK }}(-1291$ - -235) EcoRI/BamHI (primer 243/245) into pAC6 & unpublished \\
\hline pSJ606 & liaG (-68 - 105) EcoRI/BamHI (primer 204/295) into pAC6 & unpublished \\
\hline pSJ607 & liaG (-68 - 3) EcoRI/BamHI (primer 204/296) into pAC6 & Jordan et al., 2006 \\
\hline pSJ608 & $\mathrm{P}_{\text {gиас }}(-359-43) E c o \mathrm{RI} /$ Bam HI (primer 368/369) into pAC6 & unpublished \\
\hline pSJ609 & $\mathrm{P}_{\text {yuzG }}(-321-81) E c o \mathrm{RI} /$ BamHI (primer 366/367) into pAC6 & unpublished \\
\hline pSJ610 & $\mathrm{P}_{\text {lial }} /$ liaI (-109 - end) EcoRI/BamHI (primer 099/192) into pAC6 & unpublished \\
\hline pSJ611 & $\mathrm{P}_{y d h E}(-170$ - 128) EcoRI/BamHI (primer 480/525) into pAC6 & unpublished \\
\hline pSJ612 & $\mathrm{P}_{y d h E}(-170-128) E c o \mathrm{RI} /$ BamHI (primer 480/526) into pAC6 & unpublished \\
\hline pSJ701 & liaF (-22 - end) HindIII/EcoRI (primer 35/36) into pXT & unpublished \\
\hline pSJ702 & liaS (-22 - end) BamHI/HindIII (primer 44/46) into pXT & unpublished \\
\hline pSJ703 & liaF $\mathrm{G}_{128} \mathrm{~A}^{\mathrm{a}}(-22$ - end) HindIII/EcoRI (primer $35 / 36$ and 500 ) into pXT & unpublished \\
\hline pSJ704 & liaF $\mathrm{G}_{149} \mathrm{~A}^{\mathrm{a}}(-22$ - end) HindIII/EcoRI (primer $35 / 36$ and 501) into pXT & unpublished \\
\hline pSJ705 & liaF $\mathrm{G}_{174} \mathrm{~A}^{\mathrm{a}}$ (-22 - end) HindIII/EcoRI (primer $35 / 36$ and 502) into $\mathrm{pXT}$ & unpublished \\
\hline pSJ706 & liaF $\mathrm{G}_{195} \mathrm{~A}^{\mathrm{a}}$ (-22 - end) HindIII/EcoRI (primer $35 / 36$ and 503) into $\mathrm{pXT}$ & unpublished \\
\hline pSJ707 & liaF $\mathrm{G}_{234} \mathrm{~A}^{\mathrm{a}}$ (-22 - end) HindIII/EcoRI (primer 35/494) into pXT & unpublished \\
\hline pSJ708 & liaF $\mathrm{D}_{141} \mathrm{~A}^{\mathrm{a}}$ (-22 - end) HindIII/EcoRI (primer $35 / 36$ and 504) into pXT & unpublished \\
\hline pSJ709 & liaF $\mathrm{D}_{154} \mathrm{~A}^{\mathrm{a}}$ (-22 - end) HindIII/EcoRI (primer $35 / 36$ and 505) into pXT & unpublished \\
\hline pSJ710 & liaF $\mathrm{D}_{183} \mathrm{~A}^{\mathrm{a}}$ (-22 - end) HindIII/EcoRI (primer $35 / 36$ and 506) into pXT & unpublished \\
\hline pSJ711 & liaF $\mathrm{P}_{181} \mathrm{~A}^{\mathrm{a}}(-22$ - end) HindIII/EcoRI (primer $35 / 36$ and 507) into $\mathrm{pXT}$ & unpublished \\
\hline pSJ712 & liaF $\Delta \mathrm{TM} 1-4$ (-22 - end) HindIII/EcoRI (primer 489/36) into pXT & unpublished \\
\hline pSJ713 & $\begin{array}{l}\text { liaF } \Delta \mathrm{TM} 2 \text { and } 3 \text { (-22 - end) HindIII/EcoRI (fused fragments primer } \\
35 / 490,36 / 491 \text { ) into pXT }\end{array}$ & unpublished \\
\hline pSJ714 & liaF stop after $\mathrm{G}_{195}{ }^{\mathrm{a}}$ (-22 - end) HindIII/EcoRI (primer 35/492) into pXT & unpublished \\
\hline pSJ715 & liaF stop after $\mathrm{E}_{220}{ }^{\mathrm{a}}(-22$ - end) HindIII/EcoRI (primer 35/493) into pXT & unpublished \\
\hline pSJ716 & liaF $\mathrm{D}_{235} \mathrm{~A}^{\mathrm{a}}(-22$ - end) HindIII/EcoRI (primer 35/557) into pXT & unpublished \\
\hline pSJ717 & liaF $\mathrm{D}_{237} \mathrm{~A}^{\mathrm{a}}$ (-22 - end) HindIII/EcoRI (primer 35/556) into $\mathrm{pXT}$ & unpublished \\
\hline pSJ718 & $\begin{array}{l}\text { liaF TM from YxjM HindIII/EcoRI (fused fragments primer } 568 / 569 \text {, } \\
\text { 36/570) into pXT }\end{array}$ & unpublished \\
\hline
\end{tabular}




\begin{tabular}{|c|c|c|}
\hline Plasmid & Description & Reference \\
\hline pSJ719 & cyt. part of liaS (216 -end) BamHI/HindIII (primer 46/529) into pXT & unpublished \\
\hline pSJ720 & $\begin{array}{l}\text { cytoplasmic part of liaS with optimal SD (216 -end) BamHI/HindIII } \\
\text { (primer 46/530) into pXT }\end{array}$ & unpublished \\
\hline pSJ721 & $\begin{array}{l}\text { liaS } \mathrm{D}_{104}-\mathrm{E}_{106} \rightarrow \mathrm{A}_{104-106}{ }^{\mathrm{a}} \text { (-24 - end) BamHI/HindIII (fused fragments } \\
\text { primer } 454 / 536,46 / 534 \text { ) into pXT }\end{array}$ & unpublished \\
\hline pSJ722 & $\begin{array}{l}\text { liaS } \Delta \mathrm{D}_{104}-\mathrm{E}_{106} \text { a }(-24 \text { - end) Bam } \mathrm{HI} / \text { HindIII (fused fragments primer } \\
454 / 537,46 / 535) \text { into pXT }\end{array}$ & unpublished \\
\hline pSJ723 & $\begin{array}{l}\text { liaS } \mathrm{Q}_{202}-\mathrm{R}_{206} \rightarrow \mathrm{A}_{202-206}{ }^{a} \text { (-24 - end) Bam } \mathrm{HI} / \text { HindIII (fused fragments } \\
\text { primer } 454 / 540,46 / 538 \text { ) into pXT }\end{array}$ & unpublished \\
\hline pSJ724 & $\begin{array}{l}\text { liaS } \Delta \mathrm{Q}_{202}-\mathrm{A}_{207}{ }^{\mathrm{a}} \text { (-24 - end) Bam HI/HindIII (fused fragments primer } \\
454 / 541,46 / 539 \text { ) into pXT }\end{array}$ & unpublished \\
\hline pSJ725 & $\begin{array}{l}\text { liaS } \mathrm{H}_{211}-\mathrm{P}_{214} \rightarrow \mathrm{A}_{211-214}{ }^{\mathrm{a}}(-24 \text { - end) BamHI/HindIII (fused fragments } \\
\text { primer } 454 / 544,46 / 542) \text { into } \mathrm{pXT}\end{array}$ & unpublished \\
\hline pSJ726 & $\begin{array}{l}\text { liaS } \Delta \mathrm{H}_{211}-\mathrm{P}_{214} \text { a }(-24 \text { - end) Bam } \mathrm{HI} / \text { HindIII (fused fragments primer } \\
454 / 545,46 / 543 \text { ) into } \mathrm{pXT}\end{array}$ & unpublished \\
\hline pSJ727 & $\begin{array}{l}\text { lias TM from YxdK BamHI/HindIII (fused fragments primer 571/572, } \\
\text { 573/46) into pXT }\end{array}$ & unpublished \\
\hline pSJ1601 & liaI (256 - end) NdeI/BamHI (primer 191/192) & unpublished \\
\hline pSJ1602 & liaF (256 - end) NdeI/BamHI (primer 132/193) & unpublished \\
\hline pSJ2901 & liaI (1 - end) ClaI/XhoI (primer 738/743) & unpublished \\
\hline pSJ2902 & liaF (1 - end) HindIII/XhoI (primer 740/729) & unpublished \\
\hline pSJ2903 & liaS (1 - end) HindIII/XhoI (primer 741/731) & unpublished \\
\hline pSJ2904 & liaR (1 - end) HindIII/XhoI (primer 742/733) & unpublished \\
\hline pSJ5101 & $P_{\text {lial }}(-109--7)$ EcoRI/PstI (primer 99/627) into pSG1151 & unpublished \\
\hline pSJ5401 & liaI (4 - end) KpnI/XhoI (primer 558/559) into pSG1154 & unpublished \\
\hline pSJ5402 & liaH (4 - end) KpnI/XhoI (primer 560/561) into pSG1154 & unpublished \\
\hline pSJ5403 & liaF (4 - end) KpnI/XhoI (primer 564/565) into pSG1154 & unpublished \\
\hline pSJ5404 & liaS (4 - end) KpnI/XhoI (primer 566/567) into pSG1154 & unpublished \\
\hline pSJ4001 & liaH (4 - end) KpnI/XhoI (primer 560/561) into pSH4 & unpublished \\
\hline pSJ4002 & liaF (4 - end) KpnI/XhoI (primer 564/565) into pSH4 & unpublished \\
\hline pSJ4003 & liaS (4 - end) KpnI/XhoI (primer 566/567) into pSH4 & unpublished \\
\hline pSJ6401 & liaH (4 - end) KpnI/XhoI (primer 560/561) into pSG1164 & unpublished \\
\hline pSK601 & $\mathrm{P}_{\text {wapA }}(-170$ - 128) EcoRI/Bam HI (primer 480/481) into pAC6 & Köcher, unpublished \\
\hline pSK602 & $\mathrm{P}_{y d h E}(-170-128) E c o \mathrm{RI} /$ Bam HI (primer $\left.480 / 481\right)$ into pAC6 & Köcher, unpublished \\
\hline pTM1 & $\mathrm{P}_{\text {liaI }}(-109-72)$ EcoRI/BamHI (primer 099/100) into pAC6 & Jordan et al., 2006 \\
\hline
\end{tabular}

Positions of cloned fragments are given relative to the start codon.

${ }^{a}$ For reasons of clarity, the effects of mutations in liaF and liaS are given at the level of LiaF and LiaS protein.

TM stands for transmembrane region, SD for Shine-Dalgarno sequence

Table 6.4. Primer

\begin{tabular}{lll}
\hline Nr. & Name & Sequence \\
\hline TM001 & yvcB-IDM fwd & GGACAAGCTTGTtTGCCTCAACTATtGCGAGTG \\
TM002 & yvcB-IDM rev & AGCAGGATCCGCAAGTGAATGTCCTAAGGC \\
TM003 & yvkN-IDM fwd & GTAAAAAGCTTACGGCAACCAAGGAGG \\
TM004 & yvkN-IDM rev & AGCAGGATCCGTCCATTAACTGGATGTGCCG \\
TM005 & ywkC-IDM fwd & GGACAAGCTTGGTAGCAAGTGAACTCGGC \\
TM006 & ywkC-IDM rev & AGCAGGATCCCGCTTTCGTCCTGCCTTTGC \\
TM007 & yvrL-check & CAAGCGCACCTTGACTTGG \\
TM008 & yvrIH-check & GCAGATACATTGCAATCAGCG \\
TM009 & yvaZ-check & CAGCGTTGGGATTATCCG \\
TM010 & lacZ-check rev & CTTCGCTATTACGCCAGCTGG \\
TM021 & bceR-up fwd & TCGTAGTAGGATACGACTTCGC \\
TM022 & bceR-up rev (cat3') & GGGTAACTAGCCTCGCCGGTCCACGATACATCATAGGACCATCCCG \\
\hline
\end{tabular}


Supplementary Material

\begin{tabular}{|c|c|c|}
\hline Nr. & Name & Sequence \\
\hline TM023 & bceS-do fwd (cat5') & CTTGATAATAAGGGTAACTATTGCCGAACATGTCATAAGCGTGTGACG \\
\hline TM024 & bceS-do rev & GCGCCACGCTAAAGATGAGCG \\
\hline TM025 & yvcP-up fwd & GATTACCTCGTTGATATCGG \\
\hline TM026 & yvcP-up rev (mls3') & CGATTATGTCTTTTGCGCAGTCGGCTGGTACGGATTTGCCTGCACC \\
\hline TM027 & yvcQ-do fwd (mls5') & GAGGGTTGCCAGAGTTAAAGGATCCGGCCATAAGCTGTATGCGG \\
\hline TM028 & yvcQ-do rev & CCAACACACTCAAGGTATCC \\
\hline TM029 & liaS-up fwd & GCTTTATCAGCAAGCGGTGACG \\
\hline TM030 & liaS-up rev (kan) & СCTATCACCTCAAATGGTTCGCTGTCCCGTTGTCATGCGGATGGC \\
\hline TM031 & liaG-up fwd & TTGTCGTCGGAATCGCATTGGC \\
\hline TM032 & liaG-up rev (kan) & CCTATCACCTCAAATGGTTCGCTGCACATCTTTAACGACGACGGC \\
\hline TM033 & liaG-do fwd (kan) & CGAGCGCCTACGAGGAATTTGTATCGCCAATCGACATCAAAACGGACA \\
\hline TM034 & liaG-do rev & TTACCCGGCGTTTGACTCGC \\
\hline TM035 & liaF fwd (HindIII) & AGGAAGCTTAGAAAGGAGGCGGACACCAGG \\
\hline TM036 & liaF rev (EcoRI) & TCCGAATTCTTTCTCATACGTACTTCACATCC \\
\hline TM037 & pXT-check fwd & CCTTACCGCATTGAAGGCC \\
\hline TM038 & pXT-check rev & GTATTCACGAACGAAAATCGCC \\
\hline TM044 & liaS-fwd (BamHI) & ACGGGATCCCGGTAGTGTGGATGTGAAGTACG \\
\hline TM045 & liaS-rev (BamHI) & ACGGGATCCTCATCAATCAATAATACTCGAATCACG \\
\hline TM046 & liaS-rev (HindIII) & ACGAAGCTTTCATCAATCAATAATACTCGAATCACG \\
\hline TM047 & liaS-do fwd (kan) & CGAGCGCCTACGAGGAATTTGTATCGGGCACTCAAATCGAAGTGAAGG \\
\hline TM048 & liaS-do rev & AACCGGGCTGGGAAACGAGGTC \\
\hline TM051 & liaS-checkfwd & CGATGATGACATCAGCCGTGC \\
\hline TM052 & liaS-checkrev2 & GCTCGACCATCCTGATCCGC \\
\hline TM053 & liaS-checkrev1 & CCGAACCAGCTTGATGACAGC \\
\hline TM054 & liaF-checkfwd & GTAAAACATCCCGACATGCGC \\
\hline TM055 & liaF-checkrev & CGTTCAGGTCAAACGGCTGC \\
\hline TM056 & kan-checkfwd & CATCCGCAACTGTCCATACTCTG \\
\hline TM057 & mls-checkfwd & СCTTAAAACATGCAGGAATTGACG \\
\hline TM058 & spec-checkfwd & GTTATCTTGGAGAGAATATTGAATGGAC \\
\hline TM075 & liaF-fwd(StuI) & ACGAGGCCTAGAAAGGAGGCGGACACCAGG \\
\hline TM076 & liaF-rev(StuI) & CACAGGCCTCTCATACGTACTTCACATCC \\
\hline TM077 & liaS-fwd(StuI) & CACAGGCCTCGGTGATGTGGATGTGAAGTACC \\
\hline TM078 & liaS-rev(StuI) & ACGAGGCCTTCATCAATCAATAATACTCGAATCACG \\
\hline ТМ079 & yvcB-RT fwd & GTGCCGATAATAAGGATTGG \\
\hline TM080 & yvcB-RT rev & GCAAGTGAATGTCCTAAGGC \\
\hline TM081 & ywkC-RT fwd & ACAAATCTCTGAAGGCACGG \\
\hline TM082 & ywkC-RT rev & CCTGCCTTTGCTGTAAAAGG \\
\hline TM083 & yvaZ-RT fwd & TCAGGAAATAAACCGGCTGC \\
\hline TM084 & yvaZ-RT rev & AGAAGCTAGGAAAAGCAATGC \\
\hline TM085 & yvrH-RT fwd & GTTTTGATTTGCCAAGGCTC \\
\hline TM086 & yvrH-RT rev & CCACAATCGCTTTCTCATCG \\
\hline TM087 & yvrI-RT fwd & AAGGAGATAATGAAGCATCCC \\
\hline TM088 & yvrI-RT rev & TTCATATACTTGACGATGCGG \\
\hline TM089 & yvrL-RT fwd & CAATTGGTCTGTTGTATGAGC \\
\hline TM090 & yvrL-RT rev & TTCCTTGTTCTCGGATTATGC \\
\hline TM091 & yvkN-RT fwd & CAGTGTTAAAGCTGATTCAGG \\
\hline TM092 & yvkN-RT rev & GAACAATATGTGTGTTGCTGC \\
\hline TM093 & liaR-RT fwd & ATTGAAGTCATCGGCGAAGC \\
\hline TM094 & liaR-RT rev & AAAGCTCCCGGCAAATTTGC \\
\hline TM095 & 16S-RT fwd & TGAGTAACACGTGGGTAACC \\
\hline
\end{tabular}


Supplementary Material

\begin{tabular}{|c|c|c|}
\hline Nr. & Name & Sequence \\
\hline TM096 & 16S-RT rev & TCACCAACTAGCTAATGCGC \\
\hline TM097 & pIC333-seq1 & GCTGGCTTTAAAGTGCCATGG \\
\hline TM098 & pIC333-seq2 & TATCCGTACTTATGTTATAAGG \\
\hline TM099 & PliaI-fwd (EcoRI) & CCATGAATTCCCGGTGCGAGATACGACTCC \\
\hline TM100 & PliaI-rev (BamHI) & CGATGGATCCTCCTCCAAAAAAGACGGAGATCCC \\
\hline TM101 & liaF-fwdEP & GAAAGGAGGCGGACACCAGG \\
\hline TM102 & liaF-revEP & CTCATACGTACTTCACATCC \\
\hline TM103 & liaF-uprevEP & GCAAAAACATACTGATGCCG \\
\hline TM104 & liaF-dofwdEP & GCGTCAACTGATTTTAGCG \\
\hline TM105 & liaS-fwdEP & GGTGATGTGGATGTGAAGTACG \\
\hline TM106 & liaS-revEP & CCGCGAGCCCCATTCTGACC \\
\hline TM107 & liaS-uprevEP & AAGGAGCAGGCTGATTCCCG \\
\hline TM108 & liaS-dofwdEP & GAAGGTCCCGATTTTTCCGG \\
\hline TM109 & liaR-fwdEP & ATTGATTGATGATCATGAAATGG \\
\hline TM110 & liaR-revEP & GGCGCAAACAAATAGCAGCC \\
\hline TM111 & liaR-uprevEP & GCTGCCGTCCGATGCTTCGC \\
\hline TM112 & liaR-dofwdEP & CAAAGCTGGATGTCAGTGACCG \\
\hline TM125 & liaH-RTfwd & TGAAACAGCACACGATTGCC \\
\hline TM126 & liaH-Rtrev & GTTTGCCTGTTCATAGGAAGC \\
\hline TM127 & liaFpDG148fwd & AAGGAGGAAGCAGGTATGACAAAAAAACAGCTTCTCGGATTGATCATTGC \\
\hline TM128 & liaFpDG148rev & $\overline{\text { GACACGCACGAGGTCTCATACGTACTTCACATCCACATCACCG }}$ \\
\hline TM129 & liaSpDG148fwd & AAGGAGGAAGCAGGTATGAGAAAAAAAATGCTTGCCAGCCTCCAATGGC \\
\hline TM130 & liaSpDG148rev & GACACGCACGAGGTTCATCAATCAATAATACTCGAATCACG \\
\hline TM131 & liaF-fwd (PacI) & TACGTTAATTAAGAAAGGAGGCGGACACCAGG \\
\hline TM132 & liaF-rev (BamHI) & TACGGGATCCCTCATACGTACTTCACATCC \\
\hline TM133 & liaS-fwd (PacI) & TACGTTAATTAACGGTGATGTGGATGTGAAGTACG \\
\hline TM134 & liaS-rev (BamHI) & TACTGGATCCTCATCAATCAATAATACTCGAATCACG \\
\hline TM135 & cat-fwd & CAGCGAACCATTTGAGGTGATAGGCGGCAATAGTTACCCTTATTATCAAG \\
\hline TM136 & cat-rev & CGATACAAATTCCTCGTAGGCGCTCGGCCAGCGTGGACCGGCGAGGCTAGTTACCC \\
\hline TM137 & kan-fwd & CAGCGAACCATTTGAGGTGATAGG \\
\hline TM138 & kan-rev & CGATACAAATTCCTCGTAGGCGCTCGG \\
\hline TM139 & mls-fwd & CAGCGAACCATTTGAGGTGATAGGGATCCTTTAACTCTGGCAACCCTC \\
\hline TM140 & mls-rev & CGATACAAATTCCTCGTAGGCGCTCGGGCCGACTGCGCAAAAGACATAATCG \\
\hline TM141 & spec-fwd & $\begin{array}{l}\overline{\text { CAGCGAACCATTTGAGGTGATAGGGACTGGCTCGCTAATAACGTAACGTGACTGGCA }} \\
\text { AGAG }\end{array}$ \\
\hline TM142 & spec-rev & $\begin{array}{l}\text { CGATACAAATTCCTCGTAGGCGCTCGGCGTAGCGAGGGCAAGGGTTTATTGTTTTCT } \\
\text { AAAATCTG }\end{array}$ \\
\hline TM143 & Tc fwd 1 & CAGCGAACCATTTGAGGTGATAGGTCTTGCAATGGTGCAGGTTGTTCTC \\
\hline TM144 & Tc fwd 2 & CAGCGAACCATTTGAGGTGATAGGGCTTATCAACGTAGTAAGCGTGG \\
\hline TM145 & Tc rev & CGATACAAATTCCTCGTAGGCGCTCGGGAACTCTCTCCCAAAGTTGATCCC \\
\hline TM146 & cat-check rev & $\overline{\text { GTCTGCTTTCTTCATTAGAATCAATCC }}$ \\
\hline TM147 & kan-check rev & CTGCCTCCTCAтсCTCTтCAтCC \\
\hline TM148 & mls-check rev & GTTTTGGTCGTAGAGCACACGG \\
\hline TM149 & spec-check rev & CGTATGTATTCAAATATATCCTCCTCAC \\
\hline TM150 & tc-check rev & CATCGGTCATAAAATCCGTAATGC \\
\hline TM153 & liaF-upfwd2 & TTCGCAGACGATGTGGAAGCGG \\
\hline TM154 & liaF-dorev2 & GCCGATGACTTCAATATCGG \\
\hline TM156 & rpsJ-RTfwd & GAAACGGCAAAACGTTCTGG \\
\hline TM157 & rpsJ-Rtrev & GTGTTGGGTTCACAATGTCG \\
\hline TM158 & rpsE-RTfwd & GCGTCGTATTGACCCAAGC \\
\hline TM159 & rpsE-Rtrev & TACCAGTACCGAATCCTACG \\
\hline
\end{tabular}




\begin{tabular}{|c|c|c|}
\hline Nr. & Name & Sequence \\
\hline TM160 & PliaI-fwd(74) & CGATGAATTCGATACGACTCCGGTCTTA \\
\hline TM161 & PliaI-fwd(70) & CGATGAATTCCGACTCCGGTCTTATATAAA \\
\hline TM162 & PliaI-fwd(66) & CGATGAATTCTCCGGTCTTTATATAAAAATC \\
\hline TM163 & PliaI-fwd(62) & CGATGAATTCGGTCTTTATATAAAAATCAATC \\
\hline TM164 & PliaI-fwd(58) & CGATGAATTCTTATATAAAAATCAATCTCTG \\
\hline TM165 & PyhcY-rev & CGATGGATCCGTGTTGCTTTGATATCGTGCC \\
\hline TM166 & PyhcY-fwd(202) & CGAT GAATTCGACAGTGAAAAGCGACTTGCC \\
\hline TM167 & PyhcY-fwd(99) & CGATGAATTCGTAAATGATCCGGCTTTTTC \\
\hline TM168 & PyhcY-fwd(87) & CGATGAATTCGCTTTTTCTTTTTCTCATCC \\
\hline TM169 & PyhcY-fwd(80) & CGATGAATTCCTTTTTCTCATCCAAAAGTCTG \\
\hline TM170 & PyhcY-fwd(55) & CGATGAATTCGAAAATCATCCTACAAGTG \\
\hline TM171 & PyhcY-fwd(46) & CGATGAATTCCCTACAAGTGAAGCAATGAA \\
\hline TM172 & PyhcY-fwd(29) & CGAT GAATTCGAAATACAAAAAACTGGTATAATC \\
\hline TM173 & cat-checkfwd & CTAATGTCACTAACCTGCCC \\
\hline TM174 & yxjM-upfwd & CCAAGAAAGCAGACCGGATGC \\
\hline TM175 & yxjM-uprev(kan) & CCTATCACCTCAAATGGTTCGCTGGCAATTCAAAATGAGAATAAGGC \\
\hline TM176 & yxjM-dofwd(kan) & CGAGCGCCTACGAGGAATTTGTATCGCCTGAAAATTGAGCTGTCATTGCC \\
\hline TM177 & yxjM-dorev & GCATGTTTCCTAATGTCAATTGGC \\
\hline TM178 & yxjL-upfwd & GCCTTTAGACTCCCTTCACGGC \\
\hline TM179 & yxjL-uprev(kan) & CCTATCACCTCAAATGGTTCGCTGTGATGACGTAGCGGAAGCC \\
\hline TM180 & yxjL-dofwd(kan) & CGAGCGCCTACGAGGAATTTGTATCGCGTTTTTGCCATTCGAAACGG \\
\hline TM181 & yxjL-dorev & CGTTTTGAAGTTCTTCAACGGC \\
\hline TM182 & PliaI-fwdcheck & GGCCAAAGCAGAAAGGTCCG \\
\hline TM183 & pJPM122-catlacZfwd(kan) & CGAGCGCCTACGAGGAATTTGTATCGGCCAGATGCTACACAATTAGGC \\
\hline TM184 & pJPM122-catlacZrev1(kan) & CCTATCACCTCAAATGGTTCGCTGCCCTGATTCTGTGGATAACCG \\
\hline TM185 & pJPM122-catlacZrev2(kan) & CCTATCACCTCAAATGGTTCGCTGCGTGAGTTTTCGTTCCACTGAGCG \\
\hline TM186 & PrpsD-fwd(HindIII) & $\overline{A G G A A G C T T C G A G C A T A T G A T A A T G A A A G G C ~}$ \\
\hline TM187 & PrpsD-rev(BamHI) & AGCCGGATCCCCGTCTGCTCTATTCGACCATGC \\
\hline TM188 & Pveg-fwd(HindIII) & AGGAAGCTTGTCAAAATAATTTTATTGACAACG \\
\hline TM189 & Pveg-rev(BamHI) & AGCCGGATCCTACCTAAATTCCCATCAAGCG \\
\hline TM196 & liaF-up fwd & AAGGATTTGCGGTCAAGTCC \\
\hline TM197 & liaF-up rev (kan) & CCTATCACCTCAAATGGTTCGCTGGCAATGATCAATCCGAGAAGC \\
\hline TM198 & liaF-do fwd (kan) & CGAGCGCCTACGAGGAATTTGTATCGGATGTGGATGTGAAGTACG \\
\hline TM199 & liaF-do rev & TTCAAGCCGTATGAGGAGGC \\
\hline TM200 & liaR-up fwd & GCTGTCATCAAGCTGGTTCGG \\
\hline TM201 & liaR-up rev (kan) & CCTATCACCTCAAATGGTTCGCTGCGATGCTTCGCCGATGACTTC \\
\hline TM202 & liaR-do fwd (kan) & CGAGCGCCTACGAGGAATTTGTATCGACGCACACCGAAATCATCTCG \\
\hline TM203 & liaR-do rev & CTCTTCATCTGATCCGACACAGC \\
\hline TM204 & liaG-fwd (EcoRI) & CCATGAATTCTCCCTTCCGCACGTATCAATTCGC \\
\hline TM205 & liaG-rev (BamHI) & AGCCGGATCCTTTGTCATTCCTGGTG \\
\hline TM206 & liaF-fwd (EcoRI) & CCATGAATTCGAAAGGAGGCGGACACCAGG \\
\hline TM209 & liaR-uprev (HindIII) & ATCGAAGCTTCGATGCTTCGCCGATGACTTC \\
\hline TM210 & liaR-dofwd (PciI) & ATCGACATGTACGCACACCGAAATCATCTCG \\
\hline TM211 & liaF-fwd (XhoI) & AGCTCTCGAGGAAAGGAGGCGGACACCAGG \\
\hline TM212 & liaF1-rev (BamHI) & AGCTGGATCCATATTTTTTAAGGAAATAGCC \\
\hline TM213 & liaF2-rev (BamHI) & AGCTGGATCCTTTCTCATTCGGTTCAAATATCGG \\
\hline TM214 & liaI-fwd (XhoI) & AGCT CTCGAGGGAAAACGAAAGGAGGATCTGC \\
\hline TM215 & liaI-rev (BamHI) & AGCTGGATCCAGATTGGTAGGCAGCAGAAGCC \\
\hline TM216 & liaS-fwd (XhoI) & AGCTCTCGAGCGGTGATGTGGATGTGAAGTACG \\
\hline TM217 & liaS-rev (BamHI) & AGCT GGATCCCATATACCCTGAGGCGAAACCG \\
\hline
\end{tabular}




\begin{tabular}{|c|c|c|}
\hline Nr. & Name & Sequence \\
\hline TM218 & PliaF-157fwd (EcoRI) & CCATGAATTCCAGTTCGCCGTATTCATTTGCTG \\
\hline TM219 & PliaF-250fwd (EcoRI) & CCATGAATTCCGTATCTGTCACGCTGACAAGCG \\
\hline TM220 & PliaF-336fwd (EcoRI) & CCATGAATTCGCAAATCTGGTTCATGTATCAGGC \\
\hline TM221 & PliaF-546fwd (EcoRI) & CCATGAATTCGATCTGGCAGTACGAACCTCAAGCG \\
\hline TM222 & PliaF-824fwd (EcoRI) & CCATGAATTCGCAGGCCTAGGTTCATAAATGGC \\
\hline TM223 & yhcZ-fwd (BsaI) & ACCTGGTCTCAATATGAAAATTGTCATTGCTG \\
\hline TM224 & yhcZ-rev (BamHI) & TCTAGGATCCGTTCATTTTGAGATCTCTCC \\
\hline TM225 & liaH-up fwd & CTTGTTATTCGTCACTGCC \\
\hline TM226 & liaH-up rev (kan) & ССTATCACCTCAAATGGTTCGCTGGTCCTTCATGAACTGACGC \\
\hline TM227 & yhcY-probe fwd & $\overline{\text { GAGTTGCTGAGTCTGACAAACC }}$ \\
\hline TM228 & yhcY-probe rev & TCGTGAAGCTCCTGAGCGAGGC \\
\hline TM229 & yhdA-probe fwd & GCAGAACAAGAATTGCAGCATCC \\
\hline TM230 & yhdA-probe rev & CTGCTTTTGCGAACATGCTGAG \\
\hline TM231 & PliaI wobble1 & ACAT GAATTCGAGAWACSASTCCGKTMTTMTATAAAAATCAATCTCTGATTCG \\
\hline TM232 & PliaI wobble 2 & ACAT GAATTCGAGATACGACTCCGGTCTTATWTWWWWWTCAATCTCTGATTCG \\
\hline TM233 & GFP-checkfwd & CGTAGAGGATCGAGATCTCG \\
\hline TM234 & GFP-checkrev & GGTTATGCTAGTTATTGCTCAGCG \\
\hline TM235 & PhoA-checkfwd & GCATGAAGCAGATCGGTAACG \\
\hline TM236 & PhoA-checkrev & CCATTAAGTCTGGTTGCTAACAGC \\
\hline TM237 & liaF1-rev (KpnI) & AGCTGGTACCGAATATTTTTTAAGGAAATAGCC \\
\hline TM238 & liaF2-rev (KpnI) & AGCTGGTACCTGTTTCTCATTCGGTTCAAATATCGG \\
\hline TM239 & liaI-rev (KpnI) & AGCTGGTACCTGAGATTGGTAGGCAGCAGAAGCC \\
\hline TM240 & liaS-rev (KpnI) & AGCTGGTACCCATATACCCTGAGGCGAAACCG \\
\hline TM241 & PyozJ-rev (BamHI) & AGCCGGATCCCGGAAGTCAATCCAAGTAACAGC \\
\hline TM242 & PyozJ-fwd (EcoRI) & ССАTGAATTCCCATTGTATTCAATAGAAGGC \\
\hline TM243 & PRNA-fwd (EcoRI) & CCATGAATTCGGTATGCTGGTATCTGCTGC \\
\hline TM244 & PRNA-rev (BamHI) & AGCCGGATCCGCAATATAAGATATGGACTCAGCG \\
\hline TM245 & PRNA-rev2 (BamHI) & AGCCGGATCCGGATCAACAATAATGCCCAATGC \\
\hline TM246 & liaS-upfwd (BamHI) & AGCCGGATCCGAAAGGAGGCGGACACCAGG \\
\hline TM247 & liaSclean-uprev & CCATTTCATGATCATCAATCTCTCATACGTACTTCACATCC \\
\hline TM248 & liaSclean-dofwd & CGTATGAGAGATTGATGATCATGAAATGGTCAGAATGG \\
\hline TM249 & liaS-dorev (EcoRI) & CCAT GAATTCAACCGGGCTGGGAAACGAGG \\
\hline TM250 & yhcZ-BsaI & ACCTGGTCTCATCGAGAAAATTGTCATTGCTGATGATC \\
\hline TM251 & yhcZ-up rev (kan) & ССТАTCACCTCAAATGGTTCGCTGCGCAGACCCTTTCTGACAAC \\
\hline TM252 & yhcZ-do fwd (kan) & $\overline{\text { CGAGCGCCTACGAGGAATTTGTATCGGCAAAACTTGAAGTGGCCGATCG }}$ \\
\hline TM253 & pMAD-checkrev & $\overline{\text { GTTACGTTACACATTAACTAGAC }}$ \\
\hline TM254 & pMAD-checkfwd & CTGATGGTCGTCATCTACCTGCC \\
\hline TM255 & yhcY-probe fwd1 & CTAATACGACTCACTATAGGGAGAGAGTTGCTGAGTCTGACAAACC \\
\hline TM256 & yhdA-probe fwd1 & CTAATACGACTCACTATAGGGAGAGCAGAACAAGAATTGCAGCATCC \\
\hline TM257 & yhxB-fwd BsaI & ACCTGGTCTCGAATTCGCTTTTACACGATGCGGTCG \\
\hline TM258 & liaF-probe Tn7fwd & CTAATACGACTCACTATAGGGAGACGTGATTGGCTTGGCTCCG \\
\hline TM259 & PyhcY(75)-fwd & CGATGAATTCTCTCATCCAAAAGTCTGAAAG \\
\hline TM260 & PyhcY(65)-fwd & CGATGAATTCAAGTCTGAAAGAAAATCATCCTACAAGTG \\
\hline TM261 & yhcY-fwd (BsaI) & ACCTGGTCTCATCGAGGAAAAATTAAAAACGCTGAAAACG \\
\hline TM262 & yhcY-rev (BamHI) & TCTAGGATCCTCATACCGCCCCTCCTTTTCG \\
\hline TM263 & PyhdA-rev (BamHI) & CGATGGATCCCGTGTGATACAGAGCTGCAATATAGG \\
\hline TM264 & PyhdA-fwd (EcoRI) & CGATGAATTCCCAGGATGATATTGAAGTCGTCG \\
\hline TM265 & PliaI wobble3 & ACATGAATTCGAGAaACaAaTCCGaTaTTATATAAAAATCAATCTCTGATTCG \\
\hline TM266 & PliaI wobble4 & ACATGAATTCGAGATACGACTCCGGTCTTCTATACAAATCAATCTCTGATTCG \\
\hline TM267 & PliaI wobble5 & ACATGAATTCGAGAaACaAaTCCGGTCTTATATAAAAATCAATCTCTGATTCG \\
\hline TM268 & PliaI wobble6 & ACATGAATTCGAGATACGACTCCGaaaTTATATAAAAATCAATCTCTGATTCG \\
\hline TM269 & PaprE-fwd (EcoRI) & CGATGAATTCCTTATTTCTTCCTCCCTCTC \\
\hline TM270 & PaprE-rev (BamHI) & CGATGGATCCCGCAAACAACAAGCTGATCCAC \\
\hline TM271 & aprE-fwd (HindIII) & CGATAAGCTTAAAGGAGAGGGTAAAGAGTGAG \\
\hline
\end{tabular}




\begin{tabular}{|c|c|c|}
\hline Nr. & Name & Sequence \\
\hline TM272 & aprE-rev (BamHI) & CGATGGATCCGAGTCAATTCCGCTGTCG \\
\hline TM273 & aprE-up fwd & GTTGACATTCGGCACACTCC \\
\hline TM274 & aprE-up rev & СCTATCACCTCAAATGGTTCGCTGGACATGTTGCTGAACGCCATCG \\
\hline TM275 & aprE-do fwd & $\overline{\text { CGAGCGCCTACGAGGAATTTGTATCGAAACGCGCAAGTCCGTGATCG }}$ \\
\hline TM276 & aprE-do rev & CATTTCCACACAGACAACGG \\
\hline TM277 & salA-up fwd & AAGATTGGTGGACAGCAGG \\
\hline TM278 & salA-up rev & CCTATCACCTCAAATGGTTCGCTGGGTTCGCGCATTTCTCCG \\
\hline TM279 & salA-do fwd & CGAGCGCCTACGAGGAATTTGTATCGTGACGAAAATCATCCAATCGG \\
\hline TM280 & salA-do rev & TATCTCAAGCGCAAACCGATG \\
\hline TM281 & abrB-up fwd & TATCAACGAGCTGAGTTTCCG \\
\hline TM282 & abrB-up rev & CCTATCACCTCAAATGGTTCGCTGCAACTTTACGTACAATACCAGTAG \\
\hline TM283 & abrB-do fwd & 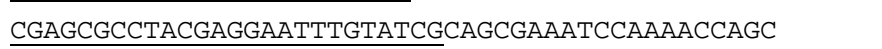 \\
\hline TM284 & abrB-do rev & TTCTTTACTTGGTCCCAACCC \\
\hline TM285 & scoC-up fwd & AACCTCTTCCGCTTCCGG \\
\hline TM286 & scoC-up rev & CCTATCACCTCAAATGGTTCGCTGGAGCCTTGCTAAGCTGAGCC \\
\hline TM287 & scoC-do fwd & $\overline{\text { CGAGCGCCTACGAGGAATTTGTATCGGATGAACCGGCTGAAGAGC }}$ \\
\hline TM288 & scoC-do rev & ACGTTTCCATGTGCGCATGC \\
\hline TM289 & sinR-up fwd & GCCAAAAGACCTAGATGGTG \\
\hline TM290 & sinR-up rev & ССTATCACCTCAAATGGTTCGCTGATGTCATCACCTTCCTTGTG \\
\hline TM291 & $\operatorname{sinR}$-do fwd & $\overline{\text { CGAGCGCCTACGAGGAATTTGTATCGGATGACATCCGGGGTATCG }}$ \\
\hline TM292 & sinR-do rev & TAGGAGTTGCTTCTGCAGC \\
\hline TM293 & yhdA-probe rev T7 & CTAATACGACTCACTATAGGGAGACTGCTTTTGCGAACATGCTGAG \\
\hline TM294 & yhcY-probe rev T7 & CTAATACGACTCACTATAGGGAGATCGTGAAGCTCCTGAGCGAGGC \\
\hline TM295 & PliaF-rev1 (BamHI) & AGCCGGATCCGCCATTTATGAAACCTAGGC \\
\hline TM296 & PliaF-rev2 (BamHI) & AGCCGGATCCCATTCGGTTTCATCCTTCTCATTC \\
\hline TM297 & liaG-fwd (XhoI) & AGCTCTCGAGGAATGAATGAGAAGGATGAAACCG \\
\hline TM298 & liaG-rev2 (BamHI) & AGCTGGATCCGGACATCCTTGCTATCCG \\
\hline TM299 & liaS-rev2 (BamHI) & AGCCGGATCCAAGCGGATCGAGCCGATAG \\
\hline TM300 & PliaI-423 (EcoRI) & GATCGAATTCGCAGTCAGTGCTGTTAATGTTCC \\
\hline TM301 & degU-up fwd & AAGCCCATAAGCTGCAGG \\
\hline TM302 & $\operatorname{deg} U$-upfwd2 & CAGAGGATCAGAGGCTAGC \\
\hline TM303 & degU-up rev & СCTATCACCTCAAATGGTTCGCTGTATCCGTTTAACACCTTCACG \\
\hline TM304 & degU-do fwd & CGAGCGCCTACGAGGAATTTGTATCGTAAACGACCGGACGCAAGCC \\
\hline TM305 & degU-do rev & CAAATGAGTGCCGATTACCGC \\
\hline TM306 & $\operatorname{deg} \mathrm{U}$-do rev2 & AAGCAGCTGATCTCTGAGTC \\
\hline TM307 & spo0A-up fwd & TATCAGAGATTCTGCTGCTGGC \\
\hline TM308 & spo0A-up rev & ССTATCACCTCAAATGGTTCGCTGAGCGACAGGCATTCCTGTCC \\
\hline TM309 & spo0A-do fwd & CGAGCGCCTACGAGGAATTTGTATCGGTTGCGGATAAGCTGAGG \\
\hline TM310 & spo0A-do rev & GGAAGAACCTGAGACACCG \\
\hline TM322 & ackA-upfwd & GGAACTGACCATTCTTGATCCAGC \\
\hline TM323 & ackA-uprev & CCTATCACCTCAAATGGTTCGCTGCCATTTAAACATTGTCATGTCGG \\
\hline TM324 & ackA-dofwd & CGAGCGCCTACGAGGAATTTGTATCGCGACTGATGAAGAAGTCATGATTGCG \\
\hline TM325 & ackA-dorev & CGACGGAAGTATCAAGACCTCC \\
\hline TM326 & pta-upfwd & GCTCTACCACTGATACGTAGG \\
\hline TM327 & pta-uprev & СCTATCACCTCAAATGGTTCGCTGGCGTTCTACGAATGCTTGTACAAGG \\
\hline TM328 & pta-dofwd & CGAGCGCCTACGAGGAATTTGTATCGCGCTGAAGATGTTTACAATCTCGC \\
\hline TM329 & pta-dorev & CGCTTCCTTTACACCTTGATTGC \\
\hline TM330 & liaG-rev3 (BamHI) & AGCTGGATCCCCGGACATCCTTGCTATCCG \\
\hline TM366 & PyuzG-fwd (EcoRI) & CCATGAATTCCAGGAATTAGCTGAATATCTTCG \\
\hline TM367 & PyuzG-rev (BamHI) & CGATGGATCCTGCGACTACCCAGATCATTGCC \\
\hline TM368 & PguaC-fwd (EcoRI) & CCATGAATTCTGCGACTACCCAGATCATTGCC \\
\hline TM369 & PguaC-rev (BamHI) & CGATGGATCCCAGGAATTAGCTGAATATCTTCG \\
\hline TM370 & liaF-fwdSD (XhoI) & AGCTCTCGAGAATGACAAAAAAACAGCTTCTCG \\
\hline TM371 & liaF-rev3 (BamHI) & CGATGGATCCGTTTTTCAAAAATAGAAACGCGG \\
\hline
\end{tabular}




\begin{tabular}{|c|c|c|}
\hline Nr. & Name & Sequence \\
\hline TM405 & liaG-fwdSD (XhoI) & AGCTCTCGAGAATGAAAAAAATGCTTGGC \\
\hline TM406 & liaS-fwdSD (XhoI) & AGCTCTCGAGTATGAGAAAAAAAATGCTTGCC \\
\hline Tm407 & liaI-fwdSD (XhoI) & AGCTCTCGAGCATGAAAATAAACAAGAAAACAATAGGC \\
\hline TM408 & liaF4-rev (BamHI) & AGCTGGATCCCGGCCAAAACAGCAGATCG \\
\hline TM409 & liaI2-rev (BamHI) & AGCTGGATCCTGCGTATGTCATCAAGC \\
\hline TM410 & pXT-fwd & GTTTAAACAACAAACTAATAGGTGATG \\
\hline TM411 & pXT-rev & GTTATAGTTATTATAACATGTATTCACG \\
\hline TM448 & ptb-upfwd & GGCTATGAGGATGGTGCG \\
\hline TM449 & ptb-uprev & CCTATCACCTCAAATGGTTCGCTGGTGTATCGACGCTTTGCC \\
\hline TM450 & ptb-uprevi & CGAGCGCCTACGAGGAATTTGTATCGGTGTATCGACGCTTTGCC \\
\hline TM451 & ptb-dofwd & 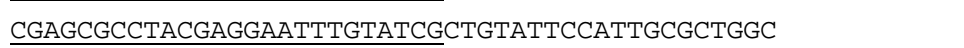 \\
\hline TM452 & ptb-dofwdi & 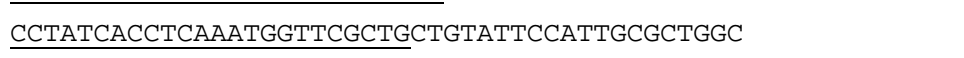 \\
\hline TM453 & ptb-dorev & $\overline{\text { CGCACCTGCGATCACTTTCGC }}$ \\
\hline TM454 & liaS-fwd (BamHI) & ACGGGATCCCGGTGATGTGGATGTGAAGTACG \\
\hline TM455 & liaFclean-dofwd & CGGTGATGTGGATGTGAAGTACG \\
\hline TM456 & liaFclean-uprev & CGTACTTCACATCCACATCACCGTCCTGGTGTCCGCCTCCTTTC \\
\hline TM457 & liaF-upfwd (BamHI) & 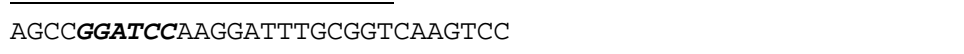 \\
\hline TM458 & liaF-dorev (NcoI) & AGCTCCATGGTTCAAGCCGTATGAGGAGGC \\
\hline TM459 & liaFSclean-uprev & CCATTTCATGATCATCAATCTCCTGGTGTCCGCCTCCTTTC \\
\hline TM460 & liaFSclean-dofwd & $\overline{\text { GATTGATGATCATGAAATGGTCAGAATGG }}$ \\
\hline TM461 & liaSclean-dofwd2 & CCGGAAGAAAAAGGAGAGAACGAACG \\
\hline TM462 & liaSclean-uprev2 & CGTTCGTTCTCTCCTTTTTCTTCCGGCTCATACGTACTTCACATCC \\
\hline TM463 & liaFSclean-uprev2 & CGTTCGTTCTCTCCTTTTTCTTCCGGTCCTGGTGTCCGCCTCCTTTC \\
\hline TM480 & PydhE-rev(BamHI) & CGATGGATCCATCCCGGTGAAGATGGACCG \\
\hline TM481 & PydhE-fwd(EcoRI) & CGATGAATTCTTGATTAAGTCCAGAGCAGCAG \\
\hline TM482 & PwapA-rev(BamHI) & CGATGGATCCTTGCTAGTACATCGGCTGGC \\
\hline TM483 & PwapA-fwd(EcoRI) & CGATGAATTCGCGAATGTGACAGCTGAGGG \\
\hline TM488 & liaFclean-uprev2 & TTTTTTCTCATACGTACTTCACATCCACATCACCGTCCTGGTGTCCGCCTCCTTTC \\
\hline TM489 & liaF-TM1-4fwd (HindIII) & $\begin{array}{l}\text { AGGAAGCTTAGAAAGGAGGCGGACACCAGGAATGCCGATATTTGAACCGAATGAGAA } \\
\text { ACAGGTC }\end{array}$ \\
\hline TM490 & liaF-TM2u3-uprev & AATTAAAGGTGATGCTGAAGAGCCAAAACAGCAGATCGCCTATTCCG \\
\hline TM491 & liaF-TM2u3-dofwd & $\overline{\text { CTCTTCAGCATCACCTTTAATTTATTCGG }}$ \\
\hline TM492 & liaF-195 (EcoRI) & TCCGAATTCTCATCCTATAAAAACAGCCGAGC \\
\hline TM493 & liaF-220 (EcoRI) & TCCGAATTCTCACTCGCTAAAATCAGTTGACGC \\
\hline TM494 & liaF-G234A rev (EcoRI) & TCCGAATTCTTTCTCATACGTACTTCACATCCACATCAGCGATAAATAAGG \\
\hline TM495 & liaS-SD opt.(BamHI) & ACGGGATCCTAAGGAGGAGTACGTATGAGAAAAAAAATGC \\
\hline TM500 & liaF-G128A & GCTTTTTTATCGCTGAGCTGCAAATGATG \\
\hline TM501 & liaF-G149A & CTCTGGTTtTATCGCTGATATCAAAATCG \\
\hline TM502 & liaF-G174A & CGGAGTCATTGCTAACGTTGATATTTATG \\
\hline TM503 & liaF-G195A & CTGTtTtтATAgCAGACATtAATCTGATCG \\
\hline TM504 & liaF-D141A & GTTTGACCTGAACGCTTTAAATGTCTCTGG \\
\hline TM505 & liaF-D154A & GATATCAAAATCGGCTTATCTAAAGCGATG \\
\hline TM506 & liaF-D183A & ATGTACCATCGGCCCTTGAAGTGGC \\
\hline TM507 & liaF-P181A & GATATTTATGTAGCATCGGACCTTGAAG \\
\hline TM508 & liaR-D54A & CAтTTTAATGGCCCTTGTCATGGAGGG \\
\hline TM525 & PydhE-fwd2 (EcoRI) & CGATGAATTCACGATTAGTAGTAGTCTGATCAAAC \\
\hline TM526 & PydhE-fwd3 (EcoRI) & CGATGAATTCGTCAGAATACGATTTGAGAGGG \\
\hline TM529 & liaS cyt.-fwd(BamHI) & ACGGGATCCCGGTGATGTGGATGTGAAGTACGTATGGGCAACCGGTTGAAGACAAGG \\
\hline TM530 & liaS cyt.SD-fwd(BamHI) & ACGGGATCCTAAGGAGGAGTACGTATGGGCAACCGGTTGAAGACAAGG \\
\hline TM531 & liaS C-term.-fwd(HindIII) & AGGAAGCTTTGAAAACGGCAATTTCGCTTATCGG \\
\hline TM532 & liaS C-term.-rev(BsaI) & ACCTGGTCTCAAATTCTCATCAATCAATAATACTCGAATCACG \\
\hline TM533 & liaS N-term.-rev(HindIII) & AGGAAGCTTAAAATGGATTCAATTAATGTATCAATCCT \\
\hline TM534 & liaS 104-106A dofwd & CGCTCGGTGCTGCTGCTATCGGCCTGGCTGCTGATCAGC \\
\hline TM535 & liaS 104-106del dofwd & CGCTCGGTATCGGCCTGGCTGCTGATCAGC \\
\hline TM536 & liaS 104-106A uprev & CCAGGCCGATAGCAGCAGCACCGAGCGGCGGTATCCG \\
\hline
\end{tabular}




\begin{tabular}{|c|c|c|}
\hline Nr. & Name & Sequence \\
\hline TM537 & liaS 104-106del uprev & CCAGGCCGATACCGAGCGGCGGTATCCG \\
\hline TM538 & liaS 202-207A dofwd & GCGAAGCCGCAGCTGCGGCGGCGGCGCTGCTGCTCCATTTACGG \\
\hline TM539 & liaS 202-207del dofwd & GCGAAGCCCTGCTGCTCCATTTACGG \\
\hline TM540 & liaS 202-207A uprev & GCAGCAGCGCCGCCGCCGCAGCTGCGGCTTCGCCTGCCATATGC \\
\hline TM541 & liaS 202-207del uprev & GCAGCAGGGCTTCGCCTGCCATATGC \\
\hline TM542 & liaS 211-214A dofwd & CGCTGCTGCTCGCTGCAGCGGCTGTTACCCTTGAAGGAAA \\
\hline TM543 & liaS 211-214del dofwd & CGCTGCTGCTCGTTACCCTTGAAGGAAA \\
\hline TM544 & liaS 211-214A uprev & GGGTAACAGCCGCTGCAGCGAGCAGCAGCGCCCTCATCTC \\
\hline TM545 & liaS 211-214del uprev & GGGTAACGAGCAGCAGCGCCCTCATCTC \\
\hline TM550 & lacZ-upfwd (BamHI) & CCGCGGATCCCCAGCTTGTTG \\
\hline TM551 & lacZ-uprev & CGGCGCTCAGTTGGAACTCCGCCGATACTGACGGGCTCC \\
\hline TM552 & lacZ-dofwd & ATCGGCGGAGTTCCAACTGAGCGCCGGTCGCTACCATTACC \\
\hline TM553 & lacZ-dorev (BlpI) & GTACGCTCAGCAATGATGACCTCGTTTCCACCGG \\
\hline TM556 & LiaFD237A (EcoRI) & TCCGAATTCTTTCTCATACGTACTTCACAGCCACATCACCG \\
\hline TM557 & LiaFD235A (EcoRI) & TCCGAATTCTTTCTCATACGTACTTCACATCCACAGCACCGATAAATAAGG \\
\hline TM558 & liaI-GFP fwd (KpnI) & GACT GGTACCAAAATAAACAAGAAAACAATAGGC \\
\hline TM559 & liaI-GFP rev (XhoI) & GACTCTCGAGTTTTTTCTTCAAAAATTCTTCCCATTCG \\
\hline TM560 & liaH-GFP fwd (KpnI) & GACT GGTACCGTATTAAAAAGAATCAGAGACATGTTTG \\
\hline TM561 & liaH-GFP rev (XhoI) & GACTCTCGAGTTCATTTGCCGCTTTTGTCTGG \\
\hline TM562 & liaG-GFP fwd (KpnI) & GACT GGTACCAAAAAAATGCTTGGCAAACTGTTGATCAC \\
\hline TM563 & liaG-GFP rev (XhoI) & GACTCTCGAGCCGTATCGCTAGATCCCCGCTG \\
\hline TM564 & liaF-GFP fwd (KpnI) & GACT GGTACCACAAAAAAACAGCTTCTCGG \\
\hline TM565 & liaF-GFP rev (XhoI) & GACTCTCGAGTACGTACTTCACATCCACATCACC \\
\hline TM566 & liaS-GFP fwd (KpnI) & GACT GGTACCAGAAAAAAAATGCTTGCCAGCC \\
\hline TM567 & liaS-GFP rev (XhoI) & GACTCTCGAGATCAATAATACTCGAATCACGTTCG \\
\hline TM568 & LiaF-TM YxjM-upfwd (HindIII) & $\begin{array}{l}\text { AGGAAGCTTAGAAAGGAGGCGGACACCAGGAATGATTTTGTTAGCATTTGTATTTGC } \\
\text { G }\end{array}$ \\
\hline TM569 & LiaF-TM YxjM-uprev & TTTCTCATTCGGTTCAAATATCGGGGAGAAGAGGACGTACAGACC \\
\hline TM570 & LiaF-TM YxjM-dofwd & $\overline{\text { CCGATATTTGAACCGAATGAGAAAC }}$ \\
\hline TM571 & LiaS-TM YxdK-upfwd (BamHI) & $\begin{array}{l}\text { ACGGGATCCCGGTGATGTGGATGTGAAGTACGTATGAAGCTGTTTCTCCGGTCTCAT } \\
\text { GC }\end{array}$ \\
\hline TM572 & LiaS-TM YxdK-uprev & СCTTGTCTTCAACCGGTTGCCATAGGCAAGATAGCCCGCCA \\
\hline TM573 & LiaS-TM YxdK-dofwd & GGCAACCGGTTGAAGACAAGG \\
\hline TM574 & LiaFclean-uprev (XhoI) & GACTCTCGAGTCCTGGTGTCCGCCTCCTTTC \\
\hline TM575 & LiaFclean-dofwd (XhoI) & GACTCTCGAGCGGTGATGTGGATGTGAAGTACG \\
\hline TM577 & PliaG-fwdnat(SmaI) & CCATCCCGGGTCCCTTCCGCACGTATCAATTCGC \\
\hline TM578 & PliaG-fwdopt-10(SmaI) & $\begin{array}{l}\text { CCATCCCGGGTCCCTTCCGCACGTATCAATTCGCAAGCTTTTCTGTTATAATAGAAT } \\
\text { G }\end{array}$ \\
\hline TM579 & PliaG-fwdopt(SmaI) & $\begin{array}{l}\text { CCATCCCGGGTCCCTTCCGCACTTGACAATTCGCAAGCTTTTCTGTTATAATAGAAT } \\
\text { G }\end{array}$ \\
\hline TM580 & liaFexpr (BamHI) & AGCCGGATCCATTCCTGGTGTCCGCCTCC \\
\hline TM581 & liaSexpr (BamHI) & AGCCGGATCCATACGTACTTCACATCCACATC \\
\hline TM582 & liaRexpr (BamHI) & AGCCGGATCCACGTTCGTTCTCTCCTTTTTCTTCC \\
\hline TM627 & PliaI-rev (PstI) & AGTCCTGCAGCCTCCTTTCGTTTTCCTTGTC \\
\hline TM722 & liaR-YFP fwd (KpnI) & ATGCGGTACCGTGATTCGAGTATTATTGATTGATG \\
\hline TM723 & liaR-YFP rev (XhoI) & ATGCCTCGAGATTCACGAGATGATTTCGGTGTGC \\
\hline TM724 & GFP-liaI fwd (SalI) & ATGCGTCGACATGAAAATAAACAAGAAAACAATAGGC \\
\hline TM725 & GFP-liaI rev (EcoRV) & ATGCGATATCTTTTTTCTTCAAAAATTCTTCCC \\
\hline TM726 & GFP-liaH fwd (SalI) & ATGCGTCGACATGGTATTAAAAAGAATCAGAGACATG \\
\hline TM727 & GFP-liaH rev (EcoRV) & ATGCGATATCTTCATTTGCCGCTTTTGTCTGG \\
\hline TM728 & GFP-liaF fwd (SalI) & ATGCGTCGACATGACAAAAAAACAGCTTCTCGG \\
\hline TM729 & GFP-liaF rev (HindIII) & ATGCAAGCTTTACGTACTTCACATCCACATC \\
\hline TM730 & GFP-liaS fwd (SalI) & ATGCGTCGACATGAGAAAAAAAATGCTTGCCAGC \\
\hline TM731 & GFP-liaS rev (HindIII) & ATGCAAGCTTATCAATAATACTCGAATCACGTTCG \\
\hline TM732 & GFP-liaR fwd (SalI) & ATGCGTCGACGTGATTCGAGTATTATTGATTGATG \\
\hline TM733 & GFP-liaR rev (HindIII) & ATGCAAGCTTATTCACGAGATGATTTCGGTG \\
\hline
\end{tabular}




\begin{tabular}{|c|c|c|}
\hline Nr. & Name & Sequence \\
\hline TM734 & PliaG-YFP fwd (KpnI) & ATGCGGTACCGAAGTATTTAGAAGGGAAGGC \\
\hline TM735 & PliaG-YFP rev (XhoI) & ATGCCTCGAGCATTCGGTTTCATCCTTCTC \\
\hline TM736 & PliaI-CFP fwd (KpnI) & ATGCGGTACCCAGTCAGTGCTGTTAATGTTCC \\
\hline TM737 & PliaI-CFP rev (EcoRI) & ATGCGAATTCCATGCAGATCCTCCTTTCGTTTTC \\
\hline TM738 & GFP-liaI fwd (XhoI) & ATGCCTCGAGATGAAAATAAACAAGAAAACAATAGGC \\
\hline TM739 & GFP-liaH fwd (XhoI) & ATGCCTCGAGATGGTATTAAAAAGAATCAGAGACATG \\
\hline TM740 & GFP-liaF fwd (XhoI) & ATGCCTCGAGATGACAAAAAAACAGCTTCTCGG \\
\hline TM741 & GFP-liaS fwd (XhoI) & ATGCCTCGAGATGAGAAAAAAAATGCTTGCCAGC \\
\hline TM742 & GFP-liaR fwd (XhoI) & ATGCCTCGAGGTGATTCGAGTATTATTGATTGATG \\
\hline TM743 & GFP-liaI rev (ClaI) & ATGCATCGATTTTTTTCTTCAAAAATTCTTCCC \\
\hline TM744 & GFP-liaH rev (ClaI) & ATGCATCGATTTCATTTGCCGCTTTTGTCTGG \\
\hline
\end{tabular}

The (universal) linker sequences used for joining reactions are underlined. Restriction sites for cloning are highlighted in bold italics. Nucleotides given in small letters represent mismatches. Sequences underlined are inverse and complementary to other fragments for PCR fusion. 


\section{Curriculum vitae}

\section{Personal Information}

Date of birth:

$25^{\text {th }}$ June 1978

Place of birth: $\quad$ Essen, Germany

Nationality: German

\section{School Education}

1984

1984-1988

1988-1997

Grundschule Nienburg-Weser

Sieburgschule Bad Karlshafen

07/1997

Städtisches Gymnasium Beverungen

Abitur

\section{Scientific Education}

1997-2004

study of biology, Eberhardt-Karls-University Tübingen

2003-2004

diploma thesis

Dept. of Microbiology/Biotechnology (Prof. Dr. Wolfgang

Wohlleben), Eberhardt-Karls-University Tübingen

thesis title:

"Entwicklung eines positiven Selektionssystems für

Streptomyceten"

08/2004 - 11/2007 PhD thesis

Dept. of General Microbiology (Prof. Dr. Jörg Stülke),

Georg-August-University Göttingen

thesis title: $\quad$ "Intramembrane signal transduction and cell envelope stress response in Bacillus subtilis"

04/2007 - 06/2007 Visiting scientist in the group of Dr. Leendert Hamoen, Institute for Cell and Molecular Biosciences, University of Newcastle 